\title{
LINE CONGRUENCE AND TRANSFORMATION OF PROJECTIVE SURFACES
}

\author{
Takeshi SASAKI \\ (Received 17 May 2005)
}

\begin{abstract}
The aim of this article is to present and reformulate systematically what is known about surfaces in the projective 3-space, in view of transformations of surfaces, and to complement this with some new results. Special emphasis will be laid on line congruences and Laplace transformations. A line congruence can be regarded as a transformation connecting one focal surface with the other focal surface. A Laplace transformation is regarded as a method of constructing a new surface from a given surface by relying on the asymptotic system the surface is endowed with. A principal object in this article is a class of projectively minimal surfaces. We clarify the procedure of getting new projectively minimal surfaces from a given one, which was found by F. Marcus, as well as the procedure of Demoulin transformation of projective surfaces.
\end{abstract}

\section{Contents}

$\begin{array}{lll}\text { 0 Introduction } & 104\end{array}$

1 Ruled surfaces $\quad \mathbf{1 0 6}$

1.1 Space curves . . . . . . . . . . . . . . . . . 106

1.2 Ruled surfaces . . . . . . . . . . . . . . . . . . 109

1.3 Fundamental invariants of a ruled surface . . . . . . . . . . . . 111

1.4 Differential invariants of a ruled surface . . . . . . . . . . . . 113

2000 Mathematics Subject Classification: Primary 53A20;

Secondary 53A25, 53A15, 53B10, 53C10, 58A 15.

Keywords and Phrases: projective surface; line congruence; Laplace transformation; projectively minimal surface; affine sphere; Demoulin transformation.

This article is an invited contribution to a special issue of the Kyushu Journal of Mathematics commemorating the sixtieth volume. 
2 Projective theory of surfaces $\quad 114$

2.1 Projective invariants of hypersurfaces . . . . . . . . . . . . . . . 114

2.2 Projective invariants of surfaces . . . . . . . . . . . . . . . . 119

2.3 The Wilczynski frame of a surface . . . . . . . . . . . . . . . . 121

2.4 Demoulin frames of a surface . . . . . . . . . . . . . . . . . 123

2.5 Remarks on higher-dimensional hypersurfaces . . . . . . . . . . . . 127

2.6 Projectively applicable surfaces . . . . . . . . . . . . . . . . . . 128

2.7 Projectively minimal surfaces . . . . . . . . . . . . . . . . . . 134

3 Line congruences I

3.1 Line congruences . . . . . . . . . . . . . . . . . . . . . . . . . 137

$3.2 W$-congruences . . . . . . . . . . . . . . . 138

4 The Laplace transformation $\quad \mathbf{1 4 0}$

4.1 Laplace invariants . . . . . . . . . . . . . . . . . . . . . . . . 140

4.2 The Laplace transformation . . . . . . . . . . . . . . . . . . . . . . 142

4.3 Recursive relations of Laplace invariants . . . . . . . . . . . . . . . . 144

4.4 Periodic Laplace sequences . . . . . . . . . . . . . . . . . . . . 145

4.5 Terminating Laplace sequences . . . . . . . . . . . . . . . . . . . . 148

4.6 The Euler-Poisson-Darboux equation . . . . . . . . . . . . . 150

4.7 The Échell of hypergeometric functions . . . . . . . . . . . . . . . . 154

4.8 Godeaux sequences . . . . . . . . . . . . . . 156

5 Affine spheres and the Laplace transformation 161

5.1 Affine surfaces . . . . . . . . . . . . . . . 161

5.2 Affine spheres . . . . . . . . . . . . . . 163

5.3 Affine polar surfaces . . . . . . . . . . . . . . . . . . 164

5.4 From an affine surface to a projective surface . . . . . . . . . . 165

5.5 Laplace transforms of affine spheres . . . . . . . . . . . . . . . 166

5.6 Tzitzeica transforms of affine spheres . . . . . . . . . . . 168

6 Line congruences II $\quad 169$

6.1 The Weingarten invariant $W \ldots \ldots$. . . . . . . . . . 170

6.2 Covariance of frames . . . . . . . . . . . . . . . . . . 172

6.3 Systems of differential equations of a line congruence . . . . . . . . . 174

6.4 The dual of a line congruence . . . . . . . . . . . . . . . . . . 177 
7 Linear complexes $\quad 178$

7.1 Linear complexes . . . . . . . . . . . . . . . . . . . . . 178

7.2 The Plücker image of a line congruence _ . . . . . . . . . 178

7.3 Line congruences belonging to a linear complex . . . . . . . . . 181

8 Laplace transforms of a line congruence 183

8.1 Laplace transforms of a line congruence . . . . . . . . . . . . . 184

8.2 Laplace transforms of a linear complex . . . . . . . . . . . . 185

9 Invariants of focal surfaces 187

9.1 Invariants of focal surfaces . . . . . . . . . . . . . . . . . . 189

9.2 Line congruences both of whose focal surfaces are quadrics . . . . . 192

10 Construction of $W$-congruences $\quad 194$

10.1 A description of $W$-congruences . . . . . . . . . . . . . . 195

$10.2 W$-congruences whose focal surfaces are quadrics . . . . . . . . 197

10.3 Composition of $W$-congruences _ . . . . . . . . . . . . . . . 199

11 Lie quadrics and Demoulin transforms 201

11.1 Osculating quadrics . . . . . . . . . . . . . . . 202

11.2 Lie quadrics . . . . . . . . . . . . . . . . . . 202

11.3 Demoulin transforms . . . . . . . . . . . . . . . . . 203

11.4 Demoulin lines . . . . . . . . . . . . . . . . . . . . . 206

11.5 Demoulin congruences . . . . . . . . . . . . . . . . . . 207

11.6 An explicit form of Demoulin frames . . . . . . . . . . . . . . 209

12 An intrinsic description of Demoulin transforms of projectively minimal surfaces

12.1 The normalized frame of a Demoulin transform . . . . . . . . . 211

12.2 Second Demoulin transforms . . . . . . . . . . . . . . . . 217

12.3 Coincidence surfaces . . . . . . . . . . . . . . . . 222

13 Transformations of projectively minimal surfaces $\quad 224$

$13.1 W$-congruences of projectively minimal surfaces . . . . . . . 224

13.2 Transformations of projectively minimal surfaces . . . . . . . 226

Appendix A. Line congruences derived from Appell's system $\left(F_{2}\right) \quad 229$

Appendix B. Line congruences in $\mathrm{E}^{3}$ 
References

\section{Introduction}

Transformations of surfaces have been a subject of strong interest to mathematicians for many years, from a geometric point of view as well as from an analytic point of view. Darboux [D1] opened his four-volume book 'Leçons sur la théorie générale des surfaces', with a preface saying that his aim was to investigate new applications to geometry of the theory of partial differential equations. In his book, we can find several ways of extracting geometric conclusions from structures lying in systems of differential equations, which still stimulate us. Even within the last ten years classical transformations such as the Darboux transformation and Bäcklund transformation are still being found to be fundamental to knowing the structure of linear and/or nonlinear differential equations associated with geometric objects, and to constructing surfaces with special properties; this has lead to a renewal of interest in the theory of surfaces.

The aim of this article is to present and reformulate systematically what is known about surfaces in the projective 3-space, in view of transformations of surfaces, and to complement this with some new results. Special emphasis will be laid on line congruences and Laplace transformations. A line congruence can be regarded as a transformation connecting one focal surface with the other focal surface. A Laplace transformation is regarded as a method of constructing a new surface from a given surface by relying on the asymptotic system the surface is endowed with.

A principal object in this article is a class of projectively minimal surfaces. By definition, a surface is projectively minimal if and only if the area functional, constructed in a projectively invariant way, attains an extremal value for this surface. The class of such surfaces includes the class of affine spheres that are fundamental in affine differential geometry and the class of Demoulin surfaces that are characterized by degeneracy of the envelope of Lie quadrics. In the last section of this article, we show that the class of projectively minimal surfaces fits nicely into the framework of line congruences and Laplace transformations that we prepare in the foregoing sections.

The contents are as follows: In Section 2, we give a formulation of projective surfaces and introduce several notions such as Wilczynski frame, Demoulin frame, projectively applicable surfaces and projectively minimal surfaces. This is a short introduction to the projective differential geometry of surfaces. Section 1 is a 
preparation for Section 2. In Section 3, we introduce the notion of line congruence and $W$-congruence, and in Section 6 we formulate a projective theory of line congruences.

In Section 4, we give a summary of Laplace transformations, mainly relying on the books by Darboux. First, in terms of line congruence, the notion of Laplace transformation is introduced and its fundamentals are given. Second, sequences of consecutive Laplace transformations are treated. Third, we turn our attention to the Euler-Darboux-Poisson equation, and we treat the hypergeometric systems by Appell as examples. A characterization of Demoulin surfaces by Godeaux sequences, a classical result, is then presented. Section 5 is a digression to affine spheres. The definition of affine spheres, the differential system describing affine spheres, and properties relative to the Laplace transformation will be given.

A line congruence can be regarded as a surface in the projective 5-space that is the moduli space of projective lines. It lies in a four-dimensional quadratic hypersurface defined by the Plücker relation. A line congruence is said to belong to a linear complex if it lies, regarded as a surface, in a hyperplane. This is explained in Section 7. In Section 8, we treat Laplace transformations of line congruences.

A line congruence is described by a pair of two focal surfaces. In Section 9, the projective invariants of focal surfaces are explicitly given, and a characterization of line congruences for which both focal surfaces are quadrics, found by Wilczynksi, is proved.

There are a number of classical books on projective differential geometry. Among others, the books by Fubini and Čeck [FC1, FC2], the book by Lane [L42], the three-volume book by Bol [Bol], the book on nets by Tzitzeica [Tz24] and the book by Wilczynski [W06] are fundamental. In his book [FC2], Fubini gave a method for constructing $W$-congruences, which will be reproduced here in Section 10. Section 11 treats Demoulin transformations by introducing Demoulin lines and Demoulin congruences. Explicit forms will be given by relying mainly on the works of [Fi30, Su57, Su64]. Section 12 again treats Demoulin transformations intrinsically by appealing to Demoulin coframes.

Section 13 gives a characteristic property of $W$-congruences between projectively minimal surfaces, and presents a transformation formula of projectively minimal surfaces originally due to Marcus [Mar80].

In Appendix A, an example of a line congruence derived from one of Appell's systems is given; in Appendix B, we recall a fundamental formulation of line congruences in the Euclidean 3-space and show that $W$-congruence, or originally Weingarten congruence, comes from the normal congruence associated with Weingarten surfaces. Appendix C gives a proof of a statement in Section 4.8. 


\section{Notation}

$\mathbb{P}^{n}$ denotes the $n$-dimensional projective space with homogeneous coordinates $\left[z^{0}, z^{1}, \ldots, z^{n}\right] . \mathbb{P}_{n}$ denotes its dual projective space. The $\wedge$-product is frequently used in this article. For two vectors $z=\left[z^{0}, z^{1}, \ldots, z^{n}\right]$ and $w=\left[w^{0}, w^{1}, \ldots, w^{n}\right]$, the vector $z \wedge w=\left[z^{i} w^{j}-z^{j} w^{i}\right](0 \leq i \neq j \leq n)$ defines a vector in $\mathbb{P}^{\left(\begin{array}{c}n+1 \\ 2\end{array}\right)-1}$. The multi-wedge product $z \wedge w \wedge \cdots$ is similarly defined. The coefficient number field is $\mathbb{R}$ or $\mathbb{C}$ throughout this article.

\section{Remarks on references}

The books mentioned above are used in several places without mention. The formulation in Section 1 is due to [W06, Sa99], and Section 2 is based on [Sa88, Sa99, Fe00a, FS99, L42]. Formulations in Section 4 are based essentially on [D2], except for some additional matters in Sections 4.7-4.8. We refer the reader to [NS94] for the affine differential geometry described in Section 5. A part of Section 7 and the contents of Sections 8 and 9 depend on [W11]. The material of Section 10 comes from [FC1]; we refer also to [L42]. Section 11 relies on [Fi30]. Section 12 was written in part by using the work of [Su57]. At the end of each section, more information on references will be given.

\section{Ruled surfaces}

A projective surface is an immersion of a two-dimensional manifold into a threedimensional projective space $\mathbb{P}^{3}$. It is given locally by a map $(x, y) \mapsto z(x, y) \in \mathbb{P}^{3}$. To study the immersion, it is fundamental to consider the ruled surface consisting of tangent lines to the family of curves, say $x \mapsto z(x, y)$. In this section, we provide fundamental invariants of ruled surfaces and discuss a property of the Plücker embedding of a ruled surface. We start by recalling the fundamental invariants of space curves.

\subsection{Space curves}

It was G. H. Halphen who developed a projective theory of linear ordinary differential equations by introducing geometric invariants of curves defined by these equations. We give a sketch of his theory for space curves, without proofs. 
Let us consider the linear differential equation

$$
z^{(n+1)}+p_{1} z^{(n)}+\cdots+p_{n} z^{\prime}+p_{n+1} z=0,
$$

where $z(t)$ is an unknown function and $p_{1}, \ldots, p_{n+1}$ are scalar functions of the variable $t$. We choose arbitrarily a set of independent solutions, say $z^{1}(t), \ldots, z^{n+1}(t)$. Then, the map

$$
t \longmapsto\left[z^{1}(t), \ldots, z^{n+1}(t)\right]
$$

defines a curve into the projective space $\mathbb{P}^{n}$. Any other set of solutions defines a curve that is a projective transformation of the original curve. Hence, we can say that the ordinary differential equation above defines a curve uniquely up to a projective transformation. Conversely, given a curve in the projective space $\mathbb{P}^{n}$, we can find an ordinary differential equation satisfied by each coordinate function of the associated mapping. However, the equation is not uniquely defined because the homogeneous coordinates are determined only up to scalar multiplication. We also have the ambiguity in the choice of parameter $t$, when we are starting with only the curve's image in $\mathbb{P}^{n}$. Hence, we can admit a change of variables such as

$$
(z, t) \longmapsto(y=\lambda(t) z, s=f(t))
$$

in order to get geometrical information from the associated curve. This reasoning is essential for developing the projective theory of curves in relation to linear differential equations. Later, this was generalized by Wilczynski to develop the projective differential geometry of submanifolds in relation to linear differential systems.

To illustrate the above reasoning, let us consider a space curve that is a mapping $t \mapsto[z(t)] \in \mathbb{P}^{3}$, where $z(t)$ is a vector in the homogeneous coordinate space of dimension four. At a point of the curve where $\operatorname{det}\left(z^{\prime \prime \prime}, z^{\prime \prime}, z^{\prime}, z\right) \neq 0$, each component is a solution of an ordinary differential equation

$$
z^{\prime \prime \prime \prime}+p_{1} z^{\prime \prime \prime}+p_{2} z^{\prime \prime}+p_{3} z^{\prime}+p_{4} z=0,
$$

where the $p_{j}$ are functions of $t$. By multiplying $z$ by a scalar factor, the equation is changed to the form

$$
z^{\prime \prime \prime \prime}+q_{2} z^{\prime \prime}+q_{3} z^{\prime}+q_{4} z=0 .
$$

Performing the transformation (1.1), the transformed equation has the form

$$
\dddot{y}+\left\{4 \lambda^{\prime} /\left(\lambda f^{\prime}\right)+6 f^{\prime \prime} /\left(f^{\prime}\right)^{2}\right\} \dddot{y}+\cdots=0,
$$

where $\{\cdot\}$ denotes the derivation with respect to $s$. Hence, by choosing $\lambda$ so that $\lambda^{\prime} / \lambda=-(3 / 2) f^{\prime \prime} / f^{\prime}$, we get the equation into the form

$$
\dddot{y}+\left(q_{2}-10\{f ; t\}\right) /\left(f^{\prime}\right)^{2} \ddot{y}+\cdots=0,
$$


where $\{f ; t\}=\left(f^{\prime \prime} / f^{\prime}\right)^{\prime} / 2-\left(f^{\prime \prime} / f^{\prime}\right)^{2} / 4$ is the Schwarzian derivative of $f$ with respect to $t$. We now solve the equation $q_{2}=10\{f ; t\}$ to get a function $f$. Using this $f$, we finally have the equation in the form

$$
\dddot{y}+r_{3} \dot{y}+r_{4} y=0 \text {. }
$$

Here note that the parameter $s$ is not unique, but is determined uniquely up to a fractional linear transformation in view of the fractional invariance of the Schwarzian derivative. This means that the curve has a unique projective structure.

It is known that the two differential forms

$$
\psi_{3}=r_{3} d s^{3}, \quad \psi_{4}=\left(r_{4}-\frac{1}{2} r_{3}^{\prime}\right) d s^{4}
$$

are defined canonically with respect to the first equation (1.2), and are called fundamental invariants of the space curve. Relative to equation (1.3), we see that $\psi_{3}=\left(q_{3}-q_{2}^{\prime}\right) d t^{3}$. When $r_{3} \neq 0$, or equivalently when $q_{3} \neq q_{2}^{\prime}$, we can choose a special parameter $t$ so that $\psi_{3}=d t^{3}$, which is called the projective length parameter of the space curve.

Among several known geometrical interpretations of these invariants, we cite one for later use. At each point of the curve we associate the tangent line that can be regarded as the vector

$$
\xi=y \wedge \dot{y}
$$

in the space $\mathbb{P}^{5} \equiv \stackrel{2}{\wedge} \mathbb{P}^{3}$. By successive differentiation, we get

$$
L:=\xi^{(5)}+r_{3} \ddot{\xi}-2\left(2 r_{4}-\dot{r_{3}}\right) \dot{\xi}-\left(2 \dot{r_{4}}-\ddot{r_{3}}\right) \xi=2 r_{3} \dot{y} \wedge \ddot{y},
$$

and

$$
r_{3} \dot{L}-\dot{r_{3}} L-r_{3}^{2}\left(\dddot{\xi}+r_{3} \xi\right)=0 \text {. }
$$

The last equation defines a sixth-order differential equation relative to $\xi$. However, when $r_{3}=0$ this equation degenerates and $\xi$ satisfies the equation $L=0$, which is a fifth-order equation. Namely, there exists a linear relation amongst the six components of the vector $\xi$.

When the Plücker image of the set of tangent lines in $\mathbb{P}^{5}$ is lying in a hyperplane, then the curve is said to belong to a linear complex. Hence, we can state the following.

Proposition 1.1. (Halphen [Ha83, p. 332]) The space curve defined by (1.3) belongs to a linear complex if and only if $q_{3}=q_{2}^{\prime}$. 


\subsection{Ruled surfaces}

A surface in three-dimensional projective space $\mathbb{P}^{3}$, simply called a projective surface, is an immersion denoted by

$$
(x, y) \longrightarrow z(x, y) \in \mathbb{P}^{3} .
$$

Before entering into the general treatment of projective surfaces in the next section, we recall a projective treatment of ruled surfaces.

We call a one-parameter family of lines in three-dimensional projective space $\mathbb{P}^{3}$ a ruled surface. In practice, it is given by a pair of curves $\left\{z_{1}(x), z_{2}(x)\right\}$ with curve parameter $x$ : we associate a ruled surface with

$$
(x, y) \longrightarrow z(x, y)=z_{1}(x)+y z_{2}(x) .
$$

Here we are regarding any point in $\mathbb{P}^{3}$ as a vector in the homogeneous coordinate space of dimension four. The curves $z_{1}$ and $z_{2}$ are called generating curves.

\section{Example 1.2.}

(1) Let $z_{1}(x)=[1, x, 0,0]$ and $z_{2}(x)=[0,0,1, x]$ in the homogeneous coordinates. Then $z(x, y)=[1, x, y, x y]$, which denotes a quadric $z^{1} z^{4}=z^{2} z^{3}$ relative to the homogeneous coordinates $\left[z^{1}, z^{2}, z^{3}, z^{4}\right]$.

(2) For a given curve $z_{1}(x)$, define a second curve $z_{2}(x)$ by $z_{2}(x)=A+k z_{1}(x)$, where $A$ is a fixed vector and $k$ a scalar. Then the surface is nothing but a cone.

(3) Given a space curve $c(x)$, the pair $\left\{c(x), c^{\prime}(x)\right\}$ defines a ruled surface consisting of tangent lines of the curve $c(x)$, which is called a tangent developable surface.

Definition 1.3. A ruled surface $\left\{z_{1}, z_{2}\right\}$ is said to be developable if

$$
z_{1} \wedge z_{2} \wedge z_{1}^{\prime} \wedge z_{2}^{\prime}=0 .
$$

A developable surface is locally a cone or a tangent developable surface. In the latter case, a generating space curve is called a directrix curve. A directrix curve is written as $c(x)=\alpha z_{1}(x)+\beta z_{2}(x)$ for certain scalars $\alpha(x)$ and $\beta(x)$, with the property that $c^{\prime}(x) \equiv 0\left(\bmod z_{1}, z_{2}\right)$.

In the following, we always assume that the ruled surface is not developable. With this assumption, we will have the following system of differential equations.

$$
z_{i}^{\prime \prime}=\sum_{j} p_{i}^{j} z_{j}^{\prime}+\sum_{j} q_{i}^{j} z_{j}, \quad 1 \leq i, j \leq 2 .
$$

Conversely, a system of this form defines a ruled surface: we can see that this system has four independent solutions that define a pair of curves in $\mathbb{P}^{3}$, which in turn defines a ruled surface. The surface is defined uniquely up to a projective transformation. 
However, the surface itself does not define the system uniquely, it still has the freedom of change of variables and generating curves. In fact, the change of variables $(x, z) \rightarrow(y, w)$ given by

$$
w_{i}=\sum_{j} a_{i}^{j}(x) z_{j}, \quad \operatorname{det}\left(a_{i}^{j}\right) \neq 0
$$

keeps the surface unchanged. With this freedom of choice, let us try to simplify the expression of the system (1.4).

Definition 1.4. Let $z(x, y)$ be a surface. A curve on this surface defined by $x=x(t)$ and $y=y(t)$ is called an asymptotic curve if the four vectors $z, z_{x}, z_{y}$, and $z_{t t}$ are linearly dependent:

$$
z \wedge z_{x} \wedge z_{y} \wedge z_{t t}=0 .
$$

For a ruled surface $z=z_{1}(x)+y z_{2}(x)$, we have

$$
\begin{gathered}
z \wedge z_{x} \wedge z_{y}=z_{1} \wedge z_{1}^{\prime} \wedge z_{2}+y z_{1} \wedge z_{2}^{\prime} \wedge z_{2}, \\
z_{t}=\left(z_{1}^{\prime}+y z_{2}^{\prime}\right) \dot{x}+z_{2} \dot{y}, \\
z_{t t}=\left(z_{1}^{\prime}+y z_{2}^{\prime}\right) \ddot{x}+\left(z_{1}^{\prime \prime}+y z_{1}^{\prime \prime}\right)(\dot{x})^{2}+2 z_{2}^{\prime} \dot{x} \dot{y}+z_{2} \ddot{y},
\end{gathered}
$$

where $\{\cdot\}$ denotes the derivation with respect to $t$. Hence,

$$
z \wedge z_{x} \wedge z_{y} \wedge z_{t t}=2 \dot{x} \dot{y} z_{1} \wedge z_{1}^{\prime} \wedge z_{2} \wedge z_{2}^{\prime}-\dot{x}^{2} A
$$

where

$A=z_{1} \wedge z_{2} \wedge z_{1}^{\prime} \wedge z_{1}^{\prime \prime}+y\left(z_{1} \wedge z_{2} \wedge z_{2}^{\prime} \wedge z_{1}^{\prime \prime}+z_{1} \wedge z_{2} \wedge z_{1}^{\prime} \wedge z_{2}^{\prime \prime}\right)+y^{2} z_{1} \wedge z_{2} \wedge z_{2}^{\prime} \wedge z_{2}^{\prime \prime}$

Therefore, the asymptotic curves are determined by the equation

$$
\dot{x}\left\{2 \dot{y} z_{1} \wedge z_{1}^{\prime} \wedge z_{2} \wedge z_{2}^{\prime}-\dot{x} A\right\}=0,
$$

which always has two different solutions, i.e. two asymptotic curves pass through each point. One of these is a ruling line defined by $\dot{x}=0$ and the other is given by a differential equation of Riccati type

$$
2 z_{1} \wedge z_{1}^{\prime} \wedge z_{2} \wedge z_{2}^{\prime} d y-A d x=0 .
$$

Now we reparametrize the surface assuming that both $z_{1}$ and $z_{2}$ are asymptotic curves. In this case we see that

$$
z_{1} \wedge z_{2} \wedge z_{1}^{\prime} \wedge z_{1}^{\prime \prime}=z_{1} \wedge z_{2} \wedge z_{2}^{\prime} \wedge z_{2}^{\prime \prime}=0
$$


which implies that $p_{1}^{2}=p_{2}^{1}=0$ in the system (1.4). We next replace $z_{1}$ and $z_{2}$ by their scalar multiples $\lambda z_{1}$ and $\mu z_{2}$. Then the coefficients $p_{1}^{1}$ and $p_{2}^{2}$ are changed by adding $\lambda^{\prime} / \lambda$ and $\mu^{\prime} / \mu$, respectively. So we can always find $\lambda$ and $\mu$ so that $p_{1}^{1}=p_{2}^{2}=0$. Hence, we have proved the following.

Proposition 1.5. A non-developable ruled surface is given by a system of differential equations

$$
\begin{aligned}
& z_{1}^{\prime \prime}=p z_{1}+q z_{2} \\
& z_{2}^{\prime \prime}=r z_{1}+s z_{2}
\end{aligned}
$$

Example 1.6.

(1) Any non-degenerate quadric is a ruled surface given by the system

$$
z_{1}^{\prime \prime}=z_{2}^{\prime \prime}=0 .
$$

(2) Cayley's cubic scroll is by definition

$$
\left(z^{2}\right)^{3}+z^{1}\left(z^{1} z^{4}+z^{2} z^{3}\right)=0,
$$

relative to the coordinates $\left[z^{1}, z^{2}, z^{3}, z^{4}\right]$. It is ruled by two generating curves $z_{1}=\left[1,-x,-x^{2}, 0\right]$ and $z_{2}=[0,0,1, x]$. Hence, the system of equations is

$$
z_{1}^{\prime \prime}=-2 z_{2}+2 x z_{2}^{\prime}, \quad z_{2}^{\prime \prime}=0 .
$$

If we set

$$
z_{3}=z_{1}-\frac{1}{2} x^{2} z_{2}+a z_{2}=\left[1,-x,-\frac{3}{2} x^{2}+a,-\frac{1}{2} x^{3}+a x\right],
$$

where $a$ is any constant, then the system is written in the form asserted in the proposition:

$$
z_{3}^{\prime \prime}=-3 z_{2}, \quad z_{2}^{\prime \prime}=0
$$

\subsection{Fundamental invariants of a ruled surface}

Let us check the covariance of the system (1.6) relative to the transformation (1.5). Setting, for simplicity,

$$
X=\left(\begin{array}{l}
z_{1} \\
z_{2}
\end{array}\right), \quad Q=\left(\begin{array}{ll}
p & q \\
r & s
\end{array}\right),
$$


the system is written as

$$
X^{\prime \prime}=Q X
$$

Relative to the transformation $y=f(x)$ and $Y=A X$, where $A=\left(a_{i}^{j}\right)$, the variable $Y$ satisfies

$$
\left(f^{\prime}\right)^{2} \ddot{Y}=\left(2 f^{\prime} A^{\prime} A^{-1}-f^{\prime \prime}\right) \dot{Y}+\left(A^{\prime \prime} A^{-1}+A Q A^{-1}-2 A^{\prime} A^{-1} A^{\prime} A^{-1}\right) Y,
$$

where $\left\{{ }^{\circ}\right\}=d / d y$ and $\left\{^{\prime}\right\}=d / d x$. Then $B=\left(f^{\prime}\right)^{-1 / 2} A$ should satisfy $B^{\prime}=0$ in order that the coefficient of $\dot{Y}$ vanishes. In this case, the system becomes

$$
\left(f^{\prime}\right)^{2} \ddot{Y}=\left(2\{f ; x\}+B Q B^{-1}\right) Y,
$$

where $\{f ; x\}$ is the Schwarzian derivative of $f$ relative to the variable $x$. Since the trace of the coefficient matrix of $Y$ is $\operatorname{tr} Q+4\{f ; x\}$, we can assume this coefficient vanishes by choosing $f$ appropriately. Then any transformation preserving this condition is of the form

$$
A=\left(f^{\prime}\right)^{1 / 2} B, \quad\{f ; x\}=0 ;
$$

that is,

$$
f=\frac{\alpha x+\beta}{\gamma x+\delta}, \quad A=(\gamma x+\delta)^{-1} B, \quad \text { where } B \text { is a constant matrix. }
$$

The matrix-valued quadratic form $Q d x^{2}$ changes under this transformation as

$$
Q d x^{2} \longrightarrow B\left(Q d x^{2}\right) B^{-1} .
$$

Definition 1.7. We call $Q d x^{2}$ the fundamental invariant of a ruled surface.

Note that the condition $Q=0$ holds only if the ruled surface is a quadric; see Example 1.6. Summarizing the argument, we have seen the following theorem.

THEOREM 1.8.

(1) Any ruled surface can be written as a solution of a system of the form

$$
X^{\prime \prime}=Q X ; \quad X={ }^{\mathrm{t}}\left(z_{1}, z_{2}\right), \quad \operatorname{tr} Q=0 .
$$

(2) The transformation (1.5) preserving this normalization is given by (1.7) and the conjugate class of the matrix-valued quadratic form $Q d x^{2}$ is invariant; in particular, $(\operatorname{det} Q) d x^{4}$ is an absolute invariant. 
(3) For two ruled surfaces given, respectively, by curves $\left(z_{1}(x), z_{2}(x)\right)$ and curves $\left(w_{1}(y), w_{2}(y)\right)$, let $Q d x^{2}$ and $R d y^{2}$ denote the fundamental invariants. If both are projectively equivalent, then there exists a diffeomorphism between the parameters, $y=f(x)$, and a non-singular constant matrix $B$ such that

$$
f^{*}\left(R d y^{2}\right)=B\left(Q d x^{2}\right) B^{-1} .
$$

Conversely, if there exists a mapping $f$ and a matrix $B$ satisfying this identity, then the ruled surfaces $z$ and $w$ are projectively equivalent.

Remark 1.9. As the parameter is determined up to a fractional linear transformation, we can say that any ruled surface has a one-dimensional projective structure.

\subsection{Differential invariants of a ruled surface}

Let $I_{x}$ be a differential polynomial defined by using the matrix $Q$ relative to the variable $x$. With $I_{y}$ denoting the polynomial of the same form relative to $y$, we say $I$ is an invariant of weight $k$ if

$$
I_{y}=I_{x}\left(f^{\prime}\right)^{-k}
$$

The following is a list of some examples of such differential invariant polynomials (we refer the reader to [W06]):

$$
\begin{gathered}
\theta_{4}=-4 \operatorname{det} Q, \\
\theta_{6}=9 \operatorname{det}\left(Q^{\prime}\right)-2(\operatorname{det} Q)^{\prime \prime}, \\
\theta_{10}=-4 \operatorname{det} Q \operatorname{det}\left(Q^{\prime}\right)+\left((\operatorname{det} Q)^{\prime}\right)^{2}, \\
\theta_{9}=\operatorname{det}\left(\begin{array}{ccc}
p & q & r \\
p^{\prime} & q^{\prime} & r^{\prime} \\
p^{\prime \prime} & q^{\prime \prime} & r^{\prime \prime}
\end{array}\right) .
\end{gathered}
$$

The weights are 4, 6, 10 and 9 , respectively.

As we have done for space curves, let us consider the Plücker coordinates of the line $\overline{z_{1} z_{2}}$ joining the points $z_{1}$ and $z_{2}$ represented by the vector $\xi=z_{1} \wedge z_{2}$. Its components are $\xi_{i j}=z_{1}^{i} z_{2}^{j}-z_{1}^{j} z_{2}^{i}$, where $z_{k}=\left[z_{k}^{1}, z_{k}^{2}, z_{k}^{3}, z_{k}^{4}\right]$. Then the point $\xi$ lies on the quadratic hypersurface in $\mathbb{P}^{5}$ determined by the Plücker relation

$$
\xi_{12} \xi_{34}-\xi_{13} \xi_{24}+\xi_{14} \xi_{23}=0 .
$$

Furthermore, we have the following. 
PROPOSITION 1.10. The invariant $\theta_{9}$ vanishes if and only if $\xi$ lies in a hyperplane.

Proof. Differentiating $\xi=z_{1} \wedge z_{2}$, we get $\xi^{\prime}=z_{1}^{\prime} \wedge z_{2}+z_{1} \wedge z_{2}^{\prime}$ and $\xi^{\prime \prime}=2 z_{1}^{\prime} \wedge z_{2}^{\prime}$. Then, it is easy to see the following:

$$
\begin{gathered}
\frac{1}{2} \xi^{\prime \prime \prime}+p \xi^{\prime}=2 p z_{1} \wedge z_{2}^{\prime}+q z_{2} \wedge z_{2}^{\prime}-r z_{1} \wedge z_{1}^{\prime}, \\
\frac{1}{2} \xi^{\prime \prime \prime \prime}+p^{\prime} \xi^{\prime}+2\left(p^{2}+q r\right) \xi=2 p^{\prime} z_{1} \wedge z_{2}^{\prime}+q^{\prime} z_{2} \wedge z_{2}^{\prime}-r^{\prime} z_{1} \wedge z_{1}^{\prime}, \\
\frac{1}{2} \xi^{(5)}+\left(p^{\prime \prime}+2\left(p^{2}+q r\right)\right) \xi^{\prime}+3\left(p^{2}+q r\right)^{\prime} \xi=2 p^{\prime \prime} z_{1} \wedge z_{2}^{\prime}+q^{\prime \prime} z_{2} \wedge z_{2}^{\prime}-r^{\prime \prime} z_{1} \wedge z_{1}^{\prime} .
\end{gathered}
$$

Hence, $\xi$ satisfies a fifth-order equation if and only if the right-hand sides of the above equations are linearly dependent, namely, if $\theta_{9}$ vanishes.

Remark 1.11. Investigation of the fundamental invariants of curves in projective space was done by Laguerre and Forsyth. A modern treatment was given by Se-ashi [Se87]. For more details regarding the contents of this section, we refer the reader to [W06, Sa99].

\section{Projective theory of surfaces}

This section aims at describing the theory of surfaces in the three-dimensional projective space and introduces some typical classes of surfaces, such as Demoulin surfaces, projectively applicable surfaces and projectively minimal surfaces. To define invariants appropriately, we need to associate special frames to the surfaces, called normalized frames. The normalization process works also in higher dimensions, and we start with a description of invariants on hypersurfaces.

\subsection{Projective invariants of hypersurfaces}

Let $e_{0}: M^{n} \rightarrow \mathbb{P}^{n+1}$ be an immersion of an $n$-dimensional manifold, which defines a hypersurface. We identify it with its (arbitrary) lift to $\mathbb{R}^{n+2}-\{0\}$ or $\mathbb{C}^{n+2}-\{0\}$. We choose linearly independent tangent vector fields to $e_{0}(M)$ denoted by $e_{1}, \ldots, e_{n}$, which are locally defined on $M$ and linearly independent of $e_{0}$. Let $e_{n+1}$ be a vector field, also defined locally, which are linearly independent of $e_{0}, e_{1}, \ldots, e_{n}$. The ordered set $e=\left(e_{0}, e_{1}, \ldots, e_{n}, e_{n+1}\right)$ is called a (projective) frame along the immersion. It satisfies a differential equation

$$
d e=\omega e, \quad \text { i.e., } d e_{\alpha}=\sum_{\beta} \omega_{\alpha}^{\beta} e_{\beta},
$$


thus defining an $(n+2) \times(n+2)$-matrix valued 1 -form $\omega$. Here and in the following, the index range of Greek letters is from 0 to $n+1$ and the index range of roman letters is from 1 to $n$. The form $\omega$ satisfies the integrability condition

$$
d \omega=\omega \wedge \omega .
$$

If we consider only frames $e$ with the property $\operatorname{det}\left(e_{0}, e_{1}, \ldots, e_{n}, e_{n+1}\right)=1$, then it also holds that

$$
\sum_{\alpha} \omega_{\alpha}^{\alpha}=0,
$$

which we assume in the following. When necessary, we will denote $\omega(e)$ for the 1 -form $\omega$ corresponding to a given frame $e$. Two frames, say $e$ and $\tilde{e}$, are related by

$$
\tilde{e}=g e,
$$

where $g$ is a mapping (locally) from $M$ to the projective linear group $P G L_{n+2}$. Then, we see that

$$
\omega(\tilde{e})=d g g^{-1}+g \omega(e) g^{-1} .
$$

We now further restrict the choice of $e$. We first look at the condition $\omega_{0}^{n+1}=0$, which follows from the definition of $e_{n+1}$. By taking exterior derivation, we get

$$
\sum_{i=1}^{n} \omega^{i} \wedge \omega_{i}^{n+1}=0,
$$

where $\omega^{i}=\omega_{0}^{i}$; by definition, these are independent basic forms. We can write $\omega_{i}^{n+1}=\sum_{j} h_{i j} \omega^{j}$ for a symmetric tensor $h_{i j}$ and define a symmetric 2 -form

$$
\varphi_{2}=\sum_{i, j} h_{i j} \omega^{i} \omega^{j}
$$

The conformal class of $\varphi_{2}$ can be seen to be unique. In order to see this, we need to know how the invariants transform under a change of the frame $e$ to a new frame $\tilde{e}=g e$, where $g$ is given by

$$
g=\left(\begin{array}{lll}
\lambda & 0 & 0 \\
b & a & 0 \\
\mu & c & \nu
\end{array}\right) .
$$

By the assumption made above, $\operatorname{det} g=1$. Let us denote by adding tildes to the invariants relative to the frame $\tilde{e}$ and let $A$ denote the inverse matrix of $a$. Then, a calculation using (2.2) shows that

$$
\tilde{\omega}^{i}=\lambda \sum_{j} A_{j}^{i} \omega^{j} \quad \text { and } \quad \tilde{\omega}_{i}^{n+1}=v^{-1} \sum_{k} a_{i}^{k} \omega_{k}^{n+1},
$$


from which we obtain

$$
\tilde{h}=(\lambda v)^{-1} a h^{\mathrm{t}} a,
$$

where $h=\left(h_{i j}\right)$. Hence, we see that $\tilde{\varphi}_{2}=\lambda \nu^{-1} \varphi_{2}$. We say that the immersion $e_{0}$ is non-degenerate when the matrix $\left(h_{i j}\right)$ is non-singular; we assume this property in the following. Then, equation (2.3) implies that there exists a frame such that $\left|\operatorname{det} h_{i j}\right|=1$. In particular, $|\lambda \nu|=1$. The formula (2.2) shows that

$$
\tilde{\omega}_{0}^{0}+\tilde{\omega}_{n+1}^{n+1}=\omega_{0}^{0}+\omega_{n+1}^{n+1}+v^{-1} \sum_{i} c^{i} \omega_{i}^{n+1}-\sum_{i, j} b_{i} A_{j}^{i} \omega^{j}
$$

and we can assume $\omega_{0}^{0}+\omega_{n+1}^{n+1}=0$ and then, $b$ and $c$ are related by

$$
b=v^{-1} a h^{\mathrm{t}} c \text {. }
$$

The exterior derivation of $\omega_{0}^{0}+\omega_{n+1}^{n+1}=0$ gives

$$
\left(\sum_{j=1}^{n} h_{i j} \omega_{n+1}^{j}-\omega_{i}^{0}\right) \wedge \omega^{i}=0 .
$$

Hence, we can define $\ell_{i j}$ so that

$$
\sum_{j=1}^{n} h_{i j} \omega_{n+1}^{j}-\omega_{i}^{0}=\sum_{j=1}^{n} \ell_{i j} \omega^{j} ; \quad \ell_{i j}=\ell_{j i} .
$$

We set

$$
\ell=\frac{1}{n} \sum_{i, j} h^{i j} \ell_{i j}
$$

where $\left(h^{i j}\right)$ is the inverse of the matrix $h=\left(h_{i j}\right)$.

We will next treat the third-order information of the immersion. Define $h_{i j k}$ by the equation

$$
\sum_{k} h_{i j k} \omega^{k}=d h_{i j}-\sum_{k} h_{k j} \omega_{i}^{k}-\sum_{k} h_{i k} \omega_{j}^{k} .
$$

It is seen that $h_{i j k}$ is symmetric relative to all indices and satisfies the so-called apolarity condition:

$$
\sum_{i, j} h^{i j} h_{i j k}=0
$$

for each $k$, which follows from the condition $|\operatorname{det} h|=1$. We define a symmetric cubic form by

$$
\varphi_{3}=\sum_{i, j, k} h_{i j k} \omega^{i} \omega^{j} \omega^{k}
$$


and a scalar called the Fubini-Pick invariant by

$$
F=\sum_{i, j, k, p, q, r} h_{i j k} h_{p q r} h^{i p} h^{j q} h^{k r} .
$$

The cubic form has the invariance

$$
\lambda^{2} v \tilde{h}_{i j k}=h_{p q r} a_{i}^{p} a_{j}^{q} a_{k}^{r}
$$

namely,

$$
\tilde{\varphi}_{3}=\lambda \nu^{-1} \varphi_{3} .
$$

Together with the invariance (2.3) we can conclude that $F \varphi_{2}$ is an absolutely-invariant 2 -form, which is called the projective metric form. We lastly set

$$
\omega_{n+1}^{0}=-\sum_{j} \rho_{j} \omega^{j}
$$

Thus, we have quantities $\left\{h_{i j}, h_{i j k}, \ell_{i j}, \rho_{j}\right\}$. These are canonically defined for a nondegenerate hypersurface and define invariants in the following sense. Continuing the computation of the form $\tilde{\omega}$, we can see the formula

$$
\lambda^{2} \tilde{\ell}_{i j}=\sum_{p, q} a_{i}^{p} \ell_{p q} a_{j}^{q}+\left(2 \mu-v^{-1} c h^{\mathrm{t}} c\right) h_{i j}-v^{-1} \sum_{p, q, r} h_{p q r} c^{p} a_{i}^{q} a_{j}^{r},
$$

from which we get

$$
\lambda \tilde{\ell}=\lambda^{-1} \ell+\left(2 \mu-v^{-1} \operatorname{ch}^{\mathrm{t}} c\right) .
$$

Hence, we can find a frame so that $\ell=0$.

Summarizing the argument above, we have seen the following.

PROPOSITION 2.1.

(1) There exists a frame $e$ with the properties

$$
\operatorname{det}(e)=1, \quad \omega_{0}^{n+1}=0, \quad \omega_{0}^{0}+\omega_{n+1}^{n+1}=0, \quad\left|\operatorname{det} h_{i j}\right|=1 \quad \text { and } \quad \ell=0 .
$$

(2) For such two frames, the connecting transformation $g$ has the form

$$
\left(\begin{array}{ccc}
\lambda & 0 & 0 \\
b & a & 0 \\
\mu & c & \nu
\end{array}\right) \quad \begin{gathered}
|\lambda \nu|=1 \\
\quad \mu=(1 / 2) v^{-1} a h^{\mathrm{t}} c h^{\mathrm{t}} c,
\end{gathered}
$$

where $a$ is an $n \times n$-matrix of $|\operatorname{det} a|=1$.

The second property implies that to any non-degenerate hypersurface is associated canonically a conformal connection.

Further, the invariance is summarized in the following way. 
Proposition 2.2. Let $\left\{\tilde{h}_{i j}, \tilde{h}_{i j k}, \tilde{\ell}_{i j}, \tilde{\rho}_{j}\right\}$ be such invariants defined for the frame ge, where $g$ is a matrix given in Proposition 2.1(2) with components $\lambda$, $v$, and $a=\left(a_{i}^{j}\right)$ and $c=\left(c^{i}\right)$. Set

$$
\begin{aligned}
& \rho=\sum \rho_{i} \omega^{i}, \quad \mathcal{L}=\sum \ell_{i j} \omega^{i} \omega^{j}, \quad \mathcal{L}(c)=\sum \ell_{i j} \omega^{i} c^{j} / \nu, \\
& H(c)=\sum h_{i j k} \omega^{i} \omega^{j} c^{k} / \nu, \quad H(c, c)=\sum h_{i j k} \omega^{i} c^{j} c^{k} / \nu^{2} .
\end{aligned}
$$

Then the following transformation formulas hold:

$$
\begin{gathered}
\tilde{\varphi}_{2}=\lambda v^{-1} \varphi_{2}, \quad \tilde{\varphi}_{3}=\lambda v^{-1} \varphi_{3}, \\
\tilde{\mathcal{L}}=\mathcal{L}-H(c), \quad \lambda v^{-1} \tilde{\rho}=\rho+\mathcal{L}(c)-\frac{1}{2} H(c, c) .
\end{gathered}
$$

It can be proved that the set of invariants $\left\{h_{i j}, h_{i j k}, \ell_{i j}, \rho_{j}\right\}$ determines the immersion up to a projective transformation. In the case $n \geq 3$, the quantities $\ell_{i j}$ and $\rho_{j}$ are given in terms of $h_{i j}, h_{i j k}$ and their derivatives. However, in the case $n=2$, the situation is different (see Section 2.6).

The invariance in Proposition 2.1 means geometrically the following. Any point $P$ in the space can be written as

$$
P=p^{0} e_{0}+p^{1} e_{1}+\cdots+p^{n} e_{n}+p^{n+1} e_{n+1}
$$

and thus we define the coordinates $p=\left(p^{0}, p^{1}, \ldots, p^{n}, p^{n+1}\right)$ relative to the frame $e: P=p e$. We then set

$$
H=\left(\begin{array}{ccc}
0 & 0 & 1 \\
0 & -h & 0 \\
1 & 0 & 0
\end{array}\right)
$$

and define a quadratic hypersurface as the set $Q=\{P \mid X(P)=0\}$, where $X(P)=p H^{\mathrm{t}} p$. For the frame $\tilde{e}$, we see $P=\tilde{p} \tilde{e}$, where $p=\tilde{p} g$. It is easy to see $g H^{\mathrm{t}} g= \pm \tilde{H}$ in view of the invariance (2.3) and (2.4). This implies that the quadratic hypersurface $Q$ is well-defined independent of frames. It is called the Lie quadratic hypersurface and, by definition, tangent to the given hypersurface up to the second order.

We next see how the Lie quadratic hypersurface $Q$ depends on the point of the given hypersurface. We fix a point $P$ and consider it a function on the given hypersurface. As it does not move, $d P$ is proportional to $P$ itself, which means that $d p=-p \omega-\kappa p$ for some 1-form $\kappa$. Hence, we can see that $d X=p \Omega^{\mathrm{t}} p-2 \kappa p H^{\mathrm{t}} p$, where

$$
\Omega=\sum_{k} H_{k} \omega^{k}, \quad H_{k}=\left(\begin{array}{ccc}
0 & 0 & 0 \\
0 & -h_{i j k} & \ell_{i k} \\
0 & \ell_{j k} & 2 \rho_{k}
\end{array}\right) .
$$


We now assume that $\varphi_{3}=0$. Then, the exterior derivation of (2.6) shows that $\ell_{i j}=0$ by use of the integrability condition (2.1) and then the exterior derivation of (2.5) when $\ell_{i j}=0$ shows that $\rho_{i}=0$. Hence, $d X(P)=0$ for the point $P$ on $Q$, which means that the quadratic hypersurface remains unchanged; namely, we have the following.

THEOREM 2.3. Assume that $\varphi_{2}$ is non-degenerate and $\varphi_{3}=0$. Then the hypersurface is projectively equivalent to a quadratic hypersurface.

Given an immersion $e_{0}: M^{n} \rightarrow \mathbb{P}^{n+1}$ and an associated projective frame $e=\left(e_{0}, e_{1}, \ldots, e_{n}, e_{n+1}\right)$, we define the dual frame $E=\left\{E^{0}, E^{1}, \ldots, E^{n+1}\right\}$ by

$$
E^{\alpha}=(-1)^{\alpha} e_{0} \wedge \cdots \wedge \check{e}_{\alpha} \wedge \cdots \wedge e_{n+1} .
$$

We have $\left\langle e_{\alpha}, E^{\beta}\right\rangle=\delta_{\alpha}^{\beta}$. The frame $E$ satisfies the equation $d E=-E \omega$. The vector $E^{n+1}$ can be identified with the tangent hyperplane of the given hypersurface and also can be regarded as an immersion into the dual projective space, called the dual immersion. A projective frame associated to this dual immersion is given by $\check{E}=\left(E^{n+1}, E^{1}, \ldots, E^{n}, E^{0}\right)$, and if we set $d \check{E}=\Omega \check{E}$, then $\Omega^{i}=-\omega_{i}^{n+1}$, $\Omega_{i}^{n+1}=-\omega^{i}, \Omega_{i}^{j}=-\omega_{i}^{j}, \Omega_{0}^{j}=-\omega_{n+1}^{j}, \omega_{n+1}^{j}=-\omega_{j}^{0}$ and $\Omega_{n+1}^{0}=-\omega_{n+1}^{0}$. When we denote the invariants of the dual immersion by adding asterisks, we can see that $h_{i j}^{*}=h^{i j}, h_{i j k}^{*}=\sum h^{i p} h^{j q} h^{k r} h_{p q r}, \ell_{i j}^{*}=-\sum h^{i p} h^{j q} \ell_{p q}$, and $\rho_{i}^{*}=\sum h^{i p} \rho_{p}$. Consequently $\varphi_{2}^{*}=\varphi_{2}$ and $\varphi_{3}^{*}=\varphi_{3}$.

Remark 2.4. For the Lie quadratic hypersurface, we refer to [Bol, Vol. 3, p. 438]. The case when $n=2$ will be considered again in Section 11.2. For further details on the contents of this section, we refer to [Sa88, Sa99].

\subsection{Projective invariants of surfaces}

Let us consider the case $n=2$. We start with the system defined by an immersion

$$
z:(x, y) \longrightarrow z(x, y) \in \mathbb{P}^{3} .
$$

Assume that the vector $z_{x y}$ considered as a mapping to $\mathbb{R}^{4}-\{0\}$ or $\mathbb{C}^{4}-\{0\}$ is linearly independent of $z, z_{x}$ and $z_{y}$. Then the system has the form

$$
z_{x x}=\ell z_{x y}+a z_{x}+b z_{y}+p z, \quad z_{y y}=m z_{x y}+c z_{x}+d z_{y}+q z .
$$


Relative to a frame $\left\{e_{0}=z, e_{1}=z_{x}, e_{2}=z_{y}, e_{3}=z_{x y}\right\}$, the coframe has the form

$$
\omega=\left(\begin{array}{cccc}
0 & d x & d y & 0 \\
p d x & a d x & b d x & \ell d x+d y \\
q d y & c d y & d d y & d x+m d y \\
* & * & * & *
\end{array}\right) .
$$

Hence, we see that

$$
\varphi_{2}=\ell d x^{2}+2 d x d y+m d y^{2} .
$$

In the following, we assume $1-\ell m \neq 0$ so that $\varphi_{2}$ is non-degenerate. If the coordinates are chosen to be conjugate relative to $\varphi_{2}$ (see Section 4.1), then we get $\ell=m=0$ and the system is reduced to

$$
z_{x x}=a z_{x}+b z_{y}+p z, \quad z_{y y}=c z_{x}+d z_{y}+q z .
$$

Here in the real case, we have assumed that $\varphi_{2}$ is indefinite. The associated coframe is

$$
\omega=\left(\begin{array}{cccc}
0 & d x & d y & 0 \\
p d x & a d x & b d x & d y \\
q d y & c d y & d d y & d x \\
\left(b q+p_{y}\right) d x & \left(a_{y}+b c\right) d x & \left(b d+b_{y}+p\right) d x & a d x+d d y \\
+\left(c p+q_{x}\right) d y & +\left(a c+c_{x}+q\right) d y & +\left(b c+d_{x}\right) d y &
\end{array}\right) .
$$

In particular, we have $d\left(e_{0} \wedge e_{1} \wedge e_{2} \wedge e_{3}\right)=2(a d x+d d y) e_{0} \wedge e_{1} \wedge e_{2} \wedge e_{3}$. Hence, $a d x+d d y$ is an exact form and there exists a function $\theta$ such that $a=\theta_{x}$ and $d=\theta_{y}$. Then,

$$
z_{x x}=\theta_{x} z_{x}+b z_{y}+p z, \quad z_{y y}=c z_{x}+\theta_{y} z_{y}+q z .
$$

The integrability condition of the system is $d \omega=\omega \wedge \omega$, which consists of three equations:

$$
\begin{gathered}
L_{y}=-2 b c_{x}-c b_{x}, \\
M_{x}=-2 c b_{y}-b c_{y}, \\
b M_{y}+2 M b_{y}+b_{y y y}=c L_{x}+2 L c_{x}+c_{x x x},
\end{gathered}
$$

where $L$ and $M$ are traditional notation defined by

$$
L=\theta_{x x}-\frac{1}{2} \theta_{x}^{2}-b \theta_{y}-b_{y}-2 p, \quad M=\theta_{y y}-\frac{1}{2} \theta_{y}^{2}-c \theta_{x}-c_{x}-2 q .
$$

We can simplify the system (2.8) by replacing $e^{-\theta / 2} z$ with $w$; then we get

$$
w_{x x}=\bar{b} w_{y}+\bar{p} w, \quad w_{y y}=\bar{c} w_{x}+\bar{q} w,
$$


where

$$
\bar{b}=b, \quad \bar{c}=c, \quad \bar{p}=p-\frac{1}{2} \theta_{x x}+\frac{1}{4} \theta_{x}^{2}+\frac{1}{2} b \theta_{y}, \quad \bar{q}=q-\frac{1}{2} \theta_{y y}+\frac{1}{4} \theta_{y}^{2}+\frac{1}{2} c \theta_{x} .
$$

The invariants $L$ and $M$ remain the same under this change of the unknown from $z$ to $w$.

The dual surface is defined by the immersion $\xi=z \wedge z_{x} \wedge z_{y}$. It satisfies the system

$$
\xi_{x x}=\theta_{x} \xi_{x}-b \xi_{y}+\bar{p} \xi, \quad \xi_{y y}=-c \xi_{x}+\theta_{y} \xi_{y}+\bar{q} \xi
$$

where

$$
\bar{p}=p+b_{y}+b \theta_{y}, \quad \bar{q}=q+c_{x}+c \theta_{x} .
$$

\subsection{The Wilczynski frame of a surface}

In this section, we treat the system

$$
z_{x x}=b z_{y}+p z, \quad z_{y y}=c z_{x}+q z
$$

which we call the canonical system of the given immersion. Relative to this system, the integrability condition (2.9) simplifies to

$$
\begin{gathered}
p_{y}=b c_{x}+\frac{1}{2} b_{x} c-\frac{1}{2} b_{y y}, \quad q_{x}=c b_{y}+\frac{1}{2} b c_{y}-\frac{1}{2} c_{x x}, \\
b_{y y y}-b c_{x y}-2 b q_{y}-2 b_{y} c_{x}-4 q b_{y}=c_{x x x}-c b_{x y}-2 c p_{x}-2 b_{x} c_{y}-4 p c_{x} .
\end{gathered}
$$

We now choose a frame defined by

$$
e_{0}=z, \quad e_{1}=z_{x}, \quad e_{2}=z_{y}, \quad e_{3}=z_{x y}-\frac{1}{2} b c z
$$

Then the coframe $\omega$ for the canonical system (2.12) is

$$
\omega=\left(\begin{array}{cccc}
0 & d x & d y & 0 \\
p d x+\frac{1}{2} b c d y & 0 & b d x & d y \\
q d y+\frac{1}{2} b c d x & c d y & 0 & d x \\
\left(b q+p_{y}\right) d x+\left(c p+q_{x}\right) d y & \left(q+c_{x}\right) d y & \left(p+b_{y}\right) d x & 0 \\
-\frac{1}{2} d(b c) & +\frac{1}{2} b c d x & +\frac{1}{2} b c d y
\end{array}\right) .
$$


From this expression, we have

$$
\begin{gathered}
h_{11}=h_{22}=0, \quad h_{12}=h_{21}=1, \\
h_{111}=-2 b, \quad h_{222}=-2 c, \quad h_{112}=h_{122}=0, \\
F=8 b c, \\
\ell_{11}=b_{y}, \quad \ell_{12}=\ell_{21}=0, \quad \ell_{22}=c_{x}, \\
\rho_{1}=\frac{1}{2}(b c)_{x}-b q-p_{y}, \quad \rho_{2}=\frac{1}{2}(b c)_{y}-c p-q_{x} .
\end{gathered}
$$

In particular, the condition $b=c=0$ is necessary and sufficient for the surface to be quadratic. The surface is ruled if and only if $F=0$, i.e. $b c=0$. In fact, assume that $c=0$. Then by (2.13), $q$ is independent of $x$ and we may assume that $q=0$ by multiplying $z$ by some factor. Checking again (2.13), it is seen that the system has the form

$$
z_{x x}=\left(\alpha y^{2}+\beta y+\gamma\right) z_{y}+(-\alpha y+\delta) z, \quad z_{y y}=0,
$$

where $\alpha, \beta, \gamma$ and $\delta$ are functions of $x$. This shows that $z$ is linear relative to $y$ and has the form $u(x)+y v(x)$, where $u$ and $v$ are solutions of the system $u_{x x}=\delta u+\gamma v$ and $v_{x x}=-\alpha u+(\beta+\delta) v$; thus, $z$ defines a ruled surface. The converse is shown similarly.

In some cases, it is useful to adopt another frame $\left\{z, z_{1}, z_{2}, \eta\right\}$ as far as $b c \neq 0$, called the Wilczynski frame, defined by

$$
\begin{gathered}
z=z, \quad z_{1}=z_{x}-\frac{c_{x}}{2 c} z, \quad z_{2}=z_{y}-\frac{b_{y}}{2 b} z, \\
\eta=z_{x y}-\frac{c_{x}}{2 c} z_{y}-\frac{b_{y}}{2 b} z_{x}+\left(\frac{b_{y} c_{x}}{4 b c}-\frac{1}{2} b c\right) z .
\end{gathered}
$$

We introduce notation

$$
\kappa_{1}=\frac{b c-(\log b)_{x y}}{2}, \quad \kappa_{2}=\frac{b c-(\log c)_{x y}}{2},
$$

and

$$
P=p+\frac{b_{y}}{2}-\frac{c_{x x}}{2 c}+\frac{c_{x}^{2}}{4 c^{2}}, \quad Q=q+\frac{c_{x}}{2}-\frac{b_{y y}}{2 b}+\frac{b_{y}^{2}}{4 b^{2}} .
$$


Then the system (2.12) can be written in the Pfaffian form

$$
\begin{aligned}
d\left(\begin{array}{c}
z \\
z_{1} \\
z_{2} \\
\eta
\end{array}\right)= & {\left[\left(\begin{array}{cccc}
\frac{c_{x}}{2 c} & 1 & 0 & 0 \\
P & -\frac{c_{x}}{2 c} & b & 0 \\
\kappa_{1} & 0 & \frac{c_{x}}{2 c} & 1 \\
b Q & \kappa_{1} & P & -\frac{c_{x}}{2 c}
\end{array}\right) d x\right.} \\
& \left.\left.+\left(\begin{array}{cccc}
\frac{b_{y}}{2 b} & 0 & 1 & 0 \\
\kappa_{2} & \frac{b_{y}}{2 b} & 0 & 1 \\
Q & c & -\frac{b_{y}}{2 b} & 0 \\
c P & Q & \kappa_{2} & -\frac{b_{y}}{2 b}
\end{array}\right)\right] d y\right]\left(\begin{array}{c}
z \\
z_{1} \\
z_{2} \\
\eta
\end{array}\right) .
\end{aligned}
$$

For this expression of the coframe, the invariants are given as follows:

$$
\begin{gathered}
h_{11}=h_{22}=0, \quad h_{12}=h_{21}=1, \\
h_{111}=-2 b, \quad h_{222}=-2 c, \quad h_{112}=h_{122}=0, \\
\ell_{11}=\ell_{12}=\ell_{21}=\ell_{22}=0, \quad \rho_{1}=-b Q, \quad \rho_{2}=-c P .
\end{gathered}
$$

One merit of using the Wilczynski frame is that we can suppose that all $\ell_{i j}=0$. This is due to the fact that the dimension is two.

\subsection{Demoulin frames of a surface}

According to the invariance stated in Proposition 2.1, the expressions of invariants are dependent on the frame. In this section, we continue the procedure in Section 2.1 and try to find a frame so that $\rho_{i}=0$.

To simplify notation, we assume that the surface is indefinite and choose a frame with the properties in Proposition 2.1(1) so that

$$
h=\left(\begin{array}{ll}
0 & 1 \\
1 & 0
\end{array}\right) .
$$

Then, the apolarity condition and the condition $\ell=0$ imply that $h_{112}=h_{122}=0$ and $\ell_{12}=0$. Referring to the choice of frames in the previous section, we set $h_{111}=-2 b$ and $h_{222}=-2 c$. Then the transformation rule of Proposition 2.1 applied to the 
transformation $g$ with $a=I_{2}$ and $\lambda=v=1$ shows that the condition $\tilde{\rho}_{i}=0$ is written as follows:

$$
b\left(c^{1}\right)^{2}+\ell_{11} c^{1}+\rho_{1}=0, \quad c\left(c^{2}\right)^{2}+\ell_{22} c^{2}+\rho_{2}=0 .
$$

Here $\left(c^{1}, c^{2}\right)$ is a vector appearing in a change of the frame in Proposition 2.1. The term $c^{2}$ here is not $(c)^{2}$.

Proposition 2.5. Assume that the surface is indefinite and non-ruled. Then, there is a frame so that $\rho_{i}=0$. The number of such frames is at most four.

Let us denote by $\Delta_{1}$ and $\Delta_{2}$ the discriminants of the equations of (2.19):

$$
\Delta_{1}=\left(\ell_{11}\right)^{2}-4 \rho_{1} b \text { and } \Delta_{2}=\left(\ell_{22}\right)^{2}-4 \rho_{2} c
$$

respectively. We remark that, when $\Delta_{1}$ and/or $\Delta_{2}$ are negative or when $h$ is definite, we need to make considerations in the complex number field.

Definition 2.6. We call such a frame a Demoulin frame.

Once a Demoulin frame is chosen, the remaining freedom of choice is very restricted. For simplicity consider the case $\lambda v=1$. Then the only possible form of the frame change $g$ is

$$
\left(\begin{array}{cccc}
\lambda & 0 & 0 & 0 \\
\lambda \alpha c^{2} & \alpha & 0 & 0 \\
\lambda \alpha^{-1} c^{1} & 0 & \alpha^{-1} & 0 \\
\lambda c^{1} c^{2} & c^{1} & c^{2} & \lambda^{-1}
\end{array}\right)
$$

and the values $c^{1}$ and $c^{2}$ must satisfy the conditions

$$
b\left(\lambda c^{1}\right)^{2}+\ell_{11} \lambda c^{1}=0, \quad c\left(\lambda c^{2}\right)^{2}+\ell_{22} \lambda c^{2}=0 .
$$

For each solution, the new $\tilde{\ell}_{i j}$ are given by

$$
\tilde{\ell}_{11}=\ell_{11}+2 b \lambda c^{1}, \quad \tilde{\ell}_{22}=\ell_{22}+2 c \lambda c^{2},
$$

and the last vector of the new frame is

$$
\lambda^{-1}\left(e_{3}+\left(\lambda c^{1}\right) e_{1}+\left(\lambda c^{2}\right) e_{2}+\left(\lambda^{2} c^{1} c^{2}\right) e_{0}\right) .
$$

Definition 2.7. When the vector $e_{3}$ of a Demoulin frame defines a surface, we call it a Demoulin transform of the original surface. 
For a Demoulin frame, we set

$$
\sum_{j} h_{i j} \omega_{3}^{j}=\sum_{j} q_{i j} \omega^{j}, \quad \omega_{i}^{0}=\sum_{j} p_{i j} \omega^{j} .
$$

(The letters $p$ and $q$ are reserved for denoting the coefficients of the system; the usage here for the matrices can be distinguished from the context.) The condition for $e_{3}$ to define a surface is that $\omega_{3}^{1}$ and $\omega_{3}^{2}$ are linearly independent, because $d e_{3}=$ $\omega_{3}^{3} e_{3}+\omega_{3}^{1} e_{1}+\omega_{3}^{2} e_{2}$. In other words,

$$
\operatorname{det} q \neq 0, \quad q=\left(q_{i j}\right) .
$$

Under this condition, a set $\bar{e}=\left(e_{3}, e_{1}, e_{2}, e_{0}\right)$ in this order defines a projective frame of $e_{3}$, and the coframe $\bar{\omega}$ is

$$
\bar{\omega}=\left(\begin{array}{cccc}
\omega_{3}^{3} & \omega_{3}^{1} & \omega_{3}^{2} & 0 \\
\omega_{1}^{3} & \omega_{1}^{1} & \omega_{1}^{2} & \omega_{1}^{0} \\
\omega_{2}^{3} & \omega_{2}^{1} & \omega_{2}^{2} & \omega_{2}^{0} \\
0 & \omega^{1} & \omega^{2} & \omega_{0}^{0}
\end{array}\right) .
$$

Therefore, the associated fundamental form $\bar{\varphi}_{2}$ is $\omega_{3}^{1} \cdot \omega_{1}^{0}+\omega_{3}^{2} \cdot \omega_{2}^{0}$, which is nondegenerate when

$$
\operatorname{det} p \neq 0, \quad p=\left(p_{i j}\right) \text {. }
$$

As $\ell_{i j}=q_{i j}-p_{i j}$ satisfies the condition $\ell=\operatorname{tr} \ell_{i j}=0$, we see that

$$
p_{12}=q_{12}, \quad p_{21}=q_{21},
$$

from which we have

$\bar{\varphi}_{2}=p_{21}\left(p_{11}+q_{11}\right) \omega^{1} \omega^{1}+\left(2 p_{12} p_{21}+p_{11} q_{22}+q_{11} p_{22}\right) \omega^{1} \omega^{2}+p_{12}\left(p_{22}+q_{22}\right) \omega^{2} \omega^{2}$.

Moreover, $\omega_{3}^{0}=0$ implies $\sum_{i} \omega_{3}^{i} \wedge \omega_{i}^{0}=0$. Hence,

$$
p_{11} q_{22}-p_{22} q_{11}=0 .
$$

Let us continue our consideration of the system (2.12) by assuming that $b c \neq 0$. By the expression in (2.15), a Demoulin frame is given by the change of the frame (2.14) by the transformation

$$
\left(\begin{array}{cccc}
1 & 0 & 0 & 0 \\
c^{2} & 1 & 0 & 0 \\
c^{1} & 0 & 1 & 0 \\
c^{1} c^{2} & c^{1} & c^{2} & 1
\end{array}\right)
$$


where

$$
c^{1}=\frac{-b_{y} \pm \sqrt{\Delta_{1}}}{2 b}, \quad c^{2}=\frac{-c_{x} \pm \sqrt{\Delta_{2}}}{2 c}
$$

and

$$
\Delta_{1}=\left(b_{y}\right)^{2}+4 b\left(b q+p_{y}-\frac{1}{2}(b c)_{x}\right), \quad \Delta_{2}=\left(c_{x}\right)^{2}+4 c\left(c p+q_{x}-\frac{1}{2}(b c)_{y}\right) .
$$

Hence, the Demoulin transforms $w$ of the surface $z$ are given by

$$
w=\left(c^{1} c^{2}-\frac{1}{2} b c\right) z+c^{1} z_{x}+c^{2} z_{y}+z_{x y} .
$$

An explicit computation of Demoulin frames is given in Section 11.6.

By the integrability (2.9), a simple computation shows

$$
\Delta_{1}=4 b^{2} Q, \quad \Delta_{2}=4 c^{2} P .
$$

By use of the Wilczynski frame $\left\{z, z_{1} . z_{2}, \eta\right\}$, the transform $w$ is given by

$$
w=\eta+\sigma z_{1}+\tau z_{2}+\sigma \tau z
$$

where $\sigma= \pm \sqrt{Q}$ and $\tau= \pm \sqrt{P}$.

Definition 2.8. We call a surface satisfying the conditions $b c \neq 0$ and $\operatorname{det} q \neq 0$ a Demoulin surface if it has only one Demoulin transform, equivalently if $P=Q=0$. A surface with $P=0$ or $Q=0$ is called a Godeaux-Rozet surface.

The condition $P=Q=0$ determines the coefficients $p$ and $q$ in terms of $b$ and $c$ by (2.18) and then the third equation of the integrability (2.13) turns out to be satisfied. The first and the second equations of the integrability (2.13) then give a system of nonlinear differential equations relative to $b$ and $c$ :

$$
\begin{gathered}
b^{2} b_{x y y}-b b_{x} b_{y y}-b b_{y} b_{x y}+b_{x} b_{y}{ }^{2}-2 c b^{3} b_{y}-b^{4} c_{y}=0, \\
c^{2} c_{x x y}-c c_{x} c_{x x}-c c_{x} c_{x y}+c_{x}{ }^{2} c_{y}-2 b c^{3} c_{x}-c^{4} b_{x}=0 .
\end{gathered}
$$

Moreover, this system can be integrated as follows:

$$
(\log b)_{x y}=b c+\frac{r(x)}{b}, \quad(\log c)_{x y}=b c+\frac{s(y)}{c}
$$

by using indeterminate functions $r$ of $x$ and $s$ of $y$. Hence, we have seen that a Demoulin surface is defined by the system

$$
z_{x x}=b z_{y}+\left(\frac{c_{x x}}{2 c}-\frac{c_{x}^{2}}{4 c^{2}}-\frac{b_{y}}{2}\right) z, \quad z_{y y}=c z_{x}+\left(\frac{b_{y y}}{2 b}-\frac{b_{y}^{2}}{4 b^{2}}-\frac{c_{x}}{2}\right) z,
$$

where $b$ and $c$ are satisfying the system (2.27). 
Remark 2.9. Relative to a Demoulin frame, any Godeaux-Rozet surface for which $b c \neq 0$ is characterized by the condition $\ell_{11}=0$ and $\ell_{22} \neq 0$ or $\ell_{11} \neq 0$ and $\ell_{22}=0$. Furthermore, in view of (2.23), we must have $p_{11}=q_{11}=0$ or $p_{22}=q_{22}=0$, respectively.

\subsection{Remarks on higher-dimensional hypersurfaces}

Let $\mathbf{A}^{n+1}$ be the $(n+1)$-dimensional affine space and $f_{0}: M^{n} \rightarrow \mathbf{A}^{n+1}$ an immersion defining an affine hypersurface. We choose a set of vectors $\left\{f_{1}, \ldots, f_{n+1}\right\}$ along the immersion so that $f_{1}, \ldots, f_{n}$ are tangent to the hypersurface and $\operatorname{det}\left(f_{1}, \ldots\right.$, $\left.f_{n+1}\right)=1$. Then we have a Pfaff equation $d f_{\alpha}=\sum_{\beta} \tau_{\alpha}^{\beta} f_{\beta}(0 \leq \alpha, \beta \leq n+1)$ and the connection form $\tau=\left(\tau_{\alpha}^{\beta}\right)$. The components of the form $\tau$ are

$$
\left(\begin{array}{ccc}
0 & \tau^{j} & \tau_{0}^{n+1} \\
0 & \tau_{i}^{j} & \tau_{i}^{n+1} \\
0 & \tau_{n+1}^{j} & \tau_{n+1}^{n+1}
\end{array}\right)
$$

The integrability condition $d \tau=\tau \wedge \tau$ is satisfied. Similarly to the argument of Section 2.1, we have $\tau_{0}^{n+1}=0$ and $\tau_{i}^{n+1}=\sum h_{i j} \tau^{j}$, where $\tau^{j}$ denotes $\tau_{0}^{j}$. We may assume that $|\operatorname{det} h|=1$ and $\tau_{n+1}^{n+1}=0$. Thus we get a quadratic form $\sum h_{i j} \tau^{i} \tau^{j}$. We define a tensor $m_{i j}$ by $\sum h_{i j} \tau_{n+1}^{j}=\sum m_{i j} \tau^{j}$. It is called the affine mean curvature tensor, and $m=\frac{1}{n} \sum h^{i j} m_{i j}$ is called the affine mean curvature. When we regard the affine hypersurface as a projective hypersurface, the invariant $h_{i j}$ remains the same, the invariant $\ell_{i j}$ is given by $\ell_{i j}=m_{i j}-m h_{i j}$, and the invariant form $\omega_{n+1}^{0}$ equals $-\frac{1}{2} d m$. It suffices to normalize the frame by a transformation

$$
\left(\begin{array}{ccc}
1 & 0 & 0 \\
0 & I_{n} & 0 \\
-\frac{1}{2} m & 0 & 1
\end{array}\right)
$$

When an affine hypersurface satisfies $m_{i j}-m h_{i j}=0$, it is called an affine hypersphere and then $m$ turns out to be constant. Namely, we have $\ell_{i j}=0$ and $\rho_{i}=0$ for affine hyperspheres. In the two-dimensional case, this property is equivalent to the definition of a Demoulin surface. We introduce the following definition.

Definition 2.10. A projective hypersurface is called a Demoulin hypersurface if it has a frame so that $\ell_{i j}=0$ and $\rho_{i}=0$. 
The property that $\ell_{i j}=0$ and $\rho_{i}=0$ for one frame is equivalent to the property that for any frame described in Proposition 2.1, there exists a vector $a^{k}$ such that

$$
\ell_{i j}=\sum h_{i j k} a^{k} \quad \text { and } \quad \rho_{i}=-\frac{1}{2} \sum h_{i j k} a^{j} a^{k}
$$

due to the invariance in Proposition 2.1.

We further consider whether there exists a frame so that $\rho_{i}=0$ for a general hypersurface. To find such a frame is equivalent to solving the equation

$$
\rho_{i}+\sum_{j} \ell_{i j} c^{j}-\frac{1}{2} \sum_{j, k} h_{i j k} c^{j} c^{k}=0 \quad(1 \leq i \leq n)
$$

relative to $c^{i}$; see Proposition 2.2. If the quadratic forms $H_{i}(c, c)=\sum h_{i j k} c^{j} c^{k}$ are in general position, then it is possible to solve the equations and the number of solutions is $2^{n}$. However, in our case the coefficients satisfy algebraic conditions

$$
\sum_{i, j} h^{i j} \ell_{i j}=0, \quad \sum_{i, j} h^{i j} h_{i j k}=0
$$

and also the conditions that the $\ell_{i j}$ and $h_{i j k}$ are totally symmetric. A computer test done by Noro for the case $n=3$ shows that the number of solutions is eight. Furthermore, when $n=3$, the identities $\sum h_{i j k} c^{j} c^{k}=0$ imply that $c^{1}=c^{2}=$ $c^{3}=0$, and especially the identities $\sum h_{i j k} c^{k}=0$ imply that $c^{1}=c^{2}=c^{3}=0$. This means that, for a Demoulin hypersurface, such a frame is unique. The author wonders if this also holds generically.

Remark 2.11. Equation (2.29) has the following geometrical meaning. Let us recall the definition of the Lie quadratic hypersurface $Q$ defined by the equation $E=0$ in Section 2.1. The set of hypersurfaces $Q$ is stationary at a point $P$ if and only if $d E=0$ also at $P$. As we have seen, the condition $d E=0$ is $p H_{i}{ }^{\mathrm{t}} p=0$ for each $i$ and this is written as

$$
\rho_{i}\left(p^{n+1}\right)^{2}+\sum_{j} \ell_{i j} p^{j} p^{n+1}-\frac{1}{2} \sum_{j, k} h_{i j k} p^{j} p^{k}=0 \quad(1 \leq i \leq n),
$$

which is a homogeneous form of equation (2.29). We refer to Sections 11.3 and 11.4 for the detailed study of the two-dimensional case.

\subsection{Projectively applicable surfaces}

Relative to the Wilczynski frame, the invariants $\kappa_{1}=\left(b c-(\log b)_{x y}\right) / 2$ and $\kappa_{2}=$ $\left(b c-(\log c)_{x y}\right) / 2$ have played important roles. In this section, we examine their 
geometric meaning. For this purpose, we consider a space curve $z(x, y)$ by fixing the variable $y$. Set $w(x)=b^{-1 / 2} z$. Then a calculation shows

$$
w_{x x x x}+q_{2} w_{x x}+q_{3} w_{x}+q_{4} w=0,
$$

where

$$
\begin{aligned}
& q_{2}=2(\log b)_{x x}-\left((\log b)_{x}\right)^{2} / 2-b_{y}-2 p, \\
& q_{3}=2(\log b)_{x x x}-(\log b)_{x}\left((\log b)_{x x}+b_{y}\right)-2 p_{x}-b^{2} c .
\end{aligned}
$$

Hence, the invariant form $\psi_{3}$ introduced in Section 1.1 is given by

$$
\psi_{3}=-b\left(b c-(\log b)_{x y}\right) d x^{3}=-2 b \kappa_{1} d x^{3} .
$$

We give here a lemma without proof.

LEMMA 2.12. Set

$$
\begin{aligned}
& K^{1}=\log \left(b \kappa_{1}\right)=\log \left(b\left(b c-(\log b)_{x y}\right) / 2\right), \\
& K^{2}=\log \left(c \kappa_{2}\right)=\log \left(c\left(b c-(\log c)_{x y}\right) / 2\right) .
\end{aligned}
$$

Then

$$
P_{y}=2 \kappa_{2} K_{x}^{2}, \quad Q_{x}=2 \kappa_{1} K_{y}^{1} .
$$

In particular, for a Demoulin surface with $\kappa_{1} \kappa_{2} \neq 0$,

$$
\left(\log c \kappa_{2}\right)_{x}=0, \quad\left(\log b \kappa_{1}\right)_{y}=0 .
$$

For a Godeaux-Rozet surface, one of $\left(c\left(b c-(\log c)_{x y}\right)\right)_{x}=0$ and $(b(b c-$ $\left.\left.(\log b)_{x y}\right)\right)_{y}=0$ will hold. When the former identity holds, we can set $c(b c-$ $\left.(\log c)_{x y}\right)=Y(y)$ and, when this does not vanish, we can assume $Y=1$ by a simple argument on the coordinate dependence. On the other hand, this says that the parameter $y$ is proportional to the projective length parameter. Hence, we have the following.

Proposition 2.13. For any Demoulin surface, the coordinates $(x, y)$ can be chosen so that $x$ and $y$ are projective length parameters of both coordinate curves.

From (2.30), we can also conclude the following proposition.

Proposition 2.14. Let $z$ be a non-ruled surface. Then:

(1) the space curve with parameter $x$ (respectively parameter $y$ ) belongs to a linear complex if and only if $\kappa_{1}=0$ (respectively $\left.\kappa_{2}=0\right)$; 
(2) if both curves belong to linear complexes, then the system reduces to the case

$$
b=c, \quad(\log b)_{x y}=b^{2} .
$$

Proof. Part (1) follows directly from Proposition 1.1. For part (2), we have

$$
b c=(\log b)_{x y}=(\log c)_{x y} .
$$

In particular, $(\log b / c)_{x y}=0$. Hence, we can write $b / c=X(x) Y(y)$ for nonvanishing functions $X$ and $Y$. As, by coordinate change from $(x, y)$ to $(\bar{x}=f(x), \bar{y}=$ $g(y))$ the invariants $b$ and $c$ are changed to $b g^{\prime} / f^{\prime 2}$ and $c f^{\prime} / g^{\prime 2}$, we can assume $b=c$ and the conclusion follows.

We next treat the surface with the property (2.32). First, note that

$$
L=-b_{y}-2 p, \quad M=-c_{x}-2 q .
$$

Differentiating (2.32), we get the identity

$$
\left((\log b)_{x x}+\frac{1}{2}(\log b)_{x}^{2}\right)_{y}=3 b b_{x}
$$

and we see that $L_{y}=-3 b b_{x}$, by (2.9). Hence, there exists a function $X_{1}(x)$ such that

$$
L=-(\log b)_{x x}-\frac{1}{2}(\log b)_{x}^{2}+X_{1}(x) .
$$

Similarly, we can set

$$
M=-(\log b)_{y y}-\frac{1}{2}(\log b)_{y}^{2}+Y_{1}(x) .
$$

Then, from the third formula of the integrability (2.9), it holds that

$$
\left(b^{2} Y_{1}\right)_{y}=\left(b^{2} X_{1}\right)_{x} .
$$

On the other hand, any solution of the equation $(\log b)_{x y}=b^{2}$ is written as

$$
b=\frac{\left(X^{\prime} Y^{\prime}\right)^{1 / 2}}{X+Y}, \quad \text { where } X=X(x), \quad Y=Y(y) .
$$

Hence, integrating the form $b^{2} Y_{1} d x+b^{2} X_{1} d y$, which is closed by (2.33), we get the identity

$$
-\frac{Y^{\prime} Y_{1}}{X+Y}+Y_{2}=-\frac{X^{\prime} X_{1}}{X+Y}+X_{2}
$$

for appropriate functions $X_{2}(x)$ and $Y_{2}(y)$. By differentiation relative to $x$ and $y$, we get

$$
Y_{2}^{\prime} X^{\prime}=Y^{\prime} X_{2}^{\prime}
$$


This implies that there exist constants $k_{1}, k_{2}$, and $k_{3}$ so that

$$
X_{2}=k_{1} X+k_{2}, \quad Y_{2}=k_{1} Y+k_{3} .
$$

Then, we see that

$$
X^{\prime} X_{1}-k_{1} X^{2}-\left(k_{2}-k_{3}\right) X=Y^{\prime} Y_{1}-k_{1} Y^{2}-\left(k_{3}-k_{2}\right) Y,
$$

which must be constant, and let us call this constant $c_{3}$. By setting $k_{2}-k_{3}=c_{2}$ and $k_{1}=c_{1}$, we finally get

$$
X^{\prime} X_{1}=c_{1} X^{2}+c_{2} X+c_{3}, \quad Y^{\prime} Y_{1}=c_{1} Y^{2}-c_{2} Y+c_{3} .
$$

Now we have explicit forms of $p$ and $q$ in terms of two functions $X$ and $Y$ and three constants:

$$
\begin{aligned}
& 2 p=-b_{y}+(\log b)_{x x}+\frac{1}{2}(\log b)_{x}^{2}-\frac{c_{1} X^{2}+c_{2} X+c_{3}}{X^{\prime}}, \\
& 2 q=-b_{x}+(\log b)_{y y}+\frac{1}{2}(\log b)_{y}^{2}-\frac{c_{1} Y^{2}-c_{2} Y+c_{3}}{Y^{\prime}} .
\end{aligned}
$$

This fact says that, for a surface with the property (2.32), the possible choices for $p$ and $q$ can include three arbitrary constants. As this is a remarkable property, we recall the following definition.

Definition 2.15. Given two surfaces $S_{1}$ and $S_{2}$, a mapping $f: S_{1} \rightarrow S_{2}$ is called a projective deformation if, for any point $p$ in $S_{1}$, there exists a projective transformation $T$ such that $T(p)=f(p)$ and that, for any curve $C$ on $S_{1}$ passing through $p$, its image $T(C)$ has a second-order contact with the curve $f(C)$. A surface is said to be projectively applicable if it has a non-trivial projective deformation.

Proposition 2.16. If $f: S_{1} \rightarrow S_{2}$ is a projective deformation, then the asymptotic directions are preserved by $f$. Also, $b_{1}=b_{2}$ and $c_{1}=c_{2}$ when denoting the invariants for $S_{1}$ by $b_{1}$ and $c_{1}$ and for $S_{2}$ by $b_{2}$ and $c_{2}$, relative to the common asymptotic directions. These conditions are conversely sufficient for the two surfaces to be related by a projective deformation.

Proof. From the second-order contactedness, it immediately follows that the asymptotic directions are preserved. Hence, both surfaces can be assumed to be given by systems such as (2.12): assume that $S_{1}$ is given by the system $\left(z_{1}\right)_{x x}=$ $b_{1}\left(z_{1}\right)_{y}+p_{1} z_{1}$ and $\left(z_{1}\right)_{y y}=c_{1}\left(z_{1}\right)_{x}+q_{1} z_{1}$ and that $S_{2}$ is given by the system $\left(z_{2}\right)_{x x}=b_{2}\left(z_{2}\right)_{y}+p_{2} z_{2}$ and $\left(z_{2}\right)_{y y}=c_{2}\left(z_{2}\right)_{x}+q_{2} z_{2}$. Up to a projective transformation, we have $z_{2}=\rho z_{1},\left(z_{2}\right)_{x}=\rho_{x} z_{1}+\rho z_{1 x},\left(z_{2}\right)_{y}=\rho_{y} z_{1}+\rho z_{1}$, 
and $\left(z_{2}\right)_{x x}=\rho_{x x} z_{1}+2 \rho_{x}\left(z_{1}\right)_{x}+\rho\left(z_{1}\right)_{x x}$ at the contact point. Hence, we can see that $\left(z_{2}\right)_{x x}=b_{1}\left(z_{2}\right)_{y}+2 \rho_{x} / \rho\left(z_{2}\right)_{x}+\left(p_{1}+\rho_{x x} / \rho-b_{1} \rho_{x} / \rho-2 \rho_{x}^{2} / \rho^{2}\right) z_{2}$, which implies that $b_{1}=b_{2}$. Similarly, $c_{1}=c_{2}$. The converse statement is seen by reversing the argument, as derivatives of $\rho$ can take arbitrary values at the contact point.

Any (non-degenerate) ruled surface is projectively applicable, because the coefficient $p$ includes an arbitrary function $\delta$ in the representation (2.16).

Next we assume $b c \neq 0$ and consider a surface given by the system (2.12). We want to find distinct surfaces with the same $b$ and $c$. Let $L_{1}, M_{1}$ and $L_{2}, M_{2}$ be the quantities for two surfaces that are deformable to each other. By setting

$$
\lambda=L_{2}-L_{1}, \quad \mu=M_{2}-M_{1}
$$

and checking the integrability, we get the conditions

$$
\lambda_{y}=\mu_{x}=0, \quad b \mu_{y}+2 \mu b_{y}=c \lambda_{x}+2 \lambda c_{x} .
$$

Then, introducing $v$ by

$$
v b c=b \mu_{y}+2 \mu b_{y},
$$

we get the compatibility condition

$$
2 \lambda(\log c)_{x y}=(v b)_{y}, \quad 2 \mu(\log b)_{x y}=(\nu c)_{x} .
$$

If the system of the linear differential equations (2.36) and (2.37) relative to $\lambda$, $\mu$, and $v$ is solvable, then we have at most three independent solutions. We denote them by $\left(\lambda_{i}, \mu_{i}\right)$ for $i \leq 3$. General solutions are written as $\lambda=\sum c_{i} \lambda_{i}$ and $\mu=\sum c_{i} \mu_{i}$, where $c_{i}$ are integration constants.

PROPOSITION 2.17. Assume that $b c \neq 0$ and the number of independent solutions is three. Then the Gaussian curvature of the form $b c d x d y$ is constant.

Proof. We compute $v_{x y}$ from (2.37) in two different orders $\left(v_{x}\right)_{y}$ and $\left(v_{y}\right)_{x}$ and get the identity

$$
2 c \lambda\left((\log c)_{x y} / b c\right)_{x}-2 b \mu\left((\log b)_{x y} / b c\right)_{y}=v(\log b / c)_{x y} .
$$

As we have assumed the existence of three independent solutions of the triple $(\lambda, \mu, v)$, this equation should be trivial. Namely, we have

$$
(\log b / c)_{x y}=0, \quad\left((\log c)_{x y} / b c\right)_{x}=0, \quad\left((\log b)_{x y} / b c\right)_{y}=0 .
$$

The latter two equations show that $(\log b c)_{x y} / b c$ is constant, as $(\log b)_{x y}=(\log c)_{x y}$, namely, the Gaussian curvature of the metric form $b c d x d y$ is constant. 
Remark 2.18. The first relation of (2.38) shows that we can assume $b=c$ by a suitable coordinate change. A surface with the condition $b=c$ was called an isothermicasymptotic surface by Fubini.

Example 2.19. The system

$$
z_{x x}=z_{y}+\left(k x+k_{1}\right) z, \quad z_{y y}=z_{x}+\left(k x+k_{2}\right),
$$

where $k, k_{1}$ and $k_{2}$ are constants, is integrable. Any surface defined by this system is called a coincidence surface. When these constants are all zero, the surface is equivalent to $X Y Z=1$ in affine space. By Proposition 2.16, any coincidence surface is projectively applicable to the surface $X Y Z=1$.

Example 2.20. The system called the Appell's system $\left(F_{4}\right)$ is the following:

$$
\begin{aligned}
z_{x x}= & \frac{2 y}{1-x-y} z_{x y}+\frac{(a+b+1) x-c_{1}(1-y)}{x(1-x-y)} z_{x} \\
& +\frac{\left(a+b+1-c_{2}\right) y}{x(1-x-y)} z_{y}+\frac{a b}{x(1-x-y)} z \\
z_{y y}= & \frac{2 x}{1-x-y} z_{x y}+\frac{\left(a+b+1-c_{1}\right) x}{y(1-x-y)} z_{x}+\frac{(a+b+1) y-c_{2}(1-x)}{y(1-x-y)} z_{y} \\
& +\frac{a b}{y(1-x-y)} z,
\end{aligned}
$$

where $a, b, c_{1}$ and $c_{2}$ are complex constants. An asymptotic coordinate system $(u, v)$ is defined by the coordinate change $(x, y)=(u(1-v), v(1-u))$. Relative to the new unknown function $w=(u(1-v))^{c_{1}}(v(1-u))^{1-c_{2}}(1-u-v)^{-e} z$ where $e=c_{1}+c_{2}-a-b-1$, the system is changed to

$$
\begin{aligned}
& z_{u u}=-\frac{e v(1-v)}{u(1-u)(1-u-v)} z_{v}+p z, \\
& z_{v v}=-\frac{e u(1-u)}{v(1-v)(1-u-v)} z_{u}+q z,
\end{aligned}
$$

where

$$
\begin{aligned}
& p=p_{0}+\frac{a_{1}}{u(1-u)}+\frac{a_{2}}{u^{2}}+\frac{a_{3}}{(1-u)^{2}}, \\
& q=q_{0}+\frac{a_{1}}{v(1-v)}+\frac{a_{3}}{v^{2}}+\frac{a_{2}}{(1-v)^{2}},
\end{aligned}
$$




$$
\begin{aligned}
p_{0}= & \frac{e}{2(1-u-v)^{2}} \\
& +\frac{e^{2}}{4}\left(\frac{2}{u(1-v)}+\frac{2}{v(1-u)}-\frac{2(1-2 v)}{v(1-v)(1-u-v)}+\frac{3}{(1-u-v)^{2}}\right), \\
q_{0}= & \frac{e}{2(1-u-v)^{2}} \\
& +\frac{e^{2}}{4}\left(\frac{2}{u(1-v)}+\frac{2}{v(1-u)}-\frac{2(1-2 u)}{u(1-u)(1-u-v)}+\frac{3}{(1-u-v)^{2}}\right),
\end{aligned}
$$

and

$a_{1}=\left(2 a b-c_{1} c_{2}+(a+b+1) e\right) / 2, \quad a_{2}=c_{1}\left(-2+c_{1}\right) / 4, \quad a_{3}=c_{2}\left(-2+c_{2}\right) / 4$.

This shows that the system above defines projectively applicable surfaces with three parameters for each constant $e=c_{1}+c_{2}-a-b-1$. The Gaussian curvature referred to in Proposition 2.17 is equal to $-2 / e^{2}$.

Now we go back to the general situation. It is easy to see that $\lambda$ and $\mu$ defined in (2.35) change to $\lambda / f^{\prime 2}$ and $\mu / g^{\prime 2}$ by the coordinate change from $(x, y)$ to $(\bar{x}=f(x), \bar{y}=g(y))$. Therefore, when $\lambda \mu \neq 0$, we can reduce the system to one where $\lambda=\mu=1$ and, when $\lambda \neq 0$ and $\mu=0$, to a system where $\lambda=1$ and $\mu=0$. The corresponding conditions on $b$ and $c$ are either $c_{x}=b_{y}$ or $c_{x}=0$.

Definition 2.21. The surface with $c_{x}=b_{y}$ is called an $R$-surface and the surface with $c_{x}=0$ is called an $R_{0}$-surface.

For an $R$-surface, $\lambda=\mu$ can be any constant. Namely, the integrability condition holds for $p+k$ and $q+k$ for any constant $k$ with the same cubic invariants $b$ and $c$. For an $R_{0}$-surface, $\mu=0$ and $\lambda$ can be any constant. Hence, the integrability condition holds for $p+k$ and $q$ with the same cubic invariants for any constant $k$.

\subsection{Projectively minimal surfaces}

In Section 2.2 we saw that the form $F \varphi_{2}$ is well-defined independent of the frames. When $F \neq 0, F \varphi_{2}$ defines an area functional and any critical surface relative to this functional is called a projectively minimal surface.

It is known that, relative to the system (2.12), the condition for projective minimality is written as

$$
b M_{y}+2 M b_{y}+b_{y y y}=0, \quad c L_{x}+2 L c_{x}+c_{x x x}=0 .
$$


By the integrability (2.9), each of the above equations is equivalent to the other. In terms of the invariants $P$ and $Q$, the equation is rewritten as

$$
b Q_{y}+2 b_{y} Q=0, \quad c P_{x}+2 c_{x} P=0
$$

and, equivalently by (2.24),

$$
\left(\Delta_{1}\right)_{y}=0, \quad\left(\Delta_{2}\right)_{x}=0 .
$$

We assume that the surface is of indefinite type and non-ruled, and let $e$ be a Demoulin frame. Then the condition (2.41) is seen to be equivalent to

$$
\operatorname{det} p=\operatorname{det} q,
$$

where the matrices $p$ and $q$ are as defined in (2.20). For a proof we need explicit formulas of $p$ and $q$ in terms of the coefficients of the system, which will be given in (11.13). For the notation used in this formula, we refer to Section 11.3. Furthermore, by the identities (2.21) and (2.23), the condition in (2.42) is equivalent to each one of

$$
\text { (1) } \ell_{22}\left(p_{11}+q_{11}\right)=0 \text { and (2) } \ell_{11}\left(p_{22}+q_{22}\right)=0 \text {. }
$$

More generally, we can state the following proposition.

Proposition 2.22. A non-degenerate surface is projectively minimal if and only if

$$
\sum_{i, j} \ell^{i j}\left(p_{i j}+q_{i j}\right)=0, \quad \text { where } \ell^{i j}:=\sum_{k, m} h^{i k} h^{j m} \ell_{k m},
$$

for a Demoulin frame.

The computation of the condition (2.39) was given in [Th26]. The differential equation of projective minimality in general dimension was given in [Sa87] in terms of affine invariants and by use of the formulation in [Sa87] the above proposition was proved in [Sa99]. Equivalence of (2.41) and (2.42) follow more directly from (11.8), (11.11), (11.12) and (11.13) by using an explicit form of a Demoulin frame. The next theorem states a fundamental property of projectively minimal surfaces relative to Demoulin transform.

THEOREM 2.23. Let $S$ be a non-degenerate surface of indefinite type. Assume that the conditions $b c \neq 0, \ell_{11} \ell_{22} \neq 0$ and $\operatorname{det} p \neq 0$ for a Demoulin frame. Then:

(1) if $S$ is projectively minimal, then the conformal structure of a Demoulin transform of $S$ is the same as the conformal structure of $S$; 
(2) conversely, if the conformal structure of a Demoulin transform is the same as the conformal structure of the original surface, then the original surface is projectively minimal or a surface with the property $p_{12} p_{21}=0$.

Proof. As $\ell_{11} \ell_{22} \neq 0$ by assumption, the conditions in (2.43) show that $\bar{\varphi}_{2}$ is conformal to $\varphi_{2}=\omega^{1} \omega^{2}$ in (2.22). The converse is also immediate.

Remark 2.24. A correspondence that preserves conformal structures in the theorem defines a $W$-congruence in the terminology explained in Section 3.2. The surface with $p_{12} p_{21}=0$ is called a $Q$-surface in [Bol, vol. 2, p. 318].

Example 2.25. By Definition 2.8 and (2.40) or by (2.43), a Godeaux-Rozet surface is projectively minimal. In particular, a Demoulin surface is also projectively minimal.

Remark 2.26. The books [FC1, FC2, L42] as well as [AG93, Bol, Ka33] are good references to the theory of projective surfaces. We refer the reader to [Sa88, Sa99] for further details regarding Sections 2.1-2.5, and to [L42] for Section 2.2. The Wilczynski frame is given in [W07]. A generalization of the subject in Section 2.1 is given in [Se88, MSY93, SYY97]. The proof of Proposition 2.22 is given in [Sa99]. We also refer the reader to [Th26, May32]. The notations $L$ and $M$ were introduced in [FC2]. See [Sa01] for further details on Example 2.20. For projective applicability and its further development, we refer the reader to [FC2, Ch. 6] and [Ca20]. For the related notions such as projective connection, we refer to [Ca37]. Kanitani [Ka22] classified projectively applicable surfaces with three parameters by determining the corresponding systems. Example 2.20 gives an explicit representation of such systems for the non-flat case. We also refer to Fubini [FC1, Section 69]. Demoulin surfaces were introduced by Demoulin [Dem24]. For a general theory of $R$-surfaces and $R_{0}$-surfaces, we refer to [FC2] and [Fe00a]; the latter serves as a good introduction to the theory of projective surfaces in view of integrable systems. We also refer the reader to [FS99].

\section{Line congruences I}

Line congruences and Laplace transformations are central notions treated in this article. In this section, we introduce elementary terminology on line congruences and explain what a W-congruence is, and in the next section we give fundamental properties of Laplace transformations. 


\subsection{Line congruences}

We call a two-parameter family of lines in three-dimensional projective space $\mathbb{P}^{3}$ a line congruence. Given a surface in three-dimensional Euclidean space, the family of normal lines to the surface is a typical example of a line congruence and is called the normal congruence. Its study was begun by Kummer [Ku60]. We give a sketch of an elementary treatment of normal congruences in Appendix B.

Another method of constructing a line congruence is given as follows. Given a family of curves on a surface such that through any point passes a curve belonging to this family, the set of tangent lines to the curves form a family of lines parametrized by points of the surface. This congruence is called the tangent congruence associated to the family of curves.

A practical way of presenting a line congruence is to give a pair of surfaces $\{z(x, y), w(x, y)\}$ with surface parameters $x$ and $y$. Then to these parameters we associate the line $\overline{z w}$ joining the two points $z(x, y)$ and $w(x, y)$, and thus obtain a line congruence. We denote it simply by $\{z, w\}$.

Let $t \mapsto(x(t), y(t))$ be a curve in the parameter space $(x, y)$. Then we get a ruled surface $\{z(x(t), y(t)), w(x(t), y(t))\}$. The condition for this surface to be developable is

$$
z \wedge w \wedge \frac{d}{d t} z \wedge \frac{d}{d t} w=0
$$

which is equivalent to

$$
P\left(\frac{d x}{d t}\right)^{2}+2 Q \frac{d x}{d t} \frac{d y}{d t}+R\left(\frac{d y}{d t}\right)^{2}=0,
$$

where

$$
\begin{gathered}
P=z \wedge w \wedge z_{x} \wedge w_{x} \\
2 Q=z \wedge w \wedge z_{x} \wedge w_{y}+z \wedge w \wedge z_{y} \wedge w_{x} \\
R=z \wedge w \wedge z_{y} \wedge w_{y}
\end{gathered}
$$

Hence, generally, there exist two directions on the parameter space so that, along the integral curves of these direction fields, the ruled surface is developable and its directrix curves for each family of directions comprise a surface; this surface is called the focal surface of the line congruence. We remark that the integral curves for normal congruence of a surface in Euclidean space are nothing but the curvature lines. We refer the reader to Appendix B. 
Let $\partial / \partial x$ be one of the degenerate directions, i.e. let us assume that $P=0$, and let $z$ be the corresponding focal surface. We then have

$$
z_{x} \equiv 0 \quad(\bmod z, w) .
$$

The tangent plane of $z$ is generated by $\left\{z, w, z_{y}\right\}$. If $w_{x} \equiv 0(\bmod z, w)$ throughout, then $(z \wedge w)_{x}=\lambda z \wedge w$; namely, the line $z \wedge w$ is stationary in the direction $\partial / \partial x$. To exclude this degenerate case, we assume now that $w_{x} \not \equiv 0(\bmod z, w)$.

We next let $\partial / \partial y$ be the other degenerate direction, i.e. $R=0$, and let $w$ be the focal surface. We see that

$$
w_{y} \equiv 0 \quad(\bmod z, w)
$$

and the tangent plane is generated by $\left\{z, w, w_{x}\right\}$. Here we need the condition $Q \neq 0$ :

$$
z \wedge w \wedge z_{y} \wedge w_{x} \neq 0
$$

which we assume in the following.

\subsection{W-congruences}

We write (3.1) and (3.2) more explicitly as

$$
\begin{aligned}
& z_{x}=m w+r z, \\
& w_{y}=s w+n z,
\end{aligned}
$$

where we assume $m \neq 0$ and $n \neq 0$ so that $z$ and $w$ really do depend on $x$ and $y$, respectively. Furthermore, we set

$$
\begin{array}{ll}
z_{y y} \equiv p w_{x} & \left(\bmod z, w, z_{y}\right), \\
w_{x x} \equiv q z_{y} & \left(\bmod z, w, w_{x}\right) .
\end{array}
$$

Then we have

$$
\begin{aligned}
z_{x x} & \equiv m w_{x} \quad(\bmod z, w), \\
z_{x y} & \equiv 0 \quad\left(\bmod z, w, z_{y}\right), \\
w_{x y} & \equiv 0 \quad\left(\bmod z, w, w_{x}\right), \\
w_{y y} & \equiv n z_{y} \quad(\bmod z, w) .
\end{aligned}
$$

We now compute the induced conformal structures on the surfaces. Set

$$
\begin{gathered}
E=z \wedge z_{x} \wedge z_{y} \wedge z_{x x}=m^{2} z \wedge w \wedge z_{y} \wedge w_{x}, \\
F=z \wedge z_{x} \wedge z_{y} \wedge z_{x y}=0, \\
G=z \wedge z_{x} \wedge z_{y} \wedge z_{y y}=m p z \wedge w \wedge z_{y} \wedge w_{x},
\end{gathered}
$$


then $E d x^{2}+2 F d x d y+G d y^{2}$ defines the conformal structure on the surface $z$, which turns out to be

$$
\varphi_{z}=m d x^{2}+p d y^{2}
$$

Similarly, we get

$$
\varphi_{w}=q d x^{2}+n d y^{2}
$$

which defines the conformal structure on the surface $w$. For the surfaces to be nondegenerate, the condition

$$
m n p q \neq 0
$$

is necessary and sufficient.

Definition 3.1. We say that the line congruence $\{z, w\}$ is a $W$-congruence ( $W$ is after Weingarten) if the conformal class of $\varphi_{z}$ is equal to the conformal class of $\varphi_{w}$. Namely, if it holds that

$$
W:=m n-p q=0 .
$$

Remark 3.2. If we say that two vectors $X$ and $Y$ are conjugate relative to a nondegenerate 2 -form $\varphi$ when $\varphi(X, Y)=0$, then a $\mathrm{W}$-congruence is a line congruence that preserves conjugate directions relative to $\varphi_{z}$ and $\varphi_{w}$. We refer to Section 4.1 for an intrinsic definition of a conjugate system.

Given a line congruence $\{z, w\}$, we define a surface $\xi$ lying in $\mathbb{P}^{5}$ by

$$
\xi=z \wedge w
$$

which lies in the quadratic hypersurface of $\mathbb{P}^{5}$ defined by the Plücker relation.

THEOREM 3.3. (Darboux) A congruence is a W-congruence if and only if the second osculating space of the surface $\xi$ is of codimension greater than one.

Proof. The second osculating space of $\xi$ is a space spanned by the derivatives of $\xi$ up to second order. It is enough to show that there exists a linear relation among the six vectors $\xi, \xi_{x}, \xi_{y}, \xi_{x x}, \xi_{x y}$ and $\xi_{y y}$ if and only if $W=0$. By a computation, we see that

$$
\begin{gathered}
\xi_{x}=z \wedge w_{x} \quad(\bmod \xi), \\
\xi_{y}=z_{y} \wedge w \quad(\bmod \xi), \\
\xi_{x x}=m w \wedge w_{x}+q z \wedge z_{y} \quad\left(\bmod \xi, \xi_{x}\right), \\
\xi_{x y}=z_{y} \wedge w_{x} \quad\left(\bmod \xi, \xi_{x}, \xi_{y}\right), \\
\xi_{y y}=p w_{x} \wedge w+n z_{y} \wedge z \quad\left(\bmod \xi, \xi_{y}\right) .
\end{gathered}
$$


Hence, for a linear relation such as

$$
A \xi_{x x}+2 B \xi_{x y}+C \xi_{y y} \equiv 0 \quad\left(\bmod \xi, \xi_{x}, \xi_{y}\right)
$$

to exist, it is enough to solve

$(A q-C n) z \wedge z_{y}+2 B z_{y} \wedge w_{x}+(A m-C p) w \wedge w_{x} \equiv 0 \quad\left(\bmod z \wedge w, z \wedge w_{x}, z_{y} \wedge w\right)$

As $z \wedge w \wedge z_{y} \wedge w_{x} \neq 0$ by the assumption (3.3), this identity is equivalent to

$$
A q-C n=0, \quad B=0, \quad A m-C p=0 .
$$

Therefore, $W=0$ is necessary for non-trivial $A$ and $C$ to exist, and vice versa.

\section{The Laplace transformation}

This section treats the Laplace transformation of the differential equation

$$
z_{x y}+a z_{x}+b z_{y}+c z=0
$$

which sends any solution of this equation to a solution of another differential equation of the same form. We first interpret it in terms of tangent congruence when $z$ is regarded as an immersion of a surface, and then give fundamental notions relative to the Laplace transformation following Darboux [D2].

\subsection{Laplace invariants}

Let $S$ be a surface in $\mathbb{P}^{3}$ defined by an immersion $z(x, y)$ with parameter $\{x, y\}$ and the tangent planes $T_{(x, y)}$.

Definition 4.1. The coordinate system $\{x, y\}$ is called a conjugate system if the limit of the line $T_{(x, y)} \cap T_{(x+d x, y)}$, as $d x \rightarrow 0$, tends to a line that is parallel to the vector $z_{y}$, or equivalently, as seen below, if the limit of the line $T_{(x, y)} \cap T_{(x, y+d y)}$, as $d y \rightarrow 0$, tends to a line that is parallel to the vector $z_{x}$.

We can interpret this definition in terms of $z$ as follows. Let $Z$ denote the coordinate vector of $\mathbb{P}^{3}$. Then, for each $(x, y)$, there exists a vector $A(x, y)$ in the dual space such that the tangent plane is written as

$$
T_{(x, y)}: \quad\langle A(x, y), Z\rangle=0,
$$

where $\langle\cdot, \cdot\rangle$ denotes the dual pairing, and $z$ satisfies

$$
\langle A, z\rangle=\left\langle A, z_{x}\right\rangle=\left\langle A, z_{y}\right\rangle=0 .
$$


As

$$
T_{(x+d x, y)}:\left\langle A(x, y)+A_{x}(x, y) d x, Z\right\rangle=0,
$$

the limit of the intersection of these planes is defined by the equation

$$
\langle A, Z\rangle=\left\langle A_{x}, Z\right\rangle=0 .
$$

Hence, the condition of the coordinates being conjugate is

$$
\left\langle A, z_{y}\right\rangle=\left\langle A_{x}, z_{y}\right\rangle=0 .
$$

Therefore, we also have

$$
0=\left\langle A_{x}, z_{y}\right\rangle=\left\langle A, z_{y}\right\rangle_{x}-\left\langle A, z_{x y}\right\rangle=-\left\langle A, z_{x y}\right\rangle .
$$

Hence, we see that the four vectors $z, z_{x}, z_{y}$, and $z_{x y}$ are linearly dependent. Also, because the surface is regular, the first three are linearly independent. This implies an equation of the form

$$
z_{x y}+a z_{x}+b z_{y}+c z=0
$$

holds. Conversely, this differential equation characterizes conjugate systems.

However, the derivation of equation $(E)$ is not unique. This is because the system of homogeneous coordinates is determined only up to a scalar multiple and, in the definition above, only the directions of the coordinates are considered. Namely, we have the following freedom:

(1) we can choose $w=\lambda^{-1} z$ in place of $z$, where $\lambda$ is a scalar function;

(2) we may choose coordinates $(s, t)$, for which $s=s(x)$ and $t=t(y)$.

Although exchange of $x$ and $y$ is also possible, for the sake of simplicity we do not consider it. Relative to the freedom above, equation $(E)$ is changed as follows:

$$
w_{x y}+\left(a+(\log \lambda)_{y}\right) w_{x}+\left(b+(\log \lambda)_{x}\right) w_{y}
$$

$$
\begin{aligned}
& +\left(c+a(\log \lambda)_{x}+b(\log \lambda)_{y}+\lambda_{x y} / \lambda\right) w=0 \\
& z_{s t}+a \frac{d y}{d t} z_{s}+b \frac{d x}{d s} z_{t}+c \frac{d x}{d s} \frac{d y}{d t} z=0 .
\end{aligned}
$$

Now let us set

$$
h=a b+a_{x}-c, \quad k=a b+b_{y}-c .
$$

Then, if we denote the $h$ and $k$ for the new equations by $h^{\prime}$ and $k^{\prime}$, we have the identities

$$
h=h^{\prime}, \quad k=k^{\prime}
$$

for (1) and

$$
h d x d y=h^{\prime} d s d t, \quad k d x d y=k^{\prime} d s d t
$$

for (2). Hence, we have the following. 
Proposition 4.2. The 2-forms $h d x d y$ and $k d x d y$ are invariantly defined for the conjugate system.

Definition 4.3. We call these two 2-forms ( $h$ and $k$ for short) the Laplace invariants.

\section{Remark 4.4.}

(i) According to (1), the quantity $a b-c$ is changed into $a b-c-(\log \lambda)_{x y}$. Hence, we can always reduce the equation to the form $a b=c$ by choosing $\lambda$ appropriately. In this case, the invariants are $h=a_{x}$ and $k=b_{y}$.

(ii) If $h=k$, then $a_{x}=b_{y}$. Hence,

$$
a+(\log \lambda)_{y}=0, \quad b+(\log \lambda)_{x}=0
$$

is solvable. This implies that equation $(E)$ can be reduced to

$$
z_{x y}+c z=0 .
$$

(iii) If we choose $\lambda$ so that $a+(\log \lambda)_{y}=0$, then the new $c$, which is equal to $c+a(\log \lambda)_{x}+b(\log \lambda)_{y}+\lambda x y / \lambda$, turns out to be $h$ itself. Hence, if $h=0$, then the equation is reduced to a simpler equation of the form

$$
z_{x y}+b z_{y}=0 \text {. }
$$

Furthermore, if $h=0$, then equation $(E)$ is solvable as follows. As equation $(E)$ is written as $\left(z_{y}+a z\right)_{x}=-b\left(z_{y}+a z\right)$ and the equation $z_{x}^{1}=-b z^{1}$ is solved by $z^{1}=Y(y) e^{\int b d x}$ where $Y(y)$ is a space curve, $z$ has the form

$$
z=e^{-\int a d y}\left\{X(x)+\int^{y} Y(t) e^{\int a d y-\int b d x} d t\right\},
$$

where $X$ is an arbitrary space curve.

\subsection{The Laplace transformation}

We define the Laplace transformation in terms of tangent congruence. Let $z$ be a surface with a conjugate system of coordinates $\{x, y\}$. We choose a point $w$ on each tangent line in the direction $y$; it is written as

$$
w=z_{y}+\lambda z
$$

with parameter $\lambda$. We assume that the point $w$ draws a surface and that the line $\overline{z w}$ is again tangent to the surface $w$; this implies that, relative to the congruence $\{z, w\}$, the two surfaces $z$ and $w$ are focal surfaces. As

$$
w_{x}=(\lambda-a) z_{x}-b z_{y}+\left(\lambda_{x}-c\right) z,
$$


this assumption is satisfied if

$$
\lambda=a \text {. }
$$

Thus, we can introduce the following definition.

Definition 4.5. The surface $z^{1}=z_{y}+a z$ is called the first Laplace transform of $z$ and, similarly, the surface $z^{-1}=z_{x}+b z$ is called the minus-first Laplace transform.

We give some remarks on the definition. From equation $(E)$ we see that

$$
z_{x}^{1}=-b z^{1}+h z
$$

where $h$ is one of the Laplace invariants, and

$$
z_{x y}^{1}+a_{1} z_{x}^{1}+b_{1} z_{y}^{1}+c_{1} z^{1}=0
$$

where

$$
\begin{gathered}
a_{1}=a-(\log h)_{y}, \\
b_{1}=b, \\
c_{1}=c-a_{x}+b_{y}-b(\log h)_{y} .
\end{gathered}
$$

Hence,

$$
\left(z^{1}\right)^{-1}=h z
$$

and, if $h \neq 0$, the surface $\left(z^{1}\right)^{-1}$ is the same as the surface $z$. Similarly, if $k \neq 0$, the surface $\left(z^{-1}\right)^{1}$ is the same as $z$.

Let us next examine the case when $z^{1}$ is degenerate. This is the case where

$$
A z_{x}^{1}+B z_{y}^{1}+C z^{1}=0
$$

for certain scalar functions $A, B$, and $C$. If $B$ is identically zero, then we have $A\left(-b z^{1}+h z\right)+C z^{1}=0$, which holds only if $h=0$. If $B$ is not vanishing, then $\left(z^{1}\right)_{y} \equiv 0\left(\bmod z^{1},\left(z^{1}\right)_{x}\right)$; hence, $\left(z^{1}\right)_{y} \equiv 0\left(\bmod z, z_{y}\right)$. As $z_{y}^{1}=z_{y y}+(a z)_{y}$, we have $z_{y y} \equiv 0\left(\bmod z, z_{y}\right)$. This means that $z$ is ruled. If we further have $h \neq 0$, then $z^{1}$ is a curve and the ruling lines are tangent to this curve, hence the surface $z$ is developable.

Proposition 4.6. The transform $z^{1}$ is degenerate if and only if $h=0$ or if the surface $z$ is developable.

Remark 4.7. The osculating plane of $z^{1}$ along the $x$-curve is $z^{1} \wedge z_{x}^{1} \wedge z_{x x}^{1}$. As

$$
\begin{gathered}
z_{x}^{1}=-b z_{y}+\left(a_{x}-c\right) z, \\
z_{x x}^{1}=h z_{x}+\left(b^{2}-b_{x}\right) z_{y}+\left(b c+a_{x x}-c_{x}\right) z,
\end{gathered}
$$


we have

$$
z^{1} \wedge z_{x}^{1} \wedge z_{x x}^{1}=h^{2} z \wedge z_{x} \wedge z_{y}
$$

i.e. the osculating plane coincides with the tangent plane of $z$, unless $h=0$.

Example 4.8. Consider the equation

$$
(x-y) \frac{\partial^{2} z}{\partial x \partial y}+n \frac{\partial z}{\partial x}-m \frac{\partial z}{\partial y}=0,
$$

where $m$ and $n$ are constants. A surface satisfying this equation is, for example, given by

$$
z=\left[(x-a)^{m}(y-a)^{n},(x-b)^{m}(y-b)^{n},(x-c)^{m}(y-c)^{n},(x-d)^{m}(y-d)^{n}\right]
$$

for arbitrary constants $a, b, c$ and $d$. Then we see that

$$
\begin{array}{r}
z^{1}=z_{y}+\frac{n}{x-y} z=\frac{n}{x-y}\left[(x-a)^{m+1}(y-a)^{n-1},(x-b)^{m+1}(y-b)^{n-1},\right. \\
\left.(x-c)^{m+1}(y-c)^{n-1},(x-d)^{m+1}(y-d)^{n-1}\right] .
\end{array}
$$

Remark 4.9. Chern [Ch44, Ch47] first considered the Laplace transformation when the dimension $n$ is greater than 2. A systematic new treatment was given in [KT96].

\subsection{Recursive relations of Laplace invariants}

It is interesting to know how the Laplace invariants vary with Laplace transformations. We denote by $z^{i}$ the successive transforms, namely $z^{i \pm 1}=\left(z^{i}\right)^{ \pm 1}$, and we denote the Laplace invariants of $z^{i}$ by $h_{i}$ and $k_{i}$. The sequence $\left\{z^{i}\right\}$, when well-defined, is called the Laplace sequence.

PROPOSITION 4.10. The Laplace invariants satisfy the following relations.

$$
\begin{aligned}
& h_{i+1}=2 h_{i}-k_{i}-\frac{\partial^{2} \log h_{i}}{\partial x \partial y}, \\
& k_{i+1}=h_{i}, \\
& k_{i}=2 k_{i+1}-h_{i+1}-\frac{\partial^{2} \log k_{i+1}}{\partial x \partial y} ; \\
& h_{i+1}+h_{i-1}=2 h_{i}-\frac{\partial^{2} \log h_{i}}{\partial x \partial y}, \\
& k_{i+1}+k_{i-1}=2 k_{i}-\frac{\partial^{2} \log k_{i}}{\partial x \partial y} ;
\end{aligned}
$$




$$
\begin{aligned}
h_{i+1} & =h_{i}+h-k-\left(\log \left(h h_{1} \cdots h_{i}\right)\right)_{x y}, \\
k_{i+1} & =k_{i}+h-k-\left(\log \left(k_{1} \cdots k_{i}\right)\right)_{x y} .
\end{aligned}
$$

Proof. It is enough to check the identities when $i=0$ for (1). Set $H=\log h$. Then we have, by (4.1) and (4.3),

$$
\begin{aligned}
h_{1} & =\left(a-H_{y}\right)_{x}-\left(c-a_{x}+b_{y}-b H_{y}\right)+b\left(a-H_{y}\right) \\
& =2\left(a b+a_{x}-c\right)-\left(a b+b_{y}-c\right)-H_{x y}, \\
& =2 h-k-H_{x y}, \\
k_{1} & =b_{y}-\left(c-a_{x}+b_{y}-b H_{y}\right)+b\left(a-H_{y}\right) \\
& =a b+a_{x}-c, \\
& =h,
\end{aligned}
$$

which yield (1). The identities (2) and (3) follow from (1).

We write the equation of $z^{i}$ as

$$
\left(z^{i}\right)_{x y}+a_{i}\left(z^{i}\right)_{x}+b_{i}\left(z^{i}\right)_{y}+c_{i} z=0 .
$$

The coefficients $a_{i}, b_{i}$, and $c_{i}$ are determined recursively by the formula (4.3). Namely, we see that

$$
\begin{gathered}
a_{i}=a_{i-1}-\left(\log h_{i-1}\right)_{y}, \\
b_{i}=b, \\
c_{i}=c_{i-1}-\left(a_{i-1}\right)_{x}+b_{y}-b\left(\log h_{i-1}\right)_{y},
\end{gathered}
$$

in the case $i>0$. A similar formula holds for the case $i<0$.

\subsection{Periodic Laplace sequences}

We say that a Laplace sequence $\left\{z^{i}\right\}$ is periodic of period $n$ if the surface $z^{n}$ coincides with the starting surface $z$. In this case, we have $h_{n}=h$ and $k_{n}=k$. We first give two examples.

Example 4.11. Assume that $(E)=\left(E_{1}\right)$, i.e. $h_{1}=h$ and $k_{1}=k$. Then, we get $k=h$ and $(\log h)_{x y}=0$. Hence, $h=X(x) Y(y)$. By a change of coordinates, we can assume that $h=1$, as long as $h \neq 0$. This means that the equation is nothing but

$$
z_{x y}=z
$$


Example 4.12. Assume that $(E)=\left(E_{2}\right)$, i.e. the transformation is doubly periodic, and $h k \neq 0$. We have $h_{2}=h$ and $k_{2}=k$. From Proposition 4.10(1) above,

$$
2 k-2 h=\frac{\partial^{2} \log h}{\partial x \partial y}, \quad 2 h-2 k=\frac{\partial^{2} \log k}{\partial x \partial y} .
$$

This implies that $(\log h k)_{x y}=0$; hence, $h k=X(x) Y(y)$ and we can find coordinates such that $h k=1$. In this case, by setting $h=e^{\theta}$, we have

$$
\frac{\partial^{2} \theta}{\partial x \partial y}=4 \sinh \theta
$$

Conversely, for any solution $\theta$ of this equation, let us define the equation $(E)$, where the coefficients $a, b$, and $c$ are defined by solving the equations

$$
a_{x}=h=e^{\theta}, \quad b_{y}=k=e^{-\theta}, \quad c=a b ;
$$

then we get a surface and its Laplace sequence that is doubly periodic.

We next treat the general periodic case. By assumption,

$$
z^{n}=\mu z
$$

for a non-vanishing function $\mu$. Assume that $h_{i} \neq 0$ in the following. By Proposition 4.10(3), it holds that

$$
\left(\log h h_{1} \cdots h_{n-1}\right)_{x y}=0 .
$$

Hence, $h h_{1} \cdots h_{n-1}=X(x) Y(y)$ for certain functions $X$ and $Y$. By a coordinate change, we can assume that

$$
h h_{1} \cdots h_{n-1}=1
$$

see Proposition 4.2. From (4.3),

$$
\begin{gathered}
b_{n}=b, \\
a_{n}=a_{n-1}-\left(\log h_{n-1}\right)_{x y}=\cdots=a-\left(\log h h_{1} \cdots h_{n-1}\right)_{x y}=a .
\end{gathered}
$$

From $h_{n}=h$, we see that $\left(a_{n}\right)_{x}+a_{n} b_{n}-c_{n}=a_{x}+a b-c$; hence, we also get $c_{n}=c$. Therefore, $z^{n}$ and $z$ satisfy the same equation and $\mu$ is constant. We can see, moreover, that

$$
\begin{gathered}
z^{n+i}=\mu z^{i} \quad \text { for } i>0, \\
z^{n-i}=\frac{\mu}{h_{n-1} h_{n-2} \cdots h_{n-i}} z^{-i} \text { for } 0<i<n .
\end{gathered}
$$

We restrict our further consideration to only the case $h=k$. 
Proposition 4.13. Assume that the sequence is $n$-periodic and $h=k$. Choose coordinates so that $h h_{1} \cdots h_{n-1}=1$. Then

(1) $\quad h_{2 p-i}=h_{i-1} \quad(i=1,2, \ldots, p) \quad$ when $n=2 p$,

(2) $\quad h_{2 p+1-i}=h_{i-1} \quad(i=1,2, \ldots, p) \quad$ when $n=2 p+1$.

Proof. For the case where $h=k$, Proposition 4.10(3) implies that

$$
h_{i}=h_{i-1}-\left(\log h h_{1} \cdots h_{i-1}\right)_{x y} .
$$

When $i=2 p-1$, we have

$$
h_{2 p-1}=h_{2 p}+\left(\log h h_{1} \cdots h_{2 p-1}\right)_{x y}=h_{2 p}=h .
$$

Assume that (1) holds for $i=1,2, \ldots, j$. Then

$$
h_{j-1}=h_{j-2}-\left(\log h h_{1} \cdots h_{j-2}\right)_{x y}=h_{j-2}-\left(\log h_{2 p-1} h_{2 p-2} \cdots h_{2 p-j+1}\right)_{x y} .
$$

Combining this with the identity above for $i=2 p-j$, we get the result for (1). The case (2) can be similarly shown.

Example 4.14. Consider the case where $p=1$ and $n=3$. As $h h_{1} h_{2}=1$ and $h_{2}=h$, we have $h_{1}=1 / h^{2}$. Hence, $h$ satisfies the equation

$$
(\log h)_{x y}=h-1 / h^{2} .
$$

The associated differential equation is

$$
z_{x y}=h z
$$

and the Laplace sequence satisfies

$$
z^{3}=m z, \quad z^{2}=(m / h) z^{-1}, \quad z^{1}=m h z^{-2} .
$$

On the other hand, by definition,

$$
z^{1}=z_{y}, \quad z^{2}=z_{y y}-(\log h)_{y} z_{y}, \quad z^{-1}=z_{x}, \quad z^{-2}=z_{x x}-(\log h)_{x} z_{x} .
$$

Therefore, $z$ satisfies a system of differential equations

$$
z_{x x}=\frac{h_{x}}{h} z_{x}+\frac{1}{m h} z_{y}, \quad z_{x y}=h z, \quad z_{y y}=\frac{m}{h} z_{x}+\frac{h_{y}}{h} z_{y} .
$$

As the number of independent solutions of this system is three, the surface $z$ lies in a plane in the projective space. 
Example 4.15. We next consider the case where $p=2$ and $n=4$. Here $h h_{1} h_{2} h_{3}=1$, $h_{2}=h_{1}$ and $h_{3}=h$. We may assume that $h_{1}=1 / h$. Then $h$ must satisfy

$$
(\log h)_{x y}=h-\frac{1}{h}
$$

and $z$ is a solution of the system of differential equations

$$
z_{x y}=h z, \quad z^{4}=m z, \quad z^{3}=\frac{m}{h} z^{-1}, \quad z^{2}=m z^{-2}, \quad z^{1}=m h z^{-3} .
$$

It is straightforward to see that this system is the same as

$$
z_{x y}=h z, \quad m z_{x x}-z_{y y}=m \frac{h_{x}}{h} z_{x}-\frac{h_{y}}{h} z_{y} .
$$

Conversely, for any $h$ satisfying (4.6), four independent solutions of (4.7) define a surface with the required periodicity and with equal Laplace invariants.

The simplest example of $h$ is $h=1$. The system is $z_{x y}=z$ and $z_{x x}-z_{y y}=0$; the associated mapping is projectively equivalent to $\left[e^{x+y}, e^{-x-y}, \cos (x-y)\right.$, $\sin (x-y)]$ in $\mathbb{P}^{3}$ with the homogeneous coordinates $[X, Y, Z, U]$. The surface is nothing but the quadric $X Y=Z^{2}+U^{2}$. Differentiating relative to $y$ successively, we get $z^{1}=z_{y}, z^{2}=z_{y y}, z^{3}=z_{y y y}$ and $z^{4}=z$. The surface $z^{1}$ is the quadric $-X Y=Z^{2}+U^{2}$.

Remark 4.16. A Laplace sequence $\left\{z^{i}\right\}$ is said to be self-projective if there exists a projective transformation $T$ and an integer such that $T z^{i}=z^{m+i}$ for any $i$. This is a more general notion than periodicity.

\subsection{Terminating Laplace sequences}

Historically, a special interest was paid to Laplace sequences that terminate in a finite number of steps, say $h_{i}=0$ but $h_{i-1} \neq 0$. We exhibit some of the treatment of this case. As in Remark 4.4(3), equation $\left(E_{i}\right)$ is written as

$$
\frac{\partial}{\partial x}\left(\frac{\partial z^{i}}{\partial y}+a_{i} z^{i}\right)+b_{i}\left(\frac{\partial z^{i}}{\partial y}+a_{i} z^{i}\right)=0 .
$$

The general solution has the form

$$
z^{i}=e^{-\int a_{i} d y}\left\{X(x)+\int^{y} Y(t) e^{\int a_{i} d t-\int b_{i} d x} d t\right\}
$$

where $X$ and $Y$ are arbitrary functions, so long as the integral is valid. Then the solution of the original equation is given by tracing through the Laplace transformations in reverse order. 
We assume $h_{i}=0$, where $i>0$, for simplicity. Then, by (4.2), equation ( $\left.E_{i}\right)$ is equivalent to

$$
z_{x y}+b z_{y}=0 \text {. }
$$

We define a function $\alpha$ by $b=-(\log \alpha)_{x}$ so that

$$
z_{x y}-(\log \alpha)_{x} z_{y}=0 .
$$

Then $h_{i-1}=k_{i}=-(\log \alpha)_{x y}$ by Proposition 4.10(1). Now

$$
h_{i-2}=2 h_{i-1}-\frac{\partial^{2} \log h_{i-1}}{\partial x \partial y}=-\frac{\partial^{2}}{\partial x \partial y} \log \left(\alpha \alpha_{x y}-\alpha_{x} \alpha_{y}\right) .
$$

Following Darboux, we introduce the notation

$$
D_{x}\left(\alpha_{1}, \ldots, \alpha_{m+1}\right)=\operatorname{det}\left(\begin{array}{cccc}
\alpha_{1} & \partial \alpha_{1} / \partial x & \ldots & \partial^{m} \alpha_{1} / \partial x^{m} \\
\vdots & \vdots & \ldots & \vdots \\
\alpha_{m+1} & \partial \alpha_{m+1} / \partial x & \cdots & \partial^{m} \alpha_{m+1} / \partial x^{m}
\end{array}\right) .
$$

Analogously, we introduce $D_{y}$. Then we set

$$
H_{m}=D_{x}\left(\alpha, \frac{\partial \alpha}{\partial y}, \ldots, \frac{\partial^{m} \alpha}{\partial y^{m}}\right)=D_{y}\left(\alpha, \frac{\partial \alpha}{\partial x}, \ldots, \frac{\partial^{m} \alpha}{\partial x^{m}}\right) .
$$

LEMMA 4.17. For $m \geq 0$, we have

$$
h_{i-m}=-\frac{\partial^{2} \log H_{m-1}}{\partial x \partial y} .
$$

Proof. By a formula for the expansion of determinants, we have

$$
H_{m-1} H_{m+1}=H_{m} \frac{\partial^{2} H_{m}}{\partial x \partial y}-\frac{\partial H_{m}}{\partial x} \frac{\partial H_{m}}{\partial y} .
$$

The right-hand side is equal to $H_{m}^{2}\left(\log H_{m}\right)_{x y}$. Hence, by derivation of both sides, we have

$$
\left(\log H_{m-1}\right)_{x y}+\left(\log H_{m+1}\right)_{x y}=2\left(\log H_{m}\right)_{x y}+\left(\log \left(\log H_{m}\right)_{x y}\right)_{x y} .
$$

This identity is the same as the recurrence formula satisfied by $h_{i}$, up to a sign:

$$
h_{m-1}+h_{m+1}=2 h_{m}-\left(\log h_{m}\right)_{x y} .
$$

Taking into account the expressions of $h_{i-1}$ and $h_{i-2}$ given above, we have the conclusion. 
This lemma shows that the invariants $h$ and $k$ of equation $\left(E_{i-m}^{\prime}\right)$ are $-\left(\log H_{m-1}\right)_{x y}$ and $-\left(\log H_{m}\right)_{x y}$, respectively. The associated equation is

$$
z_{x y}-\left(\log H_{m-1}\right)_{y} z_{x}-\left(\log H_{m}\right)_{x} z_{y}+\left(\log H_{m-1}\right)_{y}\left(\log H_{m}\right)_{x} z=0 .
$$

Here we assume $H_{-1}=0$. For any solution $\theta$ of $\left(E_{i}^{\prime}\right)$, we define the function

$$
\theta_{m}=D_{x}\left(\theta, \alpha, \frac{\partial \alpha}{\partial y}, \ldots, \frac{\partial^{m-1} \alpha}{\partial y^{m-1}}\right), \quad m \geq 1 .
$$

Then we can assert the following.

Proposition 4.18. $\theta_{m}$ is a solution of $\left(E_{i-m}^{\prime}\right)$.

We refer to [D2, no 379; pp. 138-139] for the proof. If the Laplace sequence terminates on both sides, then we must have $\left(\log H_{m-1}\right)_{x y}=0$ for certain $m$. This implies that $H_{m}=D_{x}\left(\alpha, \partial \alpha / \partial y, \ldots, \partial^{m} \alpha / \partial y^{m}\right)=0$.

Hence, the $\alpha$ must satisfy an ordinary differential equation of order $m$ with coefficients being functions of $y$ alone. This property can be traced back to the starting equation $\left(E_{i}\right)$ and we can get a general description of solutions of $\left(E_{i}\right)$ in this case. We refer again to [D2, nos 382-386] for a detailed treatment.

\subsection{The Euler-Poisson-Darboux equation}

The Euler-Poisson-Darboux equation is by definition a differential equation of the form

$$
z_{x y}-\frac{n}{x-y} z_{x}-\frac{m}{y-x} z_{y}-\frac{p}{(x-y)^{2}} z=0,
$$

where $n, m$ and $p$ are constants. It is a special case of equation $(E)$.

When we set $z=(x-y)^{\alpha} w, w$ satisfies an equation of the same form with constants replaced by

$$
n \rightarrow n+\alpha, \quad m \rightarrow m+\alpha, \quad p \rightarrow p+\alpha^{2}+\alpha(m+n-1) .
$$

Hence, for an appropriate $\alpha$, we can reduce the equation to

$$
z_{x y}-\frac{\beta^{\prime}}{x-y} z_{x}-\frac{\beta}{y-x} z_{y}=0 .
$$

First we note that by setting $z=(x-y)^{1-\beta-\beta^{\prime}} w$ the equation for $w$ remains the same, but with coefficients replaced by

$$
\beta \rightarrow 1-\beta \quad \text { and } \quad \beta^{\prime} \rightarrow 1-\beta^{\prime}
$$

This is an involutive transformation from $E\left(\beta, \beta^{\prime}\right)$ to $E\left(1-\beta^{\prime}, 1-\beta\right)$. 
We next remark that the equation has special solutions, by use of Gauss hypergeometric functions. Let us set $y / x=t$ and assume $z$ has the form $z=x^{\lambda} \varphi(t)$. Then $\varphi$ satisfies the Gauss hypergeometric equation

$$
t(1-t) \varphi^{\prime \prime}+\left\{(1-\beta-\lambda)-\left(1+\beta^{\prime}-\lambda\right) t\right\} \varphi^{\prime}+\lambda \beta^{\prime} \varphi=0 .
$$

In particular, this implies that $E\left(\beta, \beta^{\prime}\right)$ has independent solutions

$$
x^{\lambda} F\left(-\lambda, \beta^{\prime}, 1-\beta-\lambda ; y / x\right) \text { and } \quad x^{-\beta} y^{\beta+\lambda} F\left(\beta, \beta+\beta^{\prime}+\lambda, 1+\beta+\lambda ; y / x\right),
$$

where $\lambda$ is an arbitrary constant and $F$ denotes the Gauss hypergeometric function. Furthermore, when $\lambda$ is a positive integer, the first solution is a homogeneous polynomial of degree $\lambda$.

The second remark we make is that the equation $E\left(\beta, \beta^{\prime}\right)$ has special solutions of the form $z=X(x) Y(y)$. Relative to this $z$, the equation is written as

$$
(x-y) X_{x} Y_{y}-\beta^{\prime} X_{x} Y+\beta X Y_{y}=0 .
$$

Hence, $x+\beta X / X_{x}=y+\beta^{\prime} Y / Y_{y}$ must be a constant $a$; we get

$$
X=(x-a)^{-\beta} \text { and } Y=(y-a)^{-\beta^{\prime}}
$$

up to multiplicative constants. Namely, $(x-a)^{-\beta}(y-a)^{-\beta^{\prime}}$ is a solution for any constant $a$. By the involution stated above,

$$
(y-x)^{1-\beta-\beta^{\prime}}(x-a)^{\beta^{\prime}-1}(y-a)^{\beta-1}
$$

is also a solution.

The third remark we make is on the symmetry of the equation. We easily see that the Laplace invariants are

$$
h=\frac{\beta^{\prime}(1-\beta)}{(x-y)^{2}} \quad \text { and } \quad k=\frac{\beta\left(1-\beta^{\prime}\right)}{(x-y)^{2}} .
$$

Let $(x, y) \rightarrow(s, t)$ be a coordinate transformation of the form

$$
x=\frac{a s+b}{c s+d} \quad \text { and } \quad y=\frac{a t+b}{c t+d} .
$$

As it holds that

$$
x-y=-\frac{a d-b c}{(c s+d)(c t+d)}(s-t), \quad d x=\frac{a d-b c}{(c s+d)^{2}} d s, \quad d y=\frac{a d-b c}{(c t+d)^{2}} d t,
$$


and because $h d x d y=h^{\prime} d s d t$ and $k d x d y=k^{\prime} d s d t$, where $h^{\prime}$ and $k^{\prime}$ are the invariants for the new equation relative to $(s, t)$, we see that

$$
h^{\prime}=\frac{\beta^{\prime}(1-\beta)}{(s-t)^{2}} \quad \text { and } \quad k^{\prime}=\frac{\beta\left(1-\beta^{\prime}\right)}{(s-t)^{2}} .
$$

This implies that, if we denote by $S\left(\beta, \beta^{\prime}\right)$ the space of solutions, then the space $S\left(\beta, \beta^{\prime}\right)$ has an $S L_{2}$-action. More precisely, we have the following.

Proposition 4.19. (Appell) For any solution $\varphi(x, y)$ of the equation $E\left(\beta, \beta^{\prime}\right)$, the function

$$
(a x+b)^{-\beta}(a y+b)^{-\beta^{\prime}} \varphi\left(\frac{c x+d}{a x+b}, \frac{c y+d}{a y+b}\right)
$$

is also a solution of $E\left(\beta, \beta^{\prime}\right)$, where $\left(\begin{array}{ll}a & b \\ c & d\end{array}\right)$ is any element of $S L_{2}$. The associated infinitesimal action is given by

$$
\begin{gathered}
X=\partial_{x}+\partial_{y}, \quad Y=x \partial_{x}+y \partial_{y}+\frac{1}{2}\left(\beta+\beta^{\prime}\right), \\
Z=x^{2} \partial_{x}+y^{2} \partial_{y}+\beta x+\beta^{\prime} y .
\end{gathered}
$$

The correspondence with the matrix elements is given by

$$
X \leftrightarrow\left(\begin{array}{ll}
0 & 1 \\
0 & 0
\end{array}\right), \quad Y \leftrightarrow\left(\begin{array}{cc}
1 & 0 \\
0 & -1
\end{array}\right) \quad \text { and } \quad Z \leftrightarrow\left(\begin{array}{ll}
0 & 0 \\
1 & 0
\end{array}\right)
$$

They satisfy the expected relations $[X, Z]=2 Y,[Y, X]=-X$ and $[Y, Z]=Z$.

So far, we have two kinds of symmetries of the family $\left\{E\left(\beta, \beta^{\prime}\right)\right\}$. We next give two other kinds of symmetries. The first is given by the Laplace transformation. Starting with the equation $E\left(\beta, \beta^{\prime}\right)$, let $E_{i}$ be the $i$ th Laplace transform with invariants $h_{i}$ and $k_{i}$. By use of Proposition 4.10, we easily see that

$$
h_{i}=\frac{\left(i+\beta^{\prime}\right)(i-\beta+1)}{(x-y)^{2}} \quad \text { and } \quad k_{i}=\frac{\left(i+\beta^{\prime}-1\right)(i-\beta)}{(x-y)^{2}}
$$

this means that $E_{i}=E\left(\beta-i, \beta^{\prime}+i\right)$.

The second symmetry is given by the operation that associates to each solution $z$ of $E\left(\beta, \beta^{\prime}\right)$ the derivative $\partial z / \partial x$. Differentiating the equation $E\left(\beta, \beta^{\prime}\right)$, it is direct to see that $\partial z / \partial x$ belongs to $S\left(\beta+1, \beta^{\prime}\right)$. Similarly, $\partial z / \partial y$ belongs to $S\left(\beta, \beta^{\prime}+1\right)$.

Proposition 4.20. Assume that $\beta \neq 0$. Then the operator $\partial / \partial x$ is surjective from $S\left(\beta, \beta^{\prime}\right)$ to $S\left(\beta+1, \beta^{\prime}\right)$. 
Proof. Given $z^{1} \in S\left(\beta+1, \beta^{\prime}\right)$, it is necessary to solve $\partial z / \partial x=z^{1}$. If $z$ exists in $S\left(\beta, \beta^{\prime}\right)$, then $(x-y) \partial z^{1} / \partial y-\beta^{\prime} z^{1}+\beta z_{y}=0$. Hence, we must have

$$
d z=z^{1} d x+\left(\frac{\beta^{\prime}}{\beta} z^{1}-\frac{x-y}{\beta} z_{y}^{1}\right) d y .
$$

The integrability of this Pfaff equation is nothing but the condition $z^{1} \in S\left(\beta+1, \beta^{\prime}\right)$, as can be seen by taking exterior differentiation.

Remark 4.21. The general solution of the exceptional equation $E\left(0, \beta^{\prime}\right)$ is seen to be of the form $\int^{x} X(t)(y-t)^{-\beta^{\prime}} d t+Y(y)$ in case $\beta^{\prime} \neq 0$, while $E(0,0)$ is the trivial equation $z_{x y}=0$. As for the equation $E\left(1, \beta^{\prime}\right)$, the first invariant vanishes. Considering the involution between $E\left(1, \beta^{\prime}\right)$ and $E\left(1-\beta^{\prime}, 0\right)$, we get a general solution of the form

$$
(x-y)^{-\beta^{\prime}}\left\{X(x)+\int^{y} Y(t)(x-t)^{\beta^{\prime}-1} d t\right\} .
$$

When both $\beta$ and $\beta^{\prime}$ are integers, the equation $E(1,1)$ is important. For any solution $z$ of this case, we set $w=(x-y) z$. Then it is straightforward to see that $w_{x y}=0$. Hence, the general solution of $E(1,1)$ is of the form $(X(x)-Y(y)) /(x-y)$.

Now we consider a more general case: the case where $0<\operatorname{Re} \beta<1$ and $0<\operatorname{Re} \beta^{\prime}<1$. The formula called Poisson-Appell treats this case and asserts that the general solution has the form

$$
\int^{y} \varphi(u)(u-x)^{-\beta}(y-u)^{-\beta^{\prime}} d s+(x-y)^{1-\beta-\beta^{\prime}} \int_{x}^{y} \psi(u)(u-x)^{\beta^{\prime}-1}(y-u)^{\beta-1} d s,
$$

in the case $\beta+\beta^{\prime} \neq 1$ and

$$
\begin{aligned}
\int_{0}^{1} \varphi(x+ & (y-x) t) t^{-\beta}(1-t)^{\beta-1} d t \\
& +\int_{0}^{1} \psi(x+(y-x) t) t^{-\beta}(1-t)^{\beta-1} \log (t(1-t)(y-x)) d t,
\end{aligned}
$$

in the case $\beta+\beta^{\prime}=1$, where $\varphi$ and $\psi$ are arbitrary functions, as long as the integral can be defined. The proof is given by using the connection formula of the Gauss hypergeometric functions. We refer to [D2, no. 362] for an elegant theory on the Euler-Poisson-Darboux equation.

Remark 4.22. We refer the reader to [D2] for Sections 4.1-4.6. 


\subsection{The Échell of hypergeometric functions}

We now make a short digression and discuss Appell's hypergeometric system, which gives an example of the Euler-Poisson-Darboux equation.

Appell's system denoted by $\left(F_{1}\right)$ is a system defined by

$$
\begin{aligned}
\theta\left(\theta+\theta^{\prime}+\gamma-1\right) z-x\left(\theta+\theta^{\prime}+\alpha\right)(\theta+\beta) z & =0, \\
\theta^{\prime}\left(\theta+\theta^{\prime}+\gamma-1\right) z-y\left(\theta+\theta^{\prime}+\alpha\right)\left(\theta+\beta^{\prime}\right) z & =0,
\end{aligned}
$$

where $\theta=x \partial_{x}, \theta^{\prime}=y \partial_{y}$ and $\alpha, \beta, \beta^{\prime}$ and $\gamma$ are complex parameters. Appell's hypergeometric function denoted by $F_{1}$ is a solution that is holomorphic around the origin:

$$
F_{1}\left(\alpha, \beta, \beta^{\prime}, \gamma ; x, y\right)=\sum_{m, n \geq 0} \frac{(\alpha, m+n)(\beta, m)\left(\beta^{\prime}, n\right)}{(\gamma, m+n)(1, m)(1, n)} x^{m} y^{n} ;
$$

we use here the notation $(a, m)=a(a+1) \cdots(a+m-1)$. By a straightforward computation, we see that any solution $z$ of $\left(F_{1}\right)$ satisfies the Euler-Poisson-Darboux equation $E\left(\beta, \beta^{\prime}\right)$. Hence, the first Laplace transform is

$$
w=z_{y}-\frac{\beta^{\prime}}{x-y} z
$$

and $w$ itself satisfies the system $\left(F_{1}\right)$ with different parameters. Relative to the function $F_{1}$, the transformation can be interpreted by the identity

$$
\left[\beta^{\prime}-(x-y) \partial_{y}\right] F_{1}\left(\alpha, \beta, \beta^{\prime}, \gamma ; x, y\right)=\beta^{\prime} F_{1}\left(\alpha, \beta-1, \beta^{\prime}+1, \gamma ; x, y\right),
$$

called the contiguity relation of Appell's system. In particular, we can prove that the solution space of $\left(F_{1}\right)$ is invariant under Laplace transformations.

Let us describe some details. For the pair $A=\left(\beta, \beta^{\prime}\right)$ we introduce the notation $A+1=\left(\beta-1, \beta^{\prime}+1\right)$, in view of the translation in (4.8) of parameters. We define

$$
\begin{gathered}
D(A)=(x-y) \partial_{x}+\beta, \quad U(A)=(x-y) \partial_{y}-\beta^{\prime}, \\
L(A)=\partial_{x} \partial_{y}-\frac{\beta^{\prime}}{x-y} \partial_{x}+\frac{\beta}{x-y} \partial_{y} .
\end{gathered}
$$

Then we see that

$$
\begin{aligned}
& D(A+1) U(A)=\beta^{\prime}(1-\beta)+(x-y)^{2} L(A), \\
& U(A-1) D(A)=\beta\left(1-\beta^{\prime}\right)+(x-y)^{2} L(A) .
\end{aligned}
$$

Hence, if we denote $F_{1}\left(\alpha, \beta, \beta^{\prime}, \gamma ; x, y\right)$ by $F(A)$, then

$$
D(A) F(A)=\beta F(A-1), \quad U(A) F(A)=-\beta^{\prime} F(A+1) .
$$

These identities show that the system is invariant under Laplace transformations. 
Using this invariance, Okamoto [088] gave a solution of the Toda equation as follows: To make the formula simpler, let us define

$$
\begin{gathered}
f_{n}=1 /(n+1-\beta), \quad g_{n}=1 /\left(n-1+\beta^{\prime}\right), \\
X=\partial_{x}, \quad Y=\partial_{y}, \\
L_{n}=X Y-\frac{\beta^{\prime}+n}{x-y} X+\frac{\beta-n}{x-y} Y,
\end{gathered}
$$

and set

$$
\begin{aligned}
& B_{n}=g_{n}((x-y) X+\beta-n)=g_{n} D(A+n), \\
& H_{n}=f_{n}\left((x-y) Y-\beta^{\prime}-n\right)=f_{n} U(A+n) .
\end{aligned}
$$

Then, introducing the series of functions $\left\{\phi_{n}\right\}$ by

$$
\phi_{0}=F(A), \quad \phi_{n}=H_{n} \phi_{0}
$$

we see that

$$
H_{n} \phi_{n}=\phi_{n+1}, \quad B_{n} \phi_{n}=\phi_{n-1} .
$$

In particular, defining

$$
\psi_{n}=(x-y)^{(\beta-n)\left(\beta^{\prime}+n\right)} \phi_{n}
$$

we finally have the following.

Proposition 4.23. We have

$$
f_{n} g_{n} X Y \log \psi_{n}=-\frac{\psi_{n+1} \psi_{n-1}}{\psi_{n}^{2}} .
$$

A similar formula of this kind also appears for the second Appell's system defined by

$$
D_{1} z=0 \quad \text { and } \quad D_{2} z=0
$$

where

$$
\begin{aligned}
& D_{1}=x(1-x) \partial_{x x}-x y \partial_{x y}+\{\gamma-(\alpha+\beta+1) x\} \partial_{x}-\beta y \partial_{y}-\alpha \beta, \\
& D_{2}=y(1-y) \partial_{y y}-x y \partial_{x y}+\left\{\gamma^{\prime}-\left(\alpha+\beta^{\prime}+1\right) y\right\} \partial_{y}-\beta^{\prime} x \partial_{x}-\alpha \beta^{\prime} .
\end{aligned}
$$

The following series is a solution that is holomorphic around the origin.

$$
F_{2}\left(\alpha, \beta, \beta^{\prime}, \gamma, \gamma^{\prime} ; x, y\right)=\sum_{m, n \geq 0} \frac{(\alpha, m+n)(\beta, m)\left(\beta^{\prime}, n\right)}{(\gamma, m)\left(\gamma^{\prime}, n\right)(1, m)(1, n)} x^{m} y^{n} .
$$


The system has four independent solutions and any set of independent solutions defines a surface in $\mathbb{P}^{3}$. The surface is uniquely defined up to a projective transformation and it has a unique conformal structure, a non-degenerate quadratic form $y /(1-x) d x^{2}+2 d x d y+x /(1-y) d y^{2}$. Relative to this form, one set of conjugate directions is given by $x \partial_{x}$ and $(x-1) \partial_{x}+y \partial_{y}$; see Appendix A. In view of this fact, we define two operators by

$$
\begin{gathered}
H_{n}=x \partial_{x}+(\beta+n), \\
B_{n}=\frac{1}{(\beta+n-1)(\beta-\gamma+n)}\left(x\left((x-1) \partial_{x}+y \partial_{y}\right)+\alpha x+(\beta+n)-\gamma\right) .
\end{gathered}
$$

Then

$$
B_{n+1} H_{n}-1=a_{n} L(\beta+n), \quad H_{n+1} B_{n}-1=b_{n} L(\beta+n),
$$

where

$$
a_{n}=\frac{\alpha x+\beta+n-\gamma}{(\beta+n-1)(\beta+n-\gamma)}, \quad b_{n}=\frac{x}{(\beta+n-1)(\beta+n-\gamma)} .
$$

The corresponding contiguity relation is

$$
H_{n} F_{2}\left(\alpha, \beta+n, \beta^{\prime}, \gamma, \gamma^{\prime} ; x, y\right)=(\beta+n) F_{2}\left(\alpha, \beta+n+1, \beta^{\prime}, \gamma, \gamma^{\prime} ; x, y\right) \text {. }
$$

If we set

$$
\chi_{n}=c_{n}(x-1)^{n} \Gamma(\beta+n) F_{2}\left(\alpha, \beta+n, \beta^{\prime}, \gamma, \gamma^{\prime} ; x, y\right),
$$

where $c_{n}$ is a certain constant depending on $n$, then we have

$$
\partial_{x}\left((x-1) \partial_{x}+y \partial_{y}\right) \log \chi_{n}=\frac{\chi_{n+1} \chi_{n-1}}{\chi_{n}^{2}} .
$$

\subsection{Godeaux sequences}

Godeaux gave a method for studying projective surfaces through their Plücker images in $\mathbb{P}^{5}$. His method relies on the consideration of the Laplace sequence associated with the Plücker image, called the Godeaux sequence. In this section, we show a charaterization of Demoulin surfaces by Godeaux sequence and a characterization of the Plücker image of surfaces in view of distinguished choices of frames of $\mathbb{P}^{5}$.

Given a surface by the system (2.12), we define two vectors in $\mathbb{P}^{5}$ by

$$
U=z \wedge z_{x}, \quad V=z \wedge z_{y} .
$$

It is easy to see that

$$
U_{x}=b V, \quad V_{y}=c U,
$$


and that $U$ and $V$ satisfy the Laplace equations:

$$
U_{x y}=(\log b)_{y} U_{x}+b c U, \quad V_{x y}=(\log c)_{x} V_{y}+b c V .
$$

By following the procedure given in Section 4.2, the first Laplace transform of $U$ relative to the coordinate $y$ is given by

$$
U^{1}=U_{y}-(\log b)_{y} U .
$$

It satisfies

$$
U_{x}^{1}=h_{1} U, \quad U_{x y}^{1}=h_{1} U^{1}+\left(\log b h_{1}\right)_{y} U_{x}^{1}, \quad h_{1}=2 \kappa_{1},
$$

where $\kappa_{1}=\left(b c-(\log b)_{x y}\right) / 2$ was defined in (2.17). Continuing this process successively, we can define

$$
U^{n+1}=U_{y}^{n}-\left(\log b h_{1} \cdots h_{n}\right)_{y} U^{n},
$$

where $h_{n}$ is defined by

$$
h_{n}=h_{n-1}-\left(\log b h_{1} \cdots h_{n-1}\right)_{x y}
$$

and $U^{n}$ satisfies

$$
U_{x y}^{n}=h_{n} U^{n}+\left(\log b h_{1} \cdots h_{n}\right)_{y} U_{x}^{n} .
$$

Similarly for $V$, we have a recursive definition

$$
\begin{gathered}
V^{n+1}=V_{x}^{n}-\left(\log c k_{1} \cdots k_{n}\right)_{x} V^{n}, \quad k_{1}=2 \kappa_{2}, \\
k_{n}=k_{n-1}-\left(\log c k_{1} \cdots k_{n-1}\right)_{x y} \\
V_{x y}^{n}=k_{n} V^{n}+\left(\log c k_{1} \cdots k_{n}\right)_{x} V_{y}^{n}
\end{gathered}
$$

where $\kappa_{2}=\left(b c-(\log c)_{x y}\right) / 2$. The sequence $\left\{\ldots, U^{n}, \ldots, U^{1}, U, V, V^{1}, \ldots\right.$, $\left.V^{n}, \ldots\right\}$ is called the Godeaux sequence. This sequence has a special property for a Demoulin surface, which we now describe.

By Lemma 2.12 for a Demoulin surface, we see that

$$
h_{4}=k_{1}, \quad h_{3}=b c, \quad h_{2}=h_{1} ; \quad k_{2}=k_{1}, \quad k_{3}=b c, \quad k_{4}=h_{1} .
$$

(Recall that we have set $h_{1}=2 \kappa_{1}$ and $k_{1}=2 \kappa_{2}$.) This tells us that some periodicity occurs in the sequence.

In order to look into the details, we introduce four vectors

$$
M_{1}=z \wedge z_{x y}, \quad M_{2}=z_{x} \wedge z_{y}, \quad M_{3}=z_{x} \wedge z_{x y}, \quad M_{4}=z_{y} \wedge z_{x y},
$$


following Godeaux [G34]. Then, by defining a frame $T={ }^{\mathrm{t}}\left(U, V, M_{1}, M_{2}, M_{3}, M_{4}\right)$ in $\mathbb{P}^{6}$, we see that

$$
\begin{aligned}
T_{x} & =\left(\begin{array}{cccccc}
0 & b & 0 & 0 & 0 & 0 \\
0 & 0 & 1 & 1 & 0 & 0 \\
b c & b_{y}+p & 0 & 0 & 1 & 0 \\
0 & p & 0 & 0 & 1 & 0 \\
-b q-p_{y} & 0 & p & b_{y}+p & 0 & b \\
0 & -b q-p_{y} & 0 & -b c & 0 & 0
\end{array}\right) T \\
T_{y} & =\left(\begin{array}{cccccc}
0 & 0 & 1 & -1 & 0 & 0 \\
c & 0 & 0 & 0 & 0 & 0 \\
c_{x}+q & b c & 0 & 0 & 0 & 1 \\
-q & 0 & 0 & 0 & 0 & -1 \\
-c p-q_{x} & 0 & 0 & b c & 0 & 0 \\
0 & -c p-q_{x} & q & -c_{x}-q & c & 0
\end{array}\right) T .
\end{aligned}
$$

From these relations, we can express $U^{1}, U^{2}, V^{1}$ and $V^{2}$ in terms of the six vectors above.

$$
\begin{gathered}
U^{1}=-(\log b)_{y} U+M_{1}-M_{2}, \\
U^{2}=\lambda U+b c V-\left((\log b)_{y}+K_{1 y}\right)\left(M_{1}-M_{2}\right)+2 M_{4}, \\
V^{1}=-(\log c)_{x} V+M_{1}+M_{2}, \\
V^{2}=b c U+\mu V-\left((\log c)_{x}+K_{2 x}\right)\left(M_{1}+M_{2}\right)+2 M_{3},
\end{gathered}
$$

where

$$
\lambda=2 Q+\frac{b_{y}^{2}}{2 b^{2}}+(\log b)_{y} K_{1 y}, \quad \mu=2 P+\frac{c_{x}^{2}}{2 c^{2}}+(\log c)_{x} K_{2 x},
$$

and where $P$ and $Q$ are given in (2.18) and $K_{1}$ and $K_{2}$ are given in (2.31). As $U$, $V, M_{1}, M_{2}, M_{3}$ and $M_{4}$ are independent, as we assumed that the surface is nondegenerate, there are no linear relations among $U, V, U^{1}, U^{2}, V^{1}$ and $V^{2}$, as can be seen from the above expressions. Now a slightly long computation gives the formulas

$$
\begin{aligned}
U^{3}= & -\left(\left(\log b h_{2}\right)_{y}+2 K_{1 y}\right) U^{2}+\left(4 Q-K_{1 y y}-(\log b)_{y} K_{1 y}-\left(K_{1 y}\right)^{2}\right) U^{1} \\
& +\left(4(\log b)_{y} Q+2 Q_{y}\right) U-4 c P V+c K_{2 x} V^{1}+c V^{2}
\end{aligned}
$$




$$
\begin{aligned}
V^{3}= & -\left(\left(\log c k_{2}\right)_{x}+2 K_{2 x}\right) V^{2}+\left(4 P-K_{2 x x}-(\log c)_{x} K_{2 x}-\left(K_{2 x}\right)^{2}\right) V^{1} \\
& +\left(4(\log c)_{x} P+2 P_{x}\right) V-4 b Q U+b K_{1 y} U^{1}+b U^{2} .
\end{aligned}
$$

THEOREM 4.24. Assume that $b c \neq 0$ and $\kappa_{1} \kappa_{2} \neq 0$. Then the Godeaux sequence is periodic with period six if and only if the surface is Demoulin.

Proof. If the surface is Demoulin, we saw that $P=Q=0$. Lemma 2.12 says that the expressions above reduce to $U^{3}=c V^{2}$ and $V^{3}=b U^{2}$, which implies the periodicity. The converse statement also follows from the expressions above.

In general, any consecutive sequence of six vectors forms a frame in $\mathbb{P}^{5}$ as we have seen. We modify the frame $T$ by introducing the vectors

$$
\begin{aligned}
& N_{1}=z \wedge z_{x y}-z_{x} \wedge z_{y}=M_{1}-M_{2}=U_{y}, \\
& N_{2}=z \wedge z_{x y}+z_{x} \wedge z_{y}=M_{1}+M_{2}=V_{x}, \\
& N_{3}=2 z_{y} \wedge z_{x y}+b c z \wedge z_{y}=2 M_{4}+b c V, \\
& N_{4}=2 z_{x} \wedge z_{x y}+b c z \wedge z_{x}=2 M_{3}+b c U .
\end{aligned}
$$

The set of vectors $\left\{U, V, N_{1}, \ldots, N_{4}\right\}$ are chosen to be orthonormal in the following sense. Given two vectors $u$ and $v$ in $\stackrel{2}{\wedge} \mathbb{R}^{4}$, the product $u \wedge v$ is a vector lying in $\stackrel{4}{\wedge} \mathbb{R}^{4}$ which we identity with the scalar field $\mathbb{R}$ and we get a scalar value $(u, v)=$ $u \wedge v$. We thus have an inner product on the space $\stackrel{2}{\wedge} \mathbb{R}^{4}$. By the identification $z \wedge z_{x} \wedge z_{y} \wedge z_{x y}=-\frac{1}{2}$, we can see that

$$
\left(U, N_{3}\right)=-1, \quad\left(V, N_{4}\right)=1, \quad\left(N_{1}, N_{1}\right)=1, \quad\left(N_{2}, N_{2}\right)=-1
$$

and the remaining products vanish. In particular, they are linearly independent and we thus get a new frame $\mathcal{T}={ }^{\mathrm{t}}\left(U, V, N_{1}, N_{2}, N_{3}, N_{4}\right)$ in the space $\mathbb{P}^{5}$. We remark that the signature of the inner product is $(3,3)$.

The frame $\mathcal{T}$ satisfies a Pfaff system

$$
d \mathcal{T}=\omega \mathcal{T}
$$

where

$$
\omega=\left(\begin{array}{cccccc}
0 & b d x & d y & 0 & 0 & 0 \\
c d y & 0 & 0 & d x & 0 & 0 \\
b c d x+\kappa d y & b_{y} d x & 0 & 0 & d y & 0 \\
c_{x} d y & b c d y+\delta d x & 0 & 0 & 0 & d x \\
0 & \mu d x+v d y & b c d x+\kappa d y & -c_{x} d y & 0 & c d y \\
\mu d x+v d y & 0 & -b_{y} d x & b c d y+\delta d x & b d x & 0
\end{array}\right)
$$


and

$$
\kappa=2 q+c_{x}, \quad \delta=2 p+b_{y}, \quad \mu=b_{y y}-b \kappa, \quad v=c_{x x}-c \delta .
$$

The integrability condition $d \omega=\omega \wedge \omega$ is

$$
\kappa_{x}=(b c)_{y}+c b_{y}, \quad \delta_{y}=(b c)_{x}+b c_{x}, \quad v_{x}-\mu_{y}=\delta c_{x}-\kappa b_{y},
$$

which is the same as the integrability condition of (2.13).

Let us make a simple consideration on the frame $\mathcal{T}$. By definition we have

$$
U_{x}=b V \text { and } V_{y}=c U, \quad N_{1}=U_{y} \text { and } N_{2}=V_{x} .
$$

While the line $\overline{U V}$ is lying in the quadric $Q_{4}=\left\{u \in \mathbb{P}^{5} \mid(u, u)=0\right\}$, the points $N_{1}$ and $N_{2}$ are lying outside the quadric. We next have

$$
d N_{1} \equiv d y N_{3}, \quad d N_{2} \equiv d x N_{4} \quad(\bmod U, V) .
$$

Hence, both $\left(N_{1}\right)_{x}$ and $\left(N_{2}\right)_{y}$ lie on the line $\overline{U V}$ and

$$
\left(N_{1}\right)_{y} \equiv N_{3}, \quad\left(N_{2}\right)_{x} \equiv N_{4} \quad(\bmod U, V)
$$

We further see that

$$
\left(N_{3}\right)_{x}=\mu V+b c N_{1} \equiv b c N_{1}, \quad\left(N_{4}\right)_{y}=\nu U+b c N_{2} \equiv b c N_{2} \quad(\bmod U, V) .
$$

We conversely start with a projective frame $t={ }^{\mathrm{t}}\left(t_{1}, \ldots, t_{6}\right)$ of $\mathbb{P}^{5}$ depending on two parameters $(x, y)$ such that it satisfies the orthonormality such as $\left(t_{i}, t_{j}\right)=h_{i j}$, where $h=\left(h_{i j}\right)$ is a non-degenerate constant matrix of signature $(3,3)$. Let us denote by $s o(h)$ the Lie algebra of the orthogonal group relative to $h$, which is isomorphic to $\operatorname{so}(3,3 ; \mathbb{R})$. Then the equation of motion satisfied by $t$ is written as

$$
d t=\omega t, \quad d t_{i}=\sum_{j} \omega_{i}^{j} t_{j}
$$

where $\omega$ is a 1 -form with values in $s o(h)$. For a given $t$ to be a frame associated to a projective surface, we impose the conditions that

$$
d t_{1} \equiv 0 \quad\left(\bmod t_{1}, t_{2}, t_{3}\right), \quad d t_{2} \equiv 0 \quad\left(\bmod t_{1}, t_{2}, t_{4}\right)
$$

and that

$$
\omega_{1}^{3}=: \omega^{2} \text { and } \omega_{2}^{4}=: \omega^{1} \text { are linearly independent. }
$$


In order that the line $\overline{t_{1} t_{2}}$ lies in the quadric $Q_{4}$ and the frame satisfies the property (4.11), we further impose the condition that

$$
h=\left(\begin{array}{ccc} 
& & -J \\
-J & &
\end{array}\right), \quad J=\left(\begin{array}{rr}
1 & 0 \\
0 & -1
\end{array}\right) .
$$

With these conditions, the frame $t$ can be normalized. We say a change of frame $t \rightarrow g t$ is allowable if $g h^{\mathrm{t}} g=h$. Then we have the following theorem.

THEOREM 4.25. There is an allowable change of frame so that the form $\omega$ has the form given in (4.12).

Proof is given by a detailed examination of the integrability, which will be given in Appendix C, where we also give a remark on the analogous presentation relative to Lie sphere geometry of Euclidean surfaces.

Remark 4.26. For the present section, see [G34] and [Fe00a].

\section{Affine spheres and the Laplace transformation}

In previous sections we defined several notions necessary in the following study of surfaces. In this section, apart from the projective treatment of surfaces, we examine the Laplace transformation of surfaces in the affine setting. To do this, we first recall the historical notion of affine spheres, called $S$-surfaces by Tzitzeica, and then explain a transformation of affine spheres called the Tzitzeica transformation. We fix one affine chart of projective space $\mathbb{P}^{3}$, which we denote by $\mathbf{A}^{3}$.

\subsection{Affine surfaces}

We consider an immersed surface given by a mapping $(x, y) \mapsto z(x, y) \in \mathbf{A}^{3}$. For a mapping to define a surface, the three vectors $z, z_{x}$, and $z_{y}$ must be independent, and then we have a system of the form

$$
\begin{gathered}
z_{x x}=a z_{x}+b z_{y}+c z, \\
z_{x y}=a^{\prime} z_{x}+b^{\prime} z_{y}+c^{\prime} z, \\
z_{y y}=a^{\prime \prime} z_{x}+b^{\prime \prime} z_{y}+c^{\prime \prime} z .
\end{gathered}
$$

Each coordinate of $z$ satisfies this system. Conversely, given a system as above, any set of three independent solutions defines an immersion, thus defining a surface. 
Two sets of independent solutions differ only by an affine transformation leaving the origin fixed; hence, the system defines a surface uniquely up to an affine motion preserving the origin. The conformal structure of the surface is well-defined and is given by

$$
c d x^{2}+2 c^{\prime} d x d y+c^{\prime \prime} d y^{2} .
$$

(See Section 5.4.) We assume that this symmetric 2-form is non-degenerate. When the coefficient field is the real number field, we assume further that the form is indefinite, because we need a separate treatment for the definite case. Then the coordinates $(x, y)$ are asymptotic relative to this form if $c=c^{\prime \prime}=0$, which in the following we fix once and for all.

Proposition 5.1. The equation $z_{x y}=a^{\prime} z_{x}+b^{\prime} z_{y}+c^{\prime} z$ has equal Laplace invariants.

Proof. We compute the integrability conditions of the system (5.1) by using the identities

$$
\left(z_{x x}\right)_{y}=\left(z_{x y}\right)_{x} \text { and }\left(z_{x y}\right)_{y}=\left(z_{y y}\right)_{x} .
$$

The former implies that

$$
\begin{gathered}
a_{y}+a^{\prime \prime} b=a_{x}^{\prime}+a^{\prime} b^{\prime}+c^{\prime}, \\
b_{y}+a b^{\prime}+b b^{\prime \prime}=b_{x}^{\prime}+a^{\prime} b+b^{\prime 2}, \\
a c^{\prime}=c_{x}^{\prime}+b^{\prime} c^{\prime},
\end{gathered}
$$

and the latter implies that

$$
\begin{gathered}
b_{x}^{\prime \prime}+a^{\prime \prime} b=b_{y}^{\prime}+a^{\prime} b^{\prime}+c^{\prime}, \\
a_{y}^{\prime}+a^{\prime 2}+a^{\prime \prime} b^{\prime}=a_{x}^{\prime \prime}+a a^{\prime \prime}+b^{\prime \prime} a^{\prime}, \\
b^{\prime \prime} c^{\prime}=c_{y}^{\prime}+a^{\prime} c^{\prime} .
\end{gathered}
$$

Hence, we get $b^{\prime}=a-\left(\log c^{\prime}\right)_{x}$ and $a^{\prime}=b^{\prime \prime}-\left(\log c^{\prime}\right)_{y}$. In particular, we have $b_{y}^{\prime}=a_{y}-\left(\log c^{\prime}\right)_{x y}$ and $a_{x}^{\prime}=b_{x}^{\prime \prime}-\left(\log c^{\prime}\right)_{x y}$. Hence, $a_{x}^{\prime}-b_{y}^{\prime}=b_{x}^{\prime \prime}-a_{y}$. On the other hand, the first equations of each of the two sets of equations above imply that $a_{x}^{\prime}-b_{y}^{\prime}=a_{y}-b_{x}^{\prime \prime}$. Therefore, $a_{x}^{\prime}=b_{y}^{\prime}$, which shows that the Laplace invariants $h$ and $k$ are equal.

By this proposition, we can set

$$
a^{\prime}=\lambda_{y} / \lambda \text { and } b^{\prime}=\lambda_{x} / \lambda
$$

for some function $\lambda$. 


\subsection{Affine spheres}

We next fix a Euclidean structure on the affine space $\mathbf{A}^{3}$. Let $E d x^{2}+2 F d x d y+$ $G d y^{2}$ be the usual induced metric and set $\Delta=\left(E G-F^{2}\right)^{1 / 2}$. The induced second fundamental form is

$$
\frac{1}{\Delta}\left\{\left|z_{x x} z_{x} z_{y}\right| d x^{2}+2\left|z_{x y} z_{x} z_{y}\right| d x d y+\left|z_{y y} z_{x} z_{y}\right| d y^{2}\right\},
$$

which is equal to $\left(2 c^{\prime} / \Delta\right)\left|z \quad z_{x} z_{y}\right| d x d y$ in the notation of the previous section. Let $d=d(x, y)$ be the distance from the origin to the tangent plane at $z(x, y)$. It is given by

$$
d=\frac{\left|z z_{x} z_{y}\right|}{\Delta} .
$$

Then, denoting the Gauss curvature by $K$, we get the formula

$$
\frac{K}{d^{4}}=-\frac{c^{\prime 2}}{\left|z z_{x} z_{y}\right|^{2}} \text {. }
$$

We define a scalar function $\mu$ by $\mu^{4}=\left|d^{4} / K\right|$. Then $\mu^{2} c^{\prime}= \pm\left|z z_{x} z_{y}\right|$, whose logarithmic derivative gives

$$
2 \frac{\mu_{x}}{\mu}+\frac{c_{x}^{\prime}}{c^{\prime}}=a+b^{\prime} \quad \text { and } \quad 2 \frac{\mu_{y}}{\mu}+\frac{c_{y}^{\prime}}{c^{\prime}}=b^{\prime \prime}+a^{\prime} .
$$

The identities $a=b^{\prime}+\left(\log c^{\prime}\right)_{x}$ and $b^{\prime \prime}=a^{\prime}+\left(\log c^{\prime}\right)_{y}$, which follow from (5.3) and (5.4), then imply that

$$
a^{\prime}=\mu_{y} / \mu \quad \text { and } \quad b^{\prime}=\mu_{x} / \mu,
$$

which together with (5.5) imply that $\mu=m \lambda$ for some constant $m$. We have seen that

$$
\frac{K}{d^{4}}=-\frac{1}{m^{4} \lambda^{4}} \text {. }
$$

COROLlARY 5.2. The quantity $K / d^{4}$ is uniquely determined from the system, up to a constant factor.

Definition 5.3. The surface $z$ is called an affine sphere if $K / d^{4}$ is constant.

This notion coincides with the definition of an affine sphere in affine differential geometry. We refer the reader to [NS94].

The discussion above shows that an affine sphere is defined by a system of the form

$$
z_{x x}=a z_{x}+b z_{y}, \quad z_{x y}=h z, \quad z_{y y}=a^{\prime \prime} z_{x}+b^{\prime \prime} z_{y}
$$


where $h=c^{\prime}$ because $\mu$ is constant. We know $h \neq 0$ by non-degeneracy. The integrability condition is the following:

$$
\begin{aligned}
& h_{x}=a h, \quad a_{y}+a^{\prime \prime} b=h, \quad b_{y}+b b^{\prime \prime}=0, \\
& h_{y}=b^{\prime \prime} h, \quad a_{x}^{\prime \prime}+a a^{\prime \prime}=0, \quad b_{x}^{\prime \prime}+a^{\prime \prime} b=h .
\end{aligned}
$$

Suppose that $b=0$. Then the surface is ruled with $x$ as the line coordinate. When $a^{\prime \prime}=0, y$ is the line coordinate. To exclude these cases, we assume $a^{\prime \prime} b \neq 0$. Then the integrability condition is written as

$$
a=\frac{h_{x}}{h}, \quad b^{\prime \prime}=\frac{h_{y}}{h}, \quad a^{\prime \prime}=\frac{Y(y)}{h}, \quad b=\frac{X(x)}{h}
$$

and

$$
(\log h)_{x y}=h-\frac{X(x) Y(y)}{h^{2}},
$$

where $X$ is a function of $x$ and $Y$ is a function of $y$. By suitably choosing coordinates, we can further assume $X$ and $Y$ are constants so that $X Y=1$. Namely, we have seen the following.

PROPOSITION 5.4. Any affine sphere is given, up to an affine motion, by a system of the form

$$
z_{x x}=\frac{h_{x}}{h} z_{x}+\frac{m}{h} z_{y}, \quad z_{x y}=h z, \quad z_{y y}=\frac{1}{m h} z_{x}+\frac{h_{y}}{h} z_{y},
$$

where $h$ is a solution of

$$
(\log h)_{x y}=h-\frac{1}{h^{2}}
$$

and $m$ is a constant.

Remark 5.5. We have already met the system above in Example 4.14 (see (4.4) and (4.5)).

Example 5.6. When $h=1$, the system is $z_{x x}=z_{y}, z_{x y}=z$, and $z_{y y}=z_{x}$. For any cubic root $\omega, e^{\omega x+\omega^{2} y}$ is a solution. The associated surface is $X Y Z=1$ in the complex affine space with coordinates $(X, Y, Z)$. In the real case, we also have a representation of the form $X\left(Y^{2}+Z^{2}\right)=1$.

\subsection{Affine polar surfaces}

Let $\mathbf{A}_{3}$ denote the dual space of $\mathbf{A}^{3}$ and $\langle\cdot, \cdot\rangle$ the dual pairing. Given a surface $z$ in $\mathbf{A}^{3}$, we define its polar surface $\zeta:(x, y) \mapsto \zeta(x, y) \in \mathbf{A}_{3}$ by requiring that

$$
\langle\zeta, z\rangle=1, \quad\left\langle\zeta, z_{x}\right\rangle=0, \quad\left\langle\zeta, z_{y}\right\rangle=0 .
$$


LEMMA 5.7. Assume that the surface $z$ is given by the system (5.1) with $c=c^{\prime \prime}=0$. Then the surface $\zeta$ is determined by the system

$$
\begin{gathered}
\zeta_{x x}=\left(a-2 b^{\prime}\right) \zeta_{x}-b \zeta_{y}, \\
\zeta_{x y}=-a^{\prime} \zeta_{x}-b^{\prime} \zeta_{y}+c^{\prime} \zeta^{\prime \prime} \\
\zeta_{y y}=-a^{\prime \prime} \zeta_{x}+\left(b^{\prime \prime}-2 a^{\prime}\right) \zeta_{y}
\end{gathered}
$$

Proof. Set

$$
\zeta_{x x}=p \zeta_{x}+q \zeta_{y}+r \zeta, \quad \zeta_{x y}=p^{\prime} \zeta_{x}+q^{\prime} \zeta_{y}+r^{\prime} \zeta, \quad \zeta_{y y}=p^{\prime \prime} \zeta_{x}+q^{\prime \prime} \zeta_{y}+r^{\prime \prime} \zeta
$$

By differentiation of the parings above, we can get the following. From $\langle\zeta, z\rangle=1$,

$$
\text { (1) }\left\langle\zeta_{x}, z\right\rangle=0,\left\langle\zeta_{y}, z\right\rangle=0
$$

From $\left\langle\zeta, z_{x}\right\rangle=0$ and $\left\langle\zeta, z_{y}\right\rangle=0$
(2) $\left\langle\zeta_{x}, z_{x}\right\rangle=0$,
(3) $\left\langle\zeta_{y}, z_{x}\right\rangle=\left\langle\zeta_{x}, z_{y}\right\rangle=-c^{\prime}$
(4) $\left\langle\zeta_{y}, z_{y}\right\rangle=0$.

Differentiating (1) again, we see that $\left\langle\zeta_{x x}, z\right\rangle=0,\left\langle\zeta_{x y}, z\right\rangle=c^{\prime},\left\langle\zeta_{y y}, z\right\rangle=0$; namely, $r=0, r^{\prime}=c^{\prime}$, and $r^{\prime \prime}=0$. Differentiating (2), we get $\left\langle\zeta_{x x}, z_{x}\right\rangle+\left\langle\zeta_{x}, z_{x x}\right\rangle$ $=0$ and $\left\langle\zeta_{x y}, z_{x}\right\rangle+\left\langle\zeta_{z}, z_{x y}\right\rangle=0$; both imply that $q=-b$ and $q^{\prime}=-b^{\prime}$. Similarly, from (4), we get $p^{\prime}=-a^{\prime}$ and $p^{\prime \prime}=-a^{\prime \prime}$. Differentiating (3), we get $\left\langle\zeta_{x x}, z_{y}\right\rangle+\left\langle\zeta_{x}, z_{x y}\right\rangle=c_{x}^{\prime}$, which implies that $p=-b^{\prime}+\left(\log c^{\prime}\right)_{x}$. Since $\left(\log c^{\prime}\right)_{x}=$ $a-b^{\prime}$ by the integrability, we see that $p=a-2 b^{\prime}$. Similarly, we have $q^{\prime \prime}=b^{\prime \prime}-2 a^{\prime}$.

COROLLARY 5.8. The asymptotic coordinates for the surface $z$ are also asymptotic for the polar surface $\zeta$. When $z$ is an affine sphere, with parameter $m$, the polar surface $\zeta$ is also an affine sphere, with parameter $-m$.

\subsection{From an affine surface to a projective surface}

Let $z^{1}, z^{2}$ and $z^{3}$ be independent solutions of the system (5.1). The surface $z(x, y)=$ $\left(z^{1}(x, y), z^{2}(x, y), x^{3}(x, y)\right)$ defines a surface in projective space by the mapping

$$
(x, y) \longrightarrow w(x, y)=\left[\lambda z^{1}, \lambda z^{2}, \lambda z^{3}, \lambda\right]=:\left[w^{1}, w^{2}, w^{3}, w^{4}\right],
$$


for an arbitrary non-vanishing scalar function $\lambda$. The system that has solutions $w^{i}$ is determined by the following

$$
\left|\begin{array}{lllll}
w & w_{x} & w_{y} & w_{x y} & w_{x x} \\
w^{1} & w_{x}^{1} & w_{y}^{1} & w_{x y}^{1} & w_{x x}^{1} \\
w^{2} & w_{x}^{2} & w_{y}^{2} & w_{x y}^{2} & w_{x x}^{2} \\
w^{3} & w_{x}^{3} & w_{y}^{3} & w_{x y}^{3} & w_{x x}^{3} \\
w^{4} & w_{x}^{4} & w_{y}^{4} & w_{x y}^{4} & w_{x x}^{4}
\end{array}\right|=0, \quad\left|\begin{array}{lllll}
w & w_{x} & w_{y} & w_{x y} & w_{y y} \\
w^{1} & w_{x}^{1} & w_{y}^{1} & w_{x y}^{1} & w_{y y}^{1} \\
w^{2} & w_{x}^{2} & w_{y}^{2} & w_{x y}^{2} & w_{y y}^{2} \\
w^{3} & w_{x}^{3} & w_{y}^{3} & w_{x y}^{3} & w_{y y}^{3} \\
w^{4} & w_{x}^{4} & w_{y}^{4} & w_{x y}^{4} & w_{y y}^{4}
\end{array}\right|=0,
$$

where $w$ is an indeterminate. As the projective class of the surface is independent of the choice of $\lambda$, we here assume for simplicity that $\lambda=1$. Then, the system is written as follows:

$$
\begin{gathered}
c^{\prime} w_{x x}=c w_{x y}+\left(c^{\prime} a-c a^{\prime}\right) w_{x}+\left(c^{\prime} b-c b^{\prime}\right) w_{y}, \\
c^{\prime} w_{y y}=c^{\prime \prime} w_{x y}+\left(c^{\prime} b^{\prime \prime}-c^{\prime \prime} b^{\prime}\right) w_{y}+\left(c^{\prime} a^{\prime \prime}-c^{\prime \prime} a^{\prime}\right) w_{x} .
\end{gathered}
$$

Hence, the conformal structure of the surface is $c d x^{2}+2 c^{\prime} d x d y+c^{\prime \prime} d y^{2}$, as we have already claimed.

If $c=c^{\prime \prime}=0$, then the system reduces to

$$
w_{x x}=a w_{x}+b w_{y}, \quad w_{y y}=a^{\prime \prime} w_{x}+b^{\prime \prime} w_{y} .
$$

In particular, for the system of affine spheres (5.6), the associated projective system is

$$
z_{x x}=\frac{h_{x}}{h} z_{x}+\frac{m}{h} z_{y}, \quad z_{y y}=\frac{1}{m h} z_{x}+\frac{h_{y}}{h} z_{y},
$$

with the additional condition (5.7): $(\log h)_{x y}=h-1 / h^{2}$.

\subsection{Laplace transforms of affine spheres}

Given an affine sphere $z$ by the system (5.6), we define its first Laplace transform

$$
w^{1}=\lambda z_{y}
$$

in the affine space, where $\lambda$ is a non-vanishing scalar function determined later. Assume here $m=1$ for simplicity. By computation,

$$
w_{x}^{1}=(\log \lambda)_{x} w^{1}+\lambda h z, \quad w_{y}^{1}=(\log \lambda h)_{y} w^{1}+\frac{\lambda}{h} z .
$$

Hence, $z$ and $z_{x}$ can be recovered by

$$
z=\frac{1}{\lambda h}\left(w_{x}^{1}-(\log \lambda)_{x} w^{1}\right), \quad z_{x}=\frac{h}{\lambda}\left(w_{y}^{1}-(\log \lambda h)_{y} w^{1}\right) .
$$


By differentiation, we get

$$
\begin{gathered}
w_{x x}^{1}=\left(\log \lambda^{2} h\right)_{x} w_{x}^{1}+h^{2} w_{y}^{1}+\left((\log \lambda)_{x x}-(\log \lambda h)_{x}(\log \lambda)_{x}-h^{2}(\log \lambda h)_{y}\right) w^{1}, \\
w_{x y}^{1}=(\log \lambda)_{x} w_{y}^{1}+(\log \lambda h)_{y} w_{x}^{1}+\left((\log \lambda)_{x y}-(\log \lambda h)_{y}(\log \lambda)_{x}+h\right) w^{1}, \\
w_{y y}^{1}=\frac{1}{h} w_{x}^{1}+\left(\log \lambda^{2}\right)_{y} w_{y}^{1}+\left((\log \lambda h)_{y y}-(\log (\lambda / h))_{y}(\log \lambda h)_{y}-\frac{1}{h}(\log \lambda)_{x}\right) w^{1} .
\end{gathered}
$$

From the second equation, the Laplace invariants of the surface $w$, which we denote by $h_{1}$ and $k_{1}$, are

$$
h_{1}=1 / h^{2}, \quad k_{1}=h .
$$

By definition, the second Laplace transform $w^{2}$ is given by

$$
w^{2}=\mu\left(w_{y}^{1}-(\log \lambda h)_{y} w^{1}\right)
$$

for a certain scalar $\mu$, and we see that this is equal to $(\lambda \mu / h) z_{x}$. By computation, we get

$$
\begin{gathered}
w_{x}^{2}=(\log \lambda \mu)_{x} w^{2}+\left(\mu / h^{2}\right) w^{1}, \\
w_{y}^{2}=\left((\log u)_{y}+(\log \lambda / h)_{y}+h(\log \lambda) x(\log \lambda \mu) x\right) w^{2}+(\mu / h) w_{x}^{1} \\
+(1 / h)(\log \lambda)_{x} w_{x}^{2} .
\end{gathered}
$$

Hence, we can write $w^{1}, w_{x}^{1}$, and $w_{y}^{1}$ in terms of $w^{2}$ as

$$
\begin{gathered}
w^{1}=\frac{h^{2}}{\mu}\left(w_{x}^{2}-(\log \lambda \mu)_{x} w^{2}\right), \\
w_{y}^{1}=\frac{h^{2}}{\mu}(\log \lambda h)_{y} w_{x}^{2}+\frac{1}{\mu}\left(1-h^{2}(\log \lambda h)_{y}(\log \lambda \mu)_{x}\right) w^{2}, \\
w_{x}^{1}=\frac{h}{\mu}\left(w_{y}^{2}-\frac{1}{h}(\log \lambda)_{x} w_{x}^{2}-\left((\log \lambda \mu / h)_{y}+h(\log \lambda)_{x}(\log \lambda \mu)_{x}\right) w^{2}\right) .
\end{gathered}
$$

From these relations we have

$$
\begin{aligned}
w_{x y}^{2}= & (\log \lambda \mu / h)_{y} w_{x}^{2}+(\log \lambda \mu)_{x} w_{y}^{2}+\left((\log \lambda \mu)_{x y}+1 / h^{2}\right. \\
& \left.-(\log \lambda \mu)_{x}(\log \lambda \mu / h)_{y}\right) w^{2} .
\end{aligned}
$$

This implies that the third Laplace transform is given by

$$
w^{3}=v\left(w_{y}^{2}-(\log \lambda \mu / h)_{y} w^{2}\right)
$$

for a certain scalar $v$, and the Laplace invariants $h_{2}$ and $k_{2}$ are

$$
h_{2}=h, \quad k_{2}=\frac{1}{h^{2}}
$$


Now it is easy to see that

$$
w^{3}=\lambda \mu \nu z
$$

PROPOSITION 5.9. Assume that $\lambda \mu \nu=1$. Then the affine sphere $z$ is 3-periodic relative to Laplace transformation.

\subsection{Tzitzeica transforms of affine spheres}

Tzitzeica found a transformation formula that sends a given affine sphere to a new affine sphere. We reproduce the formula in our setting.

We start by recalling the Moutard transformation of the equation

$$
z_{x y}=h z,
$$

which is a part of the system (5.6). For notational simplicity, we introduce the operator

$$
M(z)=\frac{z_{x y}}{z} .
$$

Then, equation (5.9) is simply written as $M(z)=h$. Let $R$ be an arbitrary scalar solution of (5.9) and define a 1-form $\omega$ associated to any solution $z$ by

$$
\omega=\left(R z_{x}-R_{x} z\right) d x-\left(R z_{y}-R_{y} z\right) d y .
$$

Then computation shows that $\omega$ is closed. This means that there is a function $u$ such that $\omega=d u$, namely,

$$
u_{x}=R z_{x}-R_{x} z, \quad u_{y}=-\left(R z_{y}-R_{y} z\right) .
$$

Furthermore, for $w=u / R$, we see that

$$
w_{x y}=M\left(\frac{1}{R}\right) w
$$

and

$$
(R w)_{x}=R^{2}\left(\frac{z}{R}\right)_{x}, \quad(R w)_{y}=-R^{2}\left(\frac{z}{R}\right)_{y} .
$$

Hence, $w$ is defined by the integral

$$
w=\frac{1}{R} \int R^{2}\left\{\left(\frac{z}{R}\right)_{x} d x-\left(\frac{z}{R}\right)_{y} d y\right\} .
$$

The transformation of any solution $z$ of (5.9) to a solution $w$ of (5.10) is called a Moutard transformation. 
Now let $z$ be any solution of the system (5.6) with parameter $m=a$ and choose a solution $R$ of the same system with parameter $m=b$. Assuming $a \neq b$, we define a new function $w$ by

$$
w=z-\frac{2 a(\log R)_{x}}{(a-b) h} z_{y}+\frac{2 b(\log R)_{y}}{(a-b) h} z_{x} .
$$

Then computation shows that

$$
(R w)_{x}=\frac{a+b}{a-b}\left(R z_{x}-R_{x} z\right), \quad(R w)_{y}=-\frac{a+b}{a-b}\left(R z_{y}-R_{y} z\right),
$$

which implies that $w$ is a Moutard transformation of $z$, up to a constant multiple. Furthermore, we can see that $w$ satisfies the system (5.6) with the same constant $m$ and with $h-2(\log R)_{x y}$ as the new $h$. Therefore, we have the following theorem due to Tzitzeica.

THEOREM 5.10. Let $z$ be an affine sphere and define a new surface $w$ by (5.11). Then:

(1) the surface $w$ is again an affine sphere and the associated system is determined by the potential $h-2(\log R)_{x y}$ for $h$ and the same parameter $m=a$ as for $z$;

(2) the congruence made by lines joining $z(x, y)$ and $w(x, y)$ is a $W$-congruence.

Part (2) follows from the fact that the conformal structure of both surfaces is conformal to $d x d y$. We remark that the potential $\bar{h}=h-2(\log R)_{x y}$ satisfies the equation $(\log \bar{h})_{x y}=\bar{h}-1 / \bar{h}^{2}$.

Remark 5.11. A reference for affine differential geometry is [NS94]. Fundamental references for Tzitzeica's work are [Tz07, Tz24]. We refer the reader to Section 13 for a generalization of Theorem 5.10, and to [RS02] for the Tzitzeica transformation. We also refer the reader to [BS99]. The article [Dem20] is a good reference for the Moutard transformation.

\section{Line congruences II}

In Section 3 we defined the notion of line congruence and explained a geometrical characterization of $\mathrm{W}$-congruence. This section aims at continuing the consideration in a slightly different manner, using the moving frame method and discussing the relation with a differential system. 

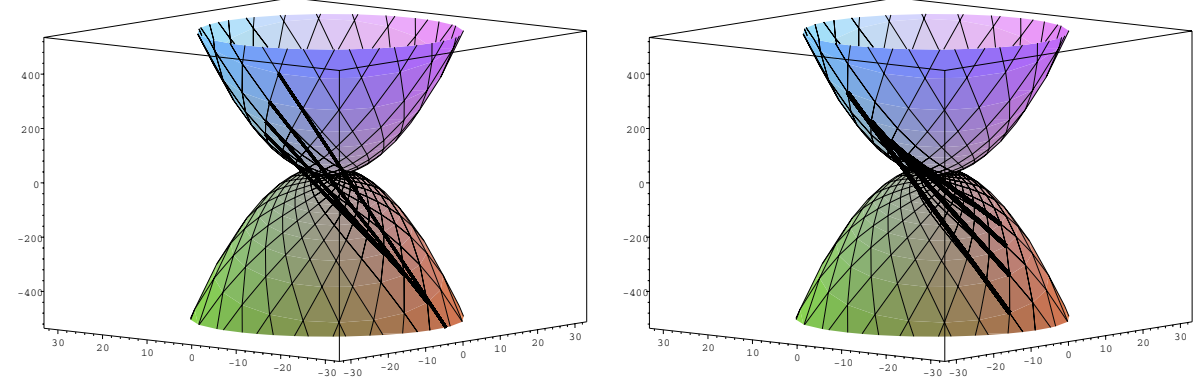

FIGURE 1. Line congruence between two paraboloids.

\subsection{The Weingarten invariant $W$}

Definition 6.1. A projective frame $e=\left\{e_{1}, e_{2}, e_{3}, e_{4}\right\}$ in $\mathbb{P}^{3}$ defined along an immersed surface $M^{2}$ is said to belong to a line congruence if the connection form $\omega$, defined by $d e_{i}=\sum_{j} \omega_{i}^{j} e_{j}$, satisfies the condition

$$
\omega_{1}^{4}=\omega_{2}^{3}=0, \quad \omega_{1}^{3} \text { and } \omega_{2}^{4} \text { are linearly independent. }
$$

We hereafter use the notation $\omega^{1}=\omega_{1}^{3}$ and $\omega^{2}=\omega_{2}^{4}$.

A geometric interpretation is the following. The vector $e_{1}$ is an immersion of $M$ and defines a surface $S_{1}$, and $e_{2}$ defines a second surface $S_{2}$; the set of lines joining two points $e_{1}$ and $e_{2}$ is a line congruence in the sense defined in Section 3. Figure 1 shows a line congruence between two paraboloids. The tangent lines to the parameter curves of one of two surfaces are tangent to the other surface.

As we can see $e_{1} \wedge e_{2} \wedge d e_{1} \wedge d e_{2}=\omega^{1} \cdot \omega^{2} e_{1} \wedge e_{2} \wedge e_{3} \wedge e_{4}$ by definition (remark that the wedge product is made relative to vectors and the product of differential forms is a symmetric product), the ruled surface given as the union of lines through the integral curve of the equation $\omega^{1}=0$ is developable. The same is true for $\omega^{2}=0$. Hence, the vectors $e_{1}$ and $e_{2}$ describe focal surfaces of the congruence.

Two frames belonging to the same line congruence are related by a matrix of the form

$$
g=\left(\begin{array}{llll}
\lambda & \beta & 0 & 0 \\
\gamma & \mu & 0 & 0 \\
p & q & \rho & 0 \\
r & s & 0 & \sigma
\end{array}\right)
$$


which acts on the frame from the left. Such matrices form a group, say $G$, and the set of such frames define a bundle over $M$ with $G$ the fiber group.

Let us see what the first condition of (6.1) implies. From $d \omega_{1}^{4}=0$, we have $\omega_{1}^{2} \wedge \omega_{2}^{4}+\omega_{1}^{3} \wedge \omega_{3}^{4}=0$. Hence, if we set

$$
\omega_{1}^{2}=h_{11} \omega^{1}+h_{12} \omega^{2}, \quad \omega_{3}^{4}=h_{31} \omega^{1}+h_{32} \omega^{2},
$$

then $h_{11}+h_{32}=0$. From $d \omega_{2}^{3}=0$, we have $\omega_{2}^{1} \wedge \omega_{1}^{3}+\omega_{2}^{4} \wedge \omega_{4}^{3}=0$ and we can set

$$
\omega_{2}^{1}=h_{21} \omega^{1}+h_{22} \omega^{2}, \quad \omega_{4}^{3}=h_{41} \omega^{1}+h_{42} \omega^{2},
$$

so that $h_{22}+h_{41}=0$.

LEMMA 6.2. There always exists a frame satisfying $h_{11}=h_{32}=h_{22}=h_{41}=0$. For such a frame, we have

$$
\omega_{1}^{2}=h_{12} \omega^{2}, \quad \omega_{3}^{4}=h_{31} \omega^{1}, \quad \omega_{2}^{1}=h_{21} \omega^{1}, \quad \omega_{4}^{3}=h_{42} \omega^{2} .
$$

Proof. We define a new frame $\bar{e}=\left\{\bar{e}_{1}, \bar{e}_{2}, \bar{e}_{3}, \bar{e}_{4}\right\}$ by

$$
\begin{gathered}
\bar{e}_{1}=e_{1}, \\
\bar{e}_{2}=e_{2}, \\
\bar{e}_{3}=p e_{1}+q e_{2}+e_{3}, \\
\bar{e}_{4}=r e_{1}+s e_{2}+e_{4} .
\end{gathered}
$$

Then it is not difficult to see that

$$
\begin{gathered}
d \bar{e}_{1}=\left(\omega_{1}^{1}-p \omega_{1}^{3}\right) \bar{e}_{1}+\left(\omega_{1}^{2}-q \omega_{1}^{3}\right) \bar{e}_{2}+\omega_{1}^{3} \bar{e}_{3}, \\
d \bar{e}_{2}=\left(\omega_{2}^{1}-r \omega_{2}^{4}\right) \bar{e}_{1}+\left(\omega_{2}^{2}-s \omega_{2}^{4}\right) \bar{e}_{2}+\omega_{2}^{4} \bar{e}_{4}, \\
d \bar{e}_{3} \equiv\left(\omega_{3}^{4}+q \omega_{2}^{4}\right) \bar{e}_{4} \quad\left(\bmod \bar{e}_{1}, \bar{e}_{2}, \bar{e}_{3}\right), \\
d \bar{e}_{4} \equiv\left(\omega_{4}^{3}+r \omega_{1}^{3}\right) \bar{e}_{3} \quad\left(\bmod \bar{e}_{1}, \bar{e}_{2}, \bar{e}_{4}\right) .
\end{gathered}
$$

This implies that the connection form $\bar{\omega}$ corresponding to $\bar{e}$ has an expression of the form

$$
\begin{aligned}
& \bar{\omega}_{1}^{2}=\omega_{1}^{2}-q \omega^{1}=\left(h_{11}-q\right) \omega^{1}+h_{12} \omega^{2}, \\
& \bar{\omega}_{2}^{1}=\omega_{2}^{1}-r \omega^{2}=h_{21} \omega^{1}+\left(h_{22}-r\right) \omega^{2}, \\
& \bar{\omega}_{3}^{4}=\omega_{3}^{4}+q \omega^{2}=h_{31} \omega^{1}+\left(h_{32}+q\right) \omega^{2}, \\
& \bar{\omega}_{4}^{3}=\omega_{4}^{3}+r \omega^{1}=\left(h_{41}+r\right) \omega^{1}+h_{42} \omega^{2} .
\end{aligned}
$$

Hence, the result follows, because $h_{11}+h_{32}=0$ and $h_{22}+h_{41}=0$. 
We now assume that the frame satisfies (6.2). Along the surface $S_{1}$, the projective frame in the previous section is given by $\left\{e_{1}, e_{2}, e_{3}, e_{4}\right\}$; hence, the induced conformal structure, which we now denote by $\phi_{1}$, is given by

$$
\phi_{1}=\omega_{1}^{2} \cdot \omega_{2}^{4}+\omega_{1}^{3} \cdot \omega_{3}^{4}=h_{31} \omega^{1} \omega^{1}+h_{12} \omega^{2} \omega^{2} .
$$

Similarly, for the surface $S_{2}$, we get the conformal structure

$$
\phi_{2}=h_{21} \omega^{1} \omega^{1}+h_{42} \omega^{2} \omega^{2} .
$$

Definition 6.3. We call $W=h_{12} h_{21}-h_{31} h_{42}$ the Weingarten invariant of the line congruence. This coincides with the definition of $W$ given in (3.7); see Section 6.3.

\subsection{Covariance of frames}

We now consider how frames satisfying the condition (6.2) can vary. Let $e$ and $\tilde{e}$ be such frames and let $g$ be the connecting matrix such that $\tilde{e}=g e$ :

$$
g=\left(\begin{array}{cc}
A & 0 \\
P & \Lambda
\end{array}\right) ; \quad A=\left(\begin{array}{cc}
\lambda & \beta \\
\gamma & \mu
\end{array}\right), \quad \Lambda=\left(\begin{array}{cc}
\rho & 0 \\
0 & \sigma
\end{array}\right), \quad P=\left(\begin{array}{ll}
p & q \\
r & s
\end{array}\right) .
$$

Then the connection forms are $\omega$ above and $\tilde{\omega}=d g \cdot g^{-1}+g \omega g^{-1}$. We denote components of $\omega$ by

$$
\omega=\left(\begin{array}{ll}
\omega_{1} & \omega_{2} \\
\omega_{3} & \omega_{4}
\end{array}\right)
$$

where $\omega_{1}, \ldots, \omega_{4}$ are $2 \times 2$-matrix-valued 1 -forms. The $\tilde{\omega}$ is also decomposed similarly. Computation shows that

$$
\begin{gathered}
d g \cdot g^{-1}=\left(\begin{array}{cc}
d A \cdot A^{-1} & 0 \\
d P \cdot A^{-1}-d \Lambda \cdot \Lambda^{-1} P A^{-1} & d \Lambda \cdot \Lambda^{-1}
\end{array}\right), \\
g \omega g^{-1}=\left(\begin{array}{cc}
A \omega_{1} A^{-1}-A \omega_{2} \Lambda^{-1} P A^{-1} & A \omega_{2} \Lambda^{-1} \\
P \omega_{1} A^{-1}+\Lambda \omega_{3} A^{-1}-P \omega_{2} \Lambda^{-1} p A^{-1} & P \omega_{2} \Lambda^{-1} \\
-\Lambda \omega_{4} \Lambda^{-1} P A^{-1} & +\Lambda \omega_{4} \Lambda^{-1}
\end{array}\right) .
\end{gathered}
$$

Thus, we see that

$$
\tilde{\omega}_{2}=A \omega_{2} \Lambda^{-1}=\left(\begin{array}{ll}
\lambda & \beta \\
\gamma & \mu
\end{array}\right)\left(\begin{array}{cc}
\rho^{-1} \omega_{1}^{3} & 0 \\
0 & \sigma^{-1} \omega_{2}^{4}
\end{array}\right) .
$$

In order that this has a form $\left(\begin{array}{cc}\tilde{\omega}_{1}^{3} & 0 \\ 0 & \tilde{\omega}_{2}^{4}\end{array}\right)$, it is necessary that $\beta=\gamma=0$. In this case,

$$
\left\{\begin{array}{l}
\tilde{e}_{1}=\lambda e_{1} \\
\tilde{e}_{2}=\mu e_{2}
\end{array} \quad \text { where } \quad A=\left(\begin{array}{ll}
\lambda & 0 \\
0 & \mu
\end{array}\right) .\right.
$$


We then have

$$
\tilde{\omega}_{1}^{3}=\lambda \rho^{-1} \omega_{1}^{3}, \quad \tilde{\omega}_{2}^{4}=\mu \sigma^{-1} \omega_{2}^{4} .
$$

For the component $\tilde{\omega}_{1}$, we get

$$
\begin{aligned}
\tilde{\omega}_{1}= & A \omega_{1} A^{-1}-A \omega_{2} \Lambda^{-1} p A^{-1}+d A \cdot A^{-1} \\
= & \left(\begin{array}{cc}
\omega_{1}^{1} & \mu^{-1} \lambda \omega_{1}^{2} \\
\lambda^{-1} \mu \omega_{2}^{1} & \omega_{2}^{2}
\end{array}\right)-\left(\begin{array}{cc}
\tilde{\omega}_{1}^{3} & 0 \\
0 & \tilde{\omega}_{2}^{4}
\end{array}\right)\left(\begin{array}{cc}
\lambda^{-1} p & \mu^{-1} q \\
\lambda^{-1} r & \mu^{-1} s
\end{array}\right) \\
& +\left(\begin{array}{cc}
d \log \lambda & 0 \\
0 & d \log \mu
\end{array}\right) .
\end{aligned}
$$

Hence,

$$
\tilde{\omega}_{1}^{2}=\lambda \mu^{-1}\left(\omega_{1}^{2}-\rho^{-1} q \omega_{1}^{3}\right), \quad \tilde{\omega}_{2}^{1}=\lambda^{-1} \mu\left(\omega_{2}^{1}-\sigma^{-1} r \omega_{1}^{3}\right) .
$$

Similarly,

$$
\begin{aligned}
\tilde{\omega}_{4}= & P \omega_{2} \Lambda^{-1}+\Lambda \omega_{4} \Lambda^{-1}+d \Lambda \cdot \Lambda^{-1} \\
= & \left(\begin{array}{ll}
p \omega_{1}^{3} & q \omega_{2}^{4} \\
r \omega_{1}^{3} & s \omega_{2}^{4}
\end{array}\right)\left(\begin{array}{ll}
\rho^{-1} & \\
& \sigma^{-1}
\end{array}\right)+\left(\begin{array}{ll}
\rho \omega_{3}^{3} & \rho \omega_{3}^{4} \\
\sigma \omega_{4}^{3} & \sigma \omega_{4}^{4}
\end{array}\right)\left(\begin{array}{cc}
\rho^{-1} & \\
& \sigma^{-1}
\end{array}\right) \\
& +\left(\begin{array}{cc}
d \log \rho & 0 \\
0 & d \log \sigma
\end{array}\right) .
\end{aligned}
$$

Hence,

$$
\begin{aligned}
& \tilde{\omega}_{3}^{3}=\rho^{-1}\left(p \omega_{1}^{3}+\rho \omega_{3}^{3}\right)+d \log \rho, \quad \tilde{\omega}_{3}^{4}=\sigma^{-1}\left(q \omega_{2}^{4}+\rho \omega_{3}^{4}\right), \\
& \tilde{\omega}_{4}^{3}=\rho^{-1}\left(r \omega_{1}^{3}+\sigma \omega_{4}^{3}\right), \quad \tilde{\omega}_{4}^{4}=\sigma^{-1}\left(s \omega_{2}^{4}+\sigma \omega_{4}^{4}\right)+d \log \sigma .
\end{aligned}
$$

We define $\tilde{h}_{i j}$ and $\tilde{\phi}_{i}$ for $\tilde{\omega}$ similarly as was done for $\omega$. Then the formulas above show the following:

$$
\begin{gathered}
\tilde{h}_{11}=\rho \mu^{-1} h_{11}-\mu^{-1} q, \quad \tilde{h}_{21}=\lambda^{-2} \rho \mu h_{21}, \\
\tilde{h}_{12}=\sigma \lambda \mu^{-2} h_{12}, \quad \tilde{h}_{22}=\sigma \lambda^{-1} h_{22}-\lambda^{-1} r, \\
\tilde{h}_{31}=\lambda^{-1} \sigma^{-1} \rho^{2} h_{31}, \quad \tilde{h}_{41}=\lambda^{-1} \sigma h_{41}+\lambda^{-1} r, \\
\tilde{h}_{32}=\mu^{-1} \rho h_{32}+\mu^{-1} q, \quad \tilde{h}_{42}=\rho^{-1} \mu^{-1} \sigma^{2} h_{42},
\end{gathered}
$$

and

$$
\tilde{\phi}_{1}=\lambda \sigma^{-1} \phi_{1}, \quad \tilde{\phi}_{2}=\rho^{-1} \mu \phi_{2} .
$$

Summarizing the above considerations, we have the following covariance relation between normalized frames. 
LEMMA 6.4. The change of frames keeping the condition $h_{11}=h_{32}=h_{22}=h_{41}$ $=0$ has the form

$$
g=\left(\begin{array}{llll}
\lambda & 0 & 0 & 0 \\
0 & \mu & 0 & 0 \\
p & 0 & \rho & 0 \\
0 & s & 0 & \sigma
\end{array}\right)
$$

The connection forms satisfy the following covariance relations:

$$
\begin{aligned}
\tilde{\omega}_{1}^{1}=\omega_{1}^{1}-\rho^{-1} p \omega^{1}+d \log \lambda, & \tilde{\omega}_{3}^{3}=\omega_{3}^{3}+\rho^{-1} p \omega^{1}+d \log \rho, \\
\tilde{\omega}_{1}^{2}=\mu^{-1} \lambda \omega_{1}^{2}, & \tilde{\omega}_{3}^{4}=\sigma^{-1} \rho \omega_{3}^{4}, \\
\tilde{\omega}_{2}^{1}=\lambda^{-1} \mu \omega_{2}^{1}, & \tilde{\omega}_{4}^{3}=\rho^{-1} \sigma \omega_{4}^{3}, \\
\tilde{\omega}_{2}^{2}=\omega_{2}^{2}-\sigma^{-1} s \omega^{2}+d \log \mu, & \tilde{\omega}_{4}^{4}=\omega_{4}^{4}+\sigma^{-1} s \omega^{2}+d \log \sigma .
\end{aligned}
$$

\subsection{Systems of differential equations of a line congruence}

We continue the considerations of Section 3.2. We have seen that the congruence $\{z, w\}$ with parameter $(x, y)$ is written by the system of equations

$$
\begin{gathered}
z_{y}=m w, \quad z_{x x}=a z+b w+c z_{x}+d w_{y}, \\
w_{x}=n z, \quad w_{y y}=a^{\prime} z+b^{\prime} w+c^{\prime} z_{x}+d^{\prime} w_{y} .
\end{gathered}
$$

So we get

$$
\begin{aligned}
& z_{x y}=m n z+m_{x} w, \quad w_{x y}=n_{y} z+m n w, \\
& z_{y y}=m_{y} w+m w_{y}, \quad w_{x x}=n_{x} z+n z_{x} .
\end{aligned}
$$

If we define a projective frame as follows:

$$
e_{1}=z, \quad e_{2}=w, \quad e_{3}=z_{x}, \quad e_{4}=w_{y},
$$

then the associated connection form is

$$
\omega=\left(\begin{array}{cccc}
0 & m d y & d x & 0 \\
n d x & 0 & 0 & d y \\
a d x+m n d y & b d x+m_{x} d y & c d x & d d x \\
a^{\prime} d y+n_{y} d x & b^{\prime} d y+m n d x & c^{\prime} d y & d^{\prime} d y
\end{array}\right) .
$$

In particular, we see that

$$
\begin{aligned}
& \omega^{1}=d x, \quad \omega^{2}=d y, \\
& h_{11}=0, \quad h_{12}=m, \quad h_{31}=d, \quad h_{32}=0 \text {, } \\
& h_{21}=n, \quad h_{22}=0, \quad h_{41}=0, \quad h_{42}=c^{\prime},
\end{aligned}
$$


and

$$
W=m n-c^{\prime} d .
$$

The last expression coincides with the definition of $W$ given in Section 3.2. The invariant quadratic forms are

$$
\phi_{1}=d(d x)^{2}+m(d y)^{2}, \quad \phi_{2}=n(d x)^{2}+c^{\prime}(d y)^{2} .
$$

Compare (3.4) and (3.5) with (6.7) and (6.8). In the following, we assume that $m n c^{\prime} d \neq 0$ (refer to (3.6)) so that both focal surfaces are non-degenerate; we say that such a line congruence is non-degenerate.

The integrability condition of the system is nothing but $d \omega=\omega \wedge \omega$. A calculation shows that

$$
\omega \wedge \omega=\left(\begin{array}{cccc}
0 & m_{x} & 0 & 0 \\
-n_{y} & 0 & 0 & 0 \\
(m n)_{x}-a_{y} & m_{x x}-b_{y} & -c_{y} & -d_{y} \\
a_{x}^{\prime}-n_{y y} & b_{x}^{\prime}-(m n)_{y} & c_{x}^{\prime} & d_{x}^{\prime}
\end{array}\right) d x \wedge d y .
$$

Hence, the integrability condition of the system (6.4) consists of the following eight equations:

$$
\begin{gathered}
(m n)_{x}+n m_{x}-a_{y}-c m n-a^{\prime} d=0, \\
m_{x x}-b_{y}-a m-c m_{x}-b^{\prime} d=0, \\
c_{y}-m n+c^{\prime} d=0, \\
d_{y}+b+d d^{\prime}=0, \\
(m n)_{y}+m n_{y}-b_{x}^{\prime}-b c^{\prime}-m n d^{\prime}=0, \\
n_{y y}-a_{x}^{\prime}-b^{\prime} n-d^{\prime} n_{y}-a c^{\prime}=0, \\
d_{x}^{\prime}-m n+c^{\prime} d=0, \\
c_{x}^{\prime}+a^{\prime}+c c^{\prime}=0 .
\end{gathered}
$$

From the third and the seventh equations, we get $c_{y}=d_{x}^{\prime}$. This implies that there is a non-vanishing function $f$ so that

$$
c=-f_{x} / f, \quad d^{\prime}=-f_{y} / f .
$$

In particular,

$$
W=m n-c^{\prime} d=-(\log f)_{x y} .
$$

If we set

$$
\Delta=z \wedge w \wedge z_{x} \wedge w_{y},
$$


then a simple calculation shows

$$
\Delta_{x}=c \Delta, \quad \Delta_{y}=d^{\prime} \Delta .
$$

Namely, we have $\Delta=1 / f$ from (6.10), up to a constant multiple.

The system (6.4) is not uniquely determined by the given congruence. It has some freedom of choice. One is a change of parameters

$$
\text { (1) }(x, y) \longmapsto(\bar{x}=X(x), \bar{y}=Y(y)),
$$

and the other is a change of coordinates in $\mathbb{P}^{3}$

$$
\text { (2) }(z, w) \longmapsto(\bar{z}=z / \lambda(x), \bar{w}=w / \mu(y)) \text {. }
$$

We look for dependence of coefficients separately for the two cases. By the change (1), the system is written as follows:

$$
\begin{gathered}
z \bar{y}=\frac{m}{Y_{y}} w, \quad z \overline{x x}=\frac{1}{X_{x}^{2}}\left(a z+b w+\left(c X_{x}-X_{x x}\right) z_{\bar{x}}+d Y_{y} w_{\bar{y}}\right), \\
w_{\bar{x}}=\frac{n}{X_{x}} z, \quad w_{\overline{y y}}=\frac{1}{Y_{y}^{2}}\left(a^{\prime} z+b^{\prime} w+c^{\prime} X_{x} z \bar{x}+\left(d^{\prime} Y_{y}-Y_{y y}\right) w_{\bar{y}}\right) .
\end{gathered}
$$

In particular, the Weingarten invariant $\bar{W}$ of the new system is

$$
\bar{W}=\frac{1}{X_{x} Y_{y}} W .
$$

Under the change (2), the new system relative to $(\bar{z}, \bar{w})$ is

$$
\begin{gathered}
\bar{z}_{y}=\frac{\mu}{\lambda} m \bar{w}, \bar{w}_{x}=\frac{\lambda}{\mu} n \bar{z}, \\
\bar{z}_{x x}=\left(a+\frac{\lambda_{x}}{\lambda} c-\frac{\lambda_{x x}}{\lambda}\right) \bar{z}+\left(\frac{\mu}{\lambda} b+\frac{\mu_{y}}{\lambda} d\right) \bar{w}+\left(c-\frac{2 \lambda_{x}}{\lambda}\right) \bar{z}_{x}+\frac{\mu}{\lambda} d \bar{w}_{y}, \\
\bar{w}_{y y}=\left(\frac{\lambda}{\mu} a^{\prime}+\frac{\lambda_{x}}{\mu} c^{\prime}\right) \bar{z}+\left(b^{\prime}+\frac{\mu_{y}}{\mu} d^{\prime}-\frac{\mu_{y y}}{\mu}\right) \bar{w}+\frac{\lambda}{\mu} c^{\prime} \bar{z}_{x}+\left(d^{\prime}-\frac{2 \mu_{y}}{\mu}\right) \bar{w}_{y} .
\end{gathered}
$$

It is easy to see that the Weingarten invariant is unchanged. As $c_{y}=W$ and $d_{x}^{\prime}=-W$, we can solve $c-2 \lambda_{x} / \lambda=0$ and $d^{\prime}-2 \mu_{y} / \mu=0$ in the case $W=0$; namely, we have the following.

COROLLARY 6.5. If $W=0$, then the system can be reduced to the case $c=d^{\prime}=0$.

By composing the above two kinds of changes, we get the following. 
LEMMA 6.6. By the change of variables

$$
(x, y ; z, w) \longmapsto(\bar{x}=X(x), \bar{y}=Y(y) ; \bar{z}=z / \lambda(x), \bar{w}=w / \mu(y)),
$$

the coefficients $m, n, c^{\prime}$ and $d$ are changed as follows:

$$
m \longmapsto \frac{\mu}{\lambda Y_{y}} m, \quad n \longmapsto \frac{\lambda}{\mu X_{x}} n, \quad c^{\prime} \longmapsto \frac{\lambda X_{x}}{\mu Y_{y}^{2}} c^{\prime}, \quad d \longmapsto \frac{\mu Y_{y}}{\lambda X_{x}^{2}} d .
$$

\subsection{The dual of a line congruence}

Let $\{z, w\}$ be a line congruence. We denote by $T_{z}$ (respectively $T_{w}$ ) the tangent space of the surface $z$ (respectively $w$ ). Each line of the congruence is represented by the intersection $T_{z} \cap T_{w}$. The dual of the plane $T_{z}$, which we identify with the vector $z \wedge z_{x} \wedge z_{y}$, describes a surface in the dual projective space. Similarly, we have the surface given by $w \wedge w_{x} \wedge w_{y}$. Thus, we get a congruence consisting of lines connecting two surfaces that are points in the dual projective space. We call this congruence the dual of a given congruence, or more briefly the dual congruence. We now give the system of the dual congruence.

As $z \wedge z_{x} \wedge z_{y}=m z \wedge z_{x} \wedge w$ and $w \wedge w_{x} \wedge w_{y}=n w \wedge z \wedge w_{y}$, and as we have assumed that $m \neq 0$ and $n \neq 0$, the dual congruence is well-defined. Then the dual image is described by two vectors

$$
\begin{gathered}
U=\frac{1}{n \Delta} w \wedge w_{x} \wedge w_{y}=-\frac{1}{\Delta} z \wedge w \wedge w_{y}, \\
V=\frac{1}{m \Delta} z \wedge z_{x} \wedge z_{y}=-\frac{1}{\Delta} z \wedge w \wedge z_{x} .
\end{gathered}
$$

Proposition 6.7. The system of the dual line congruence $\{U, V\}$ is the following:

$$
\begin{gathered}
U_{y}=c^{\prime} V, \quad U_{x x}=\left(a-c_{x}\right) U+\left(n d^{\prime}-n_{y}\right) V-c U_{x}+n V_{y}, \\
V_{x}=d U, \quad V_{y y}=\left(c m-m_{x}\right) U+\left(b^{\prime}-d_{y}^{\prime}\right) V+m U_{x}-d^{\prime} V_{y} .
\end{gathered}
$$

The Weingarten invariant $W^{d}$ of the dual congruence is

$$
W^{d}=c^{\prime} d-m n=-W .
$$

COROLLARY 6.8. If the dual congruence is projectively equivalent to the original congruence, then the Weingarten invariant vanishes.

In this case, because of Corollary 6.5, the system can be reduced to

$$
\begin{gathered}
U_{y}=c^{\prime} V, \quad U_{x x}=a U-n_{y} V+n V_{y}, \\
V_{x}=d U, \quad V_{y y}=-m_{x} U+b^{\prime} V+m U_{x} .
\end{gathered}
$$




\section{Linear complexes}

Each line of a line congruence can be regarded a point of $\mathbb{P}^{5}$ through the Plücker embedding, and thus any line congruence can be regarded as a surface in $\mathbb{P}^{5}$ contained in the quadratic hypersurface determined by the Plücker relation. In this section, we consider such a line congruence in the case that the image surface is contained in a hyperplane.

\subsection{Linear complexes}

We say that a three-dimensional family of lines is a linear complex when its Plücker image into $\mathbb{P}^{5}$ lies in a hyperplane.

If $y=\left[y^{1}, y^{2}, y^{3}, y^{4}\right]$ and $z=\left[z^{1}, z^{2}, z^{3}, z^{4}\right]$ are two points on a line, the Plücker image of the line is the vector $y \wedge z$. The homogeneous coordinates of $y \wedge z$ are $\left[\tau_{12}, \tau_{13}, \tau_{14}, \tau_{23}, \tau_{42}, \tau_{34}\right]$, where $\tau_{i j}=y^{i} z^{j}-y^{j} z^{i}$. When they satisfy a linear relation

$$
a_{34} \tau_{12}+a_{42} \tau_{13}+a_{23} \tau_{14}+a_{14} \tau_{23}+a_{13} \tau_{42}+a_{12} \tau_{34}=0,
$$

we have an associated vector $\xi=\left[\xi_{1}, \xi_{2}, \xi_{3}, \xi_{4}\right]$ given by

$$
\xi=\left(\begin{array}{cccc}
0 & -a_{34} & -a_{42} & -a_{23} \\
a_{34} & 0 & -a_{14} & a_{13} \\
a_{42} & a_{14} & 0 & -a_{12} \\
a_{23} & -a_{13} & a_{12} & 0
\end{array}\right)\left(\begin{array}{l}
y^{1} \\
y^{2} \\
y^{3} \\
y^{4}
\end{array}\right)
$$

where $\xi$ is also regarded as a column vector. Then, we see that the linear relation is written as $\sum \xi_{i} z^{i}=0$. Hence, $\xi$ can be regarded as the vector defining a hyperplane, on which lies the point $z$. By definition, the point $y$ itself lies on the hyperplane. This means that, when given a linear complex, for any point $y$, we can associate a hyperplane through $y$ so that the lines on this hyperplane through $y$ altogether form the linear complex. The correspondence of $y$ to $\xi$ is a linear transformation of the space $\mathbb{P}^{3}$ to its dual space. Conversely, such a transformation of the above form defines a linear complex.

\subsection{The Plücker image of a line congruence}

The Plücker image of a line congruence $\{z, w\}$ is identified with

$$
\xi=z \wedge w .
$$


The mapping $(x, y) \mapsto \xi(x, y)$ defines generally a surface in $\mathbb{P}^{5}$. As we know any surface in $\mathbb{P}^{5}$ can be described by a system of third-order differential equations, we compute the associated system for $\xi$ here.

For simplicity, we set

$$
\eta=z \wedge z_{x} \text { and } \zeta=w \wedge w_{y} .
$$

By using (6.4), we see that

$$
\xi_{x}=z_{x} \wedge w, \quad \xi_{y}=z \wedge w_{y},
$$

and, by (6.4) and (6.5),

$$
\begin{gathered}
\xi_{x x}=a \xi+c \xi_{x}-n \eta-d \zeta, \\
\xi_{x y}=m n \xi+z_{x} \wedge w_{y}, \\
\xi_{y y}=b^{\prime} \xi+d^{\prime} \xi_{y}+c^{\prime} \eta+m \zeta .
\end{gathered}
$$

Then differentiating once more, we get third-order relations:

$$
\begin{gathered}
\xi_{x x x}=c \xi_{x x}+\left(a+c_{x}\right) \xi_{x}-2 d n \xi_{y}+\left(a_{x}-b n+d n_{y}\right) \xi-\left(c n+n_{x}\right) \eta-d_{x} \zeta, \\
\xi_{x x y}=c \xi_{x y}+2 m n \xi_{x}+a \xi_{y}+\left((m n)_{x}-c m n\right) \xi-n_{y} \eta+b \zeta, \\
\xi_{x y y}=d^{\prime} \xi_{x y}+b^{\prime} \xi_{x}+2 m n \xi_{y}+\left((m n)_{y}-m n d^{\prime}\right) \xi-a^{\prime} \eta+m_{x} \zeta, \\
\xi_{y y y}=d^{\prime} \xi_{y y}-2 c^{\prime} m \xi_{x}+\left(b^{\prime}+d_{y}^{\prime}\right) \xi_{y}+\left(b_{y}^{\prime}-a^{\prime} m+c^{\prime} m_{x}\right) \xi+c_{y}^{\prime} \eta+\left(m_{y}+m d^{\prime}\right) \zeta .
\end{gathered}
$$

From the first and third relations of (7.1), we have

$$
\begin{aligned}
& W \eta=-m\left(\xi_{x x}-c \xi_{x}-a \xi\right)-d\left(\xi_{y y}-d^{\prime} \xi_{y}-b^{\prime} \xi\right), \\
& W \zeta=c^{\prime}\left(\xi_{x x}-c \xi_{x}-a \xi\right)+n\left(\xi_{y y}-d^{\prime} \xi_{y}-b^{\prime} \xi\right),
\end{aligned}
$$

where $W=m n-c^{\prime} d$. Hence, if the Weingarten invariant does not vanish, the equations in (7.2) can be written in closed forms depending only on $\xi$ and thus the system associated with $\xi$ is derived. For later use, we list the system up to secondorder terms:

$$
\begin{gathered}
\xi_{x x x}=\left(c+\frac{c m n+m n_{x}-c^{\prime} d_{x}}{W}\right) \xi_{x x}+\frac{\left(c n+n_{x}\right) d-n d_{x}}{W} \xi_{y y} \quad\left(\bmod \xi_{x}, \xi_{y}, \xi\right), \\
\xi_{x x y}=\frac{m n_{y}+b c^{\prime}}{W} \xi_{x x}+c \xi_{x y}+\frac{d n_{y}+b n}{W} \xi_{y y} \quad\left(\bmod \xi_{x}, \xi_{y}, \xi\right), \\
\xi_{x y y}=\frac{a^{\prime} m+c^{\prime} m_{x}}{W} \xi_{x x}+d^{\prime} \xi_{x y}+\frac{a^{\prime} d+m_{x} n}{W} \xi_{y y} \quad\left(\bmod \xi_{x}, \xi_{y}, \xi\right), \\
\xi_{y y y}=\frac{\left(m_{y}+m d^{\prime}\right) c^{\prime}-c_{y}^{\prime} m}{W} \xi_{x x}+\left(d^{\prime}+\frac{\left(m_{y}+m d^{\prime}\right) n-c_{y}^{\prime} d}{W}\right) \xi_{y y} \quad\left(\bmod \xi_{x}, \xi_{y}, \xi\right) .
\end{gathered}
$$


If the Weingarten invariant vanishes, $\xi$ must satisfy a second-order differential equation

$$
m\left(\xi_{x x}-c \xi_{x}-a \xi\right)+d\left(\xi_{y y}-d^{\prime} \xi_{y}-b^{\prime} \xi\right)=0,
$$

which shows Theorem 3.3 again.

Given a line congruence $\{z, w\}$, we next construct the following sets of lines: $A_{1}(x, y)=$ the set of lines joining $z(x, y)$ and any point on the line $\overline{w(x, y) w_{y}(x, y)}$,

$$
L_{1}=\bigcup_{x, y} A_{1}(x, y)
$$

$A_{2}(x, y)=$ the set of lines joining $w(x, y)$ and any point on the line $\overline{z(x, y) z_{x}(x, y)}$,

$$
L_{2}=\bigcup_{x, y} A_{2}(x, y) \text {. }
$$

For a line congruence $\{z, w\}$, the set of vectors $\left\{z, w, z_{x}, w_{y}\right\}$ defines a projective frame. We associate to it a moving frame in $\mathbb{P}^{5}$ by defining

$$
\begin{gathered}
\xi_{12}=z \wedge w, \quad \xi_{13}=z \wedge z_{x}, \quad \xi_{14}=z \wedge w_{y}, \\
\xi_{23}=w \wedge z_{x}, \quad \xi_{24}=w \wedge w_{y}, \quad \xi_{34}=z_{x} \wedge w_{y} .
\end{gathered}
$$

The Plücker coordinates $p_{i j}$ of any point of $p \in \mathbb{P}^{5}$ relative to the frame $\left\{\xi_{i j}\right\}$ are defined by setting

$$
p=p_{12} \xi_{12}+p_{13} \xi_{13}+p_{14} \xi_{14}+p_{23} \xi_{23}+p_{24} \xi_{24}+p_{34} \xi_{34}
$$

The Pluc̈ker image of a line in $L_{1}$ is given by

$$
\eta=z \wedge\left(w+t w_{y}\right),
$$

where $t$ is a parameter. We regard $\eta$ as a point in $\mathbb{P}^{5}$ with three parameters $x, y$ and $t$. By a computation we have

$$
\begin{gathered}
\eta_{x}=z_{x} \wedge w+t z \wedge w_{y}+t m n z \wedge w, \\
\eta_{y}=t m w \wedge w_{y}+\left(1+t d^{\prime}\right) z \wedge w_{y}+t b^{\prime} z \wedge w+t c^{\prime} z \wedge z_{x}, \\
\eta_{t}=z \wedge w .
\end{gathered}
$$

Hence, the coordinates of these vectors are written as follows:

\begin{tabular}{ccccccc}
\hline & $p_{12}$ & $p_{13}$ & $p_{14}$ & $p_{23}$ & $p_{24}$ & $p_{34}$ \\
\hline$\eta$ & 1 & 0 & $t$ & 0 & 0 & 0 \\
$\eta_{x}$ & $t m n$ & 0 & 0 & -1 & 0 & $t$ \\
$\eta_{y}$ & $t b^{\prime}$ & $t c^{\prime}$ & $1+t d^{\prime}$ & 0 & $t m$ & 0 \\
$\eta_{t}$ & 0 & 0 & 1 & 0 & 0 & 0 \\
\hline
\end{tabular}


From this table of coordinates, we can see that the hyperplane

$$
m p_{13}-c^{\prime} p_{24}=0
$$

is the unique hyperplane tangent to the set $L_{1}$ and including lines in $A_{1}(x, y)$ at each fixed value $(x, y)$. Similarly, for the set $L_{2}$, we get the hyperplane

$$
d p_{13}-n p_{24}=0 \text {. }
$$

These two hyperplanes coincide if and only if $W=0$.

\subsection{Line congruences belonging to a linear complex}

We next want to consider when the hyperplanes obtained in Section 7.2 do not depend on $(x, y)$; namely, when the line congruence belongs to a linear complex.

Let us set

$$
\zeta=m p_{13}-c^{\prime} p_{24}
$$

considered as a vector-valued function of $(x, y)$. The condition we need is

$$
\zeta_{x} \equiv 0 \quad \text { and } \quad \zeta_{y} \equiv 0 \quad(\bmod \zeta)
$$

By differentiating (7.3), we see that $\sum_{i<j}\left(d p_{i j} \xi_{i j}+p_{i j} d \xi_{i j}\right)=0$. If we write $d\left(\xi_{i j}\right)=(M d x+N d y)\left(\xi_{i j}\right)$, where $M$ and $N$ are $(6 \times 6)$-matrices, we have

$$
p_{i j x}=-\sum_{k \ell} p_{k \ell} M_{i j}^{k \ell} \quad \text { and } \quad p_{i j y}=-\sum_{k \ell} p_{k \ell} N_{i j}^{k \ell} .
$$

As

$$
\begin{gathered}
\xi_{12 x}=-\xi_{23}, \quad \xi_{13 x}=b \xi_{12}+c \xi_{13}+d \xi_{14}, \\
\xi_{14 x}=m n \xi_{12}, \quad \xi_{23 x}=-a \xi_{12}+n \xi_{13}+c \xi_{23}+d \xi_{24}, \\
\xi_{24 x}=-n_{y} \xi_{12}+n \xi_{14}, \\
\xi_{34 x}=-n_{y} \xi_{13}+a \xi_{14}-m n \xi_{23}+b \xi_{24}+c \xi_{34},
\end{gathered}
$$

we have

$$
M=\left(\begin{array}{cccccc}
0 & 0 & 0 & -1 & 0 & 0 \\
b & c & d & 0 & 0 & 0 \\
m n & 0 & 0 & 0 & 0 & 1 \\
-a & n & 0 & c & d & 0 \\
-n_{y} & 0 & n & 0 & 0 & 0 \\
0 & -n_{y} & a & -m n & b & c
\end{array}\right)
$$


Namely, we get

$$
p_{13 x}=-c p_{13}-n p_{23}+n_{y} p_{34}, \quad p_{24 x}=-d p_{23}-b p_{34} .
$$

Therefore, we get

$$
\zeta_{x}=\left(m_{x}-c m\right) p_{13}-\left(m n-c^{\prime} d\right) p_{23}-c_{x}^{\prime} p_{24}+\left(m n_{y}+b c^{\prime}\right) p_{34},
$$

and the condition that $\zeta_{x} \equiv 0(\bmod \zeta)$ is equal to

$$
W=m n-c^{\prime} d=0, \quad m n_{y}+b c^{\prime}=0, \quad\left|\begin{array}{cc}
m_{x}-c m & c_{x}^{\prime} \\
m & c^{\prime}
\end{array}\right|=0 .
$$

By a similar computation, we have the following:

$$
\begin{gathered}
\xi_{12 y}=\xi_{14}, \quad \xi_{13 y}=m_{x} \xi_{12}+m \xi_{23}, \\
\xi_{14 y}=b^{\prime} \xi_{12}+c^{\prime} \xi_{13}+d^{\prime} \xi_{14}+m \xi_{24}, \\
\xi_{23 y}=-m n \xi_{12}-\xi_{34} \\
\xi_{24 y}=-a^{\prime} \xi_{12}+c^{\prime} \xi_{23}+d^{\prime} \xi_{24}, \\
\xi_{34 y}=-a^{\prime} \xi_{13}+m n \xi_{14}-b^{\prime} \xi_{23}+m_{x} \xi_{24}+d^{\prime} \xi_{34},
\end{gathered}
$$

and

$$
N=\left(\begin{array}{cccccc}
0 & 0 & 1 & 0 & 0 & 0 \\
m_{x} & 0 & 0 & m & 0 & 0 \\
b^{\prime} & c^{\prime} & d^{\prime} & 0 & m & 0 \\
-m n & 0 & 0 & 0 & 0 & -1 \\
-a^{\prime} & 0 & 0 & c^{\prime} & d^{\prime} & 0 \\
0 & -a^{\prime} & m n & -b^{\prime} & m_{x} & d^{\prime}
\end{array}\right)
$$

Hence,

$$
p_{13 y}=-c^{\prime} p_{14}+a^{\prime} p_{34}, \quad p_{24 y}=m p_{14}-d^{\prime} p_{24}-m_{x} p_{34}
$$

Then we see

$$
\zeta_{y}=m_{y} p_{13}-\left(c_{y}^{\prime}-c^{\prime} d^{\prime}\right) p_{24}+\left(m a^{\prime}+c^{\prime} m_{x}\right) p_{34},
$$

and the condition that $\zeta_{y} \equiv 0(\bmod \zeta)$ is equivalent to

$$
m a^{\prime}+c^{\prime} m_{x}=0, \quad\left|\begin{array}{cc}
m_{y} & c_{y}^{\prime}-c^{\prime} d^{\prime} \\
m & c^{\prime}
\end{array}\right|=0 .
$$

As $W=0$ by (7.4), we need not examine the set $L_{2}$. Namely, the condition that a line congruence belongs to a linear complex is (7.4) and (7.5). 
THEOREM 7.1. A non-degenerate line congruence belongs to a linear complex if and only if the condition

$$
c=d^{\prime}=0, \quad c^{\prime}=m, \quad d=n, \quad b=-n_{y}, \quad a^{\prime}=-m_{x}
$$

is satisfied. The system (6.4) then reduces to

$$
\begin{gathered}
z_{y}=m w, \quad z_{x x}=a z-n_{y} w+n w_{y}, \\
w_{x}=n w, \quad w_{y y}=-m_{x} z+b^{\prime} w+m z_{x} .
\end{gathered}
$$

Proof. As $W=0$, we may assume that $c=0$ and $d^{\prime}=0$, by Corollary 6.5. Then the two determinants in (7.4) and (7.5) are

$$
\left|\begin{array}{cc}
m_{x} & c_{x}^{\prime} \\
m & c^{\prime}
\end{array}\right|=\left|\begin{array}{cc}
m_{y} & c_{y}^{\prime} \\
m & c^{\prime}
\end{array}\right|=0
$$

from which $c^{\prime} / m$ is seen to be a constant. By a frame change in Lemma 6.6, the quantity $c^{\prime} / m$ can be multiplied by $\lambda^{2} X_{x} / \mu^{2} Y_{y}$. Hence, we may assume that

$$
m=c^{\prime} \text {. }
$$

Note that by this change the condition $c=d^{\prime}=0$, i.e. the condition $\omega_{3}^{3}=\omega_{4}^{4}=0$ in view of (6.6), is preserved, by the formula (6.3) applied to the case where $p=s=0$. Thus, we have completed the proof.

Remark 7.2. Under the condition (7.6), the dual congruence is

$$
\begin{gathered}
U_{y}=m V, \quad U_{x x}=a U+b V+n V_{y}, \\
V_{x}=n U, \quad V_{y y}=a^{\prime} U+b^{\prime} V+m U_{x} .
\end{gathered}
$$

Hence, it is autoreciprocal; we refer the reader to (6.12).

Remark 7.3. The argument in this section relies on [W11]. We refer to Chern [Ch36] for some higher order invariants by which the family of quadratic complexes associated to a line congruence is described.

\section{Laplace transforms of a line congruence}

Let $\{z, w\}$ be a line congruence normalized as in (6.4). The point $w$ lies on the tangent line of the surface $z$ in the direction of the parameter $y$. This means that the line congruence is one of the tangent congruences of $z$ associated with parameter curves. Hence, the other line congruence determined by the parameter $x$ should be canonically associated to the given one. This section deals with this correspondence. 


\subsection{Laplace transforms of a line congruence}

Suppose that we are given a line congruence $\{z, w\}$, which we denote by $\Gamma_{0}$. Let us consider the tangent congruence $\left\{z_{x}, z\right\}$, for which the surface $z$ is by definition one of the focal surfaces. We want to find the other focal surface, which should have the form

$$
z_{1}=z_{x}+\alpha z
$$

The $\alpha$ is determined by supposing that

$$
z_{1 y} \equiv 0 \quad\left(\bmod z, z_{1}\right)
$$

As $z_{1 y}=\left(\alpha_{y}+m n\right) z+\left(\alpha m+m_{x}\right) w$ by (6.4), we must have $\alpha=-m_{x} / m$. In this case, we call the congruence $\Gamma_{1}=\left\{z_{1}, z\right\}$ the Laplace transform of $\Gamma_{0}$.

We now compute the system associated with $\Gamma_{1}$. Set $w_{1}=z / m$. Then

$$
\begin{gathered}
z_{1 y}=\left(m n-(\log m)_{x y}\right) m w_{1}, \\
z_{1 x}=\left(a-(\log m n)_{x x}\right) z+b w+\left(c-\frac{m_{x}}{m}\right) z_{x}+d w_{y}, \\
w_{1 x}=\frac{1}{m} z_{1}, \quad w_{1 y}=-\frac{m_{y}}{m^{2}} z+w .
\end{gathered}
$$

From these relations, we can compute the coefficients of the system describing the congruence $\left\{z_{1}, w_{1}\right\}$. Denoting the coefficients by attaching the subscript 1 , we get the following:

$$
\begin{aligned}
m_{1}= & m\left(m n-(\log m)_{x y}\right), \\
n_{1}= & 1 / m, \\
a_{1}= & a-\left(\log f m^{2}\right)_{x x}+(\log d / m)_{x}(\log f m)_{x}, \\
b_{1}= & m\left(a_{x}-a d_{x} / d+b n+d n_{y}-(\log m)_{x x x}\right)+(\log m)_{y}\left(b_{x}-b d_{x} / d+m n d\right) \\
& +m_{x}\left(a-\left(\log f m^{2}\right)_{x x}+(\log f m)_{x}(\log d / m)_{x}\right)-m(\log m)_{x x}(\log f m / d)_{x}, \\
c_{1}= & -(\log f m / d)_{x}, \\
d_{1}= & b_{x}-b d_{x} / d+d m n, \\
a_{1}^{\prime}= & (\log f m)_{x} / d, \\
b_{1}^{\prime}= & -(\log m)_{y y}-\left\{a m-m(\log m)_{x x}+b(\log m)_{y}-m_{x}(\log f m)_{x}\right\} / d, \\
c_{1}^{\prime}= & 1 / d, \\
d_{1}^{\prime}= & -b / d-(\log m)_{y},
\end{aligned}
$$

where $f$ was defined in (6.10). 
The transform in the inverse direction is $\Gamma_{-1}=\left\{z_{-1}, w_{-1}\right\}$ given by

$$
z_{-1}=\frac{w}{n}, \quad w_{-1}=w_{y}-\frac{n_{y}}{n} w .
$$

THEOREM 8.1. [Dem11, W15] If the first transform of a W-congruence is a $W$-congruence, then the same is true for all of its Laplace transforms.

Proof. As $W=-(\log f)_{x y}$ by $(6.11)$ and $W_{1}=m_{1} n_{1}-c_{1}^{\prime} d_{1}$ equals $-(\log m)_{x y}+$ $(\log d / f)_{x y}$ by (8.1) and by the identity $b=-d_{y}-d d^{\prime}$ in (6.9), we get

$$
W_{1}-W=(\log d)_{x y}-(\log m)_{x y} .
$$

Furthermore, we get

$$
\frac{m_{1}}{d_{1}}=\frac{m\left(m n-(\log m)_{x y}\right)}{d\left(m n-(\log d / f)_{x y}\right)} .
$$

Then, $W_{1}=W=0$ implies that $(\log f)_{x y}=0$ and $(\log d)_{x y}=(\log m)_{x y}$, which imply the identity $m_{1} / d_{1}=m / d$. This means that we can continue the process while preserving the identity.

Remark 8.2. We can define the sequence of Laplace transforms successively. $\mathrm{Su}[\mathrm{Su35}$, Su36a] and $\mathrm{Hu}[\mathrm{Hu93}]$ studied and showed interesting results for the case where the sequence is four-times periodic.

\subsection{Laplace transforms of a linear complex}

The property of belonging to a linear complex reflects a kind of degeneration of line congruences. Wilczynski considered the case where both $\Gamma_{0}$ and $\Gamma_{1}$ belong to linear complexes and derived the sinh-Gordon equation describing such congruences. We will reproduce his computation in this section. The number field in this section is the real field.

The condition that $\Gamma_{0}$ belongs to a linear complex was given in (7.6):

$$
c=d^{\prime}=0, \quad m=c^{\prime}, \quad n=d, \quad b=-n_{y}, \quad a^{\prime}=-m_{x} .
$$

In this case, the invariants of $\Gamma_{1}$ are

$$
m_{1}=m\left(m n-(\log m)_{x y}\right), \quad n_{1}=1 / m, \quad c_{1}^{\prime}=1 / n, \quad d_{1}=n\left(m n-(\log n)_{x y}\right)
$$

and the Weingarten invariant is $W_{1}=(\log (n / m))_{x y}$. As $W_{1}$ vanishes when $\Gamma_{1}$ belongs to a linear complex, we can write

$$
m / n=\alpha(x) \beta(y)
$$


for certain non-vanishing functions $\alpha$ and $\beta$. (Recall that we are assuming that $m \neq 0$ and $n \neq 0$.) On the other hand, a change of variables from $(x, y ; z, w)$ to $(\bar{x}, \bar{y}, \bar{z}, \bar{w})$ that preserves the condition (7.6) is subject to the condition

$$
\lambda^{2} X_{x}=\mu^{2} Y_{y}=\text { constant, }
$$

and $m / n$ is multiplied by $\mu^{2} X_{x} / \lambda^{2} Y_{y}$. Hence, we can assume

$$
m= \pm n \text {. }
$$

We have thus reduced the system to a simpler form with coefficients

$$
c=d^{\prime}=0, \quad m=n=\varphi, \quad c^{\prime}=d=\epsilon \varphi, \quad a^{\prime}=-\varphi_{x}, \quad b=-\varphi_{y},
$$

with $\epsilon= \pm 1$. Then the coefficients of $\Gamma_{1}$ are computed as follows:

$$
\begin{gathered}
m_{1}=\varphi\left(\epsilon \varphi^{2}-(\log \varphi)_{x y}\right), \quad n_{1}=1 / \varphi, \quad a_{1}=a-2(\log \varphi)_{x x}, \\
b_{1}=\varphi a_{x}-\varphi(\log \varphi)_{x x x}-2 \varphi_{x}(\log \varphi)_{x x}-\epsilon \varphi_{y}(\log \varphi)_{x y}+\varphi^{2} \varphi_{y}, \\
c_{1}=0, \quad d_{1}=\varphi\left(\varphi^{2}-\epsilon(\log \varphi)_{x y}\right), \quad a_{1}^{\prime}=\epsilon \varphi_{x} / \varphi^{2}, \\
b_{1}^{\prime}=-\varphi_{y y} / \varphi+2 \varphi_{y}^{2} / \varphi^{2}+\epsilon \varphi_{x x} / \varphi-\epsilon a, \quad c_{1}^{\prime}=\epsilon / \varphi, \quad d_{1}^{\prime}=0 .
\end{gathered}
$$

The conditions given in (7.4) and (7.5) so that $\Gamma_{1}$ is a linear complex are seen to be

$$
a=\varphi_{x x} / \varphi+Y(y), \quad m_{1} / c_{1}^{\prime}=\epsilon \varphi^{2}\left(\epsilon \varphi^{2}-(\log \varphi)_{x y}\right)=: k,
$$

where $Y$ is a function of $y$ and $k$ is a constant. Next we check the integrability condition (8.1). It shows that

$$
Y^{\prime}=0, \quad \varphi\left(a+\epsilon b^{\prime}\right)=\varphi_{x x}+\epsilon \varphi_{y y}, \quad b_{x}^{\prime}=4 \epsilon \varphi \varphi_{y} .
$$

Hence, $Y=\ell$ is a constant and we have

$$
(\log \varphi)_{x y}=\epsilon\left(\varphi^{2}-k \varphi^{-2}\right) .
$$

The coefficients are

$$
\begin{gathered}
m=\varphi, \quad n=\epsilon \varphi, \\
a=\frac{\varphi_{x x}}{\varphi}+\ell, \quad b=-\epsilon \varphi_{y}, \quad c=0, \quad d=\epsilon \varphi, \\
a^{\prime}=-\varphi_{x}, \quad b^{\prime}=\frac{\varphi_{y y}}{\varphi}-\epsilon \ell, \quad c^{\prime}=\varphi, \quad d^{\prime}=0 .
\end{gathered}
$$

Now it is easy to see that a simple change of variables $(x, y)$ shows that we can assume $k= \pm 1$. The result is summarized in the following theorem. 
TheOREM 8.3. (Wilczynski [W11]) The congruences $\Gamma_{0}$ and $\Gamma_{1}$ both belong to linear complexes if and only if there exists a non-vanishing function $\varphi$ satisfying the equation

$$
\frac{\partial^{2} \log \varphi}{\partial x \partial y}=\epsilon\left(\varphi^{2}-k \varphi^{-2}\right),
$$

and the line congruence is given by the system

$$
\begin{aligned}
z_{y} & =\varphi w, \quad z_{x x}=\left(\varphi_{x x} / \varphi+\ell\right) z-\epsilon \varphi_{y} w+\epsilon \varphi w_{y}, \\
w_{x} & =\epsilon \varphi z, \quad w_{y y}=-\varphi_{x} z+\left(\varphi_{y y} / \varphi-\epsilon \ell\right) w+\varphi z_{x},
\end{aligned}
$$

where $\ell$ is any constant, $\epsilon= \pm 1$ and $k= \pm 1$.

We call the function $\varphi$ the potential for the pair $\left\{\Gamma_{0}, \Gamma_{1}\right\}$.

COROLlaRY 8.4. Assume that both $\Gamma_{0}$ and $\Gamma_{1}$ satisfy the condition in the theorem. Let $\Gamma_{2}$ be the (positive) Laplace transform of $\Gamma_{1}$. Then $\Gamma_{2}$ is projectively equivalent to $\Gamma_{0}$. In other words, $\Gamma_{0}$ is doubly periodic.

Proof. By a direct calculation we can see that the potential of the pair $\left\{\Gamma_{1}, \Gamma_{2}\right\}$ is equal to $1 / \varphi$.

COROLlaRY 8.5. The congruence $\Gamma_{0}$ is projectively equivalent to $\Gamma_{1}$ if and only if $\varphi= \pm 1$.

Example 8.6. The focal surfaces when $\varphi= \pm 1$ are hyperboloids.

The lines in Figure 2 are the tangent lines to $x$-curves of the right surface and are tangent to the left surface along a curve parametrized by $x$; and the lines in Figure 3 are the tangent lines to $y$-curves of the left surface and are tangent to the right surface along a curve parametrized by $y$. The figures above were drawn by W. Rossman.

\section{Invariants of focal surfaces}

A line congruence gives a correspondence between two focal surfaces. In this section, we compute such a line congruence when both of the focal surfaces are quadrics. This was first done by Wilczynski and we will follow a part of his description of such congruences. To do this, we need to compute invariants of focal surfaces in terms of invariants of line congruences. We continue to work on the real field. 


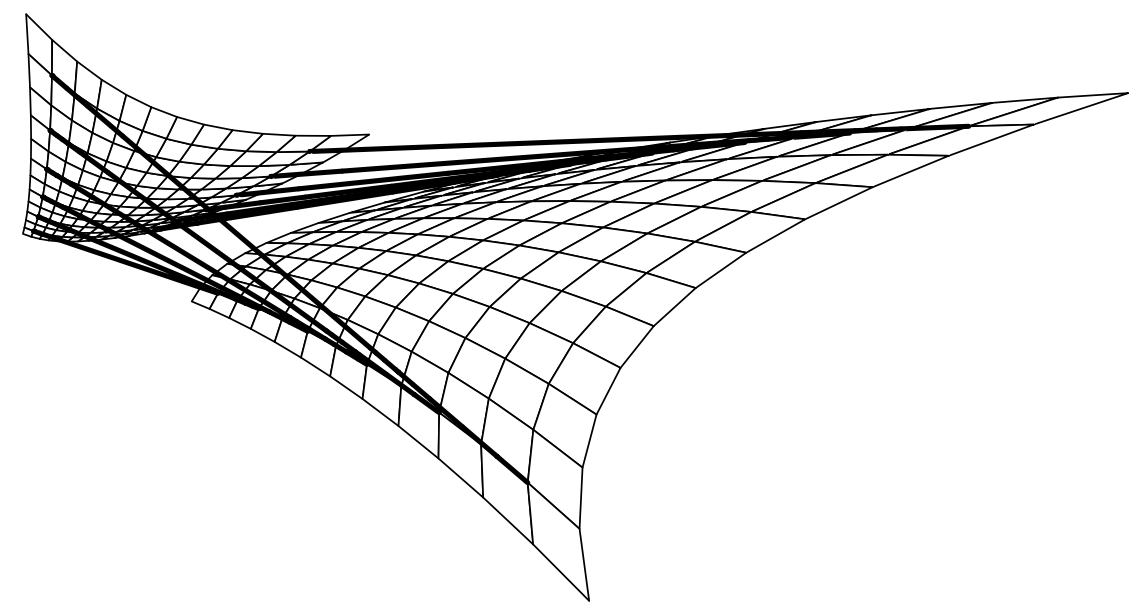

FIGURE 2. Line congruence between two hyperboloids 1 .

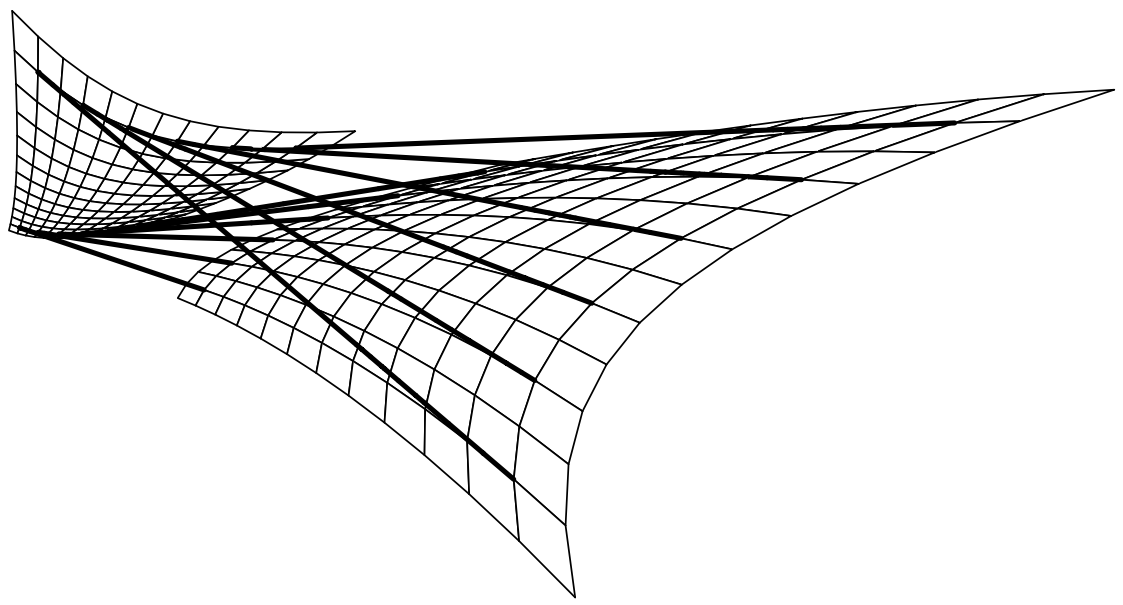

FIGURE 3. Line congruence between two hyperboloids 2. 


\subsection{Invariants of focal surfaces}

Let a projective frame $\left\{e_{1}, e_{2}, e_{3}, e_{4}\right\}$ be a line congruence, as explained in Section 6 . We regard the frame $\left\{e_{1}, e_{2}, e_{3}, e_{4}\right\}$ as a frame associated with the first focal surface $e_{1}$. Then by the process introduced in Section 2, we can compute the invariants. Let $\omega$ denote the associated coframe; see (6.6):

$$
\omega=\left(\begin{array}{cccc}
0 & m d y & d x & 0 \\
n d x & 0 & 0 & d y \\
a d x+m n d y & b d x+m_{x} d y & c d x & d d x \\
a^{\prime} d y+n_{y} d x & b^{\prime} d y+m n d x & c^{\prime} d y & d^{\prime} d y
\end{array}\right)
$$

As it is necessary to normalize the coframe, as required in Proposition 2.1, we consider a change of frame of the form

$$
\left(\begin{array}{l}
\bar{e}_{1} \\
\bar{e}_{2} \\
\bar{e}_{3} \\
\bar{e}_{4}
\end{array}\right)=\left(\begin{array}{cccc}
\lambda & 0 & 0 & 0 \\
g r & g & 0 & 0 \\
h s & 0 & h & 0 \\
0 & v p & v q & v
\end{array}\right)\left(\begin{array}{l}
e_{1} \\
e_{2} \\
e_{3} \\
e_{4}
\end{array}\right)
$$

for which we assume $\lambda g h v>0$. The new coframe $\bar{\omega}$ relative to this frame should satisfy

$$
\bar{\omega}_{1}^{1}=0, \quad \bar{\omega}_{2}^{2}+\bar{\omega}_{3}^{3}=0, \quad \bar{\omega}_{4}^{4}=0 .
$$

As we get

$$
\begin{aligned}
d \bar{e}_{1}= & (d \log \lambda-r m d y-s d x) \bar{e}_{1}+\frac{\lambda m}{g} d y \bar{e}_{2}+\frac{\lambda}{h} d x \bar{e}_{3}, \\
d \bar{e}_{2}= & (d(g r)+g n d x) e_{1}+(d \log g+(r m-p) d y)\left(\bar{e}_{2}-g r e_{1}\right) \\
& +\frac{g}{h}(r d x-q d y)\left(\bar{e}_{3}-h s e_{1}\right)+\frac{g}{v} d y \bar{e}_{4}, \\
d \bar{e}_{3}= & \{d(h s)+h(a d x+m n d y)\} e_{1} \\
& +\frac{h}{g}\left\{(b-d p) d x+\left(m_{x}+s m\right) d y\right\}\left(\bar{e}_{2}-g r e_{1}\right) \\
& +\{d \log h+(c+s-q d) d x\}\left(\bar{e}_{3}-h s e_{1}\right)+\frac{h d}{v} d x \bar{e}_{4}, \\
d \bar{e}_{4} \equiv & \left\{(v q d) d x+\left(v d^{\prime}+v p\right) d y+d v\right\} e_{4} \quad\left(\bmod e_{1}, e_{2}, e_{3}\right),
\end{aligned}
$$


the condition we need is the following:

$$
\begin{gathered}
d \log \lambda-s d x-r m d y=0, \\
d \log v+q d d x+\left(d^{\prime}+p\right) d y=0, \\
d \log (g h)+(c+s-q d) d x+(r m-p) d y=0 .
\end{gathered}
$$

By assuming

$$
g=\lambda m, \quad h=\lambda,
$$

we see that

$$
\bar{\omega}^{1}:=\bar{\omega}_{1}^{2}=d y, \quad \bar{\omega}^{2}:=\bar{\omega}_{1}^{3}=d x .
$$

Then we have

$$
\bar{\omega}_{2}^{4}=\frac{\lambda m}{v} d y, \quad \bar{\omega}_{3}^{4}=\frac{\lambda d}{v} d x
$$

and the fundamental tensor of the new frame is

$$
\bar{h}=\frac{\lambda}{v}\left(\begin{array}{cc}
m & 0 \\
0 & d
\end{array}\right) .
$$

Hence, the condition $|\operatorname{det} \bar{h}|=1$ is satisfied when

$$
\lambda^{2}|m d|=v^{2} .
$$

In particular, the conditions $(9.1 \mathrm{a}-\mathrm{c})$ give the identity

$$
d \log \left(\lambda^{3} v m\right)+c d x+d^{\prime} d y=0 .
$$

On the other hand, we know that we can set

$$
c=-f_{x} / f, \quad d^{\prime}=-f_{y} / f
$$

by (6.10). Then we may assume that $\lambda^{3} m v=f>0$. In the following, we set $\epsilon_{1}=1$ when $\bar{h}$ is positive definite and $\epsilon_{1}=-1$ when it is indefinite. To be more precise, assume $m>0$ and $\epsilon_{1}$ denotes the sign of $d$. Furthermore, let us drop overlines from the notation. Then, from $\epsilon_{1} \lambda^{2} m d=v^{2}$ and $\lambda^{3} m v=f$, we have

$$
\begin{gathered}
\lambda=f^{1 / 4}\left(\epsilon_{1} m^{3} d\right)^{-1 / 8}, \quad g=\lambda m, \quad h=\lambda, \quad v=f^{1 / 4}\left(\epsilon_{1} m d^{3}\right)^{1 / 8}, \\
\omega_{2}^{4}=|m / d|^{1 / 2} d y, \quad \omega_{3}^{4}=\epsilon_{1}|d / m|^{1 / 2} d x .
\end{gathered}
$$

From (9.1a), we have

$$
\begin{gathered}
r=\frac{1}{m}(\log \lambda)_{y}=\frac{1}{8 m}\left(\frac{2 f_{y}}{f}-\frac{d_{y}}{d}-\frac{3 m_{y}}{m}\right), \\
s=(\log \lambda)_{x}=\frac{1}{8}\left(\frac{2 f_{x}}{f}-\frac{d_{x}}{d}-\frac{3 m_{x}}{m}\right),
\end{gathered}
$$


and, from (9.1b),

$$
p=\frac{1}{8}\left(\frac{6 f_{y}}{f}-\frac{3 d_{y}}{d}-\frac{m_{y}}{m}\right), \quad q=-\frac{1}{8 d}\left(\frac{2 f_{x}}{f}+\frac{3 d_{x}}{d}+\frac{m_{x}}{m}\right) .
$$

Thus we have a normalized coframe as follows:

$$
\begin{aligned}
\omega_{2}^{1}= & m(d r-r d \log \lambda+n d x+(p r+q s) d y), \\
\omega_{3}^{1}= & d s+\left(a-r b+r d p-c s-s^{2}+q d s\right) d x+\left(m n-r m_{x}-r s m\right) d y, \\
\omega_{2}^{2}= & d \log \lambda m+(r m-p) d y, \\
\omega_{2}^{3}= & m(r d x-q d y), \\
m \omega_{3}^{2}= & (b-p d) d x+\left(m_{x}+s m\right) d y, \\
\omega_{3}^{3}= & d \log \lambda+(c+s-q d) d x, \\
\lambda m \omega_{4}^{2}= & d(v p)+v(m n+b q) d x+v\left(b^{\prime}+q m_{x}\right) d y, \\
\lambda \omega_{4}^{3}= & d(v q)+v c q d x+v c^{\prime} d y, \\
\lambda \omega_{4}^{1}= & -r\left\{d(v p)+v(b q+m n) d x+v\left(b^{\prime}+q m_{x}\right) d y\right\} \\
& -s\left\{d(v q)+c v q d x+v c^{\prime} d y\right\} \\
& +v\left\{\left(n_{y}+a q+p n\right) d x+\left(a^{\prime}+q m n\right) d y\right\} .
\end{aligned}
$$

By setting

$$
\mu=|m / d|^{1 / 2}
$$

the fundamental tensor is

$$
h_{11}=\mu, \quad h_{12}=h_{21}=0, \quad h_{22}=\epsilon_{1} / \mu ;
$$

namely, the fundamental form $\varphi_{2}$ of the surface $e_{1}$ is given by

$$
\varphi_{2}=\mu d y^{2}+\epsilon_{1} \mu^{-1} d x^{2} .
$$

We next compute the cubic tensor $h_{i j k}$, which is, by definition,

$$
\sum_{k} h_{i j k} \omega^{k}=d h_{i j}-\sum_{k} h_{i k} \omega_{j+1}^{k+1}-\sum_{k} h_{k j} \omega_{i+1}^{k+1} \quad \text { for } 1 \leq i, j, k \leq 2 .
$$

(Note that the indexing of $\omega$ differs by 1 from that used in Section 2.) In fact, we have

$$
\begin{aligned}
h_{111} d y+h_{112} d x & =d h_{11}-2 h_{11} \omega_{2}^{2} \\
& =d \mu-2 \mu(d \log \lambda m+(r m-p) d y)
\end{aligned}
$$


and

$$
\begin{aligned}
h_{221} d y+h_{222} d x & =d h_{22}-2 h_{22} \omega_{3}^{3} \\
& =d\left(\epsilon_{1} / \mu\right)-\left(2 \epsilon_{1} / \mu\right)(d \log \lambda+(c+s-q d) d x) .
\end{aligned}
$$

Hence, computation shows that

$$
h_{111}=\mu \beta, \quad h_{112}=-\mu \gamma, \quad h_{122}=-\epsilon_{1} \mu^{-1} \beta, \quad h_{222}=\epsilon_{1} \mu^{-1} \gamma,
$$

where

$$
\beta=\frac{1}{4}\left(\log \frac{f^{2}}{d^{3} m}\right)_{y}, \quad \gamma=\frac{1}{4}\left(\log f^{2} d m^{3}\right)_{x} .
$$

This means that the cubic form $\varphi_{3}$ of the surface $e_{1}$ is given by

$$
\varphi_{3}=\mu \beta d y^{3}-\mu \gamma d x d y^{2}-\epsilon_{1} \mu^{-1} \beta d x^{2} d y+\epsilon_{1} \mu^{-1} \gamma d x^{3} .
$$

For the second focal surface $e_{2}$, the fundamental form $\psi_{2}$ and the cubic form $\psi_{3}$ of the surface $e_{2}$ are given by

$$
\begin{gathered}
\psi_{2}=\mu_{1} d x^{2}+\epsilon_{2} \mu_{1}^{-1} d y^{2}, \\
\psi_{3}=\mu_{1} \beta_{1} d x^{3}-\mu_{1} \gamma_{1} d y d x^{2}-\epsilon_{2} \mu_{1}^{-1} \beta_{1} d y^{2} d x+\epsilon_{2} \mu_{1}^{-1} \gamma_{1} d y^{3},
\end{gathered}
$$

where

$$
\begin{gathered}
\epsilon_{2}=\operatorname{sign}\left(n c^{\prime}\right), \quad \mu_{1}=\left|n / c^{\prime}\right|^{1 / 2}, \\
\beta_{1}=\frac{1}{4}\left(\log \frac{f^{2}}{n c^{\prime 3}}\right)_{x}, \quad \gamma_{1}=\frac{1}{4}\left(\log f^{2} n^{3} c^{\prime}\right)_{y} .
\end{gathered}
$$

\subsection{Line congruences both of whose focal surfaces are quadrics}

We next describe line congruences whose focal surfaces are quadrics. As the signatures of $\varphi_{2}$ and $\psi_{2}$ are important in the following argument, we assume that $m>0$ and $n>0$, which is possible by Lemma 6.6 and set $\epsilon_{1}=\operatorname{sign}(d), \epsilon_{2}=\operatorname{sign}\left(c^{\prime}\right)$ and $\epsilon=\epsilon_{1} \epsilon_{2}$. If $\epsilon=1$, both of $\varphi_{2}$ and $\psi_{2}$ have the same signature and, if $\epsilon=-1$, the signature of $\varphi_{2}$ is opposite to the signature of $\psi_{2}$. By Theorem 2.3, and by referring to (9.5) and (9.9), both focal surfaces are quadrics if and only if

$$
\begin{aligned}
& \left(\log \frac{f^{2}}{d^{3} m}\right)_{y}=0, \quad\left(\log f^{2} d m^{3}\right)_{x}=0, \\
& \left(\log \frac{f^{2}}{n c^{\prime 3}}\right)_{x}=0, \quad\left(\log f^{2} n^{3} c^{\prime}\right)_{y}=0 .
\end{aligned}
$$


From these relations, we see that

$$
(\log m d)_{x y}=\left(\log n c^{\prime}\right)_{x y}=0
$$

and

$$
\left(\log m n^{3} c^{\prime} d^{3}\right)_{y}=0, \quad\left(\log m^{3} n c^{\prime 3} d\right)_{x}=0 .
$$

Then, for some positive-valued scalar functions $X_{1}(x), X_{2}(x), X_{3}(x), Y_{1}(y), Y_{2}(y)$, $Y_{3}(y)$, we may set

$$
m d=\epsilon_{1} X_{1}^{2} Y_{1}^{2}, \quad n c^{\prime}=\epsilon_{2} X_{2}^{2} Y_{2}^{2},
$$

and

$$
m n^{3} c^{\prime} d^{3}=\epsilon X_{3}(x), \quad m^{3} n c^{\prime 3} d=\epsilon Y_{3}(y) .
$$

Hence, we have

$$
X_{3}=k^{2}\left(X_{1} X_{2}\right)^{8}, \quad Y_{3}=\frac{1}{k^{2}}\left(Y_{1} Y_{2}\right)^{8},
$$

for some positive constant $k$. We next define $\varphi$ by

$$
m=Y_{1}^{3} Y_{2} \varphi .
$$

Then we have

$$
n=k X_{1} X_{2}^{3} \varphi, \quad d=\frac{\epsilon_{1} X_{1}^{2}}{Y_{1} Y_{2} \varphi}, \quad c^{\prime}=\frac{\epsilon_{2} Y_{2}^{2}}{k X_{1} X_{2} \varphi} .
$$

Now, in view of Lemma 6.6, by a change of variables from $(x, y ; z, w)$ to $(\bar{x}=$ $X(x), \bar{y}=Y(y) ; \bar{z}=z / \lambda(x), \bar{w}=w / \mu(y))$, the ratios $m / n$ and $d / c^{\prime}$ become $\mu^{2} X_{x} Y_{1}^{3} Y_{2} /\left(k \lambda^{2} Y_{y} X_{1} X_{2}^{3}\right)$ and $\epsilon k \mu^{2} Y_{y}^{3} X_{1}^{3} X_{2} /\left(\lambda^{2} X_{x}^{3} Y_{1} Y_{2}^{3}\right)$, respectively. This shows that we may assume that $m=n$ and $c^{\prime}=\epsilon d$ by solving the equations $X_{x} / \lambda^{2}=$ $k X_{1} X_{2}^{3}, \lambda^{2} X_{x}^{3}=k X_{1}^{3} X_{2}, Y_{y} / \mu^{2}=Y_{1}^{3} Y_{2}$ and $\mu^{2} Y_{y}^{3}=Y_{1} Y_{x}^{3}$. Then we can suppose that $X_{1}, X_{2}, Y_{1}$ and $Y_{2}$ are constants. Namely, we have reduced to the case $m=n=\varphi$ and $\epsilon_{2} c^{\prime}=\epsilon_{1} d=k_{1} / \varphi$ for some constant $k_{1}$. Applying Lemma 6.6 once more, we see that we may assume $k_{1}=1$ :

$$
m=n=\varphi, \quad d=\epsilon_{1} / \varphi, \quad c^{\prime}=\epsilon_{2} / \varphi .
$$

In this case, (9.10) shows that $f \varphi$ is constant, and from (9.2) we see that

$$
c=\varphi_{x} / \varphi, \quad d^{\prime}=\varphi_{y} / \varphi .
$$

Then a check of the integrability (6.9) implies that

$$
\begin{aligned}
& a^{\prime}=0, \quad b=0, \quad a_{y}=2 \varphi \varphi_{x}, \quad b_{x}^{\prime}=2 \varphi \varphi_{y}, \\
& (\log \varphi)_{x x}=a+\frac{\epsilon_{1} b^{\prime}}{\varphi^{2}}, \quad(\log \varphi)_{y y}=b^{\prime}+\frac{\epsilon_{2} a}{\varphi^{2}}
\end{aligned}
$$


and

$$
(\log \varphi)_{x y}=\varphi^{2}-\frac{\epsilon}{\varphi^{2}}
$$

Then we have the following theorem due to Wilczynski.

THEOREM 9.1. Assume that both focal surfaces are quadrics. Then there exists a non-vanishing function $\varphi$ such that

$$
m=n=\varphi, \quad d=\frac{\epsilon_{1}}{\varphi}, \quad c^{\prime}=\frac{\epsilon_{2}}{\varphi} .
$$

Unless $\varphi^{2}=1$ and $\epsilon=1$, it must satisfy the following system of equations:

$$
\begin{gathered}
(\log \varphi)_{x y}=\varphi^{2}-\epsilon \varphi^{-2}, \\
2 \varphi \varphi_{x}=\left(\left(\varphi^{2}(\log \varphi)_{x x}-\epsilon_{1}(\log \varphi)_{y y}\right) /\left(\varphi^{2}-\epsilon \varphi^{-2}\right)\right)_{y}, \\
2 \varphi \varphi_{y}=\left(\left(\varphi^{2}(\log \varphi)_{y y}-\epsilon_{2}(\log \varphi)_{x x}\right) /\left(\varphi^{2}-\epsilon \varphi^{-2}\right)\right)_{x} .
\end{gathered}
$$

The coefficients of the congruence are given by

$$
\begin{gathered}
a=\left(\varphi^{2}(\log \varphi)_{x x}-\epsilon_{1}(\log \varphi)_{y y}\right) /\left(\varphi^{2}-\epsilon \varphi^{-2}\right), \\
b^{\prime}=\left(\varphi^{2}(\log \varphi)_{y y}-\epsilon_{2}(\log \varphi)_{x x}\right) /\left(\varphi^{2}-\epsilon \varphi^{-2}\right), \\
b=0, \quad c=\varphi_{x} / \varphi, \quad a^{\prime}=0, \quad d^{\prime}=\varphi_{y} / \varphi .
\end{gathered}
$$

Remark 9.2. For the line congruence above to be a $W$-congruence, the condition that $\epsilon=1$ and $\varphi^{2}=1$ is necessary. When $\varphi=1$ and $\epsilon=1, a$ and $b^{\prime}$ are seen to be constants and must satisfy $a+b^{\prime}=0$. The system is then written as

$$
z_{y}=w, \quad w_{x}=z, \quad z_{x x}=a z+w_{y}, \quad w_{y y}=-a w+z_{x}
$$

where $a$ is a constant and this case is thus included in Theorem 8.3. We refer to Section 10.2. Getting solutions of the above system in Theorem 9.1 is an open problem.

\section{Construction of $W$-congruences}

Fubini and Čeck [FC1] gave a method for constructing $W$-congruences with a given surface as one of the focal surfaces. As this method is fundamental for discussing $W$-congruences, we give a summary of the related computations. One application is the construction of all $W$-congruences whose focal surfaces are quadrics. Section 10.3 treats the composition formula of two $W$-congruences, starting from a given surface. 
10.1. A description of $W$-congruences

Let the surface $z$ be given by the system

$$
z_{x x}=\theta_{x} z_{x}+b z_{y}+p z, \quad z_{y y}=c z_{x}+\theta_{y} z_{y}+q z .
$$

We consider a family of curves on the surface infinitesimally written as $A d y-$ $B d x=0$, where $A$ and $B$ are non-vanishing functions on the surface. Let

$$
w=\mu z+2\left(A z_{x}+B z_{y}\right)
$$

be any point on the tangent line of curves of the given family, where $\mu$ is a scalar parameter on the line. We are going to determine $\mu$ so that the point $w$ is the focal point. We first check the condition that the point $z$ lies on the tangent plane at $w$ of the second surface, i.e. the condition $z \wedge w \wedge w_{x} \wedge w_{y}=0$. As

$$
\begin{aligned}
& w_{x}=\left(\mu+2 A_{x}+2 A \theta_{x}\right) z_{x}+2\left(B_{x}+b A\right) z_{y}+2 B z_{x y}+\left(\mu_{x}+2 p A\right) z, \\
& w_{y}=2\left(A_{y}+c B\right) z_{x}+\left(\mu+2 B_{y}+2 B \theta_{y}\right) z_{y}+2 A z_{x y}+\left(\mu_{y}+2 q B\right) z,
\end{aligned}
$$

the condition is seen to be the identity

$$
\mu=-A_{x}-B_{y}-A \theta_{x}-B \theta_{y}+\frac{B\left(A_{y}+c B\right)}{A}+\frac{A\left(B_{x}+b A\right)}{B} .
$$

Furthermore, a computation shows that

$$
\begin{aligned}
& w_{x}=\frac{1}{B}\left(B_{x}+b A\right) w+E z-\lambda z_{x}+2 B z_{x y}, \\
& w_{y}=\frac{1}{A}\left(A_{x}+c B\right) w+F z+\lambda z_{y}+2 A z_{x y},
\end{aligned}
$$

where

$$
\begin{gathered}
\lambda=-A_{x}+B_{y}-\theta_{x} A+\theta_{y} B+\frac{A}{B}\left(B_{x}+b A\right)-\frac{B}{A}\left(A_{y}+c B\right), \\
E=\mu_{x}+2 p A-\mu \frac{B_{x}+b A}{B}, \\
F=\mu_{y}+2 q B-\mu \frac{A_{y}+c B}{A} .
\end{gathered}
$$

In particular,

$$
\begin{aligned}
A w_{x}-B w_{y}= & \left(A \mu_{x}-B \mu_{y}+2 A^{2} p-2 B^{2} q\right) z \\
& +\left(A \mu+2 A A_{x}-2 B A_{y}-2 c B^{2}+2 A^{2} \theta_{x}\right) z_{x} \\
& -\left(B \mu+2 B B_{y}-2 A B_{x}-2 b A^{2}+2 B^{2} \theta_{y}\right) z_{y} .
\end{aligned}
$$


Hence, the identity (10.2) implies that the vector $A w_{x}-B w_{y}$ is pointing in the direction of the lines of the congruence. In fact, if we set

$$
N=\lambda \mu+2 A E-2 B F,
$$

then we get a relation which is the reverse of (10.1):

$$
N z=-v w+2\left(A w_{x}-B w_{y}\right)
$$

where

$$
v=-B_{y}-\theta_{y} B+A_{x}+\theta_{x} A+\frac{A}{B}\left(B_{x}+b A\right)-\frac{B}{A}\left(A_{y}+c B\right) .
$$

We assume that $N \neq 0$ in the following so that $w$ is non-degenerate.

PROPOSITION 10.1. The line congruence joining $z$ and $w$ is a $W$-congruence if and only if

$$
\left(\frac{A_{y}+c B}{A}\right)_{x}=\left(\frac{B_{x}+b A}{B}\right)_{y}
$$

Proof. If the congruence is a $W$-congruence, then the $x$-curves are asymptotic curves of the surface $w$, namely it holds that $w \wedge w_{x} \wedge w_{y} \wedge w_{x x}=0$. By a computation, this is seen to be equivalent to (10.4). The symmetry of (10.4) implies that the $y$-curves are also asymptotic.

We remark that the conformal structure of $w$ is given by the 2 -form

$$
N\left(4 \alpha(B d x+A d y)^{2}-2 N d x d y\right),
$$

where

$$
\alpha=\left(\frac{A_{y}+c B}{A}\right)_{x}-\left(\frac{B_{x}+b A}{B}\right)_{y} .
$$

We next compute the system defining the surface $w$, assuming that the congruence is a $W$-congruence. The condition (10.4) shows the existence of a function $\varphi$ such that

$$
\frac{A_{y}+c B}{A}=\frac{\varphi_{y}}{\varphi}, \quad \frac{B_{x}+b A}{B}=\frac{\varphi_{x}}{\varphi} .
$$

Replacing $A / \varphi$ and $B / \varphi$ with $A$ and $B$, the condition (10.4) can be reduced to

$$
A_{y}+c B=0, \quad B_{x}+b A=0 .
$$

In this case

$$
\begin{aligned}
& \mu=-A_{x}-B_{y}-\theta_{x} A-\theta_{y} B \\
& \lambda=-A_{x}+B_{y}-\theta_{x} A+\theta_{y} B
\end{aligned}
$$


and

$$
N z=\lambda w+2 A w_{x}-2 B w_{y}
$$

From the identities

$$
\begin{aligned}
& w_{x}=\left(\mu_{x}+2 A p\right) z-\lambda z_{x}+2 B z_{x y}, \\
& w_{y}=\left(\mu_{y}+2 B q\right) z+\lambda z_{y}+2 A z_{x y},
\end{aligned}
$$

we know that the system of differential equations for the surface $w$ is

$$
\begin{aligned}
& w_{x x}=\bar{\theta}_{x} w_{x}+\bar{b} w_{y}+\bar{p} w, \\
& w_{y y}=\bar{c} w_{x}+\bar{\theta}_{y} w_{y}+\bar{q} w,
\end{aligned}
$$

where

$$
\begin{gathered}
\bar{\theta}_{x}=\theta_{x}+(\log N)_{x}, \quad \bar{\theta}_{y}=\theta_{y}+(\log N)_{y}, \\
\bar{b}=-b-\frac{B}{A}(\log N)_{x}, \quad \bar{c}=-c-\frac{A}{B}(\log N)_{y}, \\
\bar{p}=p+b_{y}+b \theta_{y}+\frac{\lambda}{2 A}(\log N)_{x}, \\
\bar{q}=q+c_{x}+c \theta_{x}-\frac{\lambda}{2 B}(\log N)_{y} .
\end{gathered}
$$

Remark 10.2. By using Lemma 10.4 proven later, we have a remarkable identity:

$$
\bar{b} \bar{c}=b c-(\log N)_{x y} .
$$

For later use, we set

$$
\mathcal{L}=A A_{x x}-\frac{1}{2} A_{x}^{2}+A^{2} L, \quad \mathcal{M}=B B_{y y}-\frac{1}{2} B_{y}^{2}+B^{2} M,
$$

then

$$
N=2(\mathcal{M}-\mathcal{L})
$$

Let us make a remark on the case when $N$ is a constant. In this case, the system satisfied by $w$ is nothing but the dual of the system satisfied by $z$. Hence, by recalling the reasoning in Section 7.1, we see that the line congruence $\{z, w\}$ belongs to a linear complex.

10.2. W-congruences whose focal surfaces are quadrics

Let us consider the special case where the surface $z$ is a quadric and the congruence is a $W$-congruence. As $b=c=0$ (and, hence, we may assume that $p=q=0$ and $\theta$ is 
constant), the condition (10.4) implies that $A=X$ is a function of $x$ and $B=Y$ is a function of $y$. Then we have

$$
\begin{aligned}
& \mu=-X^{\prime}-Y^{\prime}, \quad \lambda=-X^{\prime}+Y^{\prime}, \\
& N=X^{\prime 2}-Y^{\prime 2}-2 X X^{\prime \prime}+2 Y Y^{\prime \prime}, \\
& \bar{b}=2 Y X^{\prime \prime \prime} / N, \quad \bar{c}=2 X Y^{\prime \prime \prime} / N .
\end{aligned}
$$

Here $\left\{^{\prime}\right\}$ means derivation relative to the respective variable. For $\bar{b}=\bar{c}=0$, it is necessary and sufficient that $X^{\prime \prime \prime}(x)=Y^{\prime \prime \prime}(y)=0$ because $A$ and $B$ are assumed to not be zero.

THEOREM 10.3. When $X$ and $Y$ are polynomials of degree at most two, the surface $w$ is also a quadric.

Assume that $X$ and $Y$ are polynomials of degree at most two; in particular, $N$ is constant. Then the system for $w$ is

$$
w_{x x}=\theta_{x} w_{x}+p w, \quad w_{y y}=\theta_{y} w_{y}+q w,
$$

which means that the surface $w$ satisfies the same system as for $z$; geometrically, $w$ is a projective transformation of $z$. An explicit correspondence is given below.

Assume, for simplicity, that $p=q=0$ and $\theta$ is constant; the surface $z$ is parametrized as $z=[1, x, y, x y]$ in homogeneous coordinates. Set

$$
X=p_{1} x^{2}+2 p_{2} x+p_{3} \quad \text { and } \quad Y=q_{1} y^{2}+2 q_{2} y+q_{3}
$$

Then the surface $w$ is given by

$$
\begin{aligned}
w=\left[-p_{2}-q_{2}-p_{1} x-q_{1} y, p_{3}+\left(p_{2}-q_{2}\right) x-q_{1} x y,\right. & \\
& \left.q_{3}+\left(q_{2}-p_{2}\right) y-p_{1} x y, q_{3} x+p_{3} y+\left(p_{2}+q_{2}\right) x y\right] .
\end{aligned}
$$

Namely,

$$
w=g z, \quad g=\left(\begin{array}{cccc}
-p_{2}-q_{2} & -p_{1} & -q_{1} & 0 \\
p_{3} & p_{2}-q_{2} & 0 & -q_{1} \\
q_{3} & 0 & q_{2}-p_{2} & -p_{1} \\
0 & q_{3} & p_{3} & p_{2}+q_{2}
\end{array}\right),
$$

where det $g=p_{2}^{2}-q_{2}^{2}-p_{1} p_{3}+q_{1} q_{3}$. To simplify the representation of the congruence, we introduce a new parametrization of the surface by defining new coordinates $(\xi, \eta)$ by

$$
\partial_{\xi}=X \partial_{x}+Y \partial_{y}, \quad \partial_{\eta}=\left(X \partial_{x}-Y \partial_{y}\right) / \operatorname{det}(g) .
$$


We set

$$
\bar{z}=\rho z, \quad \bar{w}=\rho w, \quad \text { where } \rho=(X Y)^{-1 / 2} .
$$

Then it is easy to see that the congruence is written as

$$
\bar{w}=\partial_{\xi} \bar{z}, \quad \bar{z}=\partial_{\eta} \bar{w} .
$$

Thus, the parametrization by $(\xi, \eta)$ defines a net on the surface associated to the congruence. In this way, we get all $W$-congruences whose focal surfaces are quadrics.

We add a few remarks. When one and only one of $X$ and $Y$ is a polynomial of degree at most two, then the surface $w$ is ruled. Thus, we get $W$-congruences joining a quadratic surface and a ruled surface.

The second remark is on the case where $w$ is not ruled: $\overline{b c} \neq 0$. Then

$$
(\log \bar{b})_{x y}=-(\log N)_{x y}=N_{x} N_{y} / N=\overline{b c} .
$$

Similarly, $(\log \bar{c})_{x y}=\overline{b c}$. Hence, by Lemma 2.12, both parameter curves on $w$ belong to respective linear complexes. Conversely, given a surface both of whose parameter curves belong to linear complexes, namely when the condition (2.32) holds, we can construct a $W$-congruence joining the surface and a quadratic surface. We refer to [FC2, Section 47] for details.

\subsection{Composition of $W$-congruences}

Given two $W$-congruences $w_{1}$ and $w_{2}$, each given by

$$
w_{i}=\mu_{i} z+2\left(A_{i} z_{x}+B_{i} z_{y}\right), \quad i=1,2,
$$

Fubini constructed a third surface $z$ that is joined with $w_{1}$ and $w_{2}$ by respective $W$-congruences. Here, we cite his construction.

Let $\lambda_{i}, \mu_{i}$ and $N_{i}$ be the corresponding quantities and define

$$
\begin{aligned}
A & =c^{1} A_{1}+c^{2} A_{2}, \quad B=c^{1} B_{1}+c^{2} B_{2}, \\
\lambda & =c^{1} \lambda_{1}+c^{2} \lambda_{2}, \quad \mu=c^{1} \mu_{1}+c^{2} \mu_{2},
\end{aligned}
$$

where $c^{1}$ and $c^{2}$ are constants, and define $N$ by the formula (10.3). As $N$ is quadratic in $A$ and $B$, it is possible to set

$$
N=\left(c^{1}\right)^{2} N_{1}+2 c^{1} c^{2} N_{12}+\left(c^{2}\right)^{2} N_{2},
$$

by appropriately defining $N_{12}$. In fact, we have

$N_{12}=\frac{1}{2}\left(\lambda_{1} \mu_{2}+\lambda_{2} \mu_{1}\right)+4\left(A_{1} A_{2} p-B_{1} B_{2} q\right)+\left(A_{1} \mu_{2 x}+A_{2} \mu_{1 x}-B_{1} \mu_{2 y}-B_{2} \mu_{1 y}\right)$. 
Furthermore, a computation shows that

$$
\begin{aligned}
& \frac{N_{x}}{A}=\mu_{x x}-\theta_{x} \mu_{x}+b \mu_{y}-\mu\left(b_{y}+b \theta_{y}\right)+4 B b q+4 A_{x} p+2 A p_{x}+2 B p_{y}, \\
& -\frac{N_{y}}{A}=\mu_{y y}-\theta_{v} \mu_{v}+c \mu_{x}-\mu\left(c_{x}+c \theta_{x}\right)+4 A c p+4 B_{y} q+2 B q_{y}+2 A q_{x},
\end{aligned}
$$

and note that the right-hand sides are linear in $A, B$ and $\mu$. Hence, we have

$$
\frac{N_{x}}{2 A}=c^{1} \frac{N_{1 x}}{2 A_{1}}+c^{2} \frac{N_{2 x}}{2 A_{2}} .
$$

From this follows

$$
\left(N_{12}\right)_{x}=\frac{A_{2}}{2 A_{1}} N_{1 x}+\frac{A_{1}}{2 A_{2}} N_{2 x} .
$$

Similarly,

$$
\left(N_{12}\right)_{y}=\frac{B_{2}}{2 B_{1}} N_{1 y}+\frac{B_{1}}{2 B_{2}} N_{2 y} .
$$

LEMMA 10.4. N satisfies the equation

$$
N_{x y}+\frac{c B}{A} N_{x}+\frac{b A}{B} N_{y}=0 .
$$

The proof is given in Section 13.1 (see Lemma 13.1). We now look for a scalar function $f$ and $g$ so that

$$
f_{x}=\frac{A_{2}}{A_{1}} N_{1 x}, \quad f_{y}=\frac{B_{2}}{B_{1}} N_{1 y} ; \quad g_{x}=\frac{A_{1}}{A_{2}} N_{2 x}, \quad g_{y}=\frac{B_{1}}{B_{2}} N_{2 y} .
$$

From (10.5) and (10.13),

$$
\begin{aligned}
\left(f_{x}\right)_{y} & =\frac{A_{2 y}}{A_{1}} N_{1 x}-\frac{A_{2} A_{1 y}}{A_{1}^{2}} N_{1 x}+\frac{A_{2}}{A_{1}} N_{1 x y} \\
& =-\frac{c B_{2}}{A_{1}} N_{1 x}-\frac{b A_{2}}{B_{1}} N_{1 y}
\end{aligned}
$$

and the same holds for $\left(f_{y}\right)_{x}$. Hence, it is possible to find $f$ by integration. Similarly, we can find $g$. By definition, $f+g-N_{12}$ is constant, and we may assume it is equal to zero. Now we define two new surfaces

$$
\begin{aligned}
& Z_{12}=f z-\lambda_{2} w_{1}+2\left(-A_{2} w_{1 x}+B_{2} w_{1 y}\right), \\
& Z_{21}=g z-\lambda_{1} w_{2}+2\left(-A_{1} w_{2 x}+B_{1} w_{2 y}\right) .
\end{aligned}
$$


Inserting the derivations of $w_{1}$ and $w_{2}$ given in (10.7), we get

$$
\begin{aligned}
Z_{12}= & \left(f-\lambda_{2} \mu_{1}-2 A_{2} \mu_{1 x}-4 A_{1} A_{2} p+2 B_{2} \mu_{1 y}+4 B_{1} B_{2} q\right) z \\
& +2\left(\lambda_{1} A_{2}-\lambda_{2} A_{1}\right) z_{x}+2\left(\lambda_{1} B_{2}-\lambda_{2} B_{1}\right) z_{y}+4\left(B_{2} A_{1}-A_{2} B_{1}\right) z_{x y}, \\
Z_{21}= & \left(g-\lambda_{1} \mu_{2}-2 A_{1} \mu_{2 x}-4 A_{1} A_{2} p+2 B_{1} \mu_{2 y}+4 B_{1} B_{2} q\right) z \\
& +2\left(\lambda_{2} A_{1}-\lambda_{1} A_{2}\right) z_{x}+2\left(\lambda_{2} B_{1}-\lambda_{1} B_{2}\right) z_{y}+4\left(B_{1} A_{2}-A_{1} B_{2}\right) z_{x y} .
\end{aligned}
$$

Therefore,

$$
Z_{12}+Z_{21}=\left(f+g-2 N_{12}\right) z=0 .
$$

Now we write $Z$ for $Z_{12}$ and we show that $Z$ is one of the required surfaces. In fact, by setting

$$
\bar{\mu}=-\lambda_{2}+\frac{\lambda_{1} a}{N_{1}}, \quad \bar{A}=-A_{2}+\frac{A_{1} a}{N_{1}}, \quad \bar{B}=B_{2}-\frac{B_{1} a}{N_{1}},
$$

where $a$ is a constant of integration relative to $f$, we have

$$
Z=\bar{\mu} w_{1}+2\left(\bar{A} w_{1 x}+\bar{B} w_{1 y}\right)
$$

see (10.6). If we denote by $\bar{\theta}, b_{1}$ and $c_{1}$ the invariants $\theta, b$ and $c$ for $w_{1}$, then we see by a simple calculation that

$$
\bar{A}_{y}=-c_{1} \bar{B}, \quad \bar{B}_{x}=-b_{1} \bar{A}
$$

and

$$
\bar{A}_{x}+\bar{\theta}_{x} \bar{A}+\bar{B}_{y}+\bar{\theta}_{y} \bar{B}=\lambda_{2}-\lambda_{1} a / N_{1} .
$$

Hence, we have proved the following theorem.

THEOREM 10.5. Let $\left\{w_{1}, z\right\}$ and $\left\{w_{2}, z\right\}$ be two $W$-congruences. Define a third surface $Z$ by (10.14). Then, both $\left\{Z, w_{1}\right\}$ and $\left\{Z, w_{2}\right\}$ are $W$-congruences. The constants $c^{1}$ and $c^{2}$ are arbitrary and the choice of $f$ and $g$ includes a constant of integration.

\section{Lie quadrics and Demoulin transforms}

In Section 2.4 we have defined the Demoulin frame associated with a surface and the Demoulin transform of the surface. In this section we revisit these notions, following the development by Finikoff and Mayer. We first recall how to attach a quadric called the Lie quadric to each point of the surface, and then prove that the envelope of Lie quadrics generally consists of four surfaces, each being a Demoulin transform. 
We next define Demoulin congruences joining Demoulin transforms and prove that the Demoulin congruence is a $W$-congruence if and only if the original surface is projectively minimal. In the last section, we compute explicitly the invariants of the Demoulin transform.

\subsection{Osculating quadrics}

Let $z=z_{1}(x)+y z_{2}(x)$ be a ruled surface given by the normalized system (1.6). We now define an osculating quadric to this ruled surface.

Given a set of skew three lines on the ruled surface, we generally have a quadric including these lines. If these lines tend to one limit line, then the quadric tends to a limit position, again a quadric. This is called an osculating quadric, which might be degenerate. Assume that the limit quadric $Q$ is defined by the equation ${ }^{\mathrm{t}} z A z=0$. We denote ${ }^{\mathrm{t}} z A z=\langle z, z\rangle$ for simplicity. The limit line is in $Q$ if and only if

$$
\left\langle z_{1}, z_{1}\right\rangle=\left\langle z_{1}, z_{2}\right\rangle=\left\langle z_{2}, z_{2}\right\rangle=0 .
$$

The limit process implies that the first derivatives of the limiting lines are also in $Q$ :

$$
\left\langle z_{1}, z_{1}^{\prime}\right\rangle=\left\langle z_{1}, z_{2}^{\prime}\right\rangle+\left\langle z_{1}^{\prime}, z_{2}\right\rangle=\left\langle z_{2}, z_{2}^{\prime}\right\rangle=0
$$

Differentiating a second time implies

$$
\left\langle z_{1}^{\prime}, z_{1}^{\prime}\right\rangle=\left\langle z_{1}^{\prime}, z_{2}^{\prime}\right\rangle=\left\langle z_{2}^{\prime}, z_{2}^{\prime}\right\rangle=0
$$

in view of (1.6). If we write a general point $z$ in $\mathbb{P}^{3}$ as $w=p^{0} z_{1}+p^{1} z_{2}+p^{2} z_{1}^{\prime}+p^{3} z_{2}^{\prime}$, then

$$
\langle w, w\rangle=2\left(p^{0} p^{3}-p^{1} p^{2}\right)\left\langle z_{1}, z_{2}^{\prime}\right\rangle .
$$

Hence the osculating quadric is defined by the equation $p^{0} p^{3}-p^{1} p^{2}=0$. A part of the condition $\left\langle z_{1}, z_{1}\right\rangle=\left\langle z_{1}, z_{1}^{\prime}\right\rangle=\left\langle z_{1}^{\prime}, z_{1}^{\prime}\right\rangle=0$ implies that the asymptotic tangent belongs to the osculating quadric. As this property does not depend on $y$, all asymptotic lines through the limit ruling line give a ruling of the osculating quadric.

\subsection{Lie quadrics}

Let $z(x, y)$ be a non-degenerate surface:

$$
\begin{aligned}
& z_{x x}=\theta_{x} z_{x}+b z_{y}+p z, \\
& z_{y y}=c z_{x}+\theta_{y} z_{y}+q z .
\end{aligned}
$$


We consider one of the associated ruled surfaces

$$
w(x, s)=z_{y}\left(x, y_{0}\right)+s z\left(x, y_{0}\right),
$$

which consists of tangent lines to $y$-curves parametrized by $x$. The osculating quadric to this ruled surface is determined by the asymptotic directions, as was remarked in the previous section.

Let $s=s(x)$ be such an asymptotic curve. As

$$
\begin{gathered}
w_{x}=z_{x y}+s^{\prime} z+s z_{x}, \\
w_{x x}=z_{x x y}+s^{\prime \prime} z+2 s^{\prime} z_{x}+s z_{x x}, \\
z_{x x y}=\theta_{x} z_{x y}+\left(b c+\theta_{x x}\right) z_{x}+\left(b \theta_{y}+b_{y}+p\right) z_{y}+\left(b q+p_{y}\right) z,
\end{gathered}
$$

and then

$$
w_{x x} \equiv\left(b c+2 s^{\prime}+\theta_{x y}\right) z_{x} \quad\left(\bmod z, z_{y}, w_{x}\right)
$$

in order that $w_{x x} \equiv 0\left(\bmod z, z_{y}, w_{x}\right)$, it is necessary that

$$
b c+2 s^{\prime}+\theta_{x y}=0 .
$$

Hence, we have

$$
w_{x}=z_{x y}+s z_{x}-(1 / 2)\left(b c+\theta_{x y}\right) z .
$$

The asymptotic direction is written as

$$
w_{x}+t w=z_{x y}+s z_{x}+t z_{y}+\left(s t-(1 / 2)\left(b c+\theta_{x y}\right)\right) z,
$$

where $t$ is a line parameter. If we write any point in $\mathbb{P}^{3}$ as $p^{0} z+p^{1} z_{x}+p^{2} z_{y}+p^{3} z_{x y}$, then the quadric consisting of the asymptotic directions above is

$$
p^{0} p^{3}-p^{1} p^{2}=-(1 / 2)\left(b c+\theta_{x y}\right)\left(p^{3}\right)^{2} .
$$

(If the original surface is ruled, then this coincides with the osculating quadric. In fact, $b c=0$ for a ruled surface and $\theta$ is constant for a ruled surface written in the form (1.6).) This quadric is called a Lie quadric, which is the same as the Lie quadratic hypersurface given in Section 2.2 in view of the frame (2.14).

\subsection{Demoulin transforms}

To each point of the surface is associated a Lie quadric. The envelope of Lie quadrics generally consists of four surfaces. We shall see that these four surfaces are nothing but the Demoulin transforms of the original surface, defined in Section 2.4. 
We assume that $b c \neq 0$ so that the surface is not ruled. Any Lie quadric is of the form

$$
w=z_{x y}+s z_{x}+t z_{y}+(s t-\alpha) z, \quad \alpha=\frac{1}{2}\left(b c+\theta_{x y}\right) .
$$

In order that $w$ belongs to the envelope surface, its tangent vector, say $w_{x}$, belongs to the tangent plane of the Lie quadric. By a direct computation, we have

$$
\begin{aligned}
w_{x}= & \left(t+\theta_{x}\right) w+\left(\alpha+s_{x}\right)\left(z_{x}+t z\right) \\
& +\left(b \theta_{y}+b_{y}+p+t_{x}+b s-t^{2}-t \theta_{x}\right)\left(z_{y}+s z\right)+B z,
\end{aligned}
$$

where

$$
B=\left(b q+p_{y}-\alpha_{x}+\alpha \theta_{x}\right)-\left(b_{y}+b \theta_{y}\right) s-b s^{2} .
$$

As the tangent plane of the Lie quadric is spanned by the vectors $w, w_{s}=z_{x}+t z$ and $w_{t}=z_{y}+s z$, the required condition for the vector $w_{x}$ is $B=0$; namely,

$$
b s^{2}+\left(b_{y}+b \theta_{y}\right) s-\left(b q+p_{y}-\alpha_{x}+\alpha \theta_{x}\right)=0 .
$$

Similarly, for $w_{y}$ we have

$$
\begin{aligned}
w_{y}= & \left(s+\theta_{y}\right) w+\left(\alpha+t_{y}\right)\left(z_{y}+s z\right) \\
& +\left(c_{x}+c \theta_{x}+q+s_{y}+c t-s^{2}-s \theta_{y}\right)\left(z_{x}+t z\right)+C z,
\end{aligned}
$$

where

$$
C=\left(c p+q_{x}-\alpha_{y}+\alpha \theta_{y}\right)-\left(c_{x}+c \theta_{x}\right) t-c t^{2} .
$$

The required condition for the vector $w_{y}$ is $C=0$; namely,

$$
c t^{2}+\left(c_{x}+c \theta_{x}\right) t-\left(c p+q_{x}-\alpha_{y}+\alpha \theta_{y}\right)=0 .
$$

Hence the envelope surfaces are given by solving two equations, (11.3) and (11.4); generally, we have four solutions.

We denote the discriminants of both equations (11.3) and (11.4) by $\Delta_{1}$ and $\Delta_{2}$, respectively. They are given as follows, in view of integrability:

$$
\Delta_{1}=b_{y}^{2}-2 b b_{y y}-2 b^{2} M, \quad \Delta_{2}=c_{x}^{2}-2 c c_{x x}-2 c^{2} L .
$$

Referring to (2.10), we can see that these discriminants are the same as those defined by (2.19). As we have not assumed that $\theta$ is constant, we need to replace $p$ and $q$ in (2.10) by $p-\theta_{x x} / 2+\theta_{x}^{2} / 4+b \theta_{x} / 2$ and $q-\theta_{y y} / 2+\theta_{y}^{2} / 4+c \theta_{y} / 2$.

Note that

$$
\Delta_{1 y}=-2 b\left(b_{y y y}+2 M b_{y}+b M_{y}\right), \quad \Delta_{2 x}=-2 c\left(c_{x x x}+2 L c_{x}+c L_{x}\right),
$$


hence the third integrability condition of (2.9) implies that

$$
c \Delta_{1 y}=b \Delta_{2 x},
$$

and, conversely, this identity is equivalent to the third integrability condition, provided that $b c \neq 0$.

To keep the notation $P$ and $Q$ defined in (2.18), in accordance with the case where $\theta$ is not necessarily constant, we need to change the formulas to

$$
P=p+\frac{b_{y}}{2}-\frac{c_{x x}}{2 c}+\frac{c_{x}^{2}}{4 c^{2}}-\frac{\theta_{x x}}{2}+\frac{1}{4} \theta_{x}^{2}+\frac{b \theta_{y}}{2}
$$

and

$$
Q=q+\frac{c_{x}}{2}-\frac{b_{y y}}{2 b}+\frac{b_{y}^{2}}{4 b^{2}}-\frac{\theta_{y y}}{2}+\frac{1}{4} \theta_{y}^{2}+\frac{c \theta_{x}}{2} .
$$

We refer to (2.11). For the benefit of later use, we introduce the notation $\sigma$ and $\tau$ by

$$
\sigma^{2}=\frac{\Delta_{1}}{4 b^{2}}=Q, \quad \tau^{2}=\frac{\Delta_{2}}{4 c^{2}}=P
$$

we refer to (2.24). Then

$$
s=-\frac{b_{y}}{2 b}-\frac{1}{2} \theta_{y} \pm \sigma, \quad t=-\frac{c_{x}}{2 c}-\frac{1}{2} \theta_{x} \pm \tau
$$

and the Demoulin transform $w$ is written as

$$
\begin{aligned}
w= & z_{x y}+s z_{x}+t z_{y}+\left(s t-\frac{1}{2} b c\right) z \\
= & z_{x y}-\left(\frac{b_{y}}{2 b}+\frac{1}{2} \theta_{y}\right) z_{x}-\left(\frac{c_{x}}{2 c}+\frac{1}{2} \theta_{y}\right) z_{y} \\
& +\left(\left(\frac{b_{y}}{2 b}+\frac{1}{2} \theta_{y}\right)\left(\frac{c_{x}}{2 c}+\frac{1}{2} \theta_{x}\right)-\frac{1}{2}\left(b c+\theta_{x y}\right)\right) z \\
& +\sigma\left(z_{x}-\left(\frac{c_{x}}{2 c}+\frac{1}{2} \theta_{x}\right) z\right)+\tau\left(z_{y}-\left(\frac{b_{y}}{2 b}+\frac{1}{2} \theta_{x}\right) z\right)+\sigma \tau z .
\end{aligned}
$$

We remark that this reduces to (2.25) when $\theta$ is constant. In terms of $\sigma$ and $\tau$, the formula in Lemma 2.12 can be written as follows:

$$
b\left(\sigma^{2}\right)_{x}=\left(b \kappa_{1}\right)_{y}, \quad c\left(\tau^{2}\right)_{y}=\left(c \kappa_{2}\right)_{x},
$$

where $\kappa_{1}$ and $\kappa_{2}$ are defined in (2.17). 


\subsection{Demoulin lines}

Here we look at the Demoulin transforms from a different point of view, by repeating the computation given in Section 2.1 in part. For simplicity, we assume that $\theta$ is constant in this section.

We recall that the dual coordinate system $\left(p^{0}, p^{1}, p^{2}, p^{3}\right)$ was defined by

$$
P=p^{0} z+p^{1} z_{x}+p^{2} z_{y}+p^{3} z_{x y}
$$

Relative to this coordinate system, the Lie quadric is defined by the equation $E=0$, where

$$
E:=p^{0} p^{3}-p^{1} p^{2}+\alpha\left(p^{3}\right)^{2}, \quad \alpha=\frac{1}{2} b c .
$$

We look for the characteristic points of the family of quadrics parametrized by $x$. They are defined by the equations $E=0$ and $E_{x}=0$. To compute $E_{x}$, we need variation formulas of the homogeneous coordinates $p^{i}$ when the point $P$ remains fixed.

As we have

$$
\begin{aligned}
P_{x}= & \left(p_{x}^{0}+p^{1} p+p^{3}\left(p_{y}+b q\right)\right) z+\left(p^{0}+p_{x}^{1}+p^{3} b c\right) z_{x} \\
& +\left(b p^{1}+p_{x}^{2}+p^{3}\left(b_{y}+p\right)\right) z_{y}+\left(p^{2}+p_{x}^{3}\right) z_{x y}, \\
P_{y}= & \left(p_{y}^{0}+p^{2} q+p^{3}\left(q_{x}+c p\right)\right) z+\left(c p^{2}+p_{y}^{1}+p^{3}\left(c_{x}+q\right)\right) z_{x} \\
& +\left(p^{0}+p_{y}^{2}+p^{3} b c\right) z_{x}+\left(p^{1}+p_{y}^{3}\right) z_{x y},
\end{aligned}
$$

if we assume the point $P$ remains fixed, then the coordinates $p^{i}$ vary by the following rules:

$$
\begin{gathered}
d p^{0}=-\left(p^{1} p+p^{3}\left(p_{y}+b q\right)\right) d x-\left(p^{2} q+p^{3}\left(q_{x}+c p\right)\right) d y \\
d p^{1}=-\left(p^{0}+p^{3} b c\right) d x-\left(c p^{2}+p^{3}\left(c_{x}+q\right)\right) d y \\
d p^{2}=-\left(b p^{1}+p^{3}\left(b_{y}+p\right)\right) d x-\left(p^{0}+p^{3} b c\right) d y \\
d p^{3}=-p^{2} d x-p^{1} d y
\end{gathered}
$$

Now it is easy to see that

$$
E_{x}=b\left(p^{1}\right)^{2}+b_{y} p^{1} p^{3}-\left(b_{y}+b q-\alpha_{x}\right)\left(p^{3}\right)^{2},
$$

and that the equation $E_{x}=0$ is nothing but the homogeneous form of equation (11.3). Any point satisfying the equation lies on the lines defined by the equations

$$
f:=p^{1}-s p^{3}=0, \quad g:=p^{0}-s p^{2}+\alpha p^{3},
$$


where $s$ is one of the solutions of (11.3). Similarly, for the family parametrized by $y$, we get the lines

$$
p^{2}-t p^{3}=0, \quad p^{0}-t p^{1}+\alpha p^{3}=0,
$$

where $t$ is one of the solutions of (11.4). Thus we get four distinguished lines on the Lie quadric, which were called the Demoulin lines.

Let us consider the line congruence consisting of Demoulin lines for each fixed choice of $s$ or $t$, which we call the Demoulin congruence.

We want to find the focal points of the Demoulin congruence. At such a point, we have a direction along which $d f=0$ and $d g=0$ hold. By computation, we see that

$$
d f=-\left(s_{x}+\alpha\right) p^{3} d x-\left(c p^{2}+\left(c_{x}+q+s_{y}-s^{2}\right) p^{3}\right) d y
$$

and

$$
d g=-\left(s_{x}+\alpha\right) p^{2} d x+\left(\left(s^{2}-q-s_{y}\right) p^{2}+\left(\alpha_{y}-q_{x}-c p\right) p^{3}\right) d y .
$$

Hence, the direction is determined so that both $d f$ and $d g$ are proportional, i.e. when the following identity holds:

$$
\left(s_{x}+\alpha\right)\left\{c\left(p^{2}\right)^{2}+c_{x} p^{2} p^{3}+\left(\alpha_{y}-q_{x}-c p\right)\left(p^{3}\right)^{2}\right\}=0 .
$$

As the focal surface is not determined if $s_{x}+\alpha=0$, we assume in the following that $s_{x}+\alpha \neq 0$. Then the condition above is the same as the condition $E_{y}=0$. Thus, we have seen that the Demoulin congruence is stationary at the points where the line in one family meets the line in the other family.

\subsection{Demoulin congruences}

We compute the asymptotic directions of the Demoulin transforms. The induced conformal form $\omega$ on the surface is determined by the identity

$$
w \wedge w_{x} \wedge w_{y} \wedge\left(w_{x x} d x^{2}+2 w_{x y} d x d y+w_{y y} d y^{2}\right)=\omega z \wedge z_{x} \wedge z_{y} \wedge z_{x y} .
$$

If we write $\omega=A d x^{2}+2 B d x d y+C d y^{2}$, then a computation using the integrability conditions (2.9) gives the formulas

$$
\begin{aligned}
A= & \rho\left(s_{x}+\alpha\right)\left(2 \frac{(c t)_{x}}{c}+\frac{c_{x x}}{c}\right), \quad C=\rho\left(t_{y}+\alpha\right)\left(2 \frac{(b s)_{y}}{b}+\frac{b_{y y}}{b}\right), \\
B= & \rho\left(s_{x}+\alpha\right)\left(t_{y}+\alpha\right)-\rho\left(b s+\frac{1}{2} b_{y}\right)\left(c t+\frac{1}{2} c_{x}\right) \\
& +\rho\left(s_{y}+\frac{b_{y}}{b} s+\frac{b_{y y}}{2 b}\right)\left(t_{x}+\frac{c_{x}}{c} t+\frac{c_{x x}}{2 c}\right),
\end{aligned}
$$


where

$$
\rho=\left(b_{y}+b s+t_{x}+p-t^{2}\right)\left(c_{x}+s_{y}+c q t-s^{2}\right)-\left(s_{x}+\alpha\right)\left(t_{y}+\alpha\right) .
$$

If $\rho=0$, then the Demoulin transform does not make a surface. Hence, we assume that $\rho \neq 0$ in the following. By definitions (11.8) and (11.9), we have

$$
\begin{gathered}
s_{x}+\alpha=\kappa_{1}+\sigma_{x}, \quad t_{y}+\alpha=\kappa_{2}+\tau_{y}, \\
b s+\frac{1}{2} b_{y}=b \sigma, \quad c t+\frac{1}{2} c_{x}=c \tau .
\end{gathered}
$$

Hence, we can conclude that the conformal structure of the Demoulin transform is defined by the form

$$
\omega=\left(\left(\kappa_{1}+\sigma_{x}\right) d x+\frac{(b \sigma)_{y}}{b} d y\right)\left(\frac{(c \tau)_{x}}{c} d x+\left(\kappa_{2}+\tau_{y}\right) d y\right)-b c \sigma \tau d x d y .
$$

We now define the non-degeneracy for a Demoulin transform by

$$
\rho \sigma \tau\left(\kappa_{1}+\sigma_{x}\right)\left(\kappa_{2}+\tau_{y}\right) \neq 0 .
$$

As

$$
\left(\sigma^{2}\right)_{x}=(\log b)_{y} \kappa_{1}+\left(\kappa_{1}\right)_{y}, \quad\left(\tau^{2}\right)_{y}=(\log c)_{x} \kappa_{2}+\left(\kappa_{2}\right)_{x}
$$

by (11.10), we must have $\kappa_{1} \kappa_{2} \neq 0$ under the assumption of non-degeneracy.

Now we can state the following theorem.

THEOREM 11.1. (Finikoff [Fi30]) Assume that the Demoulin transform is nondegenerate. Then the Demoulin congruence is a W-congruence if and only if the original surface is projectively minimal.

Proof. For a fixed $s$, consider a Demoulin congruence joining two Demoulin transforms corresponding to two values of $t$. In terms of $\sigma$, one corresponds to the value $\sigma$ and the other corresponds to $-\sigma$. As the asymptotic directions for each transform are defined by the equation $\omega=0$, in order that the congruence be a $W$-congruence, it is necessary and sufficient that the equation $\omega=0$ for $\sigma$ and the equation $\omega=0$ for $-\sigma$ define the same direction. Then, taking into account the non-degeneracy defined above, we can see that

$$
(b \sigma)_{y}=0 \quad \text { and } \quad(c \tau)_{x}=0 .
$$

To complete the proof, it is enough to see that this condition is equivalent to (2.40) for projective minimality. 


\subsection{An explicit form of Demoulin frames}

This section aims at getting a detailed form of a Demoulin transform.

We recall the notation of a non-degenerate surface $z(x, y)$ :

$$
z_{x x}=\theta_{x} z_{x}+b z_{y}+p z, \quad z_{y y}=c z_{x}+\theta_{y} z_{y}+q z .
$$

Differentiating these equations once, we get

$$
\begin{aligned}
& z_{x x y}=\theta_{x} z_{x y}+\left(b c+\theta_{x y}\right) z_{x}+\left(b \theta_{y}+b_{y}+p\right) z_{y}+\left(b q+p_{y}\right) z, \\
& z_{x y y}=\theta_{y} z_{x y}+\left(c_{x}+c \theta_{x}+q\right) z_{x}+\left(b c+\theta_{x y}\right) z_{y}+\left(c p+q_{x}\right) z .
\end{aligned}
$$

We introduce the notation

$$
\lambda=b \sigma+\frac{(c \tau)_{x}}{c}, \quad \lambda^{\prime}=c \tau+\frac{(b \sigma)_{y}}{b}, \quad \mu=\alpha+s_{x}, \quad \mu^{\prime}=\alpha+t_{y},
$$

and we remark that the following identities hold:

$$
\sigma(b \sigma)_{y}=\tau(c \tau)_{x}, \quad b \sigma \lambda^{\prime}=c \tau \lambda .
$$

We define vectors $Y, Z$, and $X$ by

$$
Y=t z+z_{x}, \quad Z=s z+z_{y}, \quad X=z_{x y}+s z_{x}+t z_{y}+(s t-\alpha) z .
$$

Then, from what we have shown, we get the following Pfaff equation:

$$
d\left(\begin{array}{l}
z \\
Y \\
Z \\
X
\end{array}\right)=\omega\left(\begin{array}{l}
z \\
Y \\
Z \\
X
\end{array}\right),
$$

where

$$
\omega=\left(\begin{array}{cccc}
-(t d x+s d y) & d x & d y & 0 \\
(\lambda-2 b \sigma) d x+\mu^{\prime} d y & \left(\theta_{x}+t\right) d x-s d y & b d x & d y \\
\mu d x+\left(\lambda^{\prime}-2 c \tau\right) d y & c d y & -t d x+\left(\theta_{y}+s\right) d y & d x \\
0 & \mu d x+\lambda^{\prime} d y & \lambda d x+\mu^{\prime} d y & \left(\theta_{x}+t\right) d x+\left(\theta_{y}+s\right) d y
\end{array}\right) .
$$

The integrability condition $d \omega=\omega \wedge \omega$ can be checked by using the identities given above and the additional identities

$$
\mu_{x}^{\prime}-\lambda_{y}=\mu^{\prime}\left(2 t+\theta_{x}\right)-b \lambda^{\prime}, \quad \mu_{y}-\lambda_{x}^{\prime}=\mu\left(2 s+\theta_{y}\right)-c \lambda .
$$

Provided that $R:=\lambda \lambda^{\prime}-\mu \mu^{\prime} \neq 0$, the vector $X$ defines a surface. Considering the Pfaff equation above, it is easy to see that the conformal structure on $X$ is given by the 2-form

$$
b \mu(c \tau)_{x} d x^{2}+b c\left(\lambda \lambda^{\prime}+\mu \mu^{\prime}-b \sigma \lambda^{\prime}-c \tau \lambda\right) d x d y+c(b \sigma)_{y} d y^{2} .
$$


The above coframe $\omega$ satisfies the required condition for the frame $\{z, Y, Z, X\}$ to be a Demoulin frame defined in Section 2.4, up to scalar multiplication. The invariants are seen to be as follows:

$$
\begin{gathered}
\omega^{1}=d x, \quad \omega^{2}=d y, \quad h_{111}=-2 b, \quad h_{112}=h_{122}=0, \quad h_{222}=-2 c, \\
\left(p_{i j}\right)=\left(\begin{array}{cc}
\lambda-2 b \sigma & \mu^{\prime} \\
\mu & \lambda^{\prime}-2 c \tau
\end{array}\right), \quad\left(q_{i j}\right)=\left(\begin{array}{ll}
\lambda & \mu^{\prime} \\
\mu & \lambda^{\prime}
\end{array}\right) \\
\left(L_{i j}\right)=\left(\begin{array}{cc}
2 b \sigma & 0 \\
0 & 2 c \tau
\end{array}\right) .
\end{gathered}
$$

Now we assume that the surface $z$ is projectively minimal, i.e. $(b \sigma)_{y}=0$ and $(c \tau)_{x}=0$. In this case, we simply get

$$
\lambda=b \sigma, \quad \lambda^{\prime}=c \tau,
$$

and, provided that $R \neq 0$, the induced conformal structure on the surface $X$ is the same as that on $z$. The system of equations defining $X$ is given as follows:

$$
X_{x x}=\bar{\theta}_{x} X_{x}+\bar{b} X_{y}+\bar{p} X, \quad X_{y y}=\bar{c} X_{x}+\bar{\theta}_{y} X_{y}+\bar{q} X .
$$

To simplify notation, we set

$$
v=\theta_{x}+t, \quad v^{\prime}=\theta_{y}+s .
$$

Then, we can see that

$$
\begin{gathered}
\bar{\theta}_{x}=\theta_{x}+\frac{R_{x}}{R}, \quad \bar{\theta}_{y}=\theta_{y}+\frac{R_{y}}{R}, \\
\bar{b}=\frac{\mu}{\mu^{\prime}} b+\frac{R \lambda_{x}-R_{x} \lambda}{R \mu^{\prime}}, \quad \bar{c}=\frac{\mu^{\prime}}{\mu} c+\frac{R \lambda_{y}^{\prime}-R_{y} \lambda^{\prime}}{R \mu}, \\
\bar{p}=v_{x}+v^{2}+\lambda-v \bar{\theta}_{x}-v^{\prime} \bar{b} \\
\bar{q}=v_{y}^{\prime}+v^{\prime 2}+\lambda^{\prime}-v^{\prime} \bar{\theta}_{y}-v \bar{c} .
\end{gathered}
$$

We also have expressions

$$
\begin{gathered}
\bar{b}=\frac{1}{R}\left(\lambda \mu(v+t)+\lambda \mu_{x}-\lambda_{x} \mu-b \mu^{2}\right), \\
\bar{c}=\frac{1}{R}\left(\lambda^{\prime} \mu^{\prime}\left(v^{\prime}+s\right)+\lambda^{\prime} \mu_{y}^{\prime}-\lambda_{y}^{\prime} \mu^{\prime}-c \mu^{\prime 2}\right) .
\end{gathered}
$$

Let us normalize the frame ${ }^{\mathrm{t}}(X, Y, Z, z)$. We know

$$
d\left(\begin{array}{l}
X \\
Y \\
Z \\
z
\end{array}\right)=\left(\begin{array}{cccc}
\rho & \mu d x+\lambda^{\prime} d y & \lambda d x+\mu^{\prime} d y & 0 \\
d y & v d x-s d y & b d x & -\lambda d x+\mu^{\prime} d y \\
d x & c d y & -t d x+v^{\prime} d y & \mu d x-\lambda^{\prime} d y \\
0 & d x & d y & -t d x-s d y
\end{array}\right)\left(\begin{array}{l}
X \\
Y \\
Z \\
z
\end{array}\right)
$$


where $\rho=\left(\theta_{x}+t\right) d x+\left(\theta_{y}+s\right) d y$. We define new vectors

$$
\bar{z}=-R z, \quad \bar{Y}=\mu Y+\lambda Z, \quad \bar{Z}=\lambda^{\prime} Y+\mu^{\prime} Z .
$$

Relative to the frame $f={ }^{\mathrm{t}}(X, \bar{Y}, \bar{Z}, \bar{z})$, we get $d f=\Omega f$, where

$$
\Omega=\left(\begin{array}{cccc}
\rho & d x & d y & 0 \\
\lambda d x+\mu d y & \delta d x+v^{\prime} d y & \bar{b} d x & d y \\
\mu^{\prime} d x+\lambda^{\prime} d y & \bar{c} d y & v d x+\delta^{\prime} d y & d x \\
0 & \mu^{\prime} d x-\lambda^{\prime} d y & -\lambda d x+\mu d y & \left(R_{x} / R-t\right) d x+\left(R_{y} / R-s\right) d y
\end{array}\right),
$$

and where

$$
\begin{gathered}
\delta=\left(-\mu^{\prime} \mu_{x}+\lambda^{\prime} \lambda_{x}-\mu \mu^{\prime} v-\lambda \lambda^{\prime} t+\mu \lambda^{\prime} b\right) / R, \\
\delta^{\prime}=\left(-\mu \mu_{y}^{\prime}+\lambda \lambda_{y}^{\prime}-\mu \mu^{\prime} v-\lambda \lambda^{\prime} s+\mu^{\prime} \lambda c\right) / R, \\
\bar{b}=\left(\lambda \mu_{x}-\mu \lambda_{x}+\lambda \mu v-\mu^{2} b+\lambda \mu t\right) / R, \\
\bar{c}=\left(\lambda^{\prime} \mu_{y}^{\prime}-\mu^{\prime} \lambda_{y}^{\prime}+\lambda^{\prime} \mu^{\prime} v^{\prime}-\mu^{\prime 2} c+\lambda^{\prime} \mu^{\prime} s\right) / R .
\end{gathered}
$$

The frame $f$ is the Demoulin frame of the Demoulin transform with $\Omega$ as its coframe when the original surface is projectively minimal.

Remark 11.2. The contents in this section were originally given by [Fi30] and [May32] ([L42] is also helpful). We also refer the reader to [Fi32, Fi59].

\section{An intrinsic description of Demoulin transforms of projectively minimal surfaces}

In the previous section we have seen that projectively minimal surfaces enjoy a special feature relative to Demoulin transforms. Referring to the intrinsic formulation of projectively minimal surface in Section 2.7, we compute normalized frames of Demoulin transforms and then get the formula of the second Demoulin transforms. Relying on explicit forms of the second Demoulin transforms, we reprove the result by Mayer and $\mathrm{Su}$ that a projectively minimal surface generally yields nine second Demoulin transforms. As an example, we explicitly give the second Demoulin transforms among the coincidence surfaces.

\subsection{The normalized frame of a Demoulin transform}

Let us recall the notation defined in Section 2.4: $e$ denotes a Demoulin frame and $\omega$ its coframe. Let $p$ and $q$ denote the matrices given in (2.20) and set $P=\operatorname{det} p$. 
We assume now that the surface is projectively minimal and that $P \neq 0$ so that every Demoulin transform defines a surface. Then by Proposition 2.22 we have the following expressions:

$$
\begin{gathered}
\left(p_{i j}\right)=\left(\begin{array}{ll}
p_{11} & p_{12} \\
p_{21} & p_{22}
\end{array}\right), \quad\left(q_{i j}\right)=\left(\begin{array}{cc}
-p_{11} & p_{12} \\
p_{21} & -p_{22}
\end{array}\right), \\
\left(\ell_{i j}\right)=\left(\begin{array}{cc}
-2 p_{11} & 0 \\
0 & -2 p_{22}
\end{array}\right) .
\end{gathered}
$$

The frame $\tilde{e}=\left(e_{3}, e_{1}, e_{2}, e_{0}\right)$ is associated with the Demoulin transform $e_{3}$, and the coframe defined by $d \tilde{e}=\tilde{\omega} \tilde{e}$ has the form

$$
\tilde{\omega}=\left(\begin{array}{cccc}
\omega_{3}^{3} & p_{21} \omega^{1}-p_{22} \omega^{2} & -p_{11} \omega^{1}+p_{12} \omega^{2} & 0 \\
\omega^{2} & \omega_{1}^{1} & b \omega^{1} & p_{11} \omega^{1}+p_{12} \omega^{2} \\
\omega^{1} & c \omega^{2} & \omega_{2}^{2} & p_{21} \omega^{1}+p_{22} \omega^{2} \\
0 & \omega^{1} & \omega^{2} & \omega_{0}^{0}
\end{array}\right) .
$$

This is not normalized, and in order to get a normalized frame, we need a slight change of the frame as follows:

$$
\begin{gathered}
\bar{e}_{0}=\delta e_{3}, \quad \bar{e}_{1}=\delta\left(p_{21} e_{1}-p_{11} e_{2}\right), \\
\bar{e}_{2}=\delta\left(p_{12} e_{2}-p_{22} e_{1}\right), \quad \bar{e}_{3}=-\delta P e_{0},
\end{gathered}
$$

where $\delta=1 / \sqrt{P}$. Then, the normalized coframe defined by $d \bar{e}=\overline{\omega e}$ has the form

$$
\bar{\omega}=\left(\begin{array}{cccc}
d \log \delta+\omega_{3}^{3} & \omega^{1} & \omega^{2} & 0 \\
p_{21} \omega^{2}-p_{11} \omega^{1} & \bar{\omega}_{1}^{1} & \bar{\omega}_{1}^{2} & \omega^{2} \\
p_{12} \omega^{1}-p_{22} \omega^{2} & \bar{\omega}_{2}^{1} & \bar{\omega}_{2}^{2} & \omega^{1} \\
0 & p_{12} \omega^{1}+p_{22} \omega^{2} & p_{11} \omega^{1}+p_{21} \omega^{2} & \omega_{0}^{0}-d \log \delta
\end{array}\right),
$$

where

$$
\begin{aligned}
P \bar{\omega}_{1}^{1}= & -d P / 2-p_{12} d p_{21}+p_{22} d p_{11}-p_{12} p_{21} \omega_{1}^{1}+p_{11} p_{12} \omega_{2}^{1}-p_{22} p_{21} \omega_{1}^{2} \\
& +p_{11} p_{22} \omega_{2}^{2}, \\
P \bar{\omega}_{1}^{2}= & -p_{11} d p_{21}+p_{21} d p_{11}-p_{11} p_{21} \omega_{1}^{1}+\left(p_{11}\right)^{2} \omega_{2}^{1}-\left(p_{21}\right)^{2} \omega_{1}^{2}+p_{11} p_{21} \omega_{2}^{2}, \\
P \bar{\omega}_{2}^{1}= & p_{12} d p_{22}-p_{22} d p_{12}+p_{12} p_{22} \omega_{1}^{1}-\left(p_{12}\right)^{2} \omega_{2}^{1}+\left(p_{22}\right)^{2} \omega_{1}^{2}-p_{12} p_{22} \omega_{2}^{2}, \\
P \bar{\omega}_{2}^{2}= & -d P / 2+p_{11} d p_{22}-p_{21} d p_{12}+p_{11} p_{22} \omega_{1}^{1}-p_{11} p_{12} \omega_{2}^{1}+p_{21} p_{22} \omega_{1}^{2} \\
& -p_{12} p_{21} \omega_{2}^{2} .
\end{aligned}
$$


We note that the conditions $\bar{\omega}_{0}^{0}+\bar{\omega}_{3}^{3}=0, \bar{\omega}_{1}^{1}+\bar{\omega}_{2}^{2}=0$ and $\bar{\omega}_{3}^{0}=0$ are satisfied as required for normalization. The associated invariants $\bar{h}, \bar{p}, \bar{q}$ and $\bar{\ell}$ are

$$
\begin{gathered}
\bar{h}=\left(\begin{array}{ll}
0 & 1 \\
1 & 0
\end{array}\right), \quad \bar{p}=\left(\begin{array}{cc}
-p_{11} & p_{21} \\
p_{12} & -p_{22}
\end{array}\right), \\
\bar{q}=\left(\begin{array}{ll}
p_{11} & p_{21} \\
p_{12} & p_{22}
\end{array}\right), \quad \bar{\ell}=\left(\begin{array}{cc}
2 p_{11} & 0 \\
0 & 2 p_{22}
\end{array}\right) .
\end{gathered}
$$

These formulas say that, for the Demoulin transform $e_{3}, p_{11}$ and $p_{22}$ are changed into their negatives and $p_{12}$ and $p_{21}$ are interchanged. In particular, for Godeaux-Rozet surfaces, we have the following corollary in view of Remark 2.9.

COROLlary 12.1. Any Demoulin transform of a Godeaux-Rozet surface is also a Godeaux-Rozet surface.

The cubic invariants $\bar{b}$ and $\bar{c}$ are defined by the formulas $\bar{h}_{111}=-2 \bar{b}$ and $\bar{h}_{222}=-2 \bar{c}$, and we see that

$$
\bar{\omega}_{1}^{2}=\bar{b} \omega^{1}, \quad \bar{\omega}_{2}^{1}=\bar{c} \omega^{2} .
$$

We now calculate the cubic invariants explicitly.

We define covariant derivatives of $p_{i j}$ by

$$
d p_{i j}-\sum_{k} p_{i k} \omega_{j}^{k}-\sum_{k} p_{k j} \omega_{i}^{k}+2 p_{i j} \omega_{0}^{0}=\sum_{k} p_{i j, k} \omega^{k} .
$$

LEMMA 12.2. We have $p_{11,2}=p_{12,1}=0$ and $p_{21,2}=p_{22,1}=0$.

Proof. The differentiation of $\omega_{i}^{0}=p_{i j} \omega^{j}$ implies that $d \omega_{i}^{0}-d p_{i j} \wedge \omega^{j}-p_{i j} d \omega^{j}=0$, and the left-hand side is equal to $\omega^{j} \wedge\left(d p_{i j}-p_{i k} \omega_{j}^{k}-p_{k j} \omega_{i}^{k}+2 p_{i j} \omega_{0}^{0}\right)$. Hence, we have $p_{i j, k}=p_{i k, j}$.

Next we differentiate $\omega_{1}^{0}=\sum p_{1 j} \omega^{j}$ and get

$$
d p_{11} \wedge \omega^{1}+d p_{12} \wedge \omega^{2}=2 p_{11}\left(\omega_{1}^{1}-\omega_{0}^{0}\right) \wedge \omega^{1}-2 p_{12} \omega_{0}^{0} \wedge \omega^{2}+b p_{22} \omega^{1} \wedge \omega^{2} .
$$

The differentiation of $\omega_{3}^{2}=\sum q_{1 j} \omega^{j}$ gives

$$
d q_{11} \wedge \omega^{1}+d q_{12} \wedge \omega^{2}=2 q_{11}\left(\omega_{1}^{1}-\omega_{0}^{0}\right) \wedge \omega^{1}-2 q_{12} \omega_{0}^{0} \wedge \omega^{2}-b q_{22} \omega^{1} \wedge \omega^{2} .
$$

As $q_{11}=-p_{11}, q_{12}=p_{12}$ and $q_{22}=-p_{22}$, the latter identity means that

$$
-d p_{11} \wedge \omega^{1}+d p_{12} \wedge \omega^{2}=-2 p_{11}\left(\omega_{1}^{1}-\omega_{0}^{0}\right) \wedge \omega^{1}-2 p_{12} \omega_{0}^{0} \wedge \omega^{2}+b p_{22} \omega^{1} \wedge \omega^{2} .
$$


Hence, we have

$$
\begin{gathered}
d p_{11} \wedge \omega^{1}=2 p_{11}\left(\omega_{1}^{1}-\omega_{0}^{0}\right) \wedge \omega^{1} \\
d p_{12} \wedge \omega^{2}=b p_{22} \omega^{1} \wedge \omega^{2}-2 p_{12} \omega_{0}^{0} \wedge \omega^{2} .
\end{gathered}
$$

By a similar computation for the identities $\omega_{2}^{0}=p_{2 j} \omega^{j}$ and $\omega_{3}^{1}=q_{2 j} \omega^{j}$, we also have

$$
\begin{gathered}
d p_{22} \wedge \omega^{2}=-2 p_{22}\left(\omega_{1}^{1}+\omega_{0}^{0}\right) \wedge \omega^{2}, \\
d p_{21} \wedge \omega^{1}=-c p_{11} \omega^{1} \wedge \omega^{2}-2 p_{21} \omega_{0}^{0} \wedge \omega^{1} .
\end{gathered}
$$

We now insert $d p_{11}=p_{11,1} \omega^{1}+p_{11,2} \omega^{2}+2 p_{11} \omega_{1}^{1}+\left(p_{12}+p_{21}\right) \omega_{1}^{2}-2 p_{11} \omega_{0}^{0}$, which is one of the formulas in (12.5), into the first equation of (12.6) to get $p_{11,2}=0$ by using the property that $\omega_{1}^{2}=b \omega^{2}$ contains no term with $\omega^{1}$. The other claimed identities are similarly shown.

Now it is easy to compute $P \bar{\omega}_{1}^{2}$ defined in (12.4) by use of the $d p_{21}$ and $d p_{11}$ given in (12.5), and we get

$$
P \bar{\omega}_{1}^{2}=\left(p_{21} p_{11,1}-p_{11} p_{21,1}\right) \omega^{1}-P \omega_{1}^{2} .
$$

Hence, we have the following.

LEMMA 12.3. Let $P=\operatorname{det}(p)$ and let $\bar{b}$ and $\bar{c}$ denote the cubic invariants of the Demoulin transform. Then

$$
\begin{aligned}
P \bar{b} & =-P b+p_{21} p_{11,1}-p_{11} p_{21,1}, \\
P \bar{c} & =-P c+p_{12} p_{22,2}-p_{22} p_{12,2} .
\end{aligned}
$$

We assume in the following that $L_{11} L_{22} \neq 0$ so that we have four distinct Demoulin transforms. Let us recall the treatment in Section 2.4 again. Once we get a Demoulin frame $e$, the other Demoulin frames are represented by $g e$, where $g$ is the transformation of the form

$$
g=\left(\begin{array}{cccc}
1 & 0 & 0 & 0 \\
\rho^{2} & 1 & 0 & 0 \\
\rho^{1} & 0 & 1 & 0 \\
\rho^{1} \rho^{2} & \rho^{1} & \rho^{2} & 1
\end{array}\right)
$$

the values $\rho^{1}$ and $\rho^{2}$ are determined by the conditions

$$
b\left(\rho^{1}\right)^{2}+\ell_{11} \rho^{1}=0 \text { and } c\left(\rho^{2}\right)^{2}+\ell_{22} \rho^{2}=0 .
$$


We set

$$
t^{1}=-\ell_{11} / b, \quad t^{2}=-\ell_{22} / c .
$$

Then, $\rho^{1}$ takes the value 0 or $t^{1}$ and $\rho^{2}$ takes the value 0 or $t^{2}$.

We denote one of the new frames by $\tilde{e}=\left\{\tilde{e}_{0}, \tilde{e}_{1}, \tilde{e}_{2}, \tilde{e}_{3}\right\}$, where

$\tilde{e}_{0}=e_{0}, \quad \tilde{e}_{1}=\rho^{2} e_{0}+e_{1}, \quad \tilde{e}_{2}=\rho^{1} e_{0}+e_{2}, \quad \tilde{e}_{3}=\rho^{1} \rho^{2} e_{0}+\rho^{1} e_{1}+\rho^{2} e_{2}+e_{3}$, and then the new $\tilde{\ell}_{i j}$ are given by

$$
\tilde{\ell}_{11}=\ell_{11}+2 b \rho^{1}, \quad \tilde{\ell}_{22}=\ell_{22}+2 c \rho^{2} .
$$

The coframe $\tilde{\omega}, d \tilde{e}=\tilde{\omega} \tilde{e}$, is

$$
\tilde{\omega}=\left(\begin{array}{cccc}
\omega_{0}^{0}-\rho^{2} \omega^{1}-\rho^{1} \omega^{2} & \omega^{1} & \omega^{2} & 0 \\
\tilde{\omega}_{1}^{0} & \omega_{1}^{1}+\rho^{2} \omega^{1}-\rho^{1} \omega^{2} & \omega_{1}^{2} & \omega^{2} \\
\tilde{\omega}_{2}^{0} & \omega_{2}^{1} & \omega_{2}^{2}+\rho^{1} \omega^{2}-\rho^{2} \omega^{1} & \omega^{1} \\
0 & \tilde{\omega}_{3}^{1} & \tilde{\omega}_{3}^{2} & \omega_{3}^{3}+\rho^{2} \omega^{1}+\rho^{1} \omega^{2}
\end{array}\right) \text {, }
$$

where

$$
\begin{aligned}
& \tilde{\omega}_{1}^{0}=\omega_{1}^{0}-\rho^{1} \omega_{1}^{2}+d \rho^{2}-\left(\rho^{2}\right)^{2} \omega_{1}^{1}-\rho^{1} \omega_{1}^{2}+\rho^{2} \omega_{0}^{0}, \\
& \tilde{\omega}_{2}^{0}=\omega_{2}^{0}-\rho^{2} \omega_{2}^{1}+d \rho^{1}-\left(\rho^{1}\right)^{2} \omega^{2}-\rho^{1} \omega_{2}^{2}+\rho^{1} \omega_{0}^{0}, \\
& \tilde{\omega}_{3}^{1}=\omega_{3}^{1}+\rho^{2} \omega_{2}^{1}+d \rho^{1}-\left(\rho^{1}\right)^{2} \omega^{2}+\rho^{1} \omega_{1}^{1}-\rho^{1} \omega_{3}^{3}, \\
& \tilde{\omega}_{3}^{2}=\omega_{3}^{2}+\rho^{1} \omega_{1}^{2}+d \rho^{2}-\left(\rho^{2}\right)^{2} \omega_{1}+\rho^{2} \omega_{2}^{2}-\rho^{2} \omega_{3}^{3} .
\end{aligned}
$$

We remark that, for any choice of frame, the cubic invariants $b$ and $c$ remain the same.

We now assume that the surface is projectively minimal.

LEMMA 12.4. The derivations of $t^{1}=-\ell_{11} / b$ and $t^{2}=-\ell_{22} / c$ have the following expressions:

$$
\begin{aligned}
& d t^{1}+t^{1}\left(\omega_{0}^{0}+\omega_{1}^{1}\right)-\left(t^{1}\right)^{2} \omega^{2}=t_{, 1}^{1} \omega^{1}, \\
& d t^{2}+t^{2}\left(\omega_{0}^{0}+\omega_{2}^{2}\right)-\left(t^{2}\right)^{2} \omega^{1}=t_{, 2}^{2} \omega^{2},
\end{aligned}
$$

where $t_{, 1}^{1}$ and $t_{, 2}^{2}$ are thus defined.

Proof. The exterior derivative of $\omega_{3}^{2}-\omega_{1}^{0}=\ell_{11} \omega^{1}$ yields

$$
\left(d \ell_{11}+2 \ell_{11}\left(\omega_{0}^{0}-\omega_{1}^{1}\right)\right) \wedge \omega^{1}=0,
$$

and the exterior derivative of $\omega_{1}^{2}=b \omega^{1}$ yields

$$
d b \wedge \omega^{1}=-b\left(\omega_{0}^{0}-3 \omega_{1}^{1}\right) \wedge \omega^{1}-\ell_{11} \omega^{1} \wedge \omega^{2} .
$$


Hence, we have

$$
\left(b d \ell_{11}-\ell_{11} d b+b \ell_{11}\left(\omega_{0}^{0}+\omega_{1}^{1}\right)+\left(\ell_{11}\right)^{2} \omega^{2}\right) \wedge \omega^{1}=0
$$

from which, by dividing both sides by $b^{2}$, we get the first assertion. The second assertion is similarly proved.

By this lemma, the new coframe simplifies to

$$
\begin{array}{ll}
\tilde{\omega}_{1}^{0}=\omega_{1}^{0}-\rho^{1} \omega_{1}^{2}+t_{, 2}^{2} \omega^{2}, & \tilde{\omega}_{2}^{0}=\omega_{2}^{0}-\rho^{2} \omega_{2}^{1}+t_{, 1}^{1} \omega^{1}, \\
\tilde{\omega}_{3}^{1}=\omega_{3}^{1}+\rho^{2} \omega_{2}^{1}+t_{, 1}^{1} \omega^{1}, & \tilde{\omega}_{3}^{2}=\omega_{3}^{2}+\rho^{1} \omega_{1}^{2}+t_{, 2}^{2} \omega^{2} .
\end{array}
$$

Here we understand that $t_{, 1}^{1}=0$ when $\rho^{1}=0$ and $t_{, 2}^{2}=0$ when $\rho^{2}=0$.

Summing up the discussion above, we get the following proposition.

Proposition 12.5. Assume that the original surface is a non-ruled indefinite projectively minimal surface and that $\ell_{11} \ell_{22} \neq 0$. Let $e=\left\{e_{0}, e_{1}, e_{2}, e_{3}\right\}$ be one of the Demoulin frames. Then the four Demoulin frames are given as follows.

\begin{tabular}{ccccc}
\hline Type & $\rho^{1}$ & $\rho^{2}$ & Frame & Invariant $p$ \\
\hline 1 & 0 & 0 & $e_{0}, e_{1}, e_{2}, e_{3}$ & $\left(\begin{array}{ll}p_{11} & p_{12} \\
p_{21} & p_{22}\end{array}\right)$ \\
2 & $t^{1}$ & 0 & $e_{0}, e_{1}, e_{2}+t^{1} e_{0}, e_{3}+t^{1} e_{1}$ & $\left(\begin{array}{cc}-p_{11} & p_{12} \\
p_{21}^{\prime} & p_{22}\end{array}\right)$ \\
3 & 0 & $t^{2}$ & $e_{0}, e_{1}+t^{2} e_{0}, e_{2}, e_{3}+t^{2} e_{2}$ & $\left(\begin{array}{cc}p_{11} & p_{12}^{\prime} \\
p_{21} & -p_{22}\end{array}\right)$ \\
4 & $t^{1}$ & $t^{2}$ & $e_{0}, e_{1}+t^{2} e_{0}, e_{2}+t^{1} e_{0}, e_{3}+t^{1} e_{1}+t^{2} e_{2}+t^{1} t^{2} e_{0}\left(\begin{array}{cc}-p_{11} & p_{12}^{\prime} \\
p_{21}^{\prime} & -p_{22}\end{array}\right)$ \\
\hline
\end{tabular}

where

$$
p_{21}^{\prime}=p_{21}+t_{, 1}^{1}, \quad p_{12}^{\prime}=p_{12}+t_{, 2}^{2} .
$$

The following table gives the normalized frames and invariants for each of these Demoulin transforms; let $T i(1 \leq i \leq 4)$ denote the transform of type $i$ given in Lemma 12.5. Let $e^{i}=\left\{e_{0}^{i}, e_{1}^{i}, e_{2}^{i}, e_{3}^{i}\right\}$ denote the normalized frame for the transform $T i$. The matrix in the last column of the table below represents the invariants of the transformed surface, whose determinant is denoted by $P^{i}$. 


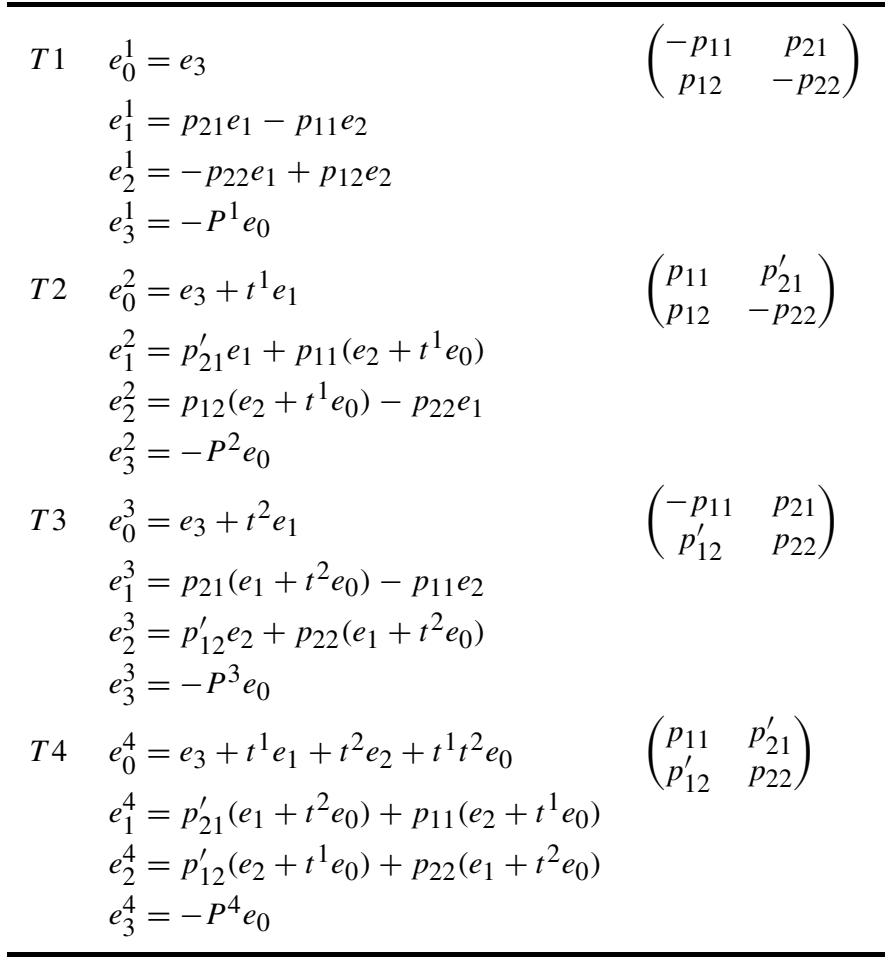

The above computation is valid even when $\ell_{11} \ell_{22}=0$ provided $b c \neq 0$. When both of $\ell_{11}$ and $\ell_{22}$ vanish, namely, when the surface is a Demoulin surface, we get only one surface. When $\ell_{11}=0$ and $\ell_{22} \neq 0$, namely when the surface is a Godeaux-Rozet surface, we need to set $t^{1}=0$ in the above computation and we get $t_{.1}^{1}=0$. Then, we see that $p_{21}^{\prime}=p_{21}$. As $p_{11}=0$ in this case, we get $T 1=T 3$ and $T 2=T 4$. Similarly when $\ell_{11} \neq 0$ and $\ell_{22}=0$, we get $T 1=T 3$ and $T 2=T 4$.

\subsection{Second Demoulin transforms}

The repeated use of the above procedure gives the second transforms, which are listed below. Let us denote by $b^{i}$ and $c^{i}$ the respective cubic invariants for $T i$ and we use the label $T i j$ for denoting the second transforms of $T i$, where the number $j$ is given by following the same rule as used for labeling $T i$. We remark that for these transforms to really define surfaces, we need to assume that $P^{i} \neq 0, b^{i} \neq 0$ and $c^{i} \neq 0$. 


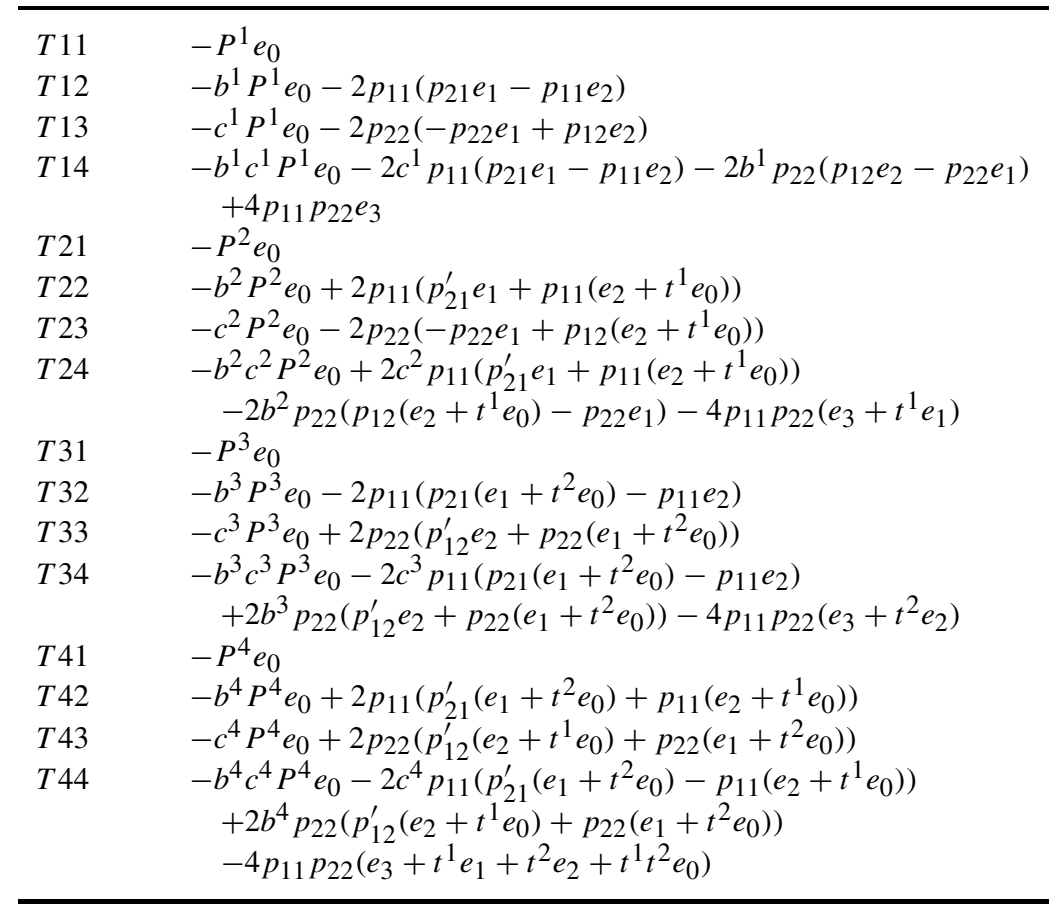

As is seen above, each Ti1 $(1 \leq i \leq 4)$ coincides with the original surface. Furthermore, we have the following.

Proposition 12.6. The following coincidences of surfaces hold: $T 13=T 23$, $T 12=T 32, T 22=T 42$ and $T 33=T 43$.

Proof. We prove the coincidence $T 13=T 23$. By definition,

$$
\begin{aligned}
T 13 & =-c^{1} P^{1} e_{0}-2 p_{22}\left(-p_{22} e_{1}+p_{12} e_{2}\right), \\
T 23 & =-c^{2} P^{2} e_{0}-2 p_{22}\left(-p_{22} e_{1}+p_{12}\left(e_{2}+t^{1} e_{0}\right)\right) \\
& =-\left(c^{2} P^{2}+2 p_{22} p_{12} t^{1}\right) e_{0}-2 p_{22}\left(-p_{22} e_{1}+p_{12} e_{2}\right) .
\end{aligned}
$$

Thus, it is enough to see that

$$
P^{1} c^{1}=P^{2} c^{2}+2 p_{22} p_{12} t^{1}=P^{2} c^{2}+4 p_{11} p_{22} p_{12} / b,
$$


where $P^{2}=-p_{11} p_{22}-p_{12} p_{21}^{\prime}$ is the determinant of the invariant matrix for $T 23$ :

$$
\left(\begin{array}{ll}
\tilde{p}_{11} & \tilde{p}_{12} \\
\tilde{p}_{21} & \tilde{p}_{22}
\end{array}\right)=\left(\begin{array}{cc}
p_{11} & p_{21}^{\prime} \\
p_{12} & -p_{22}
\end{array}\right)
$$

By Lemma 12.3,

$$
P^{1} c^{1}=-P^{1} c+p_{12} p_{22,2}-p_{22} p_{12,2}
$$

for the transform $T 13$ and

$$
P^{2} c^{2}=-P^{2} c+\tilde{p}_{12} \tilde{p}_{22,2}-\tilde{p}_{22} \tilde{p}_{12,2}
$$

for the transform $T 23$. The coframe for $T 23$ is, by (12.10),

$$
\tilde{\omega}=\left(\begin{array}{cccc}
\omega_{0}^{0}-t^{1} \omega^{2} & \omega^{1} & \omega^{2} & 0 \\
\tilde{\omega}_{1}^{0} & \omega_{1}^{1}-t^{1} \omega^{2} & \omega_{1}^{2} & \omega^{2} \\
\tilde{\omega}_{2}^{0} & \omega_{2}^{1} & \omega_{2}^{2}+t^{1} \omega^{2} & \omega^{1} \\
0 & \tilde{\omega}_{3}^{1} & \tilde{\omega}_{3}^{2} & \omega_{3}^{3}+t^{1} \omega^{2}
\end{array}\right) .
$$

By using this form,

$$
\begin{aligned}
\sum \tilde{p}_{22, k} \omega^{k} & =d \tilde{p}_{22}-\sum\left(\tilde{p}_{2 k}+\tilde{p}_{k 2}\right) \tilde{\omega}_{2}^{k}+2 \tilde{p}_{22} \tilde{\omega}_{0}^{0} \\
& =d p_{22}-\left(p_{21}+p_{12}+t_{, 1}^{1}\right) \omega_{2}^{1}-2 p_{22}\left(\omega_{2}^{2}+c^{1} \omega^{2}\right)+2 p_{22}\left(\omega_{0}^{0}-c^{1} \omega^{2}\right) \\
& =\sum p_{22, k} \omega^{k}-\left(c t_{, 1}^{1}+4 t^{1} p_{22}\right) \omega^{2}
\end{aligned}
$$

and

$$
\begin{aligned}
\sum \tilde{p}_{12, k} \omega^{k} & =d \tilde{p}_{12}-\tilde{p}_{11} \tilde{\omega}_{2}^{1}-\tilde{p}_{12}\left(\tilde{\omega}_{2}^{2}+\tilde{\omega}_{1}^{1}\right)-\tilde{p}_{22} \tilde{\omega}_{1}^{2}+2 \tilde{p}_{12} \tilde{\omega}_{0}^{0} \\
& =d p_{12}+p_{11} \omega_{2}^{1}-p_{12}\left(\omega_{2}^{2}+\omega_{1}^{1}\right)-p_{22} \omega_{1}^{2}+2 p_{12}\left(\omega_{0}^{0}-c^{1} \omega^{2}\right) \\
& =\sum p_{12, k} \omega^{k}+\left(2 p_{11} c-2 p_{12} t^{1}\right) \omega^{2} .
\end{aligned}
$$

Here, we used $\omega_{1}^{2}=b \omega^{2}, \omega_{2}^{1}=c \omega^{1}$, and $\omega_{1}^{1}+\omega_{2}^{2}=0$. Hence,

$$
\tilde{p}_{12} \tilde{p}_{22,2}-\tilde{p}_{22} \tilde{p}_{12,2}=p_{12} p_{22,2}-p_{22} p_{12,2}-c t_{, 1}^{1} p_{12}-2 c p_{11} p_{22}-2 t^{1} p_{12} p_{22} .
$$

Now it is easy to show (12.9) by using $t^{1}=2 p_{11} / b$. 
The arrangement of transforms can be illustrated as in the following diagram:

\begin{tabular}{|c|c|c|}
\hline \multicolumn{3}{|c|}{$T 32=T 12$} \\
\hline$T 34 \bullet$ & & - $T 14$ \\
\hline & * T1 & \\
\hline \multirow{2}{*}{$T 33=T 43 \circ$} & & $\circ T 13=T 23$ \\
\hline & * T2 & \\
\hline \multirow[t]{2}{*}{$T 44 \bullet$} & & - $T 24$ \\
\hline & & \\
\hline
\end{tabular}

where $\odot$ is the original surface, $*$ denotes the first Demoulin transforms, and $\circ$ and $\bullet$ denote the second Demoulin transforms.

We next consider the line congruence joining the original projectively minimal surface and four of the second transforms. We show the following.

PROPOSITION 12.7. The line congruence joining the original projectively minimal surface and each one of the surfaces T13, T12, T33 and T22 is a W-congruence, and these four surfaces are focal surfaces of each congruence.

Proof. We give a proof for $T 13$. Let us recall that the transform $T 1$ has a normalized frame

$$
e_{0}^{1}=e_{3}, \quad e_{1}^{1}=p_{21} e_{1}-p_{11} e_{2}, \quad e_{2}^{1}=-p_{22} e_{1}+p_{12} e_{2}, \quad e_{3}^{1}=-P^{1} e_{0}
$$

up to a scalar factor. The invariant matrix, which we denote by $\bar{p}$, is

$$
\bar{p}=\left(\begin{array}{ll}
\bar{p}_{11} & \bar{p}_{12} \\
\bar{p}_{21} & \bar{p}_{22}
\end{array}\right)=\left(\begin{array}{cc}
-p_{11} & p_{21} \\
p_{12} & -p_{22}
\end{array}\right) \text {. }
$$

Let $\bar{t}^{2}=-2 p_{22} / c^{1}$ be the value of $t^{2}$ for the transform $T 1$. Then, the normalized Demoulin frame for $T 1$ that is needed define $T 13$ is given by

$$
\tilde{e}_{0}=e_{0}^{1}, \quad \tilde{e}_{1}=e_{1}^{1}+\bar{t}^{2} e_{0}^{1}, \quad \tilde{e}_{2}=e_{2}^{1}, \quad \tilde{e}_{3}=e_{3}^{1}+\bar{t}^{2} e_{2}^{1}
$$

The corresponding invariant matrix is

$$
\tilde{p}=\left(\begin{array}{cc}
\tilde{p}_{11} & \tilde{p}_{12} \\
\tilde{p}_{21} & \tilde{p}_{22}
\end{array}\right)=\left(\begin{array}{cc}
\bar{p}_{11} & \bar{p}_{12}^{\prime} \\
\bar{p}_{21} & -\bar{p}_{22}
\end{array}\right),
$$


where $\bar{p}_{12}^{\prime}=\bar{p}_{12}+\bar{t}_{, 2}^{2}$ and $\bar{t}_{, 2}^{2}$ is defined by

$$
d \bar{t}^{2}+\bar{t}^{2}\left(\bar{\omega}_{0}^{0}-\bar{\omega}_{1}^{1}\right)-\left(\bar{t}^{2}\right)^{2} \omega^{1}=\bar{t}_{, 2}^{2} \omega^{2}
$$

relative to the connection form $\bar{\omega}$ in (12.3). Now we see that the normalized Demoulin frame of $T 13$ is given by

$$
f_{0}=\tilde{e}_{3}, \quad f_{1}=\tilde{p}_{21} \tilde{e}_{1}-\tilde{p}_{11} \tilde{e}_{2}, \quad f_{2}=-\tilde{p}_{22} \tilde{e}_{1}+\tilde{p}_{12} \tilde{e}_{2}, \quad f_{3}=-\tilde{P} \tilde{e}_{0},
$$

up to a multiplicative factor, where $\tilde{P}$ denotes the determinant of the matrix $\tilde{p}$. Then, we see that

$$
\begin{aligned}
& f_{1}=p_{12}\left(p_{21} e_{1}-p_{11} e_{2}+\bar{t}^{2} e_{3}\right)-\left(-p_{11}\right)\left(p_{12} e_{2}-p_{22} e_{1}\right), \\
& f_{2}=\left(p_{21}+\bar{t}_{, 2}^{2}\right)\left(p_{12} e_{2}-p_{22} e_{1}\right)-p_{22}\left(p_{21} e_{1}-p_{11} e_{2}+\bar{t}^{2} e_{3}\right) .
\end{aligned}
$$

Thus, we get

$$
p_{22} f_{1}+p_{12} f_{2}=\left(p_{11} p_{22}+p_{12} p_{21}+p_{12} \bar{t}_{, 2}^{2}\right)\left(-p_{22} e_{1}+p_{12} e_{2}\right) .
$$

This means that the line joining the original surface and $T 13$ is tangent to each surface. The congruence obtained is a $W$-congruence because each Demoulin transform preserves the conformal structure. Thus, we have Proposition 12.7.

Now let us set aside the long procedure above for a moment and give a summary. Given a (indefinite) projectively minimal non-ruled surface $z$ with a Demoulin frame $e$ and coframe $\omega$, we defined the Demoulin transform. We denoted by

$$
p=\left(\begin{array}{ll}
p_{11} & p_{12} \\
p_{21} & p_{22}
\end{array}\right)
$$

the invariant of the surface and assumed that $\operatorname{det}(p) \neq 0$ so that the transform really defines a surface, and we assumed that $p_{11} p_{22} \neq 0$ so that we get four distinct transforms. Then, each transform is projectively minimal. We now define a new surface $w$ by

$$
w=\frac{1}{2} \kappa z+p_{21} e_{1}-p_{11} e_{2} \quad \text { or } \quad w=\frac{1}{2} \kappa^{\prime} z-p_{22} e_{1}+p_{12} e_{2},
$$

where $\kappa$ and $\kappa^{\prime}$ are certain scalar functions determined by composing (12.10) and (12.11). Then the transform

$$
z \longmapsto w
$$

defines a W-congruence whose focal surfaces are both projectively minimal. By using the notation in Section 11.6, the new surface is

$$
w=\frac{1}{2} \kappa z+\mu z_{x}+\lambda z_{y} \quad \text { or } \quad w=\frac{1}{2} \kappa^{\prime} z+\lambda^{\prime} z_{x}+\mu^{\prime} z_{y},
$$

respectively. 
In Section 11.4 we defined a Demoulin congruence, and in Section 11.5 we proved that the Demoulin congruence is a $W$-congruence when the surface is projectively minimal. In the formulation of this section, this corresponds to the following fact. Let us consider the line congruence joining two surfaces $T 1=e_{3}$ and $T 2=e_{3}+t^{1} e_{1}$. The tangent plane of $T 1$ is spanned by the vectors $e_{1}^{1}=p_{21} e_{1}-p_{11} e_{2}$ and $e_{2}^{1}=$ $-p_{22} e_{1}+p_{12} e_{2}$, and the tangent plane of $T 2$ is spanned by $e_{1}^{2}=p_{21}^{\prime} e_{1}+p_{11}\left(e_{2}+t^{1} e_{0}\right)$ and $e_{2}^{2}=p_{12}\left(e_{2}+t^{1} e_{0}\right)-p_{22} e_{1}$. Hence, $-P^{1} e_{1}$ is included in the tangent plane of $T 1$ and $-P^{2} e_{1}$ is included in the tangent plane of $T 3$. As the line joining $T 1$ and $T 2$ is in the direction of the vector $e_{1}$, both surfaces $T 1$ and $T 2$ are focal surfaces of the congruence.

\subsection{Coincidence surfaces}

The surface defined by the system

$$
z_{x x}=z_{y}+\left(k x+k_{1}\right) z, \quad z_{y y}=z_{x}+\left(k y+k_{2}\right) z
$$

where $k, k_{1}$, and $k_{2}$ are constants, is called a coincidence surface. We refer the reader to Example 2.19. It is easy to check that this surface $z$ is projectively minimal when $k=0$, and it is a Demoulin surface only when $k=k_{1}=k_{2}=0$. We assume that $k=0$. Then the function $\exp (\mu x+v y)$ is a solution if $\mu^{2}=v+k_{1}$ and $v^{2}=\mu+k_{2}$. According to the multiplicity of solutions $(\mu, v)$, the surface has different expressions: $Z=X^{\ell} Y^{m}$ when all solutions are distinct, $Z=n \log X-1 / n \log Y$ when two solutions coincide and $Z=\exp \left(Y-X^{2}\right)$ when three solutions coincide. Here $(X, Y, Z)$ denote the affine coordinates and $\ell, m$ and $n$ are constants.

In the case $k=0$, the system can be written as

$$
z_{x x}=z_{y}+t^{2} z, \quad z_{y y}=z_{x}+s^{2} z
$$

The invariants are $L=-2 t^{2}, M=-2 s^{2}, \sigma^{2}=s^{2}, \tau^{2}=t^{2}, \lambda=\sigma, \lambda^{\prime}=\tau$ and $\mu=\mu^{\prime}=1 / 2$ in the notation in Section 11.6. In the following $\sigma= \pm s$ and $\tau= \pm t$. The Demoulin transform is

$$
w=z_{x y}+\sigma z_{x}+\tau z_{y}+(\sigma \tau-1 / 2) z
$$

and the normalized frame is

$$
e={ }^{\mathrm{t}}\left(e_{0}, e_{1}, e_{2}, e_{3}\right)={ }^{\mathrm{t}}\left(z, z_{x}+\tau z, z_{y}+\sigma z, w\right) .
$$


Then $e$ satisfies the Pfaff equation:

$$
d e=\left(\begin{array}{cccc}
-\tau d x-\sigma d y & d x & d y & 0 \\
-\sigma d x+\frac{1}{2} d y & \tau d x-\sigma d y & d x & d y \\
\frac{1}{2} d x-\tau d y & d y & -\tau d x+\sigma d y & d x \\
0 & \frac{1}{2} d x+\tau d y & \sigma d x+\frac{1}{2} d y & \tau d x+\sigma d y
\end{array}\right) e
$$

With the notation above, it is easy to compute the system satisfied by $w$, which turns out to be the same system satisfied by $z$; namely, Demoulin transforms do not yield any new surfaces and, rather, the transforms give line congruences among the coincidence surfaces. The second Demoulin transform $u$ is given by

$$
u=w_{x y}+\sigma^{\prime} w_{x}+\tau^{\prime} w_{y}+\left(\sigma^{\prime} \tau^{\prime}-1 / 2\right) w,
$$

where $\sigma^{\prime}= \pm s$ and $\tau^{\prime}= \pm t$. A computation shows that

$$
\begin{aligned}
u= & \left(\sigma+\sigma^{\prime}\right)\left(\tau+\tau^{\prime}\right) z_{x y}+\left\{\sigma\left(\sigma+\sigma^{\prime}\right)\left(\tau+\tau^{\prime}\right)+\tau\left(\tau+\tau^{\prime}\right)+\left(\sigma+\sigma^{\prime}\right) / 2\right\} z_{x} \\
& +\left\{\tau\left(\sigma+\sigma^{\prime}\right)\left(\tau+\tau^{\prime}\right)+\sigma\left(\sigma+\sigma^{\prime}\right)+\left(\tau+\tau^{\prime}\right) / 2\right\} z_{y} \\
& +\left\{\sigma \tau\left(\sigma+\sigma^{\prime}\right)\left(\tau+\tau^{\prime}\right)+\sigma^{2}\left(\sigma+\sigma^{\prime}\right)+\tau^{2}\left(\tau+\tau^{\prime}\right)-\left(\sigma \tau+\sigma^{\prime} \tau^{\prime}\right) / 2+1 / 4\right\} z
\end{aligned}
$$

and we get the following list:

$$
\begin{array}{llll}
T 1 & z_{x y}+s z_{x}+t z_{y}+(s t-1 / 2) z & T 2 & z_{x y}-s z_{x}+t z_{y}+(-s t-1 / 2) z \\
T 3 & z_{x y}+s z_{x}-t z_{y}+(-s t-1 / 2) z & T 4 & z_{x y}-s z_{x}-t z_{y}+(s t-1 / 2) z \\
T 12 & 2 t^{2} z_{x}+t z_{y}+\left(2 t^{3}+1 / 4\right) z & T 13 & s z_{x}+2 s^{2} z_{y}+\left(2 s^{3}+1 / 4\right) z \\
T 24 & -s z_{x}+2 s^{2} z_{y}+\left(-2 s^{3}+1 / 4\right) z & T 34 & 2 t^{2} z_{x}-t z_{y}+\left(-2 t^{3}+1 / 4\right) z \\
T 14 & S(\sigma=t, \tau=s) & T 24 & S(\sigma=t, \tau=-s) \\
T 34 & S(\sigma=-t, \tau=s) & T 44 & S(\sigma=-t, \tau=-s),
\end{array}
$$

where $S(\sigma, \tau)$ denotes the surface given by the vector

$$
\begin{aligned}
4 \sigma \tau z_{x y} & +\left(4 \sigma^{2} \tau+2 \tau^{2}+\sigma\right) z_{x}+\left(4 \sigma \tau^{2}+2 \sigma^{2}+\tau\right) z_{y} \\
& +\left(4 \sigma^{2} \tau^{2}+2 \sigma^{3}+2 \tau^{3}-\sigma \tau+1 / 4\right) z .
\end{aligned}
$$

Remark 12.8. The contents for Sections 12.1 and 12.2 are based on [May32] and [Su36b, Su57] with modification relying only on the moving frame. We refer the reader to [Mar79] for Section 12.3. 


\section{Transformations of projectively minimal surfaces}

In Section 5.6, we showed the formula for Tzitzeica transformation of affine spheres. This section gives its generalization, a formula that transforms a given projectively minimal surface to a new projectively minimal surface. The formula is originally due to Marcus [Mar80].

\subsection{W-congruences of projectively minimal surfaces}

In Section 10, we exhibited how to construct $W$-congruences, where one of the focal surfaces is given by the system

$$
z_{x x}=\theta_{x} z_{x}+b z_{y}+p z, \quad z_{y y}=c z_{x}+\theta_{y} z_{y}+q z,
$$

and the other focal surface is defined by

$$
w=\mu z+2\left(A z_{x}+B z_{y}\right) .
$$

In this section, we restrict our attention to projectively minimal surfaces.

We recall that we have assumed (10.5):

$$
A_{y}+c B=0, \quad B_{x}+b A=0
$$

so that the congruence is a $W$-congruence, and we defined $\mu$ by

$$
\mu=-A_{x}-A \theta_{x}-B_{y}-B \theta_{y} .
$$

We have also defined $\mathcal{L}$ and $\mathcal{M}$ in (10.11):

$$
\mathcal{L}=A A_{x x}-\frac{1}{2} A_{x}^{2}+A^{2} L, \quad \mathcal{M}=B B_{y y}-\frac{1}{2} B_{y}^{2}+B^{2} M,
$$

and saw that

$$
N=2(\mathcal{M}-\mathcal{L})
$$

We assume here that $N \neq 0$.

Lemma 13.1. Assume the surface is projectively minimal. Then, $\mathcal{L}, \mathcal{M}$ and $N$ satisfy the same equation

$$
X_{x y}+\frac{c B}{A} X_{x}+\frac{b A}{B} X_{y}=0 .
$$

Proof. Making use of the identity (13.1) and one of the integrability conditions $L_{y}=-2 b c_{x}-c b_{x}$, we see that

$$
\mathcal{L}_{x y}+\frac{c B}{A} \mathcal{L}_{x}+\frac{b A}{B} \mathcal{L}_{y}=-A B\left(c_{x x x}+c L_{x}+2 c_{x} L\right) .
$$

The condition of projective minimality implies that the right-hand side vanishes and we get the result for $\mathcal{L}$. The case for $\mathcal{M}$ is similarly done. 
The invariants for the surface $w$ were given in (10.9). By the expressions of these invariants, we can compute $L$ and $M$ for $w$, which we denote by $\bar{L}$ and $\bar{M}$ :

$$
\begin{aligned}
& \bar{L}=L+\frac{N_{x x}}{N}-\frac{3}{2}\left(\frac{N_{x}}{N}\right)^{2}+\frac{A_{x}}{A} \frac{N_{x}}{N}, \\
& \bar{M}=M+\frac{N_{y y}}{N}-\frac{3}{2}\left(\frac{N_{y}}{N}\right)^{2}+\frac{B_{y}}{B} \frac{N_{y}}{N} .
\end{aligned}
$$

We also recall $\Delta_{1}$ and $\Delta_{2}$ given in (11.5):

$$
\Delta_{1}=b_{y}^{2}-2 b b_{y}-2 b^{2} M, \quad \Delta_{2}=c_{x}^{2}-2 c c_{x}-2 c^{2} L .
$$

Then we have the following lemma on such invariants for $w$ by direct computation.

LEMMA 13.2. Assume that the surface $z$ is projectively minimal. Then

$$
\bar{\Delta}_{1}=\Delta_{1}+\frac{4 N_{x}}{A^{2} N^{2}}\left(\mathcal{M} \mathcal{L}_{x}-\mathcal{L} \mathcal{M}_{x}\right), \quad \bar{\Delta}_{2}=\Delta_{2}+\frac{4 N_{y}}{B^{2} N^{2}}\left(\mathcal{M} \mathcal{L}_{y}-\mathcal{L} \mathcal{M}_{y}\right) .
$$

Provided that $b c \neq 0$, the conditions $\Delta_{1 y}=0$ and $\Delta_{2 x}=0$ are equivalent, and both imply the surface is projectively minimal; see (11.6). Hence, we have the following proposition.

Proposition 13.3. Assume that the surface $z$ is projectively minimal and that

$$
\mathcal{M}=k \mathcal{L}
$$

for a constant $k \neq 1$. Then the surface $w$ is also projectively minimal.

Proof. From $\mathcal{M}=k \mathcal{L}$, we see that $\bar{\Delta}_{1}=\Delta_{1}$. Hence $\bar{\Delta}_{1}$ is independent of $y$, which implies the result. $k \neq 1$ is necessary for $N \neq 0$.

Moreover, as $\Delta_{1}=\Delta_{2}=0$ means that the surface $z$ is a Demoulin surface, and as either one of the conditions $\Delta_{1}=0$ and $\Delta_{2}=0$ implies that the surface is a Godeaux-Rozet surface, we have the following proposition.

PROPOSITION 13.4. Assume that the surface $z$ is projectively minimal and that

$$
\mathcal{M}=k \mathcal{L}
$$

for some constant $k \neq 1$. If $z$ is a Godeaux-Rozet surface, then so is $w$. If $z$ is a Demoulin surface, then so is $w$. 


\subsection{Transformations of projectively minimal surfaces}

We now apply the propositions above by finding $A$ and $B$ satisfying (13.1) and (13.2). Let us consider the surface given by

$$
z_{x x}=b z_{y}+\left(\pi-\frac{1}{2} b_{y}\right) z, \quad z_{y y}=c z_{x}+\left(\rho-\frac{1}{2} c_{x}\right) z
$$

so that $L=-2 \pi$ and $M=-2 \rho$ take simple forms. We assume that $z$ is projectively minimal:

$$
b M_{y}+2 M b_{y}+b_{y y y}=0, \quad c L_{x}+2 L c_{x}+c_{x x x}=0 .
$$

For a parameter $\alpha(\neq 1)$, we define a new system

$$
\bar{z}_{x x}=\alpha b \bar{z}_{y}+\left(\pi-\frac{\alpha}{2} b_{y}\right) \bar{z}, \quad \bar{z}_{y y}=\frac{1}{\alpha} c \bar{z}_{x}+\left(\rho-\frac{1}{2 \alpha} c_{x}\right) \bar{z} .
$$

As $L=-2 \pi$ and $M=-2 \rho$ also for this system, $L$ and $M$ are unchanged and the product $b c$ is also unchanged. Hence, the system for $\bar{z}$ is integrable.

We now let $\varphi$ and $\psi$ be two independent solutions of (13.4) and define

$$
A=-\alpha\left(\psi \varphi_{y}-\varphi \psi_{y}\right), \quad B=\psi \varphi_{x}-\varphi \psi_{x}
$$

It is easy to see that the condition (13.2) is satisfied; in fact

$$
\begin{aligned}
A_{y} & =-\alpha\left(\psi \varphi_{y y}-\varphi \psi_{y y}\right) \\
& =-\alpha\left(\psi\left(\frac{1}{\alpha} c \varphi_{x}+\left(\rho-\frac{1}{2} c_{x}\right) \varphi\right)-\varphi\left(\frac{1}{\alpha} c \psi_{x}+\left(\rho-\frac{1}{2} c_{x}\right) \psi\right)\right) \\
& =-c B .
\end{aligned}
$$

Furthermore, by computation, we see that

$$
\begin{aligned}
\frac{2}{\alpha^{2}} \mathcal{L}= & 2 b c\left(\psi \varphi_{y}-\varphi \psi_{y}\right)\left(\psi \varphi_{x}-\varphi \psi_{x}\right)-\left(\varphi_{x} \psi_{y}-\psi_{x} \varphi_{y}\right)^{2}-\left(\varphi \psi_{x y}-\psi \varphi_{x y}\right)^{2} \\
& +\left(-4 \psi \varphi_{x} \varphi_{y}+2 \varphi\left(\varphi_{x} \psi_{y}+\psi_{x} \varphi_{y}\right)\right) \psi_{x y} \\
& +\left(-4 \varphi \psi_{x} \psi_{y}+2 \psi\left(\varphi_{x} \psi_{y}+\psi_{x} \varphi_{y}\right)\right) \varphi_{x y}
\end{aligned}
$$

The computation of $2 \mathcal{M}$ yields the same right-hand side. Namely, we have $\mathcal{L}=\alpha^{2} \mathcal{M}$.

THEOREM 13.5. Assume that the surface (13.3) is projectively minimal. For any constant $\alpha(\neq \pm 1)$ and independent solutions $\varphi$ and $\psi$ of the system (13.4), define $A$ and $B$ by (13.5), and define $\mu$ by

$$
\mu=(\alpha+1)\left(\psi_{x} \varphi_{y}-\varphi_{x} \psi_{y}\right)+(\alpha-1)\left(\psi \varphi_{x y}-\varphi \psi_{x y}\right) .
$$


Then the surface

$$
w=\mu z+2\left(A z_{x}+B z_{y}\right)
$$

defines a projectively minimal surface. If $z$ is a Demoulin (respectively GodeauxRozet) surface, then so is $w$.

Let $\varphi_{1}, \ldots, \varphi_{4}$ be independent solutions of the system (13.4). Then $\varphi$ and $\psi$ are linear combinations of these solutions, say $\varphi=\sum_{i} k^{i} \varphi_{i}$ and $\psi=\sum_{i} m^{i} \varphi_{i}$. For $i<j$, we define the coefficients

$$
\begin{gathered}
A_{i j}=-\alpha\left(\varphi_{j} \varphi_{i y}-\varphi_{i} \varphi_{j y}\right), \quad B_{i j}=\varphi_{j} \varphi_{i x}-\varphi_{i} \varphi_{j x}, \\
\mu_{i j}=(\alpha+1)\left(\varphi_{j x} \varphi_{i y}-\varphi_{i x} \varphi_{j y}\right)+(\alpha-1)\left(\varphi_{j} \varphi_{i x y}-\varphi_{i} \varphi_{j x y}\right),
\end{gathered}
$$

and the surface

$$
w_{i j}=\mu_{i j} z+2\left(A_{i j} z_{x}+B_{i j} z_{y}\right) .
$$

Then, the surface $w$ associated with the pair $\varphi$ and $\psi$ is a combination of $w_{i j}$ :

$$
w=\sum_{i<j} c^{i j} w_{i j}
$$

where $c^{i j}=k^{i} m_{j}-k^{j} m^{i}$. The coefficients $c^{i j}$ can be regarded as the wedge product of the vectors $\left(k^{i}\right)$ and $\left(m^{j}\right)$ and they satisfy the Plücker relation $c^{12} c^{34}-c^{13} c^{24}+$ $c^{14} c^{23}=0$. We remark that the system satisfied by the surface $w$ can be computed by using the formulas (10.8) and (10.9).

We next modify the transformation formula in Theorem 13.5 to show that the Tzitzeica transformation is derived from the above transformation. We start with the projectively minimal surface $z$ given by the system of a general form

$$
z_{x x}=\theta_{x} z_{x}+b z_{y}+p z, \quad z_{y y}=c z_{x}+\theta_{y} z_{y}+q z
$$

and then we introduce another system by

$$
\bar{z}_{x x}=\bar{\theta}_{x} \bar{z}_{x}+\alpha b \bar{z}_{y}+\overline{p z}, \quad \bar{z}_{y y}=\frac{c}{\alpha} \bar{z}_{x}+\bar{\theta}_{y} \bar{z}_{y}+\overline{q z},
$$

so that $\bar{L}=L$ and $\bar{M}=M$. As both systems can be normalized to the forms in (13.3) and (13.4) by multiplying $z$ and $\bar{z}$ by some factors, existence of such a system is trivial; however, finding explicit representations of $\bar{\theta}, \bar{p}$, and $\bar{q}$ is not simple. Notwithstanding this ambiguity, we define $A$ and $B$ by

$$
A=-\alpha \exp (-\bar{\theta})\left(\psi \varphi_{y}-\varphi \psi_{y}\right), \quad B=\exp (-\bar{\theta})\left(\psi \varphi_{x}-\varphi \psi_{x}\right)
$$


Furthermore, we can modify the systems above so that $\bar{p}=0$ and $\bar{q}=0$ :

$$
z_{x x}=\theta_{x} z_{x}+b z_{y}, \quad z_{y y}=c z_{x}+\theta_{y} z_{y},
$$

and

$$
\bar{z}_{x x}=\bar{\theta}_{x} \bar{z}_{x}+\alpha b \bar{z}_{y}, \quad \bar{z}_{y y}=\frac{c}{\alpha} \bar{z}_{x}+\bar{\theta}_{y} \bar{z}_{y},
$$

so that $\psi=1$ is a trivial solution. Then, we get

$$
A=-\alpha \exp (-\bar{\theta}) \varphi_{y}, \quad B=\exp (-\bar{\theta}) \varphi_{x} .
$$

We assume further that $\bar{\theta}=\theta$. Then, $b \theta_{y}+b_{y}=0$ and $c \theta_{x}+c_{x}=0$ are necessary to conclude that $\bar{L}=L$ and $\bar{M}=M$. As $(\log (b / c))_{x y}=0$ in this case, we can assume that $c=b$. Now the situation is reduced to the case where the original system has the form

$$
z_{x x}=-\frac{b_{x}}{b} z_{x}+b z_{y}, \quad z_{y y}=b z_{x}-\frac{b_{y}}{b} z_{y} .
$$

The integrability condition is

$$
(\log b)_{x y}=b^{2}+\frac{k}{b}
$$

where $k$ is any constant. If we set $b=1 / h$, then these equations are written as

$$
z_{x x}=\frac{h_{x}}{h} z_{x}+\frac{1}{h} z_{y}, \quad z_{y y}=\frac{1}{h} z_{x}+\frac{h_{y}}{h} z_{y} .
$$

The integrability condition is

$$
(\log h)_{x y}=-k h-\frac{1}{h^{2}} .
$$

This is the case we have considered in Section 5.6 for Tzitzeica transformation of affine spheres. In fact, setting $k=-1$, we assume that $z$ also satisfies

$$
z_{x y}=h z,
$$

so that $z$ defines an affine sphere. Let $\varphi$ be a non-trivial solution of

$$
\bar{z}_{x x}=\frac{h_{x}}{h} \bar{z}_{x}+\frac{\alpha}{h} \bar{z}_{y}, \quad \bar{z}_{y y}=\frac{1}{\alpha h} \bar{z}_{x}+\frac{h_{y}}{h} \bar{z}_{y},
$$

and assume that it also satisfies

$$
\varphi_{x y}=h \varphi .
$$

Then we get the transformed surface

$$
w=\frac{1}{2}(\alpha-1) \varphi z-\frac{\alpha \varphi_{y}}{h} z_{x}+\frac{\varphi_{x}}{h} z_{y},
$$


which is a new affine sphere. This gives nothing but the Tzitzeica transformation stated in Theorem 5.10.

Remark 13.6. In [FS99], Ferapontov and Schief gave the transformation formula of Theorem 5.10 relative to Demoulin surfaces by appealing to the system of nonlinear equations (2.27) and using the Moutard transformation. Their formulation was generalized in [RS02] to projectively minimal surfaces by Rogers and Schief, resulting in the same formula.

Acknowledgements. This article is based on the author's lectures and talks in several universities and he would like to express his sincere thanks to the colleagues who have afforded him the opportunity, and especially to Masaaki Yoshida, Wayne Rossman and Masayuki Noro for their helpful suggestions and remarks. This article was completed during the author's stay at MPI für Mathematik in Bonn in 2005; he would like to express his gratitude to the institute. Financial support is due in part to JSPS-Kakenhi (Grant-in-Aid for Scientific Research) C17540076.

\section{Appendix A. Line congruences derived from Appell's system $\left(F_{2}\right)$}

Appell's system is defined in Section 4.7:

$$
D_{1} z=0 \text { and } D_{2} z=0 \text {, }
$$

where

$$
\begin{gathered}
D_{1}=x(1-x) \partial_{x x}-x y \partial_{x y}+\{\gamma-(\alpha+\beta+1) x\} \partial_{x}-\beta y \partial_{y}-\alpha \beta, \\
D_{2}=y(1-y) \partial_{y y}-x y \partial_{x y}+\left\{\gamma^{\prime}-\left(\alpha+\beta^{\prime}+1\right) y\right\} \partial_{y}-\beta^{\prime} x \partial_{x}-\alpha \beta^{\prime} .
\end{gathered}
$$

Appell's function $F_{2}=F_{2}\left(\alpha, \beta, \beta^{\prime}, \gamma, \gamma^{\prime} ; x, y\right)$ is also given in Section 4.7. When certain number of parameters are increased or decreased by 1 , the set of parameters is said to be contiguous to the original set of parameters and any function $F_{2}$ with (a set of) contiguous parameters, say $F_{2}\left(\alpha \pm 1, \beta, \beta^{\prime}, \gamma \cdot \gamma^{\prime} ; x, y\right)$, $F_{2}(\alpha \pm 1, \beta \pm 1, \ldots ; x, y), \ldots$, is called a contiguous function of $F_{2}$. As the dimension of the solution space of the system is four, contiguous functions are written by using $F_{2},\left(F_{2}\right)_{x},\left(F_{2}\right)_{y}$ and $\left(F_{2}\right)_{x y}$, and formulas representing such relations are called contiguity relations. However, some contiguous functions are written by using only the first three, not including the second-order derivative. For example, the operator $H_{0}$ increases $\beta$ by +1 and the operator $B_{0}$ decreases $\beta$ by -1 and both give contiguous functions; $H_{n}$ and $B_{n}$ are defined in Section 4.7. Geometrically speaking, in such 
a case, any point of the surface defined by the system of contiguous parameters (to be called a contiguous surface) is lying on the tangent plane at the point of the same coordinates of the original surface. We refer the reader to [Sa91] for the list of the basic contiguous relations of Appell's system corresponding to Appell's function usually denoted by $F_{3}$; note that the system is equivalent to $\left(F_{2}\right)$. From the list, we can read that the pair of $H_{0}$ and $B_{0}$ is essentially unique in the sense that the contiguous surface is lying on the tangent space of the original surface as well as the original surface is lying on the tangent space of the contiguous surface. This means that these two surfaces are focal surfaces of the line congruence joining them. In the following, we describe explicitly this congruence in terms of Laplace transformation.

As the invariant quadratic form is conformal to $(y /(1-x)) d x^{2}+2 d x d y+$ $(x /(1-y)) d y^{2}$, one choice of conjugate directional fields is given by $(x-1) \partial_{x}$ and $(x-1) \partial_{x}+y \partial_{y}$. We adopt here the notation

$$
X=(x-1) \partial_{x}, \quad Y=(x-1) \partial_{x}+y \partial_{y} .
$$

They are commutative: $[X, Y]=0$.

In terms of $X$ and $Y$, the operators $D_{1}$ and $D_{2}$ are written as follows:

$$
\begin{aligned}
& D_{1}=-\frac{1}{x-1}(x X Y+((\beta-\gamma)+\alpha x) X+\beta(x-1) Y+\alpha \beta(x-1)), \\
& D_{2}=-\frac{y-1}{y} Y Y+\left(\frac{x}{x-1}-\frac{y-1}{y}\right) X X+A Y+B X+C,
\end{aligned}
$$

where

$$
\begin{gathered}
A=-2 \beta \frac{(x-1)(y-1)}{x y}+\beta-\beta^{\prime}-\alpha+\frac{\gamma^{\prime}-1}{y}, \\
B=\frac{2(\beta-\gamma)(1-y)}{x y}+\frac{2 \alpha+1-\gamma^{\prime}}{y}+\frac{\alpha+\beta-\beta^{\prime}-\gamma}{x-1}, \\
C=-\frac{2 \alpha \beta(x-1)(y-1)}{x y}+\alpha\left(\beta-\beta^{\prime}\right) .
\end{gathered}
$$

The first equation is

$$
X Y z+\left(\frac{\beta-\gamma}{x}+\alpha\right) X z+\beta \frac{x-1}{x} Y z+\alpha \beta \frac{x-1}{x} z=0 .
$$

Hence, the Laplace transforms are

$$
z^{1}=Y z+\left(\frac{\beta-\gamma}{x}+\alpha\right) z, \quad z^{-1}=X z+\beta \frac{x-1}{x} z,
$$


by definition. Note that $z^{1}$ is equal to $H_{0} z$ and $z^{-1}$ to $B_{0} z$ up to scalar multiple. The Laplace invariants are

$$
h=(\beta-1)(\beta-\gamma) \frac{x-1}{x^{2}}, \quad k=\beta(\beta-\gamma+1) \frac{x-1}{x^{2}}-\alpha \beta \frac{x-1}{x} .
$$

The Laplace equation for $z^{1}$ is, by computation,

$$
X Y z^{1}+\left(\alpha+1+\frac{\beta-\gamma-2}{x}\right) X z^{1}+\beta \frac{x-1}{x} Y z^{1}+\left(\beta(\alpha+1) \frac{x-1}{x}-\gamma \frac{x-1}{x^{2}}\right) z^{1}=0 .
$$

We next define a projective frame to show how to compute the invariants of the line congruence $\left\{z, z^{-1}\right\}$ by

$$
\begin{gathered}
e_{1}=x^{\beta} z \\
e_{2}=x^{\gamma-\beta-1}(x-1)^{\alpha+\beta-\gamma}(x X z+\beta(x-1) z), \\
e_{3}=x^{\beta}\left(Y z+\frac{\beta(x-1)}{x} z\right), \\
e_{4}=X e_{2},
\end{gathered}
$$

and introduce coordinates $(u, v)$ by requiring $X=\partial_{u}$ and $Y=\partial_{v}$. Then for any function $f$ we have $d f=(X f) d u+(Y f) d v$. By using the expressions (A.2), we can prove the following:

$$
\begin{gathered}
\omega^{1}=\omega_{1}^{3}=d v, \quad \omega^{2}=\omega_{2}^{4}=d u, \\
\omega_{1}^{2}=x^{2 \beta-\gamma}(x-1)^{\gamma-\alpha \beta} d u, \\
\omega_{2}^{1}=\beta(\beta-\gamma+1) x^{\gamma-2 \beta-2}(x-1)^{\alpha+\beta-\gamma+1} d v, \\
\omega_{3}^{4}=\frac{x+y-1}{y-1} x^{2 \beta-\gamma}(x-1)^{\gamma-\alpha-\beta-1} d v, \\
\omega_{4}^{3}=\beta(\beta-\gamma+2) \frac{y-1}{x+y-1} x^{\gamma-2 \beta-2}(x-1)^{\alpha+\beta-\gamma+2} d u .
\end{gathered}
$$

Therefore, by the definition of the invariant $W$ in Section 4, we see that

$$
W=h_{12} h_{21}-h_{31} h_{42}=-\beta \frac{x-1}{x^{2}},
$$

which means the line congruence $\left\{z, z^{-1}\right\}$ is a $W$-congruence only when $\beta=0$. 


\section{Appendix B. Line congruences in $\mathbf{E}^{3}$}

Let us give a brief summary of the theory of line congruences in the three-dimensional Euclidean space $\mathbf{E}^{3}$, first formulated by Kummer [Ku60], and then explain how the notion of $W$-congruence was introduced.

Each line in the congruence parametrized by $(x, y)$ is described by the pair consisting of a base point $z(x, y)$ on the line and the directional unit vector $\phi(x, y)$. The line is expressed as $z(x, y)+t \phi(x, y)$ with the line parameter $t$. On the other hand, we can regard $z(x, y)$ as a surface and $\phi(x, y)$ as a spherical surface. The surface $z$ is called a reference surface. If we choose another reference surface $z^{\prime}(x, y)$, we have a relation such as $z^{\prime}(x, y)=z(x, y)+\ell \phi(x, y)$. The fundamental invariants related to this pair are the metric form of the spherical surface

$$
\mathrm{I}=\langle d \phi, d \phi\rangle=E d x^{2}+2 F d x d y+G d y^{2},
$$

and the 2-form

$$
\mathbb{I}=-\langle d z, d e\rangle=p d x^{2}+\left(q+q^{\prime}\right) d x d y+r d y^{2},
$$

where

$$
\begin{gathered}
E=\left\langle\phi_{x}, \phi_{x}\right\rangle, \quad F=\left\langle\phi_{x}, \phi_{y}\right\rangle, \quad G=\left\langle\phi_{y}, \phi_{y}\right\rangle, \\
p=\left\langle\phi_{x}, z_{x}\right\rangle, \quad q=\left\langle\phi_{x}, z_{y}\right\rangle, \quad q^{\prime}=\left\langle\phi_{y}, z_{x}\right\rangle, \quad r=\left\langle\phi_{y}, z_{y}\right\rangle .
\end{gathered}
$$

The form II for the reference surface $z^{\prime}$, denoted by $\mathbb{I}^{\prime}$, is

$$
\mathbb{I}^{\prime}=\mathbb{I}+\ell \mathbf{I} .
$$

If we write $\mathrm{I}$ as $\sum g_{i j} d x^{i} d x^{j}$, where $x^{1}=x$ and $x^{2}=y$, then the Gauss equation for the map $\phi$ is

$$
\phi_{i j}=\sum_{k} \Gamma_{i j}^{k} \phi_{k}-g_{i j} z
$$

where $\Gamma_{i j}^{k}$ are the Christoffel symbols relative to $\left\{g_{i j}\right\}$.

The line congruence is called a normal congruence if there exists a surface $z^{\prime}$ such that $\phi$ coincides with the unit normal of the surface $z^{\prime}$. The condition is $\left\langle d z^{\prime}, \phi\right\rangle=0$, which implies that $\langle d z, \phi\rangle+d t=0$. Then, by exterior differentiation, we must have $\left\langle z_{x}, \phi_{y}\right\rangle=\left\langle z_{y}, \phi_{x}\right\rangle$, namely, $q=q^{\prime}$. Conversely, if $q=q^{\prime}$, we can find such a $z^{\prime}$. 
The focal points on each line are $Z(x, y)=z(x, y)+t \phi(x, y)$ for which $d Z$ is parallel to $\phi$. This implies that II $+t \mathrm{I}=0$. In particular, the value $t$ is determined by the quadratic equation

$$
\left|\begin{array}{cc}
p+t E & q+t F \\
q^{\prime}+t F & r+t G
\end{array}\right|=0
$$

For each $t$, the direction $d x: d y$ determines the degenerate ruled surface, in which lies a focal point.

The fundamental equations of the congruence $\{z, \phi\}$ are the Gauss equation (B.3) and the system

$$
\begin{gathered}
z_{x}=a \phi_{x}+b \phi_{y}+m \phi, \\
z_{y}=c \phi_{x}+d \phi_{y}+n \phi .
\end{gathered}
$$

The coefficients are determined by the formula

$$
\left(\begin{array}{ll}
a & b \\
c & d
\end{array}\right)=\left(\begin{array}{ll}
p & q^{\prime} \\
q & r
\end{array}\right)\left(\begin{array}{ll}
E & F \\
F & G
\end{array}\right), \quad m=\left\langle\phi, z_{x}\right\rangle, \quad n=\left\langle\phi, z_{y}\right\rangle .
$$

The integrability condition of the last system is computed by developing the equation $\left(z_{x}\right)_{y}=\left(z_{y}\right)_{x}$ into a linear combination of $\phi, \phi_{x}$ and $\phi_{y}$, by using (B.3). The resulting equations are

$$
\begin{gathered}
a_{y}+a \Gamma_{12}^{1}+b \Gamma_{22}^{1}-c_{x}-c \Gamma_{11}^{1}-d \Gamma_{12}^{1}-n=0, \\
b_{y}+a \Gamma_{12}^{2}+b \Gamma_{22}^{2}-d_{x}-c \Gamma_{11}^{2}-d \Gamma_{12}^{2}+m=0, \\
m_{y}-n_{x}-a F-b G+c E+d F=0 .
\end{gathered}
$$

By making use of the formula expressing the Christoffel symbol $\Gamma_{i j}^{k}$ in terms of $E, F$ and $G$, we can see that the system above is equivalent to the system

$$
\begin{gathered}
p_{y}-q_{x}-\Gamma_{12}^{1} p+\Gamma_{11}^{1} q-\Gamma_{12}^{2} q^{\prime}+\Gamma_{11}^{2} r+F m-E n=0, \\
q_{y}^{\prime}-r_{x}-\Gamma_{22}^{1} p+\Gamma_{12}^{1} q-\Gamma_{22}^{2} q^{\prime}+\Gamma_{12}^{2} r+G m-F n=0, \\
m_{y}-n_{x}+q-q^{\prime}=0 .
\end{gathered}
$$

We summarize the above argument as follows.

THEOREM B.1. The line congruence $\{\phi, z\}$ described above is determined by the systems (B.3) and (B.4) satisfying the integrability condition (B.5). Conversely, 
given a spherical surface $\phi$ and the coefficients $p, q, q^{\prime}, r, m$ and $n$ satisfying (B.5), any solution of (B.4) gives a reference surface $z$, and thus the line congruence.

Example B.2. Let us consider the normal congruence for a surface in the Euclidean space. The surface is $z$ and $\phi$ is its Gauss mapping. The shape operator $A$ is given by $\nabla_{X} \phi=-A X$. The directional field $d x: d y$ for determining the focal points is given by $-A X \equiv 0(\bmod X)$. The integral curves are nothing but the curvature lines.

A surface in $\mathbf{E}^{3}$ is called a Weingarten surface if there exists a functional relation between the two principal curvatures. We here recall how normal congruence is related to the $W$-congruence.

Assume that $(x, y)$ are the curvature coordinates so that the first fundamental form of the surface is $e d x^{2}+g d y^{2}$ (to avoid confusion with the first fundamental form I for the surface $\phi$, we do not use the usual notation $E$ and $G$ ) and the second fundamental form is nothing but II above with the condition $q=q^{\prime}=0$.

The principal curvatures $k_{1}$ and $k_{2}$ are given by $k_{1}=p / e, k_{2}=r / g$.

The Codazzi identity is written as

$$
\left(k_{2}\right)_{x}+\frac{1}{2}\left(k_{2}-k_{1}\right) \frac{\partial \log g}{\partial x}=0, \quad\left(k_{1}\right)_{y}+\frac{1}{2}\left(k_{1}-k_{2}\right) \frac{\partial \log e}{\partial y}=0 .
$$

We assume that $k_{1} k_{2} \neq 0$ and set $R_{i}=1 / k_{i}$, which are the principal radii. Then the coordinates have the property that

$$
z_{x}+R_{1} \phi_{x}=0, \quad z_{y}+R_{2} \phi_{y}=0 .
$$

For the surface $z$, we define two mappings by

$$
w_{i}=z+R_{i} \phi \quad i=1,2 .
$$

They define in general two surfaces called the central surfaces. (We do not treat the case where these mappings are degenerate.)

Let $w=w_{1}$ for the moment. The relation above shows that

$$
w_{x}=R_{1 x} \phi, \quad w_{y}=\left(1-R_{1} / R_{2}\right) z_{y}+R_{1 y} \phi,
$$

which imply that the unit normal to $w$ is $\phi_{1}=z_{x} / \sqrt{e}$ up to sign. Then it is easily seen, by using the Coddazi identity, that the coefficients of the second fundamental 
form of $w$, denoted by $p_{1}, q_{1}$ and $r_{1}$, are given by

$$
p_{1}=-\sqrt{e}\left(\log R_{1}\right)_{x}, \quad q_{1}=0, \quad r_{1}=-\frac{g R_{1}}{\sqrt{e} R_{2}}\left(\log R_{2}\right)_{y} .
$$

Thus, the second fundamental form of the first surface is conformal to

$$
e R_{2}^{2} R_{1 x} d x^{2}-g R_{1}^{2} R_{2 x} d y^{2} .
$$

Similarly, for the second surface we have

$$
e R_{2}^{2} R_{1 y} d x^{2}-g R_{1}^{2} R_{2 y} d y^{2} .
$$

These computations show the following theorem.

THEOREM B.3. Assume the central surfaces are non-degenerate. Then the normal congruence between them is a $W$-congruence if and only if the principal radii satisfy the relation

$$
\left|\begin{array}{ll}
R_{1 x} & R_{2 x} \\
R_{1 y} & R_{2 y}
\end{array}\right|=0 .
$$

The original surface satisfying this relation is classically called a Weingarten surface, and this is the origin of the naming of $W$-congruence.

For a general theory of normal congruence, we refer the reader to, e.g., [D2, nos 447-455] and [E09, Ch. XII].

\section{Appendix C. Plücker image of projective surfaces into $\mathbb{P}^{5}$}

We here give a proof of Theorem 4.25. From the assumption $(t, t)=h$, the connection form $\omega$ satisfies $\omega h+h^{\mathrm{t}} \omega=0$. Together with the condition $\omega_{1}^{4}=\omega_{1}^{5}=\omega_{1}^{6}=0$ and $\omega_{2}^{3}=\omega_{2}^{5}=\omega_{2}^{6}=0$ from (4.14), the form $\omega$ can be written as

$$
\omega=\left(\begin{array}{cccccc}
\omega_{1}^{1} & \omega_{1}^{2} & \omega^{2} & 0 & 0 & 0 \\
\omega_{2}^{1} & \omega_{2}^{2} & 0 & \omega^{1} & 0 & 0 \\
\omega_{3}^{1} & \omega_{3}^{2} & 0 & \sigma & \omega^{2} & 0 \\
\omega_{4}^{1} & \omega_{4}^{2} & \sigma & 0 & 0 & \omega^{1} \\
0 & \tau & \omega_{3}^{1} & -\omega_{4}^{1} & -\omega_{1}^{1} & \omega_{2}^{1} \\
\tau & 0 & -\omega_{3}^{2} & \omega_{4}^{2} & \omega_{1}^{2} & -\omega_{2}^{2}
\end{array}\right),
$$


where we set $\sigma=\omega_{3}^{4}$ and $\tau=\omega_{5}^{2}$. It satisfies the following integrability conditions:

$$
\begin{aligned}
d \omega_{1}^{1} & =\omega_{1}^{2} \wedge \omega_{2}^{1}+\omega^{2} \wedge \omega_{3}^{1}, \\
d \omega_{1}^{2} & =\omega_{1}^{1} \wedge \omega_{1}^{2}+\omega_{1}^{2} \wedge \omega_{2}^{2}+\omega^{2} \wedge \omega_{3}^{2}, \\
d \omega_{2}^{1} & =\omega_{2}^{1} \wedge \omega_{1}^{1}+\omega_{2}^{2} \wedge \omega_{2}^{1}+\omega^{1} \wedge \omega_{4}^{1}, \\
d \omega_{2}^{2} & =\omega_{2}^{1} \wedge \omega_{1}^{2}+\omega^{1} \wedge \omega_{4}^{2}, \\
d \omega^{1} & =\omega_{2}^{2} \wedge \omega^{1}, \\
d \omega^{2} & =\omega_{1}^{1} \wedge \omega^{2}, \\
d \omega_{3}^{1} & =\omega_{3}^{1} \wedge \omega_{1}^{1}+\omega_{3}^{2} \wedge \omega_{2}^{1}+\sigma \wedge \omega_{4}^{1}, \\
d \omega_{3}^{2} & =\omega_{3}^{1} \wedge \omega_{1}^{2}+\omega_{3}^{2} \wedge \omega_{2}^{2}+\sigma \wedge \omega_{4}^{2}+\omega^{2} \wedge \tau, \\
d \omega_{4}^{1} & =\omega_{4}^{1} \wedge \omega_{1}^{1}+\omega_{4}^{2} \wedge \omega_{2}^{1}+\sigma \wedge \omega_{3}^{1}+\omega^{1} \wedge \tau, \\
d \omega_{4}^{2} & =\omega_{4}^{1} \wedge \omega_{1}^{2}+\omega_{4}^{2} \wedge \omega_{2}^{2}+\sigma \wedge \omega_{3}^{2}, \\
d \sigma & =\omega_{3}^{2} \wedge \omega^{1}-\omega^{2} \wedge \omega_{4}^{1}, \\
d \tau & =\tau \wedge \omega_{1}^{1}+\tau \wedge \omega_{2}^{2}-\omega_{4}^{1} \wedge \omega_{4}^{2}+\omega_{3}^{1} \wedge \omega_{3}^{2}, \\
0 & =\omega_{1}^{2} \wedge \omega^{1}-\sigma \wedge \omega^{2}, \\
0 & =\omega_{2}^{1} \wedge \omega^{2}-\sigma \wedge \omega^{1} .
\end{aligned}
$$

To check the action of the transformation $g$, we divide $\omega$ and $g$ into $(3 \times 3)$-matrix components:

$$
\omega=\left(\begin{array}{ccc}
\Omega_{1}^{1} & \Omega_{1}^{2} & 0 \\
\Omega_{2}^{1} & \Omega_{2}^{2} & \Omega_{2}^{3} \\
\Omega_{3}^{1} & \Omega_{3}^{2} & \Omega_{3}^{4}
\end{array}\right), \quad g=\left(\begin{array}{ccc}
G_{11} & 0 & 0 \\
G_{21} & G_{22} & 0 \\
G_{31} & G_{32} & G_{33}
\end{array}\right)
$$

Since $g h^{\mathrm{t}} g=h$, we have

$$
\begin{gathered}
G_{33}=J\left({ }^{\mathrm{t}} G_{11}\right)^{-1} J, \quad G_{22} J{ }^{\mathrm{t}} G_{22}=J, \quad G_{22} J{ }^{\mathrm{t}} G_{32}=G_{21} J{ }^{\mathrm{t}} G_{33}, \\
G_{32} J{ }^{\mathrm{t}} G_{32}=G_{33} J{ }^{\mathrm{t}} G_{31}+G_{31} J{ }^{\mathrm{t}} G_{33} .
\end{gathered}
$$

By a transformation $g, \omega$ changes to $\tilde{\omega}=d g \cdot g^{-1}+g \omega g^{-1}$ that is written as

$$
\tilde{\omega}=\left(\tilde{\omega}_{i}^{j}\right)=\left(\begin{array}{ccc}
\tilde{\Omega}_{1}^{1} & \tilde{\Omega}_{1}^{2} & 0 \\
\tilde{\Omega}_{2}^{1} & \tilde{\Omega}_{2}^{2} & \tilde{\Omega}_{2}^{3} \\
\tilde{\Omega}_{3}^{1} & \tilde{\Omega}_{3}^{2} & \tilde{\Omega}_{3}^{4}
\end{array}\right),
$$


where

$$
\begin{aligned}
\tilde{\Omega}_{1}^{1}= & d G_{11} G_{11}^{-1}+G_{11}\left(\Omega_{1}^{1}-\Omega_{1}^{2} G_{22}^{-1} G_{21}\right) G_{11}^{-1}, \\
\tilde{\Omega}_{1}^{2}= & G_{11} \Omega_{1}^{2} G_{22}^{-1}, \\
\tilde{\Omega}_{2}^{2}= & d G_{22} G_{22}^{-1}+G_{22}\left(G_{22}^{-1} G_{21} \Omega_{1}^{2}+\Omega_{2}^{2}-\Omega_{2}^{3} G_{33}^{-1} G_{32}\right) G_{22}^{-1}, \\
\tilde{\Omega}_{2}^{3}= & G_{22} \Omega_{2}^{3} G_{33}^{-1}, \\
\tilde{\Omega}_{2}^{1}= & d G_{21}-\left(d G_{22}+G_{21} \Omega_{1}^{2}+G_{22} \Omega_{2}^{2}\right) G_{22}^{-1} G_{21} G_{11}^{-1} \\
& +\left(G_{21} \Omega_{1}^{1}+G_{22} \Omega_{2}^{1}\right) G_{11}^{-1}+G_{22} \Omega_{23} G_{33}^{-1}\left(-G_{31}+G_{32} G_{22}^{-1} G_{21}\right) G_{11}^{-1}, \\
\tilde{\Omega}_{3}^{1}= & d G_{31} G_{11}^{-1}-d G_{32} G_{22}^{-1} G_{21} G_{11}^{-1} \\
& +\left(d G_{33}+G_{32} \Omega_{2}^{3}+G_{33} \Omega_{33}\right) G_{33}^{-1}\left(-G_{31}+G_{32} G_{22}^{-1} G_{21}\right) G_{11}^{-1} \\
& +\left(G_{31} \Omega_{1}^{1}+G_{32} \Omega_{2}^{1}+G_{33} \Omega_{3}^{1}\right) G_{11}^{-1} \\
& -\left(G_{31} \Omega_{1}^{2}+G_{32} \Omega_{22}+G_{33} \Omega_{3}^{2}\right) G_{22}^{-1} G_{21} G_{11}^{-1} .
\end{aligned}
$$

Now let us take

$$
g=\left(\begin{array}{ccc}
I & 0 & 0 \\
G_{21} & I & 0 \\
\bullet & G_{32} & I
\end{array}\right)
$$

and set

$$
G_{21}=\left(\begin{array}{ll}
p & q \\
r & s
\end{array}\right)
$$

Then

$$
G_{32}=J{ }^{\mathrm{t}} G_{21} J=\left(\begin{array}{cc}
p & -r \\
-q & s
\end{array}\right)
$$

and

$$
\tilde{\Omega}_{2}^{2}=\left(\begin{array}{ll}
0 & \sigma \\
\sigma & 0
\end{array}\right)+\left(\begin{array}{cc}
0 & q \omega^{1}+r \omega^{2} \\
q \omega^{1}+r \omega^{2} & 0
\end{array}\right) .
$$

Hence, we can assume that $\tilde{\Omega}_{2}^{2}=0$. In order to keep the form

$$
\Omega_{1}^{2}=\left(\begin{array}{cc}
\omega^{2} & 0 \\
0 & \omega^{1}
\end{array}\right)
$$

i.e. in order to have $\tilde{\omega}_{1}^{4}=\tilde{\omega}_{2}^{3}=0$, it is necessary to assume either: (1) both $G_{11}$ and $G_{22}$ are diagonal; or (2) both $G_{11}$ and $G_{22}$ are anti-diagonal. However, by the second 
condition of (C.2), the case (2) does not occur. Hence we can see that $G_{11}$ is diagonal:

$$
G_{11}=\left(\begin{array}{ll}
a & 0 \\
0 & d
\end{array}\right), \quad G_{22}=I_{2} \text { and } G_{33}=G_{11}^{-1}
$$

accordingly,

$$
\tilde{\Omega}_{1}^{2}=\left(\begin{array}{cc}
a \omega^{2} & 0 \\
0 & d \omega^{1}
\end{array}\right)=\tilde{\Omega}_{2}^{3}
$$

Now insert $\Omega_{2}^{2}=\tilde{\Omega}_{2}^{2}=0$ in the second identity of (C.3) and then we can see that $G_{21}$ must have the form

$$
\left(\begin{array}{ll}
p & 0 \\
0 & s
\end{array}\right)
$$

and

$$
G_{32}=\left(\begin{array}{cc}
p / a & 0 \\
0 & s / d
\end{array}\right) \text {. }
$$

By setting

$$
G_{31}=\left(\begin{array}{ll}
e & f \\
g & h
\end{array}\right)
$$

we check the fourth condition of (C.2). Then we see that $e=p^{2} /(2 d), h=s^{2} /(2 d)$, $g / a=f / d=: \lambda$. Now we have seen that $\tilde{\Omega}_{1}^{1}$ can take the form

$$
\tilde{\Omega}_{1}^{1}=\left(\begin{array}{cc}
\omega_{1}^{1}+d \log a-p \omega^{2} & \frac{a}{d} \omega_{1}^{2} \\
\frac{d}{a} \omega_{2}^{1} & \omega_{2}^{2}+d \log d-s \omega^{1}
\end{array}\right) .
$$

By the fifth formula of (C.3), we can see that

$$
\tilde{\Omega}_{2}^{1}=\left(\begin{array}{cc}
\frac{p}{a} \omega_{1}^{1}+\frac{1}{a}\left(d p+\omega_{3}^{1}\right)-e \omega^{2} & \frac{p}{d} \omega_{1}^{2}+\frac{1}{d} \omega_{3}^{2}-a \lambda \omega^{2} \\
\frac{s}{a} \omega_{2}^{1}+\frac{1}{a} \omega_{4}^{1}-d \lambda \omega^{1} & \frac{s}{d} \omega_{2}^{2}+\frac{1}{d}\left(d s+\omega_{4}^{1}\right)-h \omega^{1}
\end{array}\right) .
$$

It remains to see what $\tilde{\Omega}_{3}^{1}$ is. A computation shows that

$$
\begin{gathered}
\tilde{\Omega}_{3}^{1}=\left(\begin{array}{cc}
0 & \tilde{\tau} \\
\tilde{\tau} & 0
\end{array}\right), \\
\tilde{\tau}=\frac{1}{a d} \tau+d \lambda+\lambda\left(d(\log d)+\omega_{1}^{1}+\omega_{2}^{2}-s \omega^{1}-p \omega^{2}\right) \\
+\frac{p^{2}}{2 a d} \omega_{1}^{2}+\frac{s^{2}}{2 a d} \omega_{2}^{1}+\frac{p}{a d} \omega_{3}^{2}+\frac{s}{a d} \omega_{4}^{1} .
\end{gathered}
$$


Thus we have reduced the form of $\omega$. Next, we examine the integrability. By $d \omega^{1}=$ $\omega_{2}^{2} \wedge \omega^{1}$ and $d \omega^{2}=\omega_{1}^{1} \wedge \omega^{2}$, we can find coordinates $(x, y)$ such that $\omega^{1}=d x$ and $\omega^{2}=d y$. Then $G_{11}$ must be a constant matrix and we may assume that $G_{11}=I_{2}$. In this case, we must have $\omega_{2}^{2} \wedge \omega^{1}=0$ and $\omega_{1}^{1} \wedge \omega^{2}=0$ and, by the formulas $\tilde{\omega}_{1}^{1}=\omega_{1}^{1}-p \omega^{2}$ and $\tilde{\omega}_{2}^{2}=\omega_{2}^{2}-s \omega^{1}$, we can assume that $\omega_{1}^{1}=\omega_{2}^{2}=0$. Then $G_{21}=0$. As we saw $\sigma=0$, it holds that $\omega_{1}^{2} \wedge \omega^{1}=\omega_{2}^{1} \wedge \omega^{2}=0$. This means that we can set $\omega_{1}^{2}=b \omega^{1}$ and $\omega_{2}^{1}=c \omega^{2}$. The first identity of (C.1) becomes $d \omega_{1}^{1}=b c \omega^{1} \wedge \omega^{2}+\omega^{2} \wedge \omega_{3}^{1}$, while $d \omega_{1}^{1}=0$. This implies that there exists a scalar $\kappa$ so that $\omega_{3}^{1}=b c \omega^{1}+\kappa \omega^{2}$. Similarly, from the fourth identity of (C.1), $\omega_{4}^{2}=b c \omega^{2}+\delta \omega^{1}$ for another scalar $\delta$. From the second and the third identities, we may set $\omega_{3}^{2}=b_{y} \omega^{1}+\beta \omega^{2}$ and $\omega_{4}^{1}=c_{x} \omega^{2}+\gamma \omega^{1}$. Furthermore, $\sigma=0$ implies that $\omega_{3}^{2} \wedge \omega^{1}=\omega^{2} \wedge \omega_{4}^{1}$. Hence, $\beta=\gamma$. Here we look at the formula of $\tilde{\Omega}_{2}^{1}$, which says that we can make $\beta=\gamma=0$ by choosing $\lambda$ appropriately. Now we have a final form of $\omega$ that is given in (4.12) by setting $\tau=\mu \omega^{1}+\nu \omega^{2}$. The conditions relative to $d \omega_{3}^{2}$ and $d \omega_{4}^{1}$ show that $\mu=b_{y y}-\kappa b$ and $v=c_{x x}-\delta c$. The conditions relative to $d \omega_{3}^{1}$, $d \omega_{4}^{2}$ and $d \tau$ give the three integrability conditions in (4.14). We thus complete the proof.

Remark C.1. The consideration above works also for the case when the signature is $(4,2)$, which occurs in the Lie sphere geometry of surfaces in Euclidean space. Let $t={ }^{\mathrm{t}}\left(t_{1}, \ldots, t_{6}\right)$ be a frame of $\mathbb{P}^{5}$ endowed with the inner product defined by

$$
(u, v)=-u^{0} v^{0}+u^{1} v^{1}+u^{2} v^{2}+u^{3} v^{3}+u^{4} v^{4}-u^{5} v^{5}
$$

for two vectors $u=\left[u^{0}, \ldots, u^{5}\right]$ and $v=\left[v^{0}, \ldots, v^{5}\right]$. We assume $t$ satisfies the orthonormality $(t, t)=h_{\mathcal{L}}$, where

$$
h_{\mathcal{L}}=\left(\begin{array}{ccc}
0 & 0 & -I_{2} \\
0 & I_{2} & 0 \\
-I_{2} & 0 & 0
\end{array}\right)
$$

Let $Q_{\mathcal{L}}=\left\{u \in \mathbb{P}^{5} ;(u, u)=0\right\}$ be a quadratic hypersurface called the Lie hyperquadric of signature $(4,2)$. We assume that the line $\overline{t_{1} t_{2}}$ lies in $Q_{\mathcal{L}}$ and the conditions (4.15) and (4.16). Then, similarly as in the case of signature $(3,3)$, we can find a transformation $g$ with $g h_{\mathcal{L}}{ }^{\mathrm{t}} g=h_{\mathcal{L}}$ such that the connection form relative to 
the frame $g t$ reduces to

$$
\omega=\left(\begin{array}{cccccc}
0 & b d x & d y & 0 & 0 & 0 \\
c d y & 0 & 0 & d x & 0 & 0 \\
b c d x+\kappa d y & b_{y} d x & 0 & 0 & d y & 0 \\
c_{x} d y & b c d y+\delta d x & 0 & 0 & 0 & d x \\
0 & \mu d x+v d y & b c d x+\kappa d y & c_{x} d y & 0 & -c d y \\
-\mu d x-v d y & 0 & b_{y} d x & b c d y+\delta d x & -b d x & 0
\end{array}\right)
$$

and, in this case, we have identities

$$
\mu=b_{y y}-b \kappa, \quad v=-c_{x x}+c \delta
$$

and the integrability condition is

$$
\kappa_{x}=(b c)_{y}+c b_{y}, \quad \delta_{y}=(b c)_{x}+b c_{x}, \quad v_{x}-\mu_{y}=-\delta c_{x}-\kappa b_{y} .
$$

The formulas (C.4)-(C.6) are different from the formulas (4.12)-(4.14) in Section 4.8 only in \pm -signs of certain variables. It is in $[\mathbf{F e 0 0 b}, \mathbf{F e 0 2}]$ that the dual correspondence is shown to be possible. The case where the system (4.12) describes the image of a projectively minimal surface corresponds to the case where the system (C.4) describes a Lie minimal surface. A unified approach to both minimal surfaces was given in [BHJ02].

\section{REFERENCES}

[AG93] M. A. Akivis and V. V. Goldberg. Projective Differential Geometry of Submanifolds. NorthHolland, Amsterdam, 1993.

[Bol] G. Bol. Projektive Differentialgeometrie, Vol. 1. Vandenhoeck and Rüprecht, Göttingen, 1950. G. Bol. Projektive Differentialgeometrie, Vol. 2. Vandenhoeck and Rüprecht, Göttingen, 1954 G. Bol. Projektive Differentialgeometrie, Vol. 3. Vandenhoeck and Rüprecht, Göttingen, 1967.

[BHJ02] F. Burstall and U. Hertrich-Jeromin. Harmonic maps in unfashionable geometries. Manuscripta Math. 108 (2002), 171-189.

[BS99] A. I. Bobenko and W. K. Schief. Discrete indefinite affine spheres. Discrete Integrable Geometry and Physics (Vienna, 1996) (Oxford Lecture Series in Mathematics and its Applications, 16). Oxford University Press, New York, 1999.

[Ca20] E. Cartan. Sur la deformation projective des surfaces. Ann. École Norm. 37 (1920), 259-356.

[Ca37] E. Cartan. Leçons sur la théorie des espaces à connexion projective. Gauthier-Villars, Paris, 1937.

[Ch36] S. S. Chern. Associate quadratic complexes of a rectilinear congruence. Tôhoku Math. J. 40 (1936), 293-316. 
[Ch44] S. S. Chern. Laplace transforms of a class of higher dimensional varieties in a projective space of $n$ dimensions. Proc. Nat. Acad. Sci. 30 (1944), 95-97.

[Ch47] S. S. Chern. Sur une classe remarquable de variété dans l'espace projectif à $n$ dimensions. Science Reports Nat. Tsing Hua Univ. 4 (1947), 328-336.

[D1] G. Darboux. Leçons sur la théorie générale des surfaces, Théorie génerale des surfaces, Vol. I, 1887.

[D2] G. Darboux. Leçons sur la théorie générale des surfaces, Théorie génerale des surfaces, Vol. II, 1889.

[Dem11] A. Demoulin. Sur les surfaces $R$ et les surfaces $\Omega$. Comptes Rendus 153 (1911), 590-593, 705-705.

[Dem20] A. Demoulin. Sur la transformation de Moutard et quelques-unes de ses applications géométriques. Bull. Classe des Sci., Acad. Royale de Belg. 5 (1919), 262-284.

A. Demoulin. Sur les léquations de Moutard à solutions quadratiques, Bull. Acad. Belgique (5) 6 (1920), 192-215, 418-458, 503-523.

A. Demoulin. Sur les léquations de Moutard à solutions quadratiques, Bull. Acad. Belgique (5) 7 (1921), 10-32.

[Dem24] A. Demoulin. Sur les surfaces dont les quadriques de Lie n'ont que deux points caractéristique. Comptes Rendus 179 (1924), 20-22.

[E09] L. P. Eisenhart. A Treatise on the Differential Geometry of Curves and Surfaces. Ginn \& Co, Boston, MA, 1909.

[Fe00a] E. V. Ferapontov. Integrable systems in projective differential geometry. Kyushu J. Math. 54 (2000), 183-215.

[Fe00b] E. V. Ferapontov. Lie sphere geometry and integrable systems. Tôhoku Math. J. 52 (2000), 199-233.

[Fe02] E. V. Ferapontov. Analog of Wilczynski's projective frame in Lie sphere geometry: Lieapplicable surfaces and commuting Schrödinger operators with magnetic fields. Intern. J. Math. 13 (2002), 959-985.

[FS99] E. V. Ferapontov and W. K. Schief. Surfaces of Demoulin: differential geometry, Bäcklund transformation and integrability. J. Geom. Phys. 30 (1999), 343-363.

[Fi30] S. Finikoff. Sur les quadriques de Lie et les congruences de Demoulin. Mat. Sbornik 37 (1930), 48-97.

[Fi32] S. Finikoff. Congruences dont les deux nappes de la surface focale sont projectivement applcables l'une sur l'autre par les points correspondantes. Bull. Sci. Math. (2) 56 (1932), $117-136$.

[Fi59] S. Finikow. Theorie der Kongruenzen. Akademie-Verlag, Berlin 1959.

[FC1] G. Fubini and E. Čeck. Geometria proiettiva differenziale, Vol. I. Zanichelli, Bologna, 1926.

G. Fubini and E. Ceck. Geometria proiettiva differenziale, Vol. II. Zanichelli, Bologna, 1927. G. Fubini and E. Čeck. Introduction à la géométrie projective différentielle des surfaces. Gauthier-Villars, Paris, 1931.

[G34] L. Godeaux. La thérie des surfaces et l'espace de reglé. Hermann, Paris, 1934.

[Ha83] G. H. Halphen. Sur les invariants des équations différentiells linéaires du quatrième ordre. Acta Math. 3 (1883), 325-380.

[Hu93] H. S. Hu. Darboux transformations of Su-chain. 'Differential Geometry', Proceedings of the Symposium in honour of Professor Su Buchin on his 90th Birthday. Eds. C. H. Gu, H. S. Hu and Y. L. Xin. World Scientific, Singapore, 1993, 108-113.

[KT96] N. Kamran and K. Tenenblat. Laplace transformation in higher dimensions. Duke Math. J. 84 (1996), 237-266. 
[Ka22] J. Kanitani. On the projective deformation of curved surfaces, I, II, III. Memoirs of Coll. Science, Kyoto Imperial Univ. 6 (1922), 1-43 and 77-96.

[Ka33] J. Kanitani. Projective Differential Geometry. Iwanami, Tokyo, 1933 (in Japanese).

[Ku60] E. E. Kummer. Allgemeine Theorie der geradlinigen Strahlensysteme. J. reine angew. Math. 57 (1860), 189-230.

[L42] E. P. Lane. A Treatise on Projective Differential Geometry. University of Chicago Press, Chicago, IL, 1942.

[Mar79] F. Marcus. On the $\mathcal{D}$-correspondence of coincidence surfaces. Acad. Roy. Belg. Bull. Cl. Sci. (5) 65 (1979), 407-424

[Mar80] F. Marcus. On a result of Tzitzeica and a new asymptotic transformation of minimal projective surfaces. Czech. Math. J. 30 (1980), 213-227.

[May32] O. Mayer. Contribution à l'étude des surfaces minima projectives. Bull. Sci. Math. (2) 56 (1932), 146-168, 188-200.

[MSY93] K. Matsumoto, T. Sasaki, and M. Yoshida. Recent progress of Gauss-Schwarz theory and related geometric structures. Mem. Fac. Sci, Kyushu Univ., Ser. A, Mathematics, 47 (1993), 283-381.

[NS94] K. Nomizu and T. Sasaki. Affine Differential Geometry, Geometry of Affine Immersions (Cambridge Tracts in Mathematics, 111). Cambridge University Press, Cambridge, 1994.

[O88] K. Okamoto. Échelles et l'équation de Toda, Équations différentielles dans le champ complexe, Vol. II. Publ. Inst. Rech. Math. Av., Univ. Louis Pasteur, 1988, pp. 19-38.

[RS02] C. Rogers and W. K. Schief. Bäcklund and Darboux Transformations (Cambridge Texts in Applied Mathematics). Cambridge University Press, Cambridge, 2002.

[Sa87] T. Sasaki. On a projectively minimal hypersurface in the unimodular affine space. Geom. Dedicata 23 (1987), 237-251.

[Sa88] T. Sasaki. On the projective geometry of hypersurfaces, Équations différentielles dans le champ complexe, Vol. III, Publ. Inst. Rech. Math. Av., Univ. Louis Pasteur, 1988, 115-161.

[Sa91] T. Sasaki. Contiguity relations of Aomoto-Gel'fand hypergeometric functions and applications to Appell's system $F_{3}$ and Goursat's system ${ }_{3} F_{2}$. SIAM J. Math. Anal. 22 (1991), 821-846.

[Sa99] T. Sasaki. Projective Differential Geometry and Linear Homogeneous Differential Equations (Rokko Lectures in Mathematics, 5). Department of Mathematics, Kobe University, 1999.

[Sa01] T. Sasaki. Projective surfaces defined by Appell's hypergeometric systems $E_{4}$ and $E_{2}$. Kyushu J. Math. 55 (2001), 329-350.

[Se87] Y. Se-ashi. A geometric construction of Laguerre-Forsyth's canonical forms of linear ordinary differential equations (Preprint, 1987). Adv. Stud. Pure Math. 22 (1993), 265-297.

[Se88] Y. Se-ashi. On differential invariants of integrable finite type linear differential equations. Hokkaido Math. J. 17 (1988), 151-195.

[Su35] Su Buchin. On the surfaces whose asymptotic curves belong to linear complexes, I. Tôhoku Math. J. 40 (1935), 408-420.

Su Buchin. On the surfaces whose asymptotic curves belong to linear complexes, II. Tôhoku Math. J. 40 (1935), 433-448.

[Su36a] Su Buchin. On certain periodic sequence of Laplace of period four in ordinary space. Sci. Reports of Tôhoku Imp. Univ. 25 (1936), 227-256.

[Su36b] Su Buchin. Some characteristic properties of projective minimal surfaces. Rep. Tôhoku Univ. 24 (1936), 595-600.

[Su57] Su Buchin. On Demoulin transforms of projective minimal surfaces (I). Acta Math. Sinica 7 (1957), 28-50.

[Su64] Su Buchin. Introduction to projective surfaces. Shanghai Scientific and Technical Publishers, Shanghai, (in Chinese), 1964. 
[SYY97] T. Sasaki, K. Yamaguchi, and M. Yoshida. On the rigidity of differential systems modelled on Hermitian symmetric spaces and disproofs of a conjecture concerning modular interpretations of configuration spaces. Adv. Stud. Pure Math. 25 (1997), 318-354.

[Th26] G. Thomsen. Sulle superficie minime proiettive. Ann. Mat. Pura Appl. 5 (1928), 169-184.

[Tz07] G. Tzitzeica. Sur une nouvelle classe de surfaces. C. R. Acad. Sci. Paris 144 (1907), 12571259.

G. Tzitzeica. Sur une nouvelle classe de surfaces. C. R. Acad. Sci. Paris 150 (1910), 955-956.

[Tz24] G. Tzitzeica. Géométrie Différentielle projectives des Réseaux. Gauthier-Villars, Paris, 1924.

[W06] E. J. Wilczynski. Projective Differential Geometry of Curves and Ruled Surfaces. Teubner, Stuttgart, 1906.

[W07] E. J. Wilczynski. Projective differential geometry of curved surfaces (first memoir). Trans. Amer. Math. Soc. 8 (1907), 233-260.

[W11] E.J. Wilczynski, Sur la théorie genérale des congruences. Mém. Publ. Classe Sci. Acad. R. Belgique (2) 3 (1911), 1-86.

[W15] E. J. Wilczynski. The general theory of congruences. Trans. Amer. Math. Soc. 16 (1915), 311327.

Takeshi Sasaki

Department of Mathematics

Kobe University

Rokko

Kobe 657-8501

Japan

(E-mail: sasaki@math.kobe-u.ac.jp) 